Substituição de valores ausentes: uma abordagem baseada em um algoritmo evolutivo para agrupamento de dados 



\section{Substituição de valores ausentes: uma abordagem baseada em um algoritmo evolutivo para agrupamento de dados ${ }^{1}$}

Jonathan de Andrade Silva

Orientador: Prof. Dr. Eduardo Raul Hruschka

Dissertação apresentada ao Instituto de Ciências Matemáticas e de Computação - ICMC-USP, como parte dos requisitos necessários à obtenção do título de Mestre em Ciências da Computação e Matemática Computacional.

\section{USP - São Carlos}

\section{Abril/2010}

\footnotetext{
${ }^{1}$ Trabalho Realizado com Suporte Financeiro da CNPq, Processo n ${ }^{\circ}$ 132113/2009-0, e CAPES
} 

À minha família 



\section{Agradecimentos}

A Deus por me iluminar, dar-me forças e conduzir-me pelo caminho reto.

Aos meus pais, Zilto e Maria, e minhas irmãs, Jennifer e Jéssica, pelo incentivo e carinho ao longo de minha vida.

Ao meu orientador, prof. Eduardo Raul Hruschka, por participar ativamente na minha formação acadêmica, pela orientação, apoio e amizade.

Aos amigos de trabalho, futebol e alguns churrascos, em especial Wesley Nunes, Bruno Brandoli, Pâmela Nunes, Diogo Nunes, Thiago Ferreira, Lucas Vendramin, Ricardo Cerri, Pablo Andretta, Murilo Naldi, Luiz Colleta, Danilo Horta, Débora Medeiros e Márcio Basgalupp.

A todos os funcionários do ICMC, em especial a Ana Paula pela eficiência, apoio e sua dedicação com o trabalho.

Ao CNPq e a CAPES pelo auxílio financeiro. 



\section{Resumo}

A substituição de valores ausentes, também conhecida como imputação, é uma importante tarefa para a preparação dos dados em aplicações de mineração de dados. Este trabalho propõe e avalia um algoritmo para substituição de valores ausentes baseado em um algoritmo evolutivo para agrupamento de dados. Este algoritmo baseia-se na suposição de que grupos (previamente desconhecidos) de dados podem prover informações úteis para o processo de imputação. Para avaliar experimentalmente o algoritmo proposto, simulações de valores ausentes foram realizadas em seis bases de dados, para problemas de classificação, com a aplicação de dois mecanismos amplamente usados em experimentos controlados: MCAR e MAR. Os algoritmos de imputação têm sido tradicionalmente avaliados por algumas medidas de capacidade de predição. Entretanto, essas tradicionais medidas de avaliação não estimam a influência dos métodos de imputação na etapa final em tarefas de modelagem (e.g., em classificação). Este trabalho descreve resultados experimentais obtidos sob a perspectiva de predição e inserção de tendências (viés) em problemas de classificação. Os resultados de diferentes cenários nos quais o algoritmo proposto, apresenta em geral, desempenho semelhante a outros seis algoritmos de imputação reportados na literatura. Finalmente, as análises estatísticas reportadas sugerem que melhores resultados de predição não implicam necessariamente em menor viés na classificação. 



\section{Abstract}

The substitution of missing values, also called imputation, is an important data preparation task for data mining applications. This work proposes and evaluates an algorithm for missing values imputation that is based on an evolutionary algorithm for clustering. This algorithm is based on the assumption that clusters of (partially unknown) data can provide useful information for the imputation process. In order to experimentally assess the proposed method, simulations of missing values were performed on six classification datasets, with two missingness mechanisms widely used in practice: MCAR and MAR. Imputation algorithms have been traditionally assessed by some measures of prediction capability. However, this traditionall approach does not allow inferring the influence of imputed values in the ultimate modeling tasks (e.g., in classification). This work describes the experimental results obtained from the prediction and insertion bias perspectives in classification problems. The results illustrate different scenarios in which the proposed algorithm performs similarly to other six imputation algorithms reported in the literature. Finally, statistical analyses suggest that best prediction results do not necessarily imply in less classification bias. 

Esta dissertação foi preparada com o formatador de textos $\mathrm{LT}_{\mathrm{E}} \mathrm{X}$. O estilo utilizado no documento foi desenvolvido por Ronaldo Cristiano Prati. A bibliografia foi gerada automaticamente pelo BIBT $\mathrm{E}$, utilizando o estilo Chicago com modificações para o português.

Algumas palavras utilizadas neste trabalho não foram traduzidas da língua inglesa para a portuguesa por serem amplamente conhecidas e difundidas na comunidade acadêmica. 



\section{Sumário}

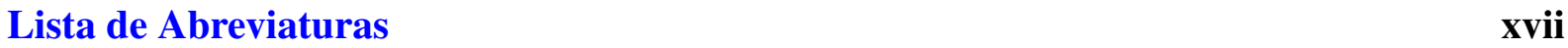

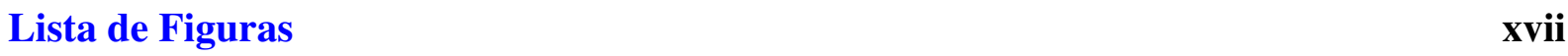

Lista de Tabelas $\quad$ xxi

Lista de Algoritmos $\quad$ xxix

1 Introdução 1

1.1 Contextualização e Motivação . . . . . . . . . . . . . . . . . . 1

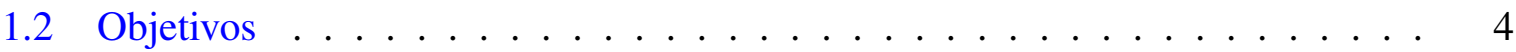

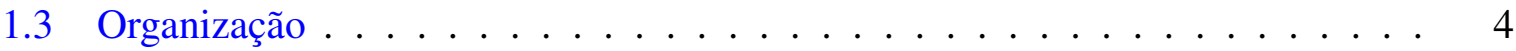

2 Substituição de Valores Ausentes 5

2.1 Valores Ausentes . . . . . . . . . . . . . . . . . . . 5 5

2.2 Métodos Tradicionais para a Substituição de Valores Ausentes . . . . . . . . . 7

2.3 Imputação Baseada em Vizinhos Mais Próximos . . . . . . . . . . . . . 8

2.3.1 Algoritmo KNNimpute (Troyanskaya et al., 2001) . . . . . . . . . . 9

2.3.2 Algoritmo SKNN (Kim et al., 2004) . . . . . . . . . . . . . . . 11

2.3.3 Algoritmo KNN Iterativo (Brás e Menezes, 2007) . . . . . . . . . . . . 11

2.3.4 Algoritmo KMI (Hruschka et al., 2004) . . . . . . . . . . . . . . . . . 12

2.3.5 Considerações sobre os Algoritmos de Imputação Baseados em Vizinhos Mais Próximos . . . . . . . . . . . . . . . . . . . . . 14

3 EACImpute: Algoritmo Evolutivo para Imputação Baseado em Agrupamento 17

3.1 Considerações Iniciais ～. . . . . . . . . . . . . . . . . . . . 17

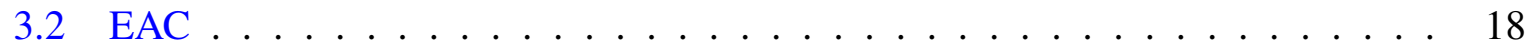

3.2.1 Esquema de Codificação . . . . . . . . . . . . . . . . . . . . . 19

3.2 .2 Processo de Busca Local . . . . . . . . . . . . . . . . . . . . . . 19 
3.2.3 Função de Adequabilidade . . . . . . . . . . . . . . . . . . . 20

3.2 .4 Seleção . . . . . . . . . . . . . . . . . . . 20

3.2 .5 Operadores de Mutação . . . . . . . . . . . . . . . . . 21

3.3 EACImpute . . . . . . . . . . . . . . . . . 23

3.3 .1 Imputação . . . . . . . . . . . . . . . . . . 24

4 Resultados Experimentais $\quad 27$

4.1 Bases de Dados . . . . . . . . . . . . . . . . . . . . . . . . 27

4.2 Simulação de Valores Ausentes . . . . . . . . . . . . . . . . . . . 28

4.2.1 Mecanismo de Ausência MCAR - Completamente Aleatório . . . . . . 28

4.2.2 Mecanismo de Ausência MAR - Aleatório . . . . . . . . . . . . . . . . 28

4.3 Metodologia . . . . . . . . . . . . . . . . . . 34

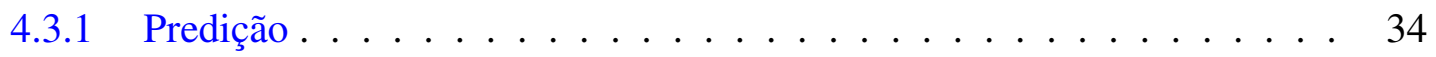

4.3 .2 Classificação . . . . . . . . . . . . . . . . . . . 34

4.4 Configuração de Parâmetros para os Algoritmos de Imputação . . . . . . . . . 39

4.5 Resultados Obtidos . . . . . . . . . . . . . . . . . . . . . 40

4.5.1 Completamente Aleatório - MCAR . . . . . . . . . . . . . 41

4.5 .2 Aleatório - MAR . . . . . . . . . . . . . . . . 65

4.5.3 Considerações Finais . . . . . . . . . . . . . . . . . . . 82

5 Conclusão e Trabalhos Futuros $\quad 93$

5.1 Conclusões . . . . . . . . . . . . . . . . . . . . . . . 93

5.2 Contribuições . . . . . . . . . . . . . . . . . . . . . . . . 94

5.3 Publicações Geradas . . . . . . . . . . . . . . . . . . . . . 94

5.4 Trabalhos Futuros . . . . . . . . . . . . . . . . . . . . 95

$\begin{array}{ll}\text { Referências Bibliográficas } & 97\end{array}$

$\begin{array}{lll}\text { A Apêndice } & 103\end{array}$

A.1 Completamente Aleatório - MCAR . . . . . . . . . . . . . . . . . . 103

A.1.1 Base de dados "Íris" . . . . . . . . . . . . . . . . . . . 103

A.1.2 Base de Dados "Glass Identification" . . . . . . . . . . . . . . . . 106

A.1.3 Base de Dados "Yeast" . . . . . . . . . . . . . . . . . . . . . . . 108

A.1.4 Base de Dados "Pen-Digts" . . . . . . . . . . . . . . . . . . . 110

A.1.5 Base de Dados "Segmentation" . . . . . . . . . . . . . . . . . . 112

A.2 Aleatório - MAR . . . . . . . . . . . . . . . . . . . . 114

A.2.1 Base de Dados "Sintética" . . . . . . . . . . . . . . . . . . . . . 114

A.2.2 Base de Dados "Íris" . . . . . . . . . . . . . . . . . . . . . 116

A.2.3 Base de Dados "Segmentation" . . . . . . . . . . . . . . . . . 119 


\section{Lista de Figuras}

2.1 Separação da matriz de dados $X_{D}$ em $X_{C}$ (instâncias completas) e $X_{A}$ (instâncias ausentes). A matriz $X_{A}$ está ordenada pela taxa de ausentes. . . . . 12

3.1 Representação gráfica dos dados: eixos representam atributos e símbolos representam grupos. . . . . . . . . . . . . . . . . . . . . . . . . . 19

3.2 Representação genotípica da partição apresentada na Figura 3.1. O elemento de índice 13 contém o número de grupos codificados. . . . . . . . . . . . . . . 19

3.3 Aplicação do operador de mutação para remoção de grupos. . . . . . . . . . . 22

3.4 Aplicação do operador de mutação para divisão de grupos. . . . . . . . . . . . 23

3.5 Processo ilustrativo da imputação executada no EACImpute. . . . . . . . . . . 26

$4.1 \quad$ Base de dados sintética. . . . . . . . . . . . . . . . . . . . . . . . . . . . 29

4.2 Base de dados sintética com valores ausentes simulados por meio do mecanismo MAR com probabilidade $p(y=? \mid x \in[0,2])=0,5$. O quadrado ilustra os pontos (instâncias) que conterão valores ausentes. . . . . . . . . . . . . . 31

4.3 Base de dados Íris, visualizada por meio dos 2 melhores atributos (LP e CP). . 32

4.4 Simulação de valores ausentes no mecanismo MAR para a base de dados Íris. Na Figura (a),(b) e (c) são apresentadas respectivamente exemplos para a primeira, segunda e a terceira forma de simulação de valores ausentes. Cada quadrado, envolvendo os símbolos “ $\times$ ” e “•”, indica que a instância contém valores ausentes no atributo LP. . . . . . . . . . . . . . . . . . . . . . . 33

4.5 Exemplo ilustrativo do processo de imputação de maneira supervisionada. . . . 35

4.6 Exemplo ilustrativo do viés de classificação. . . . . . . . . . . . . . . . . . 37

4.7 Procedimento para estimar o viés na etapa de classificação. . . . . . . . . . . 38

4.8 Avaliação pelo $\overline{E Q_{N}}$ para a base de dados Íris. Na Figura (a) são apresentados os resultados de todos algoritmos. Na Figura (b) são apresentados os resultados do erro desconsiderando o algoritmo Média Geral (pior algoritmo). . . . . . . . 
4.9 Avaliação por meio da estimação do viés na classificação. Na Figura (a) são apresentados os resultados dos algoritmos comparados com o algoritmo Média Geral. Na Figura (b) são apresentados os resultados dos valores médios do viés, desconsiderando o algoritmo Média Geral. . . . . . . . . . . . . . . 45

4.10 Avaliação pelo $\overline{E Q_{N}}$ para a taxa de ausentes em 10\%, 30\%, 50\% e 70\% . . . .

4.11 Avaliação pela estimação do viés na classificação para a taxa de ausentes em $10 \%, 30 \%, 50 \%$ e $70 \%$. A Figura (a) apresenta os resultados dos algoritmos comparados com o algoritmo Média geral. A Figura (b) apresenta os resultados do viés na classificação desconsiderando o algoritmo Média geral. . . . . . . .

4.12 Avaliação pelo $\overline{E Q_{N}}$ para a taxa de ausentes em $10 \%, 30 \%, 50 \%$ e $70 \%$. . . .

4.13 Resultados médios da avaliação pela estimação do viés na classificação para a taxa de ausentes em 10\%, 30\%, 50\% e 70\% . . . . . . . . . 53

4.14 Avaliação pelo $\overline{E Q_{N}}$ para a taxa de ausentes em $10 \%, 30 \%, 50 \%$ e $70 \%$. . . . 56

4.15 Avaliação por meio da estimação do viés na classificação para a taxa de ausentes em $10 \%, 30 \%, 50 \%$ e $70 \% \ldots \ldots \ldots \ldots$

4.16 Avaliação pelo $\overline{E Q_{N}}$ para a taxa de ausentes em $10 \%, 30 \%, 50 \%$ e $70 \%$. . . . 61

4.17 Avaliação pelo bias de classificação para a taxa de ausentes em 10\%, 30\%, 50\%

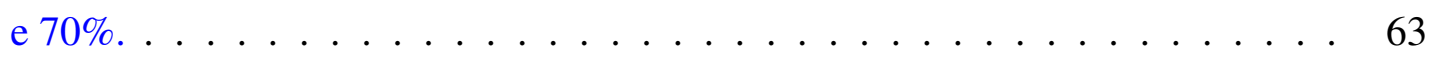

4.18 Resultado da imputação dos valores ausentes para o algoritmo Média Majority para a taxa de $30 \%$ de valores ausentes. . . . . . . . . . . . .

4.19 Resultado da imputação dos valores ausentes para os algoritmos de imputação (Média Majority, KNNImpute, SKNN, IKNNImpute e EACImpute) na taxa de $30 \%$ de valores ausentes. . . . . . . . . . . . . . . . 68

4.20 Avaliação pelo $\overline{E Q_{N}}$ para a taxa de ausentes em $10 \%, 30 \%, 50 \%$ e $70 \%$. . . . 69

4.21 Avaliação por meio da estimação do viés na classificação para a base Sintética. 70

4.22 Fronteira de decisão (esquerda) construída pelo algoritmo J4.8 (direita) para os valores estimados com o algoritmo Média Majority na taxa de $30 \%$ de valores ausentes. . . . . . . . . . . . . . . . . . .

4.23 Resultados para $\overline{E Q_{N}}$, base de dados Íris-1, para cada cenário de ausência simulados com probabilidades $p(L P=? \mid C P \in[3,7])=p_{i}$, sendo $p_{i} \in$ $\{0.1,0.3,0.5,0.7\} \ldots \ldots \ldots \ldots \ldots$

4.24 Resultados para a estimação do viés de classificação, base de dados Íris-1, para cada taxa de valores ausentes simulados com probabilidade $p(L P=? \mid 3 \leq$ $C P \leq 7)=p_{i}$, na qual $p_{i} \in\{0.1,0.3,0.5,0.7\}$ e $i=\{1, \ldots, 4\} \ldots \ldots$

4.25 Resultados para $\overline{E Q_{N}}$, base de dados Íris-2 para cada taxa de valores ausentes simulados com probabilidades $p(L P=? \mid 4.5 \leq C P \leq 5.1)=p_{i}$, sendo $p_{i} \in$ $\{0.1,0.3,0.5,0.7\}$ e $i=\{1, \ldots, 4\} \ldots \ldots \ldots$ 
4.26 Resultados referentes à estimação do viés na classificação, base de dados Íris2, para cada taxa de valores ausentes simulados com probabilidades $p(L P=$ $? \mid 4.5 \leq C P \leq 5.1)=p_{i}$, sendo $p_{i} \in\{0.1,0.3,0.5,0.7\} \ldots \ldots \ldots$

4.27 Resultados para $\overline{E Q_{N}}$, base de dados Íris-3, para cada cenário de ausência com valores ausentes simulados com probabilidades $p(L P=$ ? $\mid 3.5 \leq C P \leq$ $4.5 \vee 5.1 \leq C P \leq 7])=p_{i}$, sendo $p_{i} \in\{0.1,0.3,0.5,0.7\} \ldots \ldots \ldots$

4.28 Resultados para a estimação do viés de classificação, base de dados Íris-3, para cada taxa de valores ausentes simulados com probabilidades $p(L P=? \mid 3.5 \leq$ $C P \leq 4.5 \vee 5.1 \leq C P \leq 7])=p_{i}$, sendo $p_{i} \in\{0.1,0.3,0.5,0.7\} \ldots \ldots 79$

4.29 Avaliação pelo $\overline{E Q_{N}}$ para a base de dados Segmentation. . . . . . . . . . . 79

4.30 Avaliação para o viés de classificação para a base de dados Segmentation. . . . 81

4.31 Resultados gerais para o mecanismo MCAR. . . . . . . . . . . . . . 85

4.32 Correlação entre os resultados do $\overline{E Q_{N}}$ e viés de classificação para o mecanismo MCAR . . . . . . . . . . . . . . . . . . . 86

4.33 Resultados gerais para o mecanismo MAR. . . . . . . . . . . . . . 87

4.34 Correlação entre os resultados do $\overline{E Q_{N}}$ e viés de classificação para o mecanismo MAR. . . . . . . . . . . . . . . . . . . . . . . 89 



\section{Lista de Tabelas}

2.1 Questionário sobre renda de casais. . . . . . . . . . . . . . 6

2.2 Exemplo de valores ausentes distribuídos de forma "completamente aleatória" (MCAR). ......................... 6

2.3 Exemplo de valores ausentes distribuídos de forma "aleatória" (MAR). . . . . . 7

2.4 Exemplo de valores ausentes distribuídos de forma "não aleatória" (NMAR). 7

4.1 Principais características das bases de dados utilizadas. . . . . . . . . . . . . . 28

4.2 Resultados de acurácia para os classificadores J4.8, $k$-NN, MLP e NB. Em negrito estão destacados as melhores taxas de classificação corretas médias (TCCM) para cada base de dados. . . . . . . . . . . . . . . . . . .

4.3 "Ranks" médios em cada cenário de ausência $-\overline{E Q_{N}}$ para a base de dados Íris. Os menores valores de "rank" médios estão destacados em negrito. . . . . . . . . 43

4.4 Total de vitórias, empates e derrotas, em relação ao "rank" médio $-\overline{E Q_{N}}$ para a base de dados Íris. . . . . . . . . . . . . . . . . . . . . . . . 43

4.5 Total de vitórias, empates e derrotas de cada algoritmo obtido dos cenários de ausência em que houve diferenças estatísticas $-\overline{E Q_{N}}$ para a base de dados Íris.

4.6 "Ranks" médios em cada cenário de ausência - viés de classificação para a base de dados Íris. Os menores valores de "rank" médios estão destacados em negrito. 44

4.7 Total de vitórias, empates e derrotas, em relação ao "rank" médio - viés de classificação para a base de dados Íris. . . . . . . . . . . . . . . . . . . 45

4.8 Total de vitórias, empates e derrotas em relação às diferenças estatísticas para cada cenário de ausência - viés de classificação para a base de dados Íris. . . . .

4.9 "Ranks" médios em cada cenário de ausência $-\overline{E Q_{N}}$ para a base de dados Glass Identification. Os menores valores de "rank" médios estão destacados em negrito. . . . . . . . . . . . . . . . . .

4.10 Total de vitórias, empates e derrotas em relação ao "rank" médio em cada cenário de ausência $-\overline{E Q_{N}}$ para a base de dados Glass Identification. . . . . . 
4.11 Total de vitórias, empates e derrotas de cada algoritmo que houve diferença estatística na comparação pareada em cada cenário de ausência $-\overline{E Q_{N}}$ para a base de dados Glass Identification. . . . . . . . . . . . . . . . . . . . .

4.12 "Ranks" médios em cada cenário de ausência - estimação do viés da classificação para a base de dados Glass Identification. Os menores valores de "rank" médios estão destacados em negrito.

4.13 Total de vitórias, empates e derrotas em relação ao "rank" médio em cada cenário de ausência - estimação do viés de classificação para a base de dados Glass Identification.

4.14 Total de vitórias, empates e derrotas em relação às diferenças estatísticas na comparação pareada em cada cenário de ausência - estimação do viés na classificação para a base de dados Glass Identification. . . . . . . . . . . . . .

4.15 "Ranks" médios em cada cenário de ausência $-\overline{E Q_{N}}$ para a base de dados Yeast. Os menores valores de "rank" médios estão destacados em negrito. . . . .

4.16 Total de vitórias, empates e derrotas, em relação aos "ranks" médios relativos ao $\overline{E Q_{N}}$ para a base de dados Yeast. . . . . . . . . . . . . . . . . . . .

4.17 Total de vitórias, empates e derrotas de cada algoritmo que houve diferença estatística na comparação pareada em cada cenário de ausência $-\overline{E Q_{N}}$ para a base de dados Yeast. . . . . . . . . . . . . . . . . . . . . . . . . . .

4.18 "Ranks" médios em cada cenário de ausência - estimação do viés da classificação para a base de dados Yeast. Os menores valores de "rank" médios estão destacados em negrito. . . . . . . . . . . . . . . . . . . . . . .

4.19 Total de vitórias, empates e derrotas, em relação aos "ranks" médios relativos à estimação do viés na classificação para a base de dados Yeast. . . . . . . . . . .

4.20 Total de vitórias, empates e derrotas de cada algoritmo que houve diferença estatística na comparação pareada em cada cenário de ausência - estimação do viés na classificação para a base de dados Yeast. . . . . . . . . . . . . . . . .

4.21 "Ranks" médios em cada cenário de ausência $-\overline{E Q_{N}}$ para a base de dados PenDigits. Os menores valores de "rank" médios estão destacados em negrito. . . .

4.22 Total de vitórias, empates e derrotas, em relação aos "ranks" médios relativos ao $\overline{E Q_{N}}$ para a base de dados Pen-Digits. . . . . . . . . . . . . . . . . .

4.23 Total de vitórias, empates e derrotas de cada algoritmo que houve diferença estatística na comparação pareada em cada cenário de ausência $-\overline{E Q_{N}}$ para a base de dados Pen-Digits. . . . . . . . . . . . . . . . . . .

4.24 "Ranks" médios em cada cenário de ausência - estimação do viés na classificação para a base de dados Pen-Digits. Os menores valores de "rank" médios estão destacados em negrito. . . . . . . . . . . . . . . .

4.25 Total de vitórias, empates e derrotas, em relação aos "ranks" médios relativos à estimação do viés na classificação para a base de dados Pen-Digits. 
4.26 Total de vitórias, empates e derrotas de cada algoritmo que houve diferença estatística na comparação pareada em cada cenário de ausência - estimação do viés na classificação para a base de dados Pen-Digits. . . . . . . . . . . . . . .

4.27 "Ranks" médios em cada cenário de ausência $-\overline{E Q_{N}}$ para a base de dados Segmentation. Os menores valores de "rank" médios estão destacados em negrito.

4.28 Total de vitórias, empates e derrotas, em relação aos "ranks" médios $-\overline{E Q_{N}}$ para a base de dados Segmentation. . . . . . . . . . . . . . . .

4.29 Total de vitórias, empates e derrotas por meio das comparações pareadas de cada algoritmo que houve diferenças estatísticas em cada cenário de ausência $-\overline{E Q_{N}}$ para a base de dados Segmentation. . . . . . . . . . . . . . . .

4.30 "Ranks" médios em cada cenário de ausência - estimação do viés na classificação para a base de dados Segmentation. Os menores valores de "rank" médios estão destacados em negrito. . . . . . . . . . . . . . . . . .

4.31 Total de vitórias, empates e derrotas, em relação aos "ranks" médio - estimação do viés na classificação para a base de dados Segmentation. . . . . . . . . . . .

4.32 Total de vitórias, empates e derrotas por meio das comparações pareadas entre os algoritmos quando houve diferença estatística em cada cenário de ausência - estimação do viés na classificação para a base de dados Segmentation. . . .

4.33 "Ranks" médios em cada cenário de ausência $-\overline{E Q_{N}}$ para a base de dados Sintética. Os menores valores de "rank" médios estão destacados em negrito. .

4.34 Total de vitórias, empates e derrotas, em relação aos "ranks" médios $-\overline{E Q_{N}}$ para a base de dados Sintética . . . . . . . . . . . . . . . .

4.35 Total de vitórias, empates e derrotas, em relação às diferenças estatísticas, de cada algoritmo $-\overline{E Q_{N}}$ para a base de dados Sintética. . . . . . . . . . . .

4.36 "Ranks" médios em cada cenário de ausência - estimação do viés de classificação para a base de dados Sintética. Os menores valores de "rank" médios estão destacados em negrito. . . . . . . . . . . . . . . . . . . 70

4.37 Total de vitórias, empates e derrotas, em relação aos "ranks" médios estimação do viés na classificação para a base de dados Sintética . . . . . . . . .

4.38 Total de vitórias, empates e derrotas, em relação às diferenças estatísticas para a avaliação - estimação do viés na classificação para a base de dados Sintética.

4.39 "Ranks" médios em cada taxa de ausentes $-\overline{E Q_{N}}$ para a base de dados Íris-1. Os menores valores de "rank" médios estão destacados em negrito. . . . . . . . .

4.40 Total de vitórias, empates e derrotas, em relação aos "ranks" médios $-\overline{E Q_{N}}$ para a base de dados Íris-1. . . . . . . . . . . . . . . . . . . .

4.41 Total de vitórias, empates e derrotas, em relação às diferenças estatísticas, para cada cenário de ausência $-\overline{E Q_{N}}$ para a base de dados Íris-1. . . . . . . . . . . . 
4.42 "Ranks" médios em cada taxa de ausentes - estimação do viés de classificação para a base de dados Íris-1. Os menores valores de "rank" médios estão destacados em negrito.

4.43 "Ranks" médios para cada taxa de ausentes $-\overline{E Q_{N}}$ para a base de dados Íris-2. Os menores valores de "rank" médios estão destacados em negrito. . . . . . . . .

4.44 Total de vitórias, empates e derrotas, em relação ao "rank" médio, avaliados por meio do $\overline{E Q_{N}}$ para a base de dados Íris-2 . . . . . . . . . . . . . . . . . 75

4.45 Total de vitórias, empates e derrotas, de cada algoritmo que houve diferença estatística na comparação pareada em cada cenário de ausência $-\overline{E Q_{N}}$ para a base de dados Íris-2.

4.46 "Ranks" médios em cada taxa de ausentes - estimação do viés na classificação para a base de dados Íris-2. Os menores valores de "rank" médios estão destacados em negrito.

4.47 "Ranks" médios em cada taxa de ausentes $-\overline{E Q_{N}}$ para a base de dados Íris-3. Os menores valores de "rank" médios estão destacados em negrito. . . . . . . . .

4.48 Total de vitórias, empates e derrotas, em relação ao "rank" médio $-\overline{E Q_{N}}$ para a base de dados Íris-3. . . . . . . . . . . . . . . . . . . . . .

4.49 Total de vitórias, empates e derrotas, em relação às diferenças estatísticas $-\overline{E Q_{N}}$ para a base de dados Íris-3.

4.50 "Ranks" médios em cada taxa de ausentes - estimação do viés na classificação para a base de dados Íris-3. Os menores valores de "rank" médios estão destacados em negrito. . . . . . . . . . . . . . . . . . .

4.51 "Ranks" médios em cada taxa de ausentes $-\overline{E Q_{N}}$ para a base de dados Segmentation. Os menores valores de "rank" médios estão destacados em negrito.

4.52 Total de vitórias, empates e derrotas, em relação ao "rank" médio $-\overline{E Q_{N}}$ para a base de dados Segmentation. . . . . . . . . . . . . . . . . . . . . . 80

4.53 Total de vitórias, empates e derrotas, em relação às diferenças estatísticas $-\overline{E Q_{N}}$ para a base de dados Segmentation. . . . . . . . . . . . . .

4.54 "Ranks" médios em cada taxa de ausentes - estimação do viés de classificação para a base de dados Segmentation. Os menores valores de "rank" médios estão destacados em negrito.

4.55 Total de vitórias, empates e derrotas, em relação ao "rank" médio - viés de classificação para a base de dados Segmentation. . . . . . . . . . . . . .

4.56 Total de vitórias, empates e derrotas, em relação às diferenças estatísticas viés de classificação para a base de dados Segmentation.

4.57 Resultados gerais para a base de dados Íris com valores ausentes simulados pelo mecanismo MCAR.

4.58 Resultados gerais para a base de dados Glass Identification com valores ausentes simulados pelo mecanismo MCAR. . . . . . . . . . . . . . 
4.59 Resultados gerais para a base de dados Yeast com valores ausentes simulados pelo mecanismo MCAR. . . . . . . . . . . . . . . . . . . . . 84

4.60 Resultados gerais para a base de dados Pen-Digits com valores ausentes simulados pelo mecanismo MCAR. . . . . . . . . . . . . . . . . . . . 84

4.61 Resultados gerais para a base de dados Segmentation com valores ausentes simulados pelo mecanismo MCAR. . . . . . . . . . . . . . . . . .

4.62 Resultados gerais para a base de dados Sintética com valores ausentes simulados pelo mecanismo MAR . . . . . . . . . . . . . . . . . . . . . . . . 88

4.63 Resultados gerais para a base de dados Íris-1 com valores ausentes simulados pelo mecanismo MAR. . . . . . . . . . . . . . . . . . . 88

4.64 Resultados gerais para a base de dados Íris-2 com valores ausentes simulados pelo mecanismo MAR. . . . . . . . . . . . . . . . . . . . . . .

4.65 Resultados gerais para a base de dados Íris-3 com valores ausentes simulados pelo mecanismo MAR.

4.66 Resultados gerais para a base de dados Segmentation com valores ausentes simulados pelo mecanismo MAR.

A.1 Resultados da comparação pareada (Vitórias/Empates/derrotas) dos "ranks" médios em cada taxa de ausentes $-\overline{E Q_{N}}$ para base de dados Íris.

A.2 Resultados da comparação pareada (Vitórias/Empates/derrotas), em relação às diferenças estatísticas em cada taxa de ausentes $-\overline{E Q_{N}}$ para base de dados Íris. 104

A.3 Resultados da comparação pareada (Vitórias/Empates/derrotas), dos "ranks" médios em cada taxa de ausentes - viés na classificação para base de dados Íris.

A.4 Resultados da comparação pareada (Vitórias/Empates/derrotas), em relação às diferenças estatísticas em cada taxa de ausentes - viés na classificação para base de dados Íris.

A.5 Resultados da comparação pareada (Vitórias/Empates/derrotas), dos "ranks" médios em cada taxa de ausentes - viés para base de dados Glass Identification. 106

A.6 Resultados da comparação pareada (Vitórias/Empates/derrotas), em relação às diferenças estatísticas em cada taxa de ausentes $-\overline{E Q_{N}}$ para a base de dados Glass Identification.

A.7 Resultados da comparação pareada (Vitórias/Empates/derrotas), dos "ranks" médios em cada taxa de ausentes - viés na classificação para a base de dados Glass Identification.

A.8 Resultados da comparação pareada (Vitórias/Empates/derrotas), em relação às diferenças estatísticas observadas em cada taxa de ausentes - viés na classificação para a base de dados Glass Identification. 
A.9 Resultados da comparação pareada (Vitórias/Empates/derrotas) dos "ranks" médios em cada taxa de ausentes $-\overline{E Q_{N}}$ para a base de dados Yeast. . . . . . . 108

A.10 Resultados da comparação pareada (Vitórias/Empates/derrotas) em relação às diferenças estatísticas observadas em cada taxa de ausentes $-\overline{E Q_{N}}$ para a base de dados Yeast. . . . . . . . . . . . . . . . . . . . . . . 108

A.11 Resultados da comparação pareada (Vitórias/Empates/derrotas), dos "ranks" médios em cada taxa de ausentes - viés na classificação para a base de dados Yeast. . . . . . . . . . . . . . . . . . . . . . . . 109

A.12 Resultados da comparação pareada (Vitórias/Empates/derrotas) em relação às diferenças estatísticas observadas em cada taxa de ausentes - viés na classificação para a base de dados Yeast.

A.13 Resultados da comparação pareada (Vitórias/Empates/derrotas) em relação às diferenças estatísticas observadas em cada taxa de ausentes $-\overline{E Q_{N}}$ para a base de dados Pen-Digits.

A.14 Resultados da comparação pareada (Vitórias/Empates/derrotas) em relação às diferenças estatísticas observadas em cada taxa de ausentes $-\overline{E Q_{N}}$ para a base de dados Pen-Digits. . . . . . . . . . . . . . . . . . .

A.15 Resultados da comparação pareada (Vitórias/Empates/derrotas) em relação aos valores médios de "ranks" em cada taxa de ausentes - viés na classificação para a base de dados Pen-Digits. . . . . . . . . . . . . . . . . . . . . . 111

A.16 Resultados da comparação pareada (Vitórias/Empates/derrotas) em relação às diferenças estatísticas observadas em cada taxa de ausentes - viés na classificação para a base de dados Pen-Digits.

A.17 Resultados da comparação pareada (Vitórias/Empates/derrotas) dos "ranks" médios em cada taxa de ausentes $-\overline{E Q_{N}}$ para a base de dados Segmentation.

A.18 Resultados da comparação pareada (Vitórias/Empates/derrotas) em relação às diferenças estatísticas observadas em cada taxa de ausentes $-\overline{E Q_{N}}$ para a base de dados Segmentation. . . . . . . . . . . . . . . . . . . . . .

A.19 Resultados da comparação pareada (Vitórias/Empates/derrotas) dos "ranks" médios em cada taxa de ausentes - viés na classificação para a base de dados Segmentation.

A.20 Resultados da comparação pareada (Vitórias/Empates/derrotas) em relação às diferenças estatísticas observadas em cada taxa de ausentes - viés na classificação para a base de dados Segmentation. . . . . . . . . . . . . .

A.21 Resultados da comparação pareada (Vitórias/Empates/derrotas) dos "ranks" médios em cada taxa de ausentes $-\overline{E Q_{N}}$ para a base de dados Sintética. . . . 114

A.22 Resultados da comparação pareada (Vitórias/Empates/derrotas) em relação às diferenças estatísticas observadas em cada taxa de ausentes $-\overline{E Q_{N}}$ para a base de dados Sintética. 
A.23 Resultados da comparação pareada (Vitórias/Empates/derrotas) dos "ranks" médios em cada taxa de ausentes - viés na classificação para a base de dados Sintética.

A.24 Resultados da comparação pareada (Vitórias/Empates/derrotas) em relação às diferenças estatísticas observadas em cada taxa de ausentes - viés na classificação para a base de dados Sintética.

A.25 Resultados da comparação pareada (Vitórias/Empates/derrotas) dos "ranks" médios em cada taxa de ausentes $-\overline{E Q_{N}}$ para a base de dados Íris-1 . . . . . . 116

A.26 Resultados da comparação pareada (Vitórias/Empates/derrotas) em relação às diferenças estatísticas observadas em cada taxa de ausentes $-\overline{E Q_{N}}$ para a base de dados Íris-1. . . . . . . . . . . . . . . . . . . . . . . . . . 116

A.27 Resultados da comparação pareada (Vitórias/Empates/derrotas) dos "ranks" médios em cada taxa de ausentes $-\overline{E Q_{N}}$ para a base de dados Íris-2.

A.28 Resultados da comparação pareada (Vitórias/Empates/derrotas) em relação às diferenças estatísticas observadas em cada taxa de ausentes $-\overline{E Q_{N}}$ para a base de dados Íris-2. . . . . . . . . . . . . . . . . . . . . . . . . . . . 11

A.29 Resultados da comparação pareada (Vitórias/Empates/derrotas) dos "ranks" médios em cada taxa de ausentes $-\overline{E Q_{N}}$ para a base de dados Íris-3. . . . . . 118

A.30 Resultados da comparação pareada (Vitórias/Empates/derrotas) em relação as diferenças estatísticas observadas em cada taxa de ausentes $-\overline{E Q_{N}}$ para a base de dados Íris-3. . . . . . . . . . . . . . . . . . . . . . . . . . . . . . 118

A.31 Resultados da comparação pareada (Vitórias/Empates/derrotas) dos "ranks" médios em cada taxa de ausentes $-\overline{E Q_{N}}$ para a base de dados Segmentation.

A.32 Resultados da comparação pareada (Vitórias/Empates/derrotas) em relação às diferenças estatísticas em cada taxa de ausentes $-\overline{E Q_{N}}$ para a base de dados Segmentation.

A.33 Resultados da comparação pareada (Vitórias/Empates/derrotas) em relação às diferenças estatísticas observadas em cada taxa de ausentes - viés na classificação para a base de dados Segmentation. 



\section{Lista de Algoritmos}

1 Algoritmo de imputação KNNimpute . . . . . . . . . . . . . . . . . . . . 10

2 Algoritmo de imputação $S K N N \ldots \ldots \ldots$. . . . . . . . . . . . . . 11

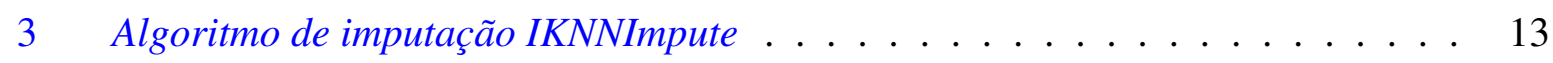

$4 \quad$ Algoritmo de imputação KMI . . . . . . . . . . . . . . . . . . . . . . . . 13

5 Algoritmo Evolutivo de Agrupamento EAC . . . . . . . . . . . . . . . . . . 18

6 Algoritmo para remoção de grupos (Operador de Mutação 1) . . . . . . . . . . 21

7 Algoritmo para divisão de grupos (Operador de Mutação 2) . . . . . . . . . . . 22

8 Algoritmo de Imputação EACImpute . . . . . . . . . . . . . . . . . . . . . . . . 24

9 Algoritmo de Simulação MCAR . . . . . . . . . . . . . . . . . . . . . 29 



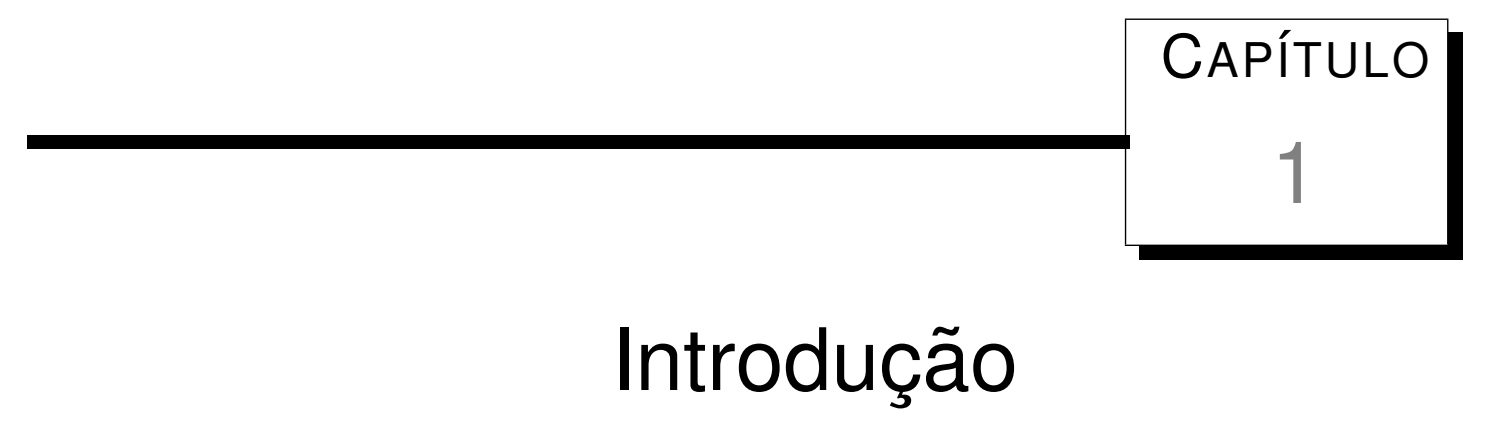

\subsection{Contextualização e Motivação}

Os avanços recentes em hardware e software têm proporcionado a aquisição de dados em grande escala. Devido às limitações humanas em lidar com grandes quantidades de dados, soluções automatizadas necessitam ser aplicadas para eficientemente e efetivamente extrair informação e conhecimento desses dados. Essa necessidade é objeto de estudo do campo de pesquisa conhecido como Mineração de Dados, do inglês Data Mining (Fayyad et al., 1996), o qual está relacionado ao desenvolvimento de ferramentas para a exploração do dados em busca padrões válidos e potencialmente úteis. Para minerar dados, algoritmos de aprendizagem de máquina são tipicamente usados para a descoberta de conhecimento. Aprendizagem de máquina envolve o estudo de métodos computacionais que aprendem e aperfeiçoam o seu desempenho com a experiência (Mitchell, 1997). Métodos de aprendizagem de máquina têm sido utilizados em diversas aplicações, como por exemplo, detecção de fraudes em transações bancárias, desenvolvimento de jogos e análise de registros médicos.

Uma grande preocupação para a comunidade de aprendizagem de máquina e outras áreas relacionadas à descoberta de conhecimento envolve a qualidade dos dados. Isto porque a eficácia dos resultados obtidos por métodos baseados nesses conceitos são dependentes da qualidade dos dados (Cios et al., 2007). Pesquisas relacionadas à qualidade dos dados envolvem a avaliação dos dados e o desenvolvimento de métodos para tratar dados de qualidade ruim. A avaliação dos dados está relacionada à combinação de uma avaliação subjetiva e objetiva (Pipino et al., 2002). Métodos para limpeza dos dados (data cleaning), por sua vez, são rotineiramente aplicados para preencher dados incompletos (ausência de valores em alguns atributos), suavizar dados ruidosos, identificar ou remover outliers e corrigir inconsistências nos dados (Han e Kamber, 2000). 
Uma importante tarefa para os métodos destinados à limpeza de dados consiste em tratar dados com valores ausentes (Cios et al., 2007). Dados com valores ausentes são comumente encontrados em aplicações reais e podem ocorrer por diversas razões, tais como: omissão de dados pela própria fonte de informação (e.g., salário numa pesquisa de opinião) e mau-funcionamento do dispositivo de coleta de dados (e.g., manômetros ou pluviômetros).

Embora alguns algoritmos de aprendizagem de máquina sejam tolerantes aos valores ausentes (Breiman et al., 1984; Brazdil e Bruha, 1992; Quinlan, 1993), a sua maioria não apresenta esta capacidade (Pyle, 1999; Johansson e Hakkinen, 2006). Neste sentido, vários métodos têm sido propostos para lidar com valores ausentes (Pyle, 1999; Schafer, 2000). Um método simples para lidar com valores ausentes consiste em ignorar instâncias ${ }^{1}$ e/ou atributos contendo valores ausentes. Porém, o desperdício de dados pode ser considerável, podendo influenciar negativamente nas análises dos dados. Em Kim et al. (2004), foi verificado que após remover dados de expressão gênica com valores ausentes de um conjunto contendo 6361 genes (instâncias) e 156 medidas (atributos), para a realização de seus experimentos, houve uma redução de $88 \%$ dos dados. Dessa maneira, torna-se necessário abordar novas estratégias para tratar o problema. Uma estratégia envolve a substituição dos valores ausentes. O processo de substituição de valores ausentes, também conhecido como imputação, visa o preenchimento dos valores ausentes por valores mais prováveis. Esse processo consiste na estimação de valores por meio das informações disponíveis no conjunto de dados. Essa estratégia é mais flexível, de modo que o método adotado para substituição de valores ausentes pode ser aplicado independente do algoritmo de aprendizagem de máquina utilizado, proporcionado ao usuário escolher o método mais adequado para o caso em estudo.

Algumas técnicas para substituição dos valores ausentes envolvem a estimação de parâmetros baseada no bem conhecido algoritmo de maximização da esperança ExpectationMaximization (EM) (Dempster et al., 1977; Redner e Walker, 1984; Ghahramani e Jordan, 1994; Jordan e Xu, 1995; Bilmes, 1997). Este algoritmo é baseado em uma função de probabilidade, que preenche os valores ausentes por meio de uma distribuição que modela os dados. Os parâmetros dessa distribuição são inicialmente definidos pelo usuário. Dentre os algoritmos baseados em aprendizagem de máquina, as árvores de decisão apresentam resultados práticos em lidar com instâncias contendo valores ausentes, conforme se pode encontrar em (Lakshminarayan et al., 1996; Lobo e Numao, 2000; Conversano e Cappelli, 2002). Porém, os algoritmos de imputação baseados em árvores de decisão não lidam naturalmente com atributos contendo valores contínuos e necessitam induzir uma árvore para cada atributo com valores ausentes. Outro conceito consiste em imputações múltiplas (Rubin, 1977, 1987). Imputações múltiplas têm sido amplamente utilizadas para análise multivariada, usando mais do que um valor para preencher os valores ausentes em uma amostra (e.g., a média dos prováveis valores), mas envolvem um custo computacional consideravelmente alto (Cartwright et al., 2003).

\footnotetext{
${ }^{1} \mathrm{O}$ termo instância pode ser também compreendido como um objeto, uma tupla ou um registro de um conjunto de dados.
} 
O método dos vizinhos mais próximos - $k$-Nearest Neighbours $(k-\mathrm{NN})$ - tem sido amplamente utilizado para o propósito de imputação (Troyanskaya et al., 2001; Jonsson e Wohlin, 2004; Brás e Menezes, 2007). Em particular, o método conhecido por KNNimpute (Troyanskaya et al., 2001), é bastante utilizado para a imputação. Este método de imputação encontra-se disponível em sistemas amplamente usados na prática, tais como: SAM ${ }^{2}$ (Tusher et al., 2001), PAM $^{3}$ (Tibshirani et al., 2002) e MAANOVA ${ }^{4}$ (Kerr et al., 2000) que realizam análises estatísticas de expressões gênicas (microarray) (Scheel et al., 2005). Métodos baseados no $k$-NN têm apresentado resultados promissores. Motivado pela ampla aplicação do método dos vizinhos mais próximos e sua eficácia para a imputação, este trabalho está focado em métodos de imputação baseados no $k$-NN. No contexto de imputação, métodos baseados no $k$-NN procuram pelas instâncias da base de dados mais similares à instância que possui valores ausentes. A instância mais similar - segundo alguma medida de similaridade - é chamada de vizinha mais próxima. Assim, podem-se obter $k$ vizinhos mais próximos, usando-os para substituir os valores ausentes (Troyanskaya et al., 2001; Batista e Monard, 2003; Kim et al., 2004; Hruschka et al., 2004). No entanto, este método necessita da definição a priori do número de vizinhos $(k)$. Uma solução investigada, implementada e avaliada nesse trabalho envolve a aplicação de uma abordagem baseada em agrupamento de dados com intuito de fornecer um conjunto de vizinhos mais prováveis como entrada para o $k$-NN na estimação dos valores ausentes. Em particular, neste trabalho, é abordada uma técnica de agrupamento que envolve a obtenção de partições rígidas, na qual cada instância pertence apenas a um grupo.

O processo de agrupamento de dados envolve a identificação de um conjunto de categorias - também chamadas de grupos ou de clusters $^{5}$ - que descrevam um conjunto de dados (Fayyad et al., 1996), objetivando-se maximizar a homogeneidade entre os objetos de um mesmo grupo e, concomitantemente, maximizar a heterogeneidade entre objetos de grupos distintos. Algoritmos para agrupamento de dados são capazes de explorar relações presentes entre pares de atributo-valor de uma base de dados e, portanto, os grupos obtidos podem indiretamente refletir tais relações. Em particular, grupos de dados podem ser vistos como informações granulares (Pedrycz, 2005) que sumarizam a distribuição espacial dos dados. Tais informações granulares podem fornecer uma estimativa viável para preencher os valores ausentes com mínima distorção dos valores que estão atualmente presentes no conjunto de dados. Dessa maneira, vários algoritmos para agrupamento de dados podem ser adaptados para a proposta de imputação. Por exemplo, o popular algoritmo das $K$-médias poderia ser um candidato devido a sua escalabilidade e simplicidade de implementação. No entanto, este algoritmo apresenta duas limitações bem conhecidas: sensibilidade à inicialização dos protótipos e a definição a priori do número de grupos. Para superar essas limitações foi utilizado o algoritmo de agrupamento de dados

\footnotetext{
${ }^{2}$ www-stat.stanford.edu/tibs/SAM

${ }^{3}$ www-stat.stanford.edu/tibs/PAM

${ }^{4}$ http://research.jax.org/faculty/churchill/software/Rmaanova

${ }^{5}$ Denomina-se aqui de cluster um grupo de dados, enquanto que um agrupamento (ou alternativamente, uma partição) é um conjunto de clusters.
} 
evolutivo EAC (Hruschka et al., 2006). Porém, este algoritmo não lida naturalmente com valores ausentes, sendo então necessário adaptá-lo para o propósito de imputação. Este processo de adaptação é objeto de estudo nesse trabalho.

\subsection{Objetivos}

Uma preocupação emergente em tarefas de modelagem envolve avaliar o impacto dos métodos de imputação em tarefas de modelagem. Dessa maneira, este trabalho se concentra fundamentalmente no desenvolvimento e na avaliação de um algoritmo para imputação em problemas de classificação. Trata-se, portanto, de um trabalho focado na tarefa de preparação de dados.

Resumidamente, os objetivos específicos desse trabalho foram:

- Desenvolvimento de um algoritmo, baseado no conceito de agrupamento de dados, para o pré-processamento de bases de dados com valores ausentes. De maneira sucinta, grupos contendo instâncias com valores ausentes terão tais valores (códigos de ausência) substituídos por valores conhecidos das instâncias pertencentes ao mesmo grupo. O método proposto foi desenvolvido com o objetivo de preencher duas lacunas. A primeira está relacionada à definição do número de vizinhos - para o método dos vizinhos mais próximos, enquanto que a segunda está relacionada ao o número de grupos - para o método baseado no agrupamento de dados.

- Avaliação do impacto dos valores imputados em problemas de classificação. De maneira específica, pretende-se avaliar o grau de distorções das características do conjunto de dados, as quais se refletem no relacionamento entre os atributos. Neste sentido, em Hruschka et al. (2009b) foi proposta uma metodologia para estimar o nível de tendências inseridas pelo processo de imputação em problemas de classificação. Esta metodologia de avaliação foi utilizada neste trabalho.

\subsection{Organização}

No Capítulo 2 são apresentados conceitos básicos relativos aos problemas com valores ausentes, bem como os métodos de imputação baseados nos vizinhos mais próximos implementados neste trabalho. No Capítulo 3 é descrito o algoritmo proposto. Resultados experimentais obtidos pela comparação entre o método proposto e alguns métodos importantes baseados nos vizinhos mais próximos descritos na literatura são apresentados no Capítulo 4. Finalmente, no Capítulo 5 são apresentadas as considerações finais, os principais resultados e algumas sugestões para trabalhos futuros. 


\section{$-$ \\ Substituição de Valores Ausentes}

Neste capítulo são apresentados conceitos relativos à taxonomia dos valores ausentes e os métodos tradicionais para lidar com tais valores. Além disso, métodos de imputação comumente utilizados na literatura e que foram estudados e implementados para a realização de experimentos neste trabalho são também apresentados.

\subsection{Valores Ausentes}

Um dos princípios básicos para a utilização de um procedimento de imputação consiste em determinar o que vem a ser um valor ausente (Schafer e Graham, 2002). Um valor ausente é usualmente representado por um código de ausência - por exemplo, pode ser um símbolo, um valor específico ou um espaço em branco, caracterizando que um determinado valor não foi observado ou simplesmente foi ignorado. A ocorrência de tais valores normalmente torna difícil a tarefa de análise de dados. Quando tal ocorrência foge do nosso controle é possível fazer suposições sobre o modo de como estes valores estão distribuídos (Schafer e Graham, 2002). Segundo a tipologia de Rubin (Little e Rubin, 2002) existem três formas de caracterizar o modo pelo qual os valores ausentes estão distribuídos:

- Completamente aleatório (Missing Completely At Random - MCAR).

- Aleatório (Missing At Random - MAR).

- Não aleatório (Not Missing At Random - NMAR).

Os valores ausentes estão distribuídos na forma MCAR quando a probabilidade de uma instância ter um valor ausente não depende de nenhum valor da base de dados. Em relação ao modo MAR, a probabilidade de uma instância possuir um valor ausente depende dos valores 
conhecidos na base de dados. Por fim, os valores ausentes são classificados como não aleatório (NMAR) quando a probabilidade de uma instância conter valores ausentes depende dos próprios valores ausentes. Para exemplificar os conceitos relacionados à categorização dos valores ausentes (MCAR, MAR e NMAR), considere um exemplo no qual é aplicado um questionário para obter informações sobre a renda familiar de casais, conforme apresentado na Tabela 2.1. Neste exemplo, três atributos (Casal, Renda-Homem e Renda-Mulher) são apresentados. Considere que desses três atributos apenas os atributos Renda-Homem e Renda-Mulher podem conter valores ausentes, representados pelo símbolo “?”. Na Tabela 2.2 é apresentada uma matriz de dados contendo valores ausentes de forma completamente aleatória (MCAR). Neste caso, os códigos de ausência foram inseridos de maneira completamente aleatória na base de dados. No tipo de ausência MAR, a probabilidade de um atributo (por exemplo, Renda-Homem) conter valores ausentes depende dos valores observados nos outros atributos (neste caso, RendaMulher). Na Tabela 2.3, os casais 2 e 6 não possuem os valores para o atributo Renda-Homem. Isso ocorre quando algum valor do atributo Renda-Mulher é superior ou igual a $\mathrm{R} \$ 5.500,00$. Neste exemplo, todos os casais em que o valor do atributo Renda-Mulher é superior ou igual a R \$ 5.500,00 apresentam altas probabilidades de conter valores ausentes. De maneira formal, pode-se escrever, por exemplo $p$ (Renda-Homem $=$ ? $\mid$ Renda-Mulher $\geq 5.500,00)=0,95$. Por fim, no modo de ausência não aleatória (NMAR) a probabilidade de uma instância conter valores ausentes para um atributo (e.g., Renda-Homem) depende do próprio valor do atributo (neste caso, Renda-Homem), conforme o exemplo apresentado na Tabela 2.4, na qual os casais 4 e 5 contêm valores ausentes quando o valor de Renda-Homem é superior ou igual a $R \$$ $5.300,00$. Neste caso, homens cujas rendas são maiores ou iguais a $\mathrm{R} \$ 5.300,00$ provavelmente não informarão suas respectivas rendas.

\begin{tabular}{|c|c|c|}
\hline Casal & Renda-Homem $(\mathrm{R} \$)$ & Renda-Mulher(R\$) \\
\hline 1 & $1.200,00$ & $1.200,00$ \\
\hline 2 & $3.250,00$ & $6.500,00$ \\
\hline 3 & $3.750,00$ & $1.870,00$ \\
\hline 4 & $6.200,00$ & $3.450,00$ \\
\hline 5 & $5.300,00$ & $2.750,00$ \\
\hline 6 & $3.750,00$ & $5.500,00$ \\
\hline
\end{tabular}

Tabela 2.1: Questionário sobre renda de casais.

\begin{tabular}{|c|c|c|}
\hline Casal & Renda-Homem (R\$) & Renda-Mulher(R\$) \\
\hline 1 & $?$ & $1.200,00$ \\
\hline 2 & $3.250,00$ & $6.500,00$ \\
\hline 3 & $3.750,00$ & $?$ \\
\hline 4 & $?$ & $3.450,00$ \\
\hline 5 & $5.300,00$ & $2.750,00$ \\
\hline 6 & $?$ & $5.500,00$ \\
\hline
\end{tabular}

Tabela 2.2: Exemplo de valores ausentes distribuídos de forma "completamente aleatória" (MCAR). 


\begin{tabular}{|c|c|c|}
\hline Casal & Renda-Homem $(\mathrm{R} \$)$ & Renda-Mulher(R\$) \\
\hline 1 & $1.200,00$ & $1.200,00$ \\
\hline 2 & $?$ & $6.500,00$ \\
\hline 3 & $3.750,00$ & $1.870,00$ \\
\hline 4 & $6.200,00$ & $3.450,00$ \\
\hline 5 & $5.300,00$ & $2.750,00$ \\
\hline 6 & $?$ & $5.500,00$ \\
\hline
\end{tabular}

Tabela 2.3: Exemplo de valores ausentes distribuídos de forma "aleatória” (MAR).

\begin{tabular}{|c|c|c|}
\hline Casal & Renda-Homem $(\mathrm{R} \$)$ & Renda-Mulher(R\$) \\
\hline 1 & $\mathrm{R} \$: 1.200,00$ & $\mathrm{R} \$: 1.200,00$ \\
\hline 2 & $\mathrm{R} \$: 3.250,00$ & $\mathrm{R} \$: 6.500,00$ \\
\hline 3 & $\mathrm{R} \$: 3.750,00$ & $\mathrm{R} \$: 1.870,00$ \\
\hline 4 & $?$ & $\mathrm{R} \$: 3.450,00$ \\
\hline 5 & $?$ & $\mathrm{R} \$: 2.750,00$ \\
\hline 6 & $\mathrm{R} \$: 3.750,00$ & $\mathrm{R} \$: 5.500,00$ \\
\hline
\end{tabular}

Tabela 2.4: Exemplo de valores ausentes distribuídos de forma "não aleatória” (NMAR).

O modo de ausência NMAR não é comumente utilizado na literatura para simulação de valores ausentes em experimentos controlados (Zhang et al., 2005; Nguyen et al., 2004). Isto porque na prática de imputação é difícil encontrar uma suposição NMAR (Qin et al., 2009), pois os métodos de imputação utilizam a informação disponível dos dados para estimar os valores ausentes. Considerando o exemplo da Tabela 2.4, para uma melhor compreensão, todo casal em que o valor do atributo Renda-Homem é superior ou igual a $\mathrm{R} \$ 5.300,00$, conforme pode ser verificado na Tabela 2.1, terá uma probabilidade elevada de conter valores ausentes no atributo Renda-Homem. Assim, os algoritmos de imputação usualmente não encontrarão informações suficientes sobre os casais em que a renda do homem é superior ou igual a $R \$ 5.300,00$ para estimar os valores ausentes desses casais.

\subsection{Métodos Tradicionais para a Substituição de Valores Au- sentes}

A substituição de valores ausentes, também conhecida como imputação, consiste em estimar tais valores baseando-se nas informações disponíveis no conjunto de dados. Métodos para lidar com valores ausentes têm sido estudados por muitos anos (Anderson, 1946; Preece, 1971; Dempster et al., 1977; Rubin, 1977, 1987; Schafer, 2000). Muitos desses métodos têm sido aplicados para análise de dados de entrevistas, usualmente não sendo úteis para aplicações de mineração de dados. Os principais métodos de imputação podem ser caracterizados como (Batista, 2003):

- Imputação pela média ou moda. Esta técnica de imputação consiste em substituir os valores ausentes de cada atributo pela média (para atributos numéricos) ou pela moda (para atributos nominais) dos valores conhecidos do conjunto de dados. Este método faz com 
que a variância populacional seja subestimada. Em problemas de classificação, uma alternativa consiste em utilizar a informação da classe do conjunto de dados para imputar os valores ausentes. Este método é conhecido como método Majority (Kononenko et al., 1984) e consiste em aplicar o processo de imputação de maneira estratificada, de forma que, para cada classe, um atributo que contém valores ausentes tem seus valores imputados pela média (ou moda) dos seus valores conhecidos.

- Imputação local (procedimentos Hot-deck ou Cold-deck). Para cada instância com valores ausentes é encontrado um subconjunto de instâncias completas mais similares e os valores ausentes são preenchidos por meio deste subconjunto. O procedimento Hot-deck geralmente inicia-se com a divisão das instâncias de um conjunto de dados em grupos ("clusters"). Em seguida, cada instância desse conjunto de dados com valores ausentes é associada a um grupo mais próximo. As instâncias completas pertencentes a um grupo são então utilizadas para estimar os valores ausentes das instâncias do mesmo grupo. Usualmente a média ou a moda dos valores do atributo das instâncias completas é utilizada. O procedimento Cold-deck difere do Hot-deck apenas no processo de agrupamento que é realizado em um conjunto de dados distinto daquele que contém valores ausentes a serem preenchidos.

- Modelos preditivos. Esta técnica engloba métodos estatísticos e probabilísticos para a obtenção de um modelo preditivo para preencher os valores ausentes. O atributo com valores ausentes do conjunto de dados é utilizado como o atributo classe, e o restante dos atributos são utilizados como entrada para a criação do modelo de predição. Em seguida, o modelo preditivo, podendo ser de classificação ou de regressão, irá predizer os valores ausentes. Este processo geralmente é realizado para cada atributo com valores ausentes. Segundo Batista (2003), neste método é necessário assumir que há correlação entre os atributos para que o modelo possa predizer os valores ausentes de maneira apurada. Além disso, os valores preditos pelo modelo são bem mais comportados do que os valores reais.

\subsection{Imputação Baseada em Vizinhos Mais Próximos}

Os algoritmos de imputação baseados no $k$-NN se enquadram no conjunto de métodos que fundamentam-se no procedimento Hot-deck (Jonsson e Wohlin, 2004). Dessa forma, um conjunto de instâncias mais similares à instância com valores ausentes é utilizado para o propósito de imputação. Estes algoritmos podem lidar com atributos numéricos e nominais, e não necessitam construir um modelo para cada atributo com valores ausentes. Além disso, tais algoritmos facilmente podem lidar com instâncias contendo vários valores ausentes e podem levar em consideração a estrutura de correlação dos dados (Acuna e Rodriguez, 2004). Estas características colaboram para tornar tais algoritmos amplamente usados na prática (Troyanskaya et al., 2001; Batista, 2003; Jonsson e Wohlin, 2004; Kim et al., 2004; Brás e Menezes, 2007). 


\subsubsection{Algoritmo KNNimpute (Troyanskaya et al., 2001)}

Nesta seção é descrito o algoritmo de imputação proposto por Troyanskaya et al. (2001), denominado KNNimpute. O algoritmo KNNimpute seleciona as instâncias que são mais similares a uma instância de interesse - para a qual deseja-se preencher valores ausentes. De maneira mais específica, considere uma base de dados $X_{D}$ contendo um conjunto de $N$ instâncias $\left(X_{D}=\left\{\mathbf{x}^{1}, \mathbf{x}^{2}, \ldots, \mathbf{x}^{N}\right\}\right)$, no qual cada instância de $X_{D}$ é representada por um vetor $\mathbf{x}^{i}=\left[x_{1}^{i}, x_{2}^{i}, \ldots, x_{m}^{i}\right]$, em que $m$ é o número de atributos da base de dados. Se uma determinada instância $\mathbf{x}^{i} \in X_{D}$ contém um atributo $a_{l}(l=1, \ldots, m)$ com valor ausente, aqui representado pelo símbolo "?" - como por exemplo, $\mathbf{x}^{i}=\left[?, x_{2}^{i}, \ldots, x_{m}^{i}\right]$ - o método encontra $k$ instâncias similares que não têm valores ausentes no atributo $x_{1}^{i}$, sendo $k$ um valor definido pelo usuário. Uma matriz $R$ é utilizada para mapear os valores ausentes de $X_{D}$. A matriz $R$ contém um conjunto de vetores, correspondentes aos vetores da matriz $X_{D}$, definidos por $R=\left\{\mathbf{r}^{1}, \ldots, \mathbf{r}^{i}, \ldots, \mathbf{r}^{N}\right\}$, com $N$ elementos. Neste caso, se o valor de $x_{l}^{i}$ está ausente, então o valor de $r_{l}^{i}$ é igual a zero; caso contrário é igual a 1.

No algoritmo KNNimpute as instâncias são representadas por pontos no espaço Euclidiano de dimensão $m$. Assim, os vizinhos mais próximos a uma instância podem ser considerados aqueles que apresentam menores distâncias. O cálculo da distância entre as instâncias é realizado por meio da Equação (2.1). Considere-se por exemplo, o cálculo da distância entre as instâncias $\mathrm{x}^{i}$ e $\mathrm{x}^{j}$, para $\mathrm{x}^{i} \neq \mathrm{x}^{j}$, sendo que a instância $\mathrm{x}^{j}$, dada por $\mathbf{x}^{j}=\left[x_{1}^{j}, x_{2}^{j}, \ldots, x_{l-1}^{j}, ?, x_{l+1}^{j}, \ldots, x_{m}^{j}\right]$, contém o $l$-ésimo atributo com valor ausente. Usandose a Equação (2.1), pode-se obter a distância $d\left(\mathbf{x}^{i}, \mathbf{x}^{j}\right)$ e assim pode-se calcular $k$ instâncias com menores distâncias, que são as mais próximas à instância $\mathbf{x}^{j}$, com valores conhecidos na posição $l$. Estas são chamadas de $k$ vizinhos mais próximos de $\mathbf{x}^{j}$. Após obter o conjunto de $k$ instâncias mais próximas, pode-se estimar os valores ausentes de $x_{l}^{j}$ por meio da média ponderada dos $k$ valores do $l$-ésimo atributo das instâncias vizinhas com $x_{l}^{v} \neq$ ?, $\operatorname{com}(v=1, \ldots, k)$, conforme apresentado na Equação (2.2).

$$
\begin{gathered}
d\left(\mathbf{x}^{i}, \mathbf{x}^{j}\right)=\sqrt{\frac{\sum_{l=1}^{m} r_{l}^{i} \cdot r_{l}^{j} \cdot\left(x_{l}^{i}-x_{l}^{j}\right)^{2}}{\sum_{l=1}^{m} r_{l}^{i} \cdot r_{l}^{j}}} \begin{cases}r_{l}^{i}=0, & \text { se }\left(x_{l}^{i}=?\right) \\
r_{l}^{j}=0, & \text { se }\left(x_{l}^{j}=?\right) \\
r_{l}^{i}=1 & \text { se }\left(x_{l}^{i} \neq ?\right) \\
r_{l}^{j}=1 & \text { se }\left(x_{l}^{j} \neq ?\right)\end{cases} \\
x_{l}^{j}=\sum_{v=1}^{k} w_{v}^{j} \cdot x_{l}^{v}
\end{gathered}
$$

Na Equação (2.2), o termo $w_{v}^{j}$ representa o peso para a $v$-ésima instância vizinha mais próxima à instância com valores ausentes. Este peso está normalizado pelo somatório do inverso das distâncias Euclidianas para os $k$ vizinhos, ou seja, a contribuição do valor conhecido de cada instância vizinha é ponderada pela similaridade a uma instância com valores ausentes, conforme 
Equação (2.3). A imputação por meio do KNNimpute pode ser visualizada no Algoritmo 1.

$$
w_{v}^{j}=\frac{\frac{1}{d\left(\mathbf{x}^{j}, \mathbf{x}^{v}\right)}}{\sum_{v=1}^{k} \frac{1}{d\left(\mathbf{x}^{j}, \mathbf{x}^{v}\right)}}
$$

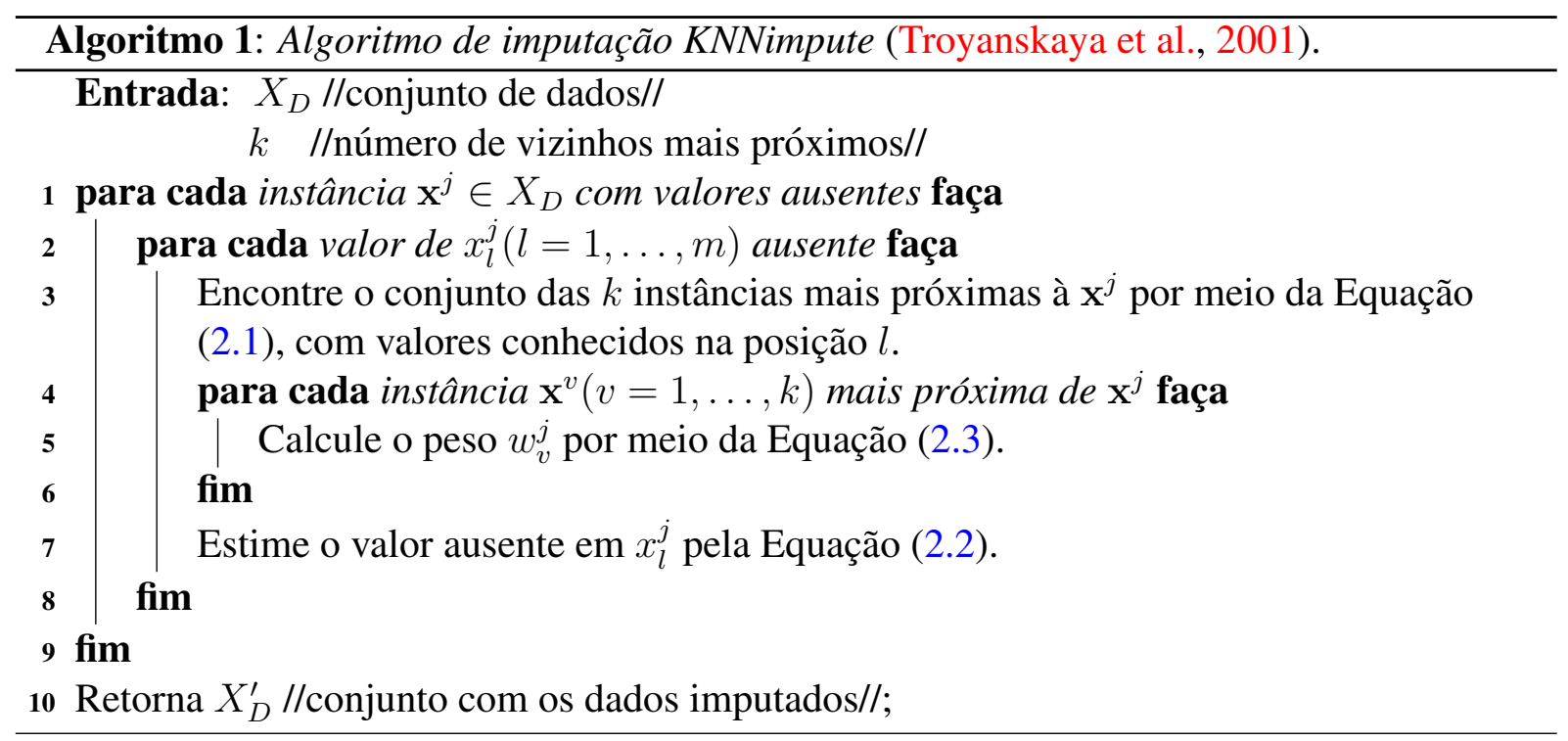

No KNNimpute, o conjunto de instâncias vizinhas é calculado para cada atributo com valor ausente em uma dada instância $\mathbf{x}^{j}$. Dessa forma, o conjunto de $k$ instâncias similares pode conter valores ausentes, exceto para o atributo em que se deseja estimar o valor ausente - neste caso $\mathbf{x}_{l}^{j}$. Consequentemente, o par de vetores referente à uma instância com valores ausentes e uma instância vizinha pode ter tamanhos reais (considerando somente seus valores conhecidos) diferentes. Este problema poderia influenciar nos cálculos de distâncias, favorecendo instâncias com elevadas taxas de valores ausentes, mas é evitado por se utilizar da Equação (2.1). Outra importante característica do KNNImpute envolve a utilização da média dos valores conhecidos ponderada pelo inverso das distâncias para a estimação dos valores ausentes (Equação 2.3). Esta característica favorece a evidência fornecida por instâncias mais similares, dentre as $k$ instâncias mais próximas. Isto porque no conjunto de $k$ vizinhos mais próximos pode haver instâncias que não são tão próximas à instância com valores ausentes, principalmente nos casos em que o número de vizinhos é alto. Por fim, para cada instância imputada, esta não é considerada na estimação dos valores ausentes de outras instâncias. Entretanto, as instâncias com os valores preenchidos podem fornecer informações importantes para a estimação dos valores ausentes das demais instâncias (Kim et al., 2004). Nesse sentido, uma variação deste algoritmo é apresentada na seção seguinte. 


\subsubsection{Algoritmo SKNN (Kim et al., 2004)}

O algoritmo de imputação proposto por Kim et al. (2004), conhecido como Sequential KNN - SKNN, é uma variante do tradicional KNNimpute (Troyanskaya et al., 2001), e possui duas características que o fazem diferir do KNNimpute. A primeira característica é que as instâncias com valores ausentes devem estar ordenadas pela taxa de ausentes, pois os valores ausentes são sequencialmente estimados a partir das instâncias com menores taxas de ausentes. A segunda característica é a reutilização dos dados imputados em imputações posteriores. Dessa forma, o SKNN possibilita que os valores conhecidos das instâncias inicialmente ausentes, bem como seus valores imputados, possam ser úteis para com o processo de imputação de outras instâncias.

$\mathrm{O}$ algoritmo consiste em dividir o conjunto de dados $X_{D}$ em dois subconjuntos: um conjunto $X_{A}$, contendo instâncias com valores ausentes, e outro conjunto $X_{C}$ com instâncias completas (sem valores ausentes em nenhuma tupla). Em seguida, o conjunto $X_{A}$ é ordenado de forma que as instâncias que contêm menores taxas de ausentes ocupem as primeiras posições de $X_{A}$. Um exemplo deste procedimento pode ser visualizado na Figura 2.1. Então, para cada instância do conjunto $X_{A}$ é realizada a imputação dos valores ausentes com os vizinhos mais próximos, atualizando-se do conjunto $X_{C}$, incrementalmente expandido com instâncias imputadas. Os passos de execução do SKNN são descritos no Algoritmo 2. Nesse algoritmo, todos os valores ausentes de uma instância $\mathbf{x}^{i} \in X_{A}$ são imputados com base em um único conjunto de instâncias vizinhas mais próximas de $\mathbf{x}^{i}$, selecionadas do conjunto $X_{C}$. Consequentemente, o algoritmo SKNN oferece uma vantagem em relação ao KNNimpute em termos de custo computacional (Kim et al., 2004), pois o algoritmo KNNimpute calcula várias vezes os $k$ vizinhos mais próximos - uma vez para cada atributo com valor ausente.

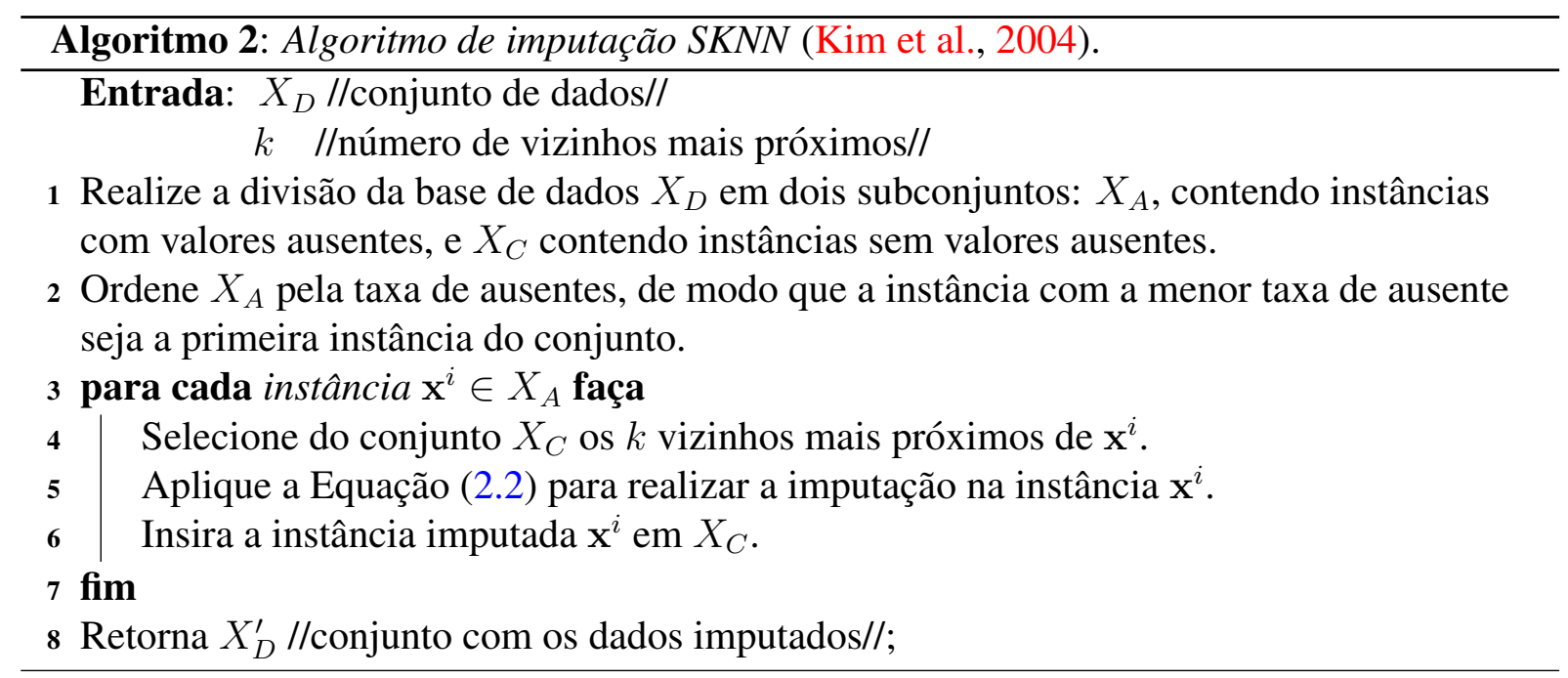

\subsubsection{Algoritmo KNN Iterativo (Brás e Menezes, 2007)}

Brás e Menezes (2007) propuseram um método de imputação iterativo, conhecido como 


\begin{tabular}{|c|c|c|c|c|c|c|c|c|c|}
\hline Instância & $a_{1}$ & $a_{2}$ & $a_{3}$ & $a_{4}$ & 5 & $x_{1}^{5}$ & $x_{2}^{5}$ & $x_{3}^{5}$ & $?$ \\
\hline 1 & $x_{1}^{1}$ & $x_{2}^{1}$ & $x_{3}^{1}$ & $x_{4}^{1}$ & 2 & $x_{1}^{2}$ & $?$ & $x_{3}^{2}$ & $?$ \\
\hline 2 & $x_{1}^{2}$ & $?$ & $x_{3}^{2}$ & $?$ & 7 & $?$ & $x_{2}^{7}$ & $?$ & $?$ \\
\hline 3 & $x_{1}^{3}$ & $x_{2}^{3}$ & $x_{3}^{3}$ & $x_{4}^{3}$ & \multirow{2}{*}{\multicolumn{5}{|c|}{ Matriz $X_{A}$ ordenada pela taxa de ausentes. }} \\
\hline 4 & $x_{1}^{4}$ & $x_{2}^{4}$ & $x_{3}^{4}$ & $x_{4}^{4}$ & & & & & \\
\hline 5 & $x_{1}^{5}$ & $x_{2}^{5}$ & $x_{3}^{5}$ & $?$ & 1 & $x_{1}^{1}$ & $x_{2}^{1}$ & $x_{3}^{1}$ & $x_{4}^{1}$ \\
\hline 6 & $x_{1}^{6}$ & $x_{2}^{6}$ & $x_{3}^{6}$ & $x_{4}^{6}$ & 3 & $x_{1}^{3}$ & $x_{2}^{3}$ & $x_{3}^{3}$ & $x_{4}^{3}$ \\
\hline 7 & $?$ & $x_{2}^{7}$ & $?$ & $?$ & 6 & $x_{1}^{6}$ & $x_{2}^{6}$ & $x_{3}^{6}$ & $x_{4}^{6}$ \\
\hline 8 & $x_{1}^{8}$ & $x_{2}^{8}$ & $x_{3}^{8}$ & $x_{4}^{8}$ & 8 & $x_{1}^{8}$ & $x_{2}^{8}$ & $x_{3}^{8}$ & $x_{4}^{8}$ \\
\hline 9 & $x_{1}^{9}$ & $x_{2}^{9}$ & $x_{3}^{9}$ & $x_{4}^{9}$ & 9 & $x_{1}^{9}$ & $x_{2}^{9}$ & $x_{3}^{9}$ & $x_{4}^{9}$ \\
\hline \multicolumn{5}{|c|}{ Matriz de Dados $X_{D}$. } & \multicolumn{5}{|c|}{ Matriz $X_{C}$ contendo somente instâncias com pletas. } \\
\hline
\end{tabular}

Figura 2.1: Separação da matriz de dados $X_{D}$ em $X_{C}$ (instâncias completas) e $X_{A}$ (instâncias ausentes). A matriz $X_{A}$ está ordenada pela taxa de ausentes.

Iterative KNN Impute (IKNNImpute), reformulando o conceito de reutilização dos dados imputados no processo de estimação dos valores em outras instâncias que apresentem valores ausentes. Este método consiste em recalcular os valores estimados durante o processo de imputação em ciclos. O procedimento inicia-se com a substituição dos valores ausentes pelo método da média, tornando a matriz de dados inicialmente completa. Em seguida, o processo de imputação é realizado iterativamente, durante um determinado número de ciclos, $H$. Para cada ciclo é realizada uma "nova" imputação, que compreende encontrar $k$ instâncias completas mais próximas à instância com valores ausentes e então re-estimar todos os valores com o mesmo conjunto de vizinhos por meio da Equação (2.2). Este processo é repetido até que a soma das diferenças quadráticas entre os valores imputados no ciclo anterior e no ciclo atual $\left(\delta^{h}\right)$ - Equação (2.4) - seja menor do que um limiar $\tau$. Os passos do IKNNImpute estão descritos no Algoritmo 3.

$$
\delta^{h}=\sum_{i=1}^{N} \sum_{l=1}^{m}\left(x_{l}^{i^{(h-1)}}-x_{l}^{i^{(h)}}\right)^{2}
$$

A principal diferença entre o IKNNImpute e o SKNN está na forma de construção do conjunto de dados completos $X_{C}-$ com a reutilização dos dados imputados. No passo 1 do Algoritmo 3 é realizada a substituição dos valores ausentes pela média dos valores conhecidos em cada atributo. A utilização dessa estratégia possibilita que um maior número de instâncias completas possam ser utilizadas para estimar os valores ausentes. Além disso, o procedimento iterativo segue reajustando os valores ausentes estimados em cada ciclo.

\subsubsection{Algoritmo KMI (Hruschka et al., 2004)}

O algoritmo KMI (K-Means Imputation) (Hruschka et al., 2004) é uma variante do método de imputação Hot-deck (Batista, 2003). Este algoritmo se baseia na aplicação prévia de um algoritmo de agrupamento de dados nas instâncias completas, usando as instâncias representativas dos grupos (cluster), tais como centróides, como entrada para a substituição pelo método 


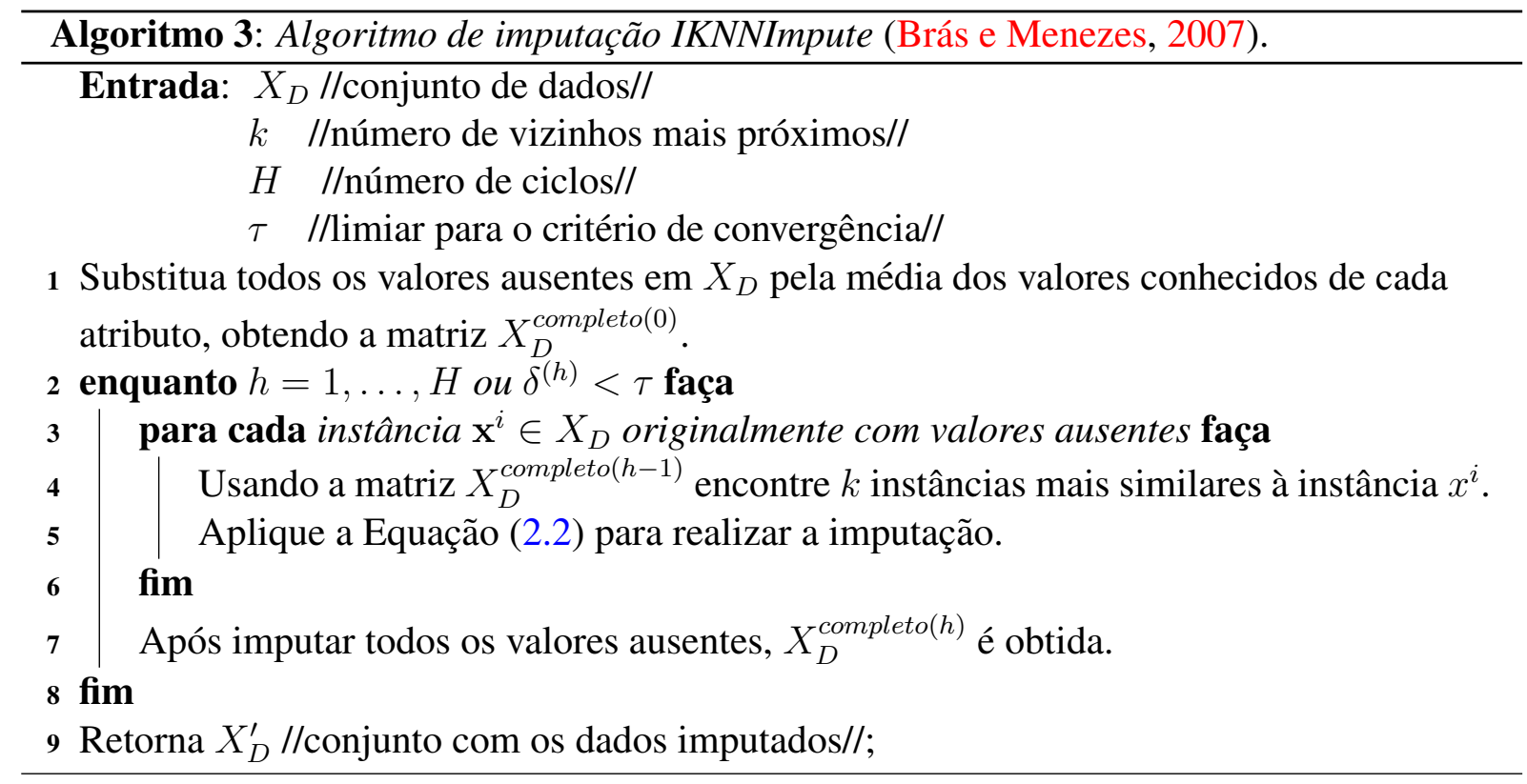

do vizinho mais próximo. Para tal, podem-se empregar algoritmos clássicos de agrupamento, tal como o algoritmo das $K$-médias, que consiste em particionar um conjunto de dados de $N$ instâncias em $K$ grupos, minimizando a soma das distâncias entre as instâncias e seus correspondentes centróides. Essa idéia pode ser posta em prática por meio do algoritmo KMI (Hruschka et al., 2004).

O algoritmo KMI inicia-se com a obtenção de $K$ centróides (vetores médios) por meio da aplicação algoritmo das $K$-médias na matriz de dados contendo instâncias com valores completos $\left(X_{C}\right)$. Após obter os centróides, estes são utilizados para que o algoritmo $k$-NN (neste caso, $k=1$ ) encontre a instância completa mais próxima (neste caso, um centróide) à uma instância com valores ausentes. Em seguida, a imputação consiste em utilizar os valores do centróide para a substituição dos valores ausentes, conforme descrito no Algoritmo 4. Neste algoritmo é utilizada a Equação (2.1) para calcular a distância entre uma instância com valores ausentes e um centróide (que não possui valores ausentes).

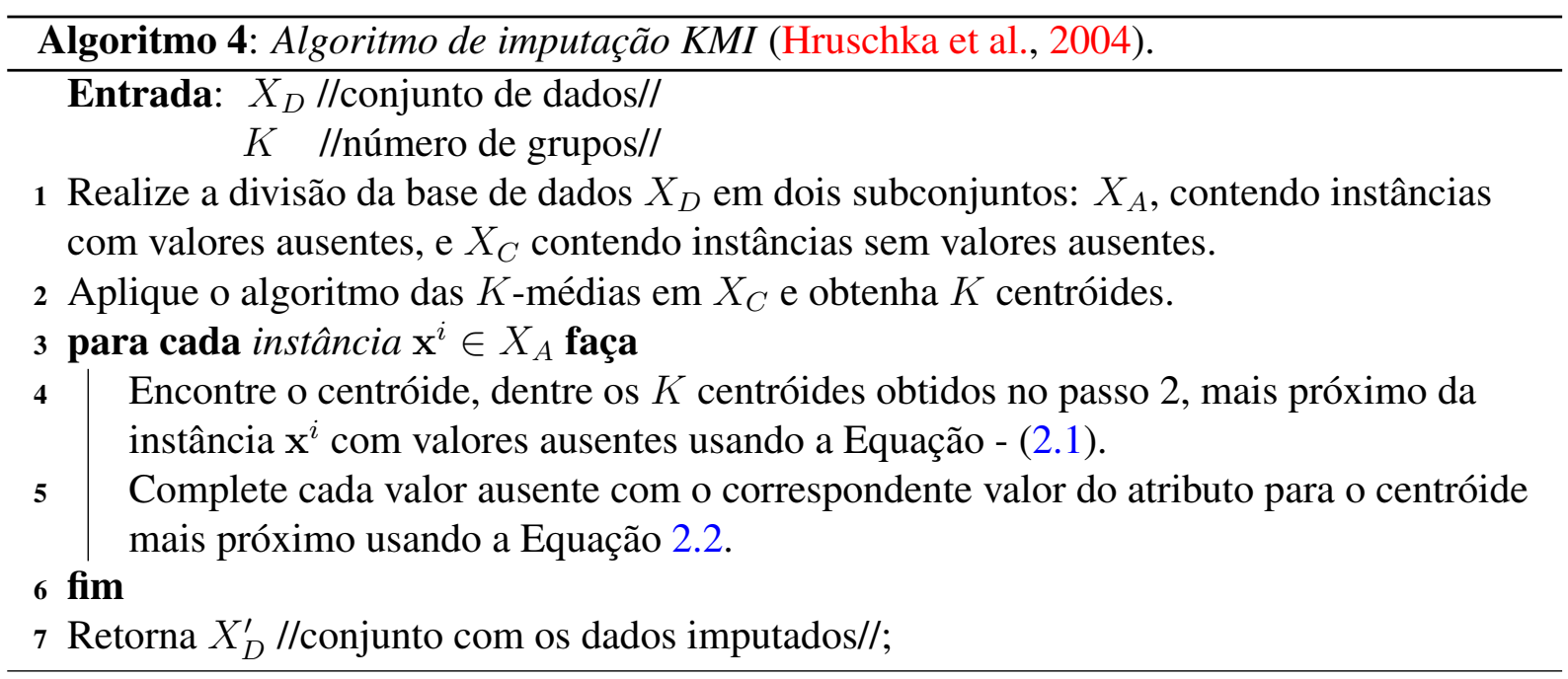


O KMI pode proporcionar resultados promissores e pode permitir um aumento significativo da eficiência computacional, conforme demonstrado em Hruschka et al. (2004). No entanto, o Algoritmo KMI requer que o usuário defina o número de grupos a priori, e este número deve ser relativamente baixo para que o algoritmo seja computacionalmente mais eficiente do que o tradicional algoritmo KNNImpute (Hruschka et al., 2004).

\subsubsection{Considerações sobre os Algoritmos de Imputação Baseados em Vi- zinhos Mais Próximos}

Apesar dos métodos de imputação baseados no $k$-NN serem amplamente utilizados, estes sofrem de algumas limitações, tais como (Acuna e Rodriguez, 2004):

- Necessidade da escolha da função de distância, por exemplo, Euclidiana ou Mahalanobis.

- Definição do número de vizinhos mais próximos.

- Necessidade de consultar toda a base de dados para calcular os vizinhos mais próximos para cada instância com valores ausentes, implicando em custos computacionais significativos.

Em muitos métodos de imputação é comumente utilizada a função de distância Euclidiana (Troyanskaya et al., 2001; Batista e Monard, 2003; Kim et al., 2004; Hruschka et al., 2004; Brás e Menezes, 2007; Kim et al., 2007). Em Troyanskaya et al. (2001) foram realizados experimentos controlados para avaliar diferentes medidas de similaridade (correlação de Pearson, distância Euclidiana e minimização da variância) e os autores concluíram que a distância Euclidiana é suficientemente adequada. Além disso, a sensibilidade do parâmetro $k$ foi analisada e os autores sugerem $k=10$, desde que existam pelo menos 10 vizinhos mais próximos a uma instância com valores ausentes.

Em relação à necessidade de se calcular a distância entre uma instância com valores ausentes a todas as instâncias da base de dados, uma possível solução seria utilizar uma representação compacta ou realizar a sumarização da base de dados. Dessa forma, o número de operações para cálculo da distância pode ser significativamente reduzido. Uma abordagem possível para sumarizar dados consiste do uso de técnicas de agrupamento de dados. Na seção 2.3.4 foi apresentado um algoritmo de imputação - KMI (Hruschka et al., 2004) - baseado no bem conhecido algoritmo de agrupamento das $K$-médias (Wu et al., 2007). Porém, este algoritmo é dependente da definição prévia do número de grupos que, embora tenha sido extensivamente estudada na literatura, é uma tarefa difícil (Sheng et al., 2003; Kaufman e Rousseeuw, 1990).

De modo geral, as principais limitações dos algoritmos descritos consistem em determinar o número de vizinhos mais próximos e o número de grupos. Conforme relatado em Troyanskaya et al. (2001), a seleção ótima do número de vizinhos mais próximos provavelmente depende do tamanho dos grupos em um determinado conjunto de dados. Dessa forma, a identificação 
de grupos em um conjunto de dados pode auxiliar na definição do número ideal de vizinhos mais próximos. Neste caso, surge o problema de identificar o número de grupos em um conjunto de dados. Uma possível abordagem para solucionar esse problema consiste na utilização de estratégias evolutivas para estimar automaticamente o número de grupos (Hruschka et al., 2006). Neste trabalho, um algoritmo evolutivo para enfrentar tais problemas é apresentado. Tal algoritmo é objeto de consideração no próximo Capítulo. 


\section{EACImpute: Algoritmo Evolutivo para Imputação Baseado em Agrupamento}

Este capítulo está organizado da seguinte forma. Na Seção 3.1 são descritos conceitos introdutórios sobre algoritmos evolutivos que são necessários para a compreensão do algoritmo aqui proposto. Na Seção 3.2 descreve-se o algoritmo evolutivo EAC ${ }^{1}$ (Hruschka et al., 2006), o qual serviu de base para o algoritmo de imputação proposto, descrito na Seção 3.3.

\subsection{Considerações Iniciais}

Algoritmos evolutivos são métodos de busca estocásticos baseados no processo de evolução de Darwin (Freitas, 2002). Esses algoritmos basicamente atuam em uma população de genótipos, na qual cada genótipo representa uma solução para um determinado problema. A cada iteração (geração) os genótipos mais aptos, avaliados de acordo com uma função de adequabilidade, são probabilisticamente selecionados para dar origem a novos genótipos e formarem a população da geração seguinte. Esse processo é repetido iterativamente até que um critério de convergência seja satisfeito, tal como o número de gerações executadas ou até que uma solução satisfatória seja encontrada (Maimon e Rokach, 2005). De maneira específica, algoritmos evolutivos tendem a evitar mínimos locais ao adotar uma amostragem probabilística, porém guiada (por meio da função de adequabilidade), para se realizar busca no espaço de soluções (população).

Em Hruschka et al. (2009a) é apresentada uma extensa revisão da literatura abordando

\footnotetext{
${ }^{1}$ Neste trabalho será adotada a sigla EAC usada para identificar o algoritmo cujo nome original foi proposto na língua Inglesa (Evolutionary Algorithm for Clustering) por simplicidade e para preservar sua denominação original.
} 
possíveis estratégias para a utilização de algoritmos evolutivos em problemas de agrupamento, sob a perspectiva de seis componentes: esquema de codificação, operadores, população, função de adequabilidade, seleção e procedimento de busca local. O esquema de codificação está relacionado à estrutura adotada para a representação da solução, denominada de genótipo. Uma população consiste de um conjunto de possíveis soluções (partições de dados) que podem ser representadas como pontos no espaço de busca. A função de adequabilidade usualmente avalia cada partição por meio de um critério de validação do agrupamento. A seleção consiste basicamente de um procedimento probabilístico para selecionar partições (soluções) que melhor se adequam ao propósito de agrupar os dados. Esta seleção é guiada pelo valor atribuído à partição por meio da função de adequabilidade. Os operadores atuam nas partições selecionadas para gerar novas soluções por meio de procedimentos de cruzamento e mutação. Os operadores e o procedimento de seleção são aplicados na população para modificar as soluções existentes e formar uma "nova" população. Por fim, procedimentos de busca local podem ser aplicados para realizar um refinamento nas partições.

\subsection{EAC}

Em linhas gerais, o EAC realiza uma amostragem probabilística das partições obtidas pelo algoritmo das $K$-médias, variando-se tanto o valor de $K$ quanto a inicialização, por meio de um processo de busca informada. O algoritmo foi projetado de tal forma a fornecer uma sinergia entre o algoritmo das $K$-médias e os operadores evolutivos do EAC, tendendo a minimizar a variância dos grupos obtidos. Tais operadores manipulam os grupos (clusters) obtidos durante a busca evolutiva eliminando, dividindo e reagrupando clusters. Esta manipulação dos clusters pode solucionar dois problemas do algoritmo das $K$-médias (Everitt et al., 2001): i) dependendo da inicialização, o algoritmo pode ficar preso em soluções subótimas (mínimos locais); e ii) o número de grupos $(K)$ precisa ser definido a priori. Os passos principais do EAC são descritos no Algoritmo 5 e serão detalhados nas seções seguintes.

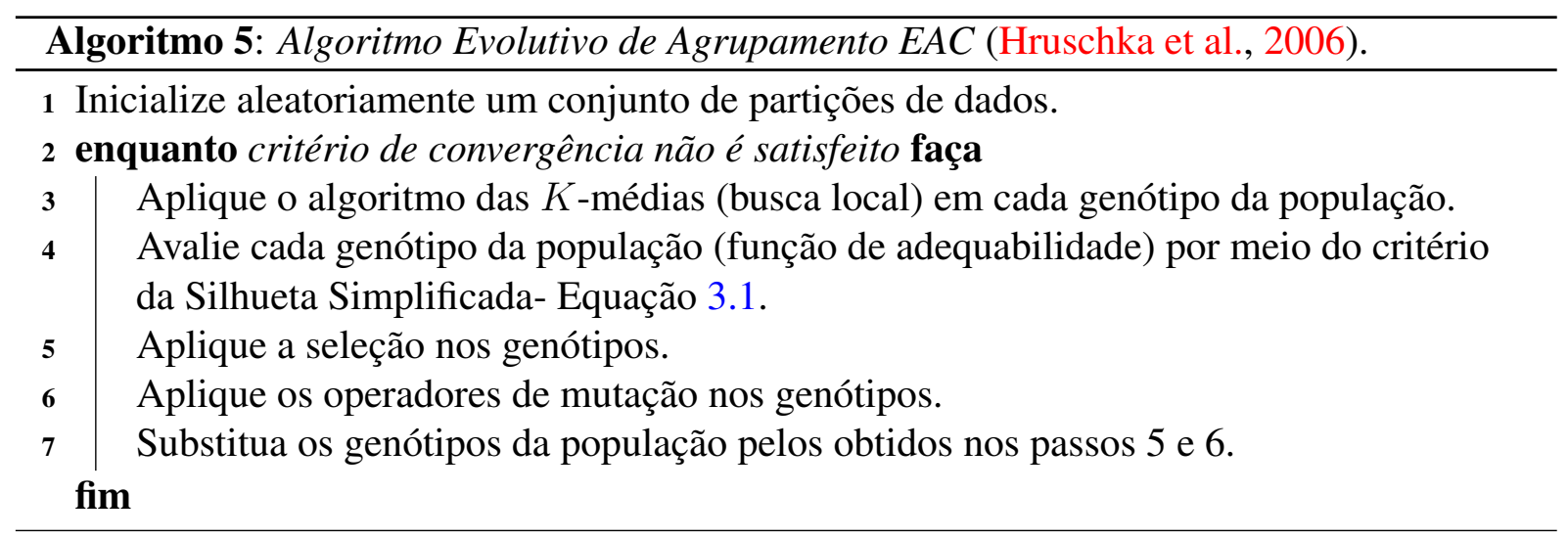




\subsubsection{Esquema de Codificação}

O esquema de codificação da solução (partição) envolve um vetor de números inteiros, no qual o valor de cada elemento refere-se a um grupo e o índice do vetor refere-se à uma instância. Para exemplificar o esquema de codificação, considere uma base de dados em duas dimensões $\left(\left\{a_{1}, a_{2}\right\}\right)$ composta de 12 instâncias particionadas em 3 grupos, conforme ilustrado na Figura 3.1. Um genótipo u que representa a partição para esses dados é apresentado na Figura 3.2. Nesse exemplo, quatro instâncias $\{1,2,11,12\}$ recebem os valores de rótulo referente ao $\mathrm{Grupo}_{1}-$ neste caso, o valor 1 . A posição de cada elemento do genótipo corresponde ao índice de uma instância na base dados. Esses índices são apresentados ao lado dos símbolos da Figura 3.1. O último elemento do vetor contém o número de grupos $(K)$ que a partição está codificando.

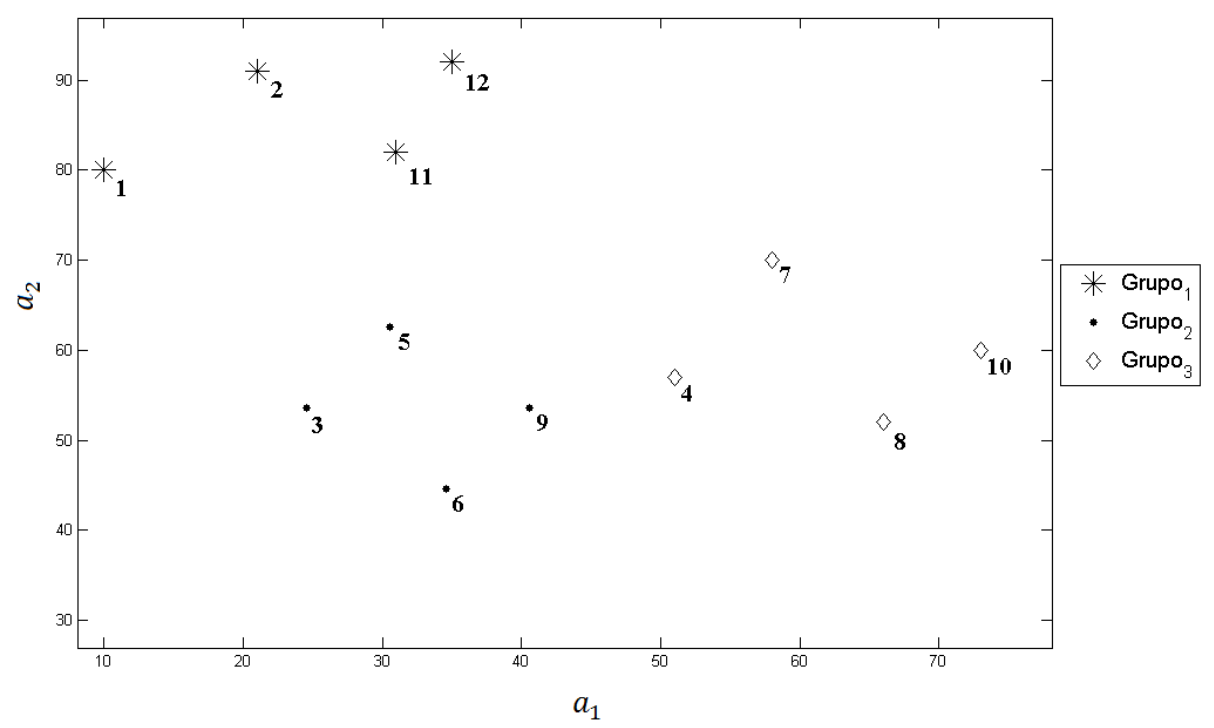

Figura 3.1: Representação gráfica dos dados: eixos representam atributos e símbolos representam grupos.

\begin{tabular}{|c|c|c|c|c|c|c|c|c|c|c|c|c|c|c|}
\hline $\mathbf{u}=$ & 1 & 1 & 2 & 3 & 2 & 2 & 3 & & 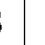 & 2 & 3 & 1 & 1 & 3 \\
\hline
\end{tabular}

Figura 3.2: Representação genotípica da partição apresentada na Figura 3.1. O elemento de índice 13 contém o número de grupos codificados.

\subsubsection{Processo de Busca Local}

O processo de busca local tem como objetivo refinar as soluções obtidas durante a busca evolutiva e acelerar a convergência do EAC. Esse processo é realizado por meio do popular algoritmo das $K$-médias que é aplicado em cada genótipo da população. Dessa forma, o algoritmo das $K$-médias realiza um refinamento nas partições, minimizando a soma das distâncias 
(e.g., Euclidiana) entre as instâncias dos grupos e seus respectivos centróides, iterativamente até que um critério de parada seja satisfeito.

\subsubsection{Função de Adequabilidade}

A função de adequabilidade padrão adotada no EAC (Hruschka et al., 2006) é baseada na silhueta proposta por Kaufman e Rousseeuw (1990), denominada de Silhueta Simplificada. Essa função é aplicada para mensurar a qualidade das partições obtidas, isto é, para avaliar cada genótipo da população. Para melhor compreensão, considere um objeto $\mathrm{x}^{i}$ associado a um grupo, por exemplo, $\mathbf{G}_{a}$. Então, calcula-se a distância entre a instância $x^{i}$ e o centróide do seu grupo $\left(\mathbf{G}_{a}\right)$, obtendo-se o valor de $a\left(\mathbf{x}^{i}\right)$. Em seguida, calcula-se a distância entre a instância $\mathrm{x}^{i}$, pertencente ao grupo $\mathrm{G}_{\mathrm{a}}$, para o centróide do grupo mais próximo $\left(\mathrm{G}_{\mathrm{b}}\right)$, obtendo-se o valor de $b\left(\mathbf{x}^{i}\right)=\min \left[d\left(\mathbf{x}^{i}, \mathbf{G}_{\mathbf{b}}\right)\right]$, tal que $\mathbf{G}_{\mathbf{b}} \neq \mathbf{G}_{\mathbf{a}}$. Dessa maneira, a Silhueta Simplificada $s\left(\mathbf{x}^{i}\right)$ é dada por:

$$
s\left(\mathbf{x}^{i}\right)=\frac{b\left(\mathbf{x}^{i}\right)-a\left(\mathbf{x}^{i}\right)}{\max \left[a\left(\mathbf{x}^{i}\right), b\left(\mathbf{x}^{i}\right)\right]}
$$

Percebe-se por meio da Equação (3.1) que o valor de $s\left(\mathbf{x}^{i}\right)$ está contido no intervalo $[-1,1]$. Quanto maior o valor de $s\left(\mathbf{x}^{i}\right)$ melhor alocado está o objeto $\mathbf{x}^{i}$ ao grupo $\mathbf{G}_{1}$. Se o valor de $s\left(\mathbf{x}^{i}\right)$ é igual a zero, então não está bem claro para qual grupo esta instância deveria pertencer, seja o grupo atual ou o grupo mais próximo. Finalmente, se o grupo $\mathrm{G}_{1}$ é unitário (singleton), então $s\left(\mathbf{x}^{i}\right)$ não é definido e a escolha mais conservadora é atribuir $s\left(\mathbf{x}^{i}\right)=0$ (Kaufman e Rousseeuw, 1990). No EAC cada partição $o^{j}-$ para $1 \leq j \leq P$, sendo que $P$ é o tamanho da população, é avaliada pela média dos valores de $s\left(\mathbf{x}^{i}\right)$ para cada instância da base de dados, conforme apresentado na Equação 3.2.

$$
\bar{S}\left(o^{j}\right)=\frac{1}{N} \cdot \sum_{i=1}^{N} s\left(\mathbf{x}^{i}\right) \quad \text { para } \quad 1 \leq j \leq P
$$

\subsubsection{Seleção}

O processo de seleção consiste na escolha dos genótipos que serão submetidos aos operadores de mutação. Este processo tipicamente realiza uma seleção probabilística baseada nos valores de adequabilidades dos genótipos. A estratégia de seleção utilizada no EAC consiste na tradicional estratégia da roleta (seleção proporcional) (Eiben e Smith, 2003). Esta estratégia não admite valores de adequabilidade negativos. Dessa maneira, cada genótipo tem seu valor de adequabilidade normalizado linearmente (Davis, 1996). Adicionalmente, uma estratégia elitista é utilizada para manter o melhor genótipo para a próxima geração (Fogel, 1999). 


\subsubsection{Operadores de Mutação}

Os operadores de mutação são caracterizados por serem estocásticos, pois dependem de uma série de escolhas aleatórias (Eiben e Smith, 2003). Estes operadores atuam em uma sequiência de passos elementares dentro de um espaço de busca. No EAC, dois operadores de mutação que envolvem divisão e remoção de grupos são utilizados. O operador de remoção é aplicado em $50 \%$ da população e o operador de divisão nos genótipos restantes.

\subsubsection{Operador de Remoção}

O operador de remoção é aplicado nos genótipos que codificam mais que dois grupos. Em conformidade com esta condição, esse operador seleciona aleatoriamente $s \in\{1, \ldots, K-2\}$ grupos a serem removidos de maneira que restem pelo menos 2 grupos na partição (genótipo) - Algoritmo 6. Em seguida, para cada instância de um grupo selecionado $G_{r}, 1 \leq r \leq s$ calculam-se as distâncias desta aos centróides dos grupos restantes. Por meio dessas distâncias, as instâncias do grupo selecionado são atribuídas aos grupos mais próximos.

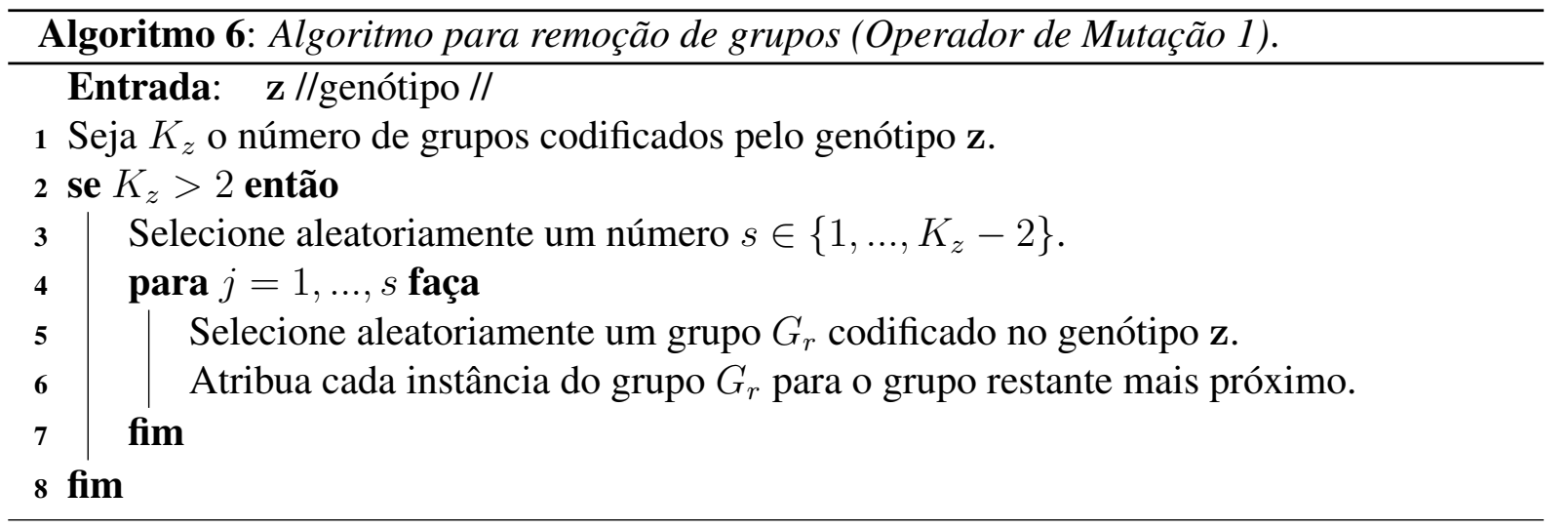

Para ilustrar o procedimento de execução desse operador, considere o exemplo apresentado na Figura 3.3. Nesta figura, são apresentados 3 grupos representados pelos símbolos: “*”

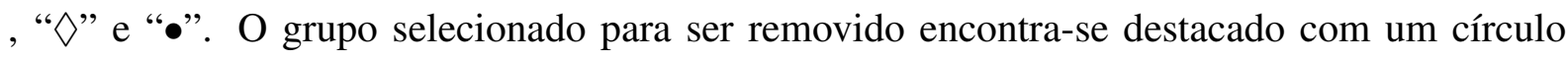
envolvendo as instâncias representadas pelo símbolo “•”. Para cada instância desse grupo selecionado, calculam-se as distâncias destas aos centróides restantes, representados pelo símbolo “”. Com base nessas distâncias, as instâncias são alocadas aos grupos mais próximos.

\subsubsection{Operador de Divisão}

O segundo operador de mutação realiza a divisão de grupos que contêm mais que uma instância. Como exemplo ilustrativo do processo, considere a Figura 3.4. Nesse exemplo, o operador seleciona aleatoriamente um grupo para ser dividido, representado por um círculo que envolve as instâncias representadas pelo símbolo “ $*$ ’. Em seguida, seleciona-se aleatoriamente uma instância $\mathbf{x}^{i}$ do grupo a ser dividido (Figura 3.4(a)). Outra instância $\mathbf{x}^{j}$, mais distante de $\mathbf{x}^{i}$, é selecionada (Figura 3.4(b)). Essas duas instâncias $\left(\mathrm{x}^{i} \mathrm{e} \mathrm{x}^{j}\right)$ representam os protótipos de 


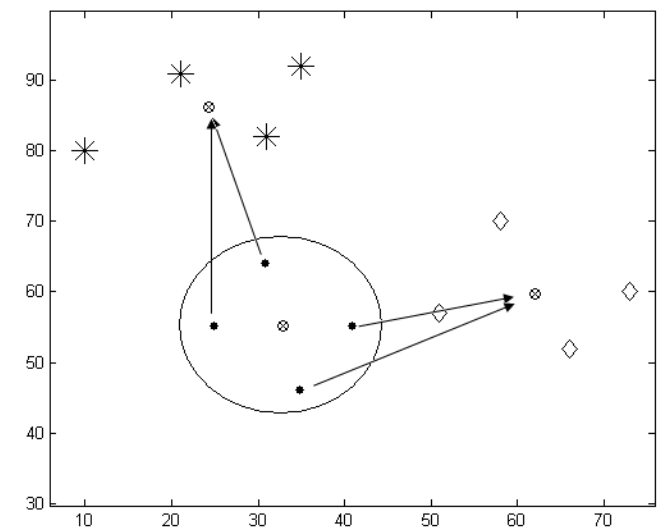

(a) Selecionando aleatoriamente um grupo e encontrando os grupos mais próximos às instâncias do grupo selecionado.

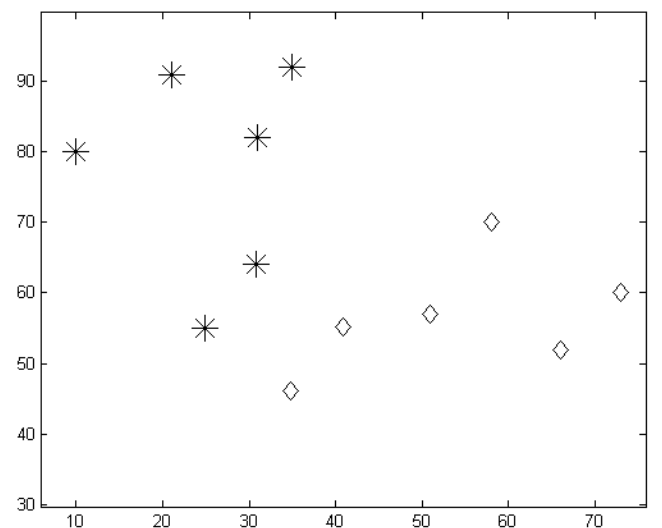

(b) Resultado da aplicação do operador de remoção de grupos.

Figura 3.3: Aplicação do operador de mutação para remoção de grupos.

dois novos grupos. Por fim, encontram-se as instâncias do grupo selecionado mais próximas às instâncias $\mathbf{x}^{i}$ e $\mathrm{x}^{j}$ formando dois novos grupos. Os passos de execução do operador de divisão estão descritos no Algoritmo 7.

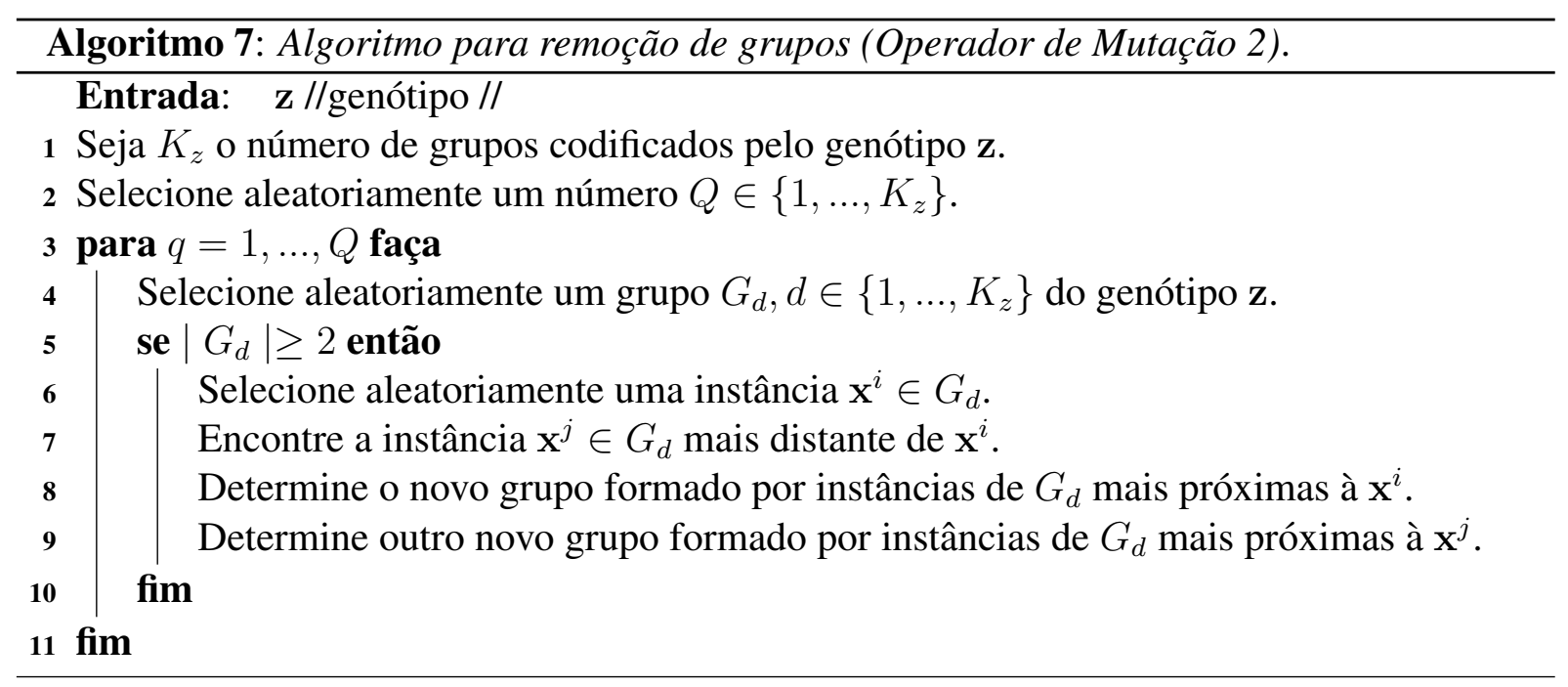




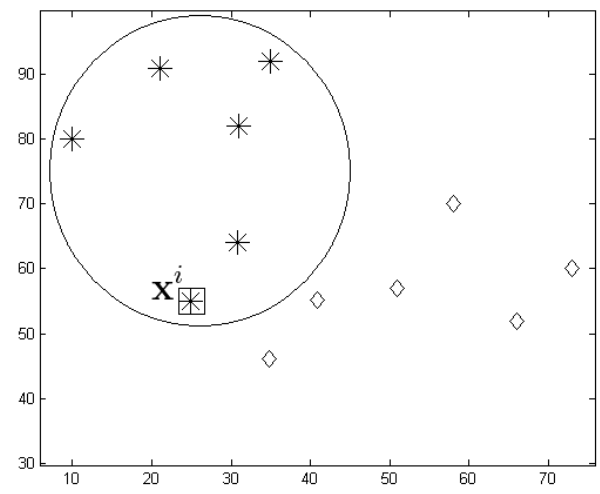

(a) Selecionando aleatoriamente um grupo e uma instância.

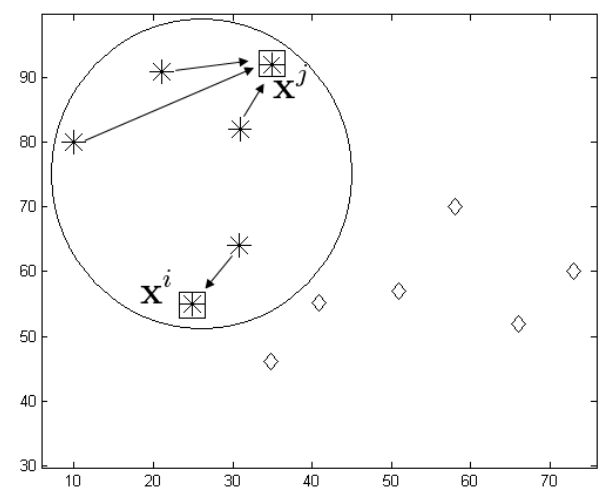

(c) Encontrando as instâncias mais próximas às duas instâncias selecionadas.

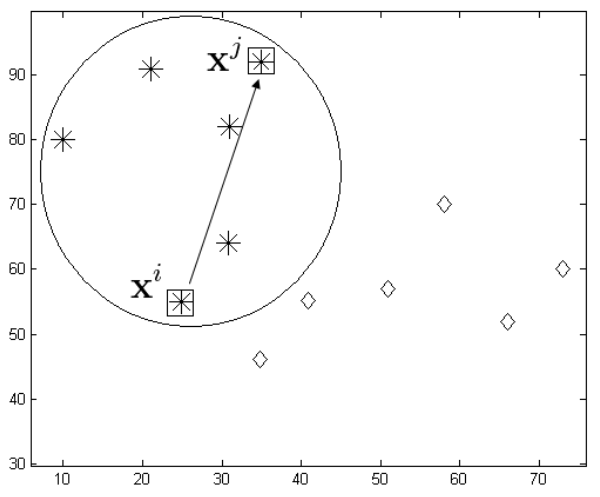

(b) Encontrando a instância mais distante da instância selecionada.

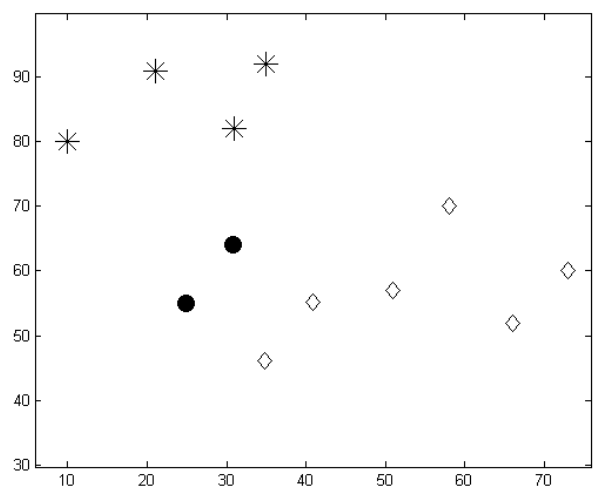

(d) Dois novos grupos gerados.

Figura 3.4: Aplicação do operador de mutação para divisão de grupos.

\subsection{EACImpute}

A idéia básica do EACImpute é iterativamente realizar o agrupamento dos dados, considerando também instâncias com valores ausentes, e executar a imputação. O processo de imputação segue refinando os valores estimados a cada geração (iteração), conforme apresentado no Algoritmo 8. As principais modificações realizadas no EAC para lidar com valores ausentes consistem basicamente em:

a) No passo 3, bem como nos passos 4, 6 e 7, o cálculo de dissimilaridade (Euclidiana) entre instâncias é tolerante aos valores ausentes. Resumidamente, apenas pares de valores conhecidos são usados para calcular a dissimilaridade entre duas instâncias (ou entre uma instância e um centróide). Para evitar falsas distâncias baixas para um instância com valores ausentes, a média de cada dissimilaridade calculada é ponderada pela quantidade de pares de valores conhecidos.

b) No passo 4 , uma matriz de dados preenchida $\mathbf{X}^{(t)}{ }_{P}$ é derivada dos valores estimados por preencher os valores ausente de $\mathbf{X}_{A}$. Tais valores serão aqui denominados de estimativas (ou alternativamente, imputações parciais) porque estes valores provavelmente variam 
durante as gerações $(t=1, \ldots, G)$. Dessa maneira, os valores estão realmente imputados quando $t=G$. Este passo de imputação será detalhado na Seção 3.3.1.

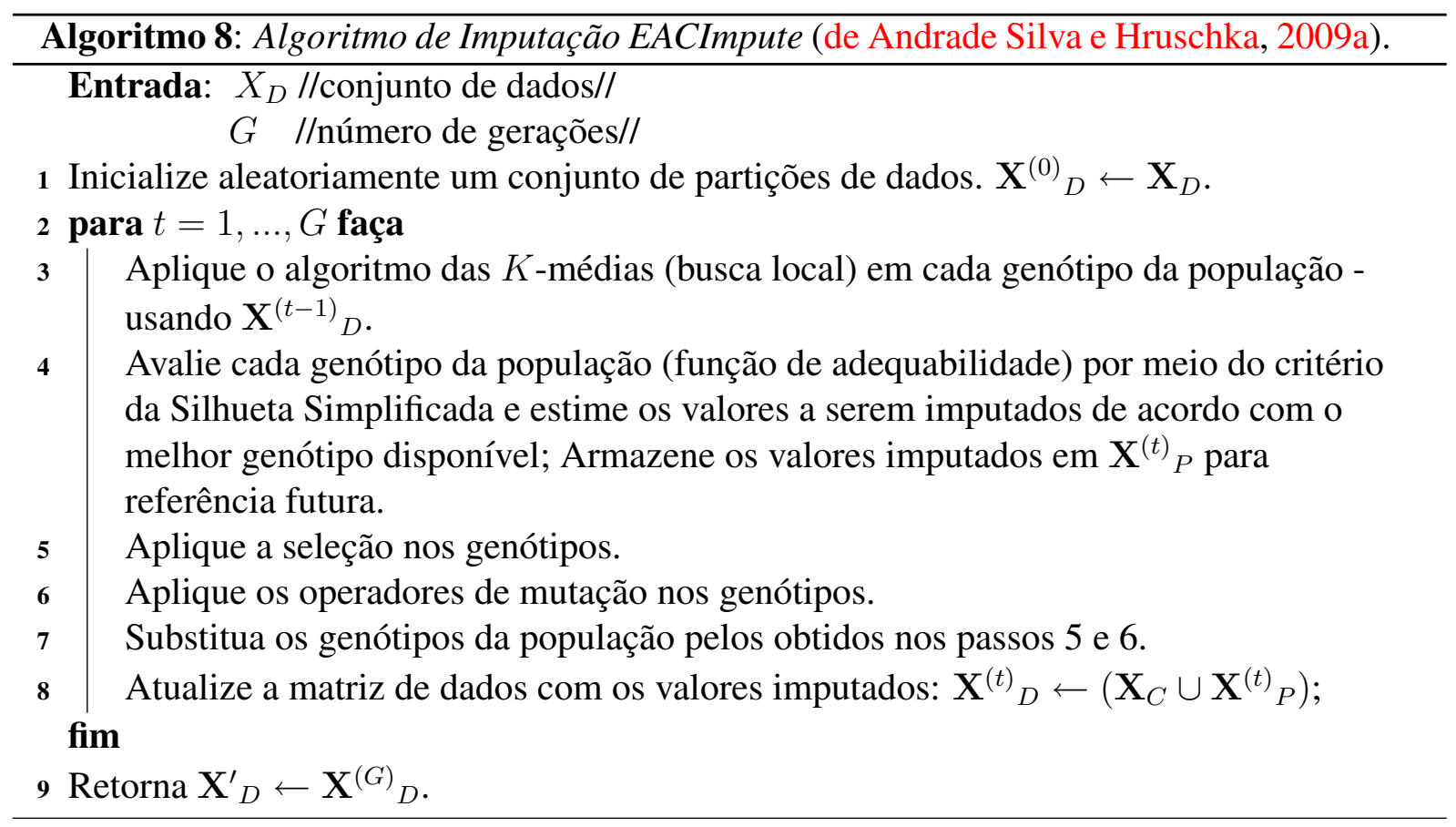

\subsubsection{Imputação}

O EACImpute reúne estratégias adotadas por algoritmos de imputação amplamente utilizados na literatura (apresentados no Capítulo 2). Essas estratégias consistem na aplicação do processo de imputação baseado no $k$-NN (sugerido no KNNImpute (Troyanskaya et al., 2001)), na reutilização dos valores imputados no processo de estimação dos valores ausentes (implementado no SKNN (Kim et al., 2004)), na aplicação de um processo iterativo para refinar os valores estimados (implementado no IKNNImpute (Brás e Menezes, 2007)) e na aplicação de um processo de agrupamento (implementado no KMI (Hruschka et al., 2004)). Apesar de herdar as características desses algoritmos, o EACImpute não apresenta parâmetros críticos tais como a definição do número de vizinhos $(k)$ e o número de grupos $(K)$.

O processo de imputação inicia-se com a escolha de uma partição para auxiliar na estimação dos valores ausentes da base de dados. Como o EACImpute trabalha com um conjunto (população) de partições de dados (genótipos), a escolha natural seria utilizar a partição que apresenta o melhor valor de silhueta na geração atual. Assim sendo, essa partição é utilizada como entrada para o processo de imputação. A imputação é realizada em cada grupo de dados codificados na partição. Para cada grupo $G_{i}, 1 \leq i \leq K$, contendo instâncias com valores ausentes, são calculadas as distâncias das instâncias com valores ausentes a todas as instâncias do grupo, inclusive as instâncias que contém valores ausentes (ver Equação 2.1). Em seguida, a estimação dos valores ausentes se dá por meio da Equação 2.2, em que $k=\left|G_{i}\right|$. Conforme 
discutido no Capítulo 2, os valores estimados por essa equação são ponderados pelo inverso da distância de maneira que as instâncias mais próximas às instâncias com valores ausentes têm um peso maior na imputação.

Para ilustrar o processo de execução do EACImpute, considere a Figura 3.5. Nesta figura, são apresentados os principais passos do algoritmo, que consistem na escolha do melhor genótipo para aplicação do processo de imputação em cada grupo de dados e a realização dos processos de seleção, mutação, atualização da matriz de dados, aplicação do algoritmo das $K$-médias e os cálculos de adequabilidade em cada geração. Considere então um conjunto de dados com 12 instâncias descrito por 4 atributos $-a_{l}(1 \leq l \leq 4)$ - e uma população formada de por 6 genótipos, cada qual codificando diferentes partições de dados. Cada genótipo possui um valor para a função de adequabilidade (Silhueta Simplificada). De acordo com o valor de adequabilidade, o sexto genótipo (de cima para baixo) codifica a melhor solução de agrupamento (dois grupos) para esta população (maior valor de adequabilidade, i.e., 1,86) e, por este motivo, será utilizado para o propósito de imputação. As imputações são realizadas por meio do princípio dos vizinhos mais próximos, adotado pelos algoritmos descritos no Capítulo 2, que considera todas as instâncias que contêm valores conhecidos em um determinado atributo para imputar seus valores em uma dada instância que contém valores ausentes. Esse procedimento é realizado em cada grupo (neste caso, codificado pelo sexto genótipo). Para ilustrar esse procedimento, considere a substituição do valor ausente do segundo atributo $\left(a_{2}\right)$ da instância de $I D=1$ pertencente ao grupo 1 . O valor ausente dessa instância será imputado considerando os valores conhecidos das instâncias pertencentes ao grupo 1 (i.e., instâncias cujo $I D$ 's são $0,2,3,4$ e 5 ). Este método determina automaticamente o número de vizinhos (neste caso, 5 vizinhos), cuja definição pode ser difícil de ser realizada em prática por outras ferramentas de imputação. De maneira análoga, os dois valores ausentes da instância de $I D=4$ serão imputados considerando os valores conhecidos das instâncias cujo os $I D$ 's são $0,1,2,3$ e 5 . Após a imputação o processo evolutivo (seleção, mutação, etc.) é repetido.

$\mathrm{Na}$ eventualidade de algum grupo conter apenas instâncias com valores ausentes nos mesmos atributos, o processo de imputação não atuará neste grupo devido à falta de informação necessária para a estimação dos valores ausentes, ficando a cargo do processo evolutivo tratar esse caso para que seja possível estimar os valores ausentes desses grupos. Para lidar com essa situação anômala, a função de adequabilidade atribui, para cada instância que pertence ao grupo que contém valores ausentes no mesmo atributo, o valor de silhueta $s\left(\mathbf{x}^{i}\right)$ igual a -1 . Dessa maneira, a partição que contém grupos que apresentam essas características possui menor valor de adequabilidade para reduzir a possibilidade de seleção dessa partição (genótipo) por meio do critério da roleta. Caso esta partição seja selecionada, o operador de remoção tem prioridade em atuar neste grupo para removê-lo e adicionar suas instâncias aos grupos mais próximos, que provavelmente contêm informações necessárias para a estimação dos valores ausentes dessas instâncias. 
População (partição de dados)

$[1,1,1,1,3,1,2,3,3,2,3,2$ ] 3$]$

$[1,1,3,1,1,3,2,2,3,2,4,4 \mid 4]$

$[1,1,1,2,2,2,1,1,2,2,1,2 \mid 2]$

$[1,1,1,1,2,2,1,3,3,2,2,3$ 3]

$[1,1,1,1,1,2,2,1,1,2,2,1 \mid 2]$

$[1,1,1,1,1,1,2,2,2,2,2,2 \mid 2]$

$\uparrow$
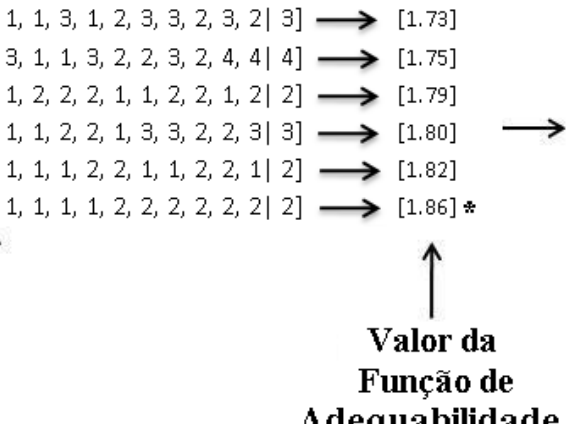

$\rightarrow[1.75]$

$\rightarrow[1.79]$

[1.82]

equabilidade

\begin{tabular}{|c|c|c|c|c|c|}
\hline ID & $a_{1}$ & $a_{2}$ & $a_{3}$ & $a_{4}$ & Grupo \\
\hline 0 & 5.1 & 3.5 & 1.4 & 0.2 & 1 \\
\hline 1 & 4.9 & $?$ & 1.4 & 0.2 & 1 \\
\hline 2 & 4.6 & 3.1 & 1.5 & 0.2 & 1 \\
\hline 3 & 5.0 & 3.6 & 1.4 & 0.2 & 1 \\
\hline 4 & 5.4 & 3.9 & $?$ & $?$ & 1 \\
\hline 5 & 5.7 & 4.4 & 1.5 & 0.4 & 1 \\
\hline 6 & 6.7 & 3.1 & 4.7 & 1.5 & 2 \\
\hline 7 & 6.3 & 2.3 & 4.4 & 1.3 & 2 \\
\hline 8 & 5.6 & 3.0 & 4.1 & 1.3 & 2 \\
\hline 9 & 5.5 & 2.5 & 4.0 & 1.3 & 2 \\
\hline 10 & $?$ & 2.6 & 4.4 & 1.2 & 2 \\
\hline 11 & 5.8 & 2.6 & 4.0 & 1.2 & 2 \\
\hline
\end{tabular}

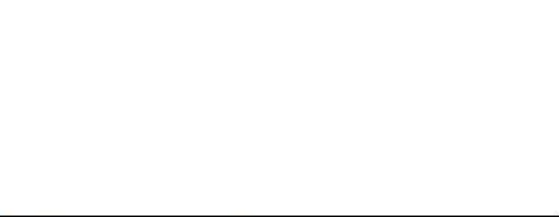

1) Seleção do genótipo;

2) Mutação;

Nova população;

3) Atualização da matriz (imputações);

4) K-Médias;

5) Adequabilidade.

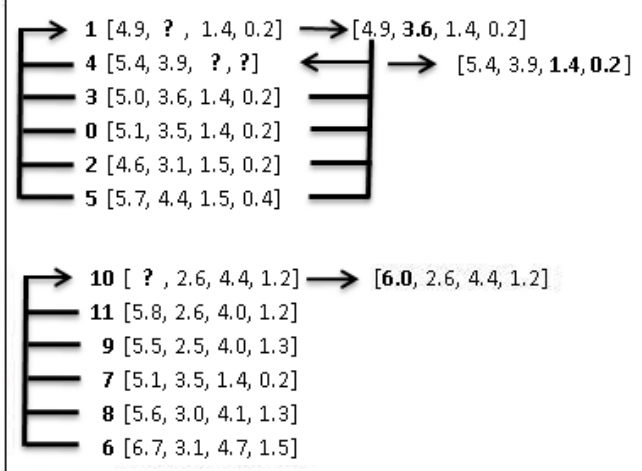

Figura 3.5: Processo ilustrativo da imputação executada no EACImpute. 


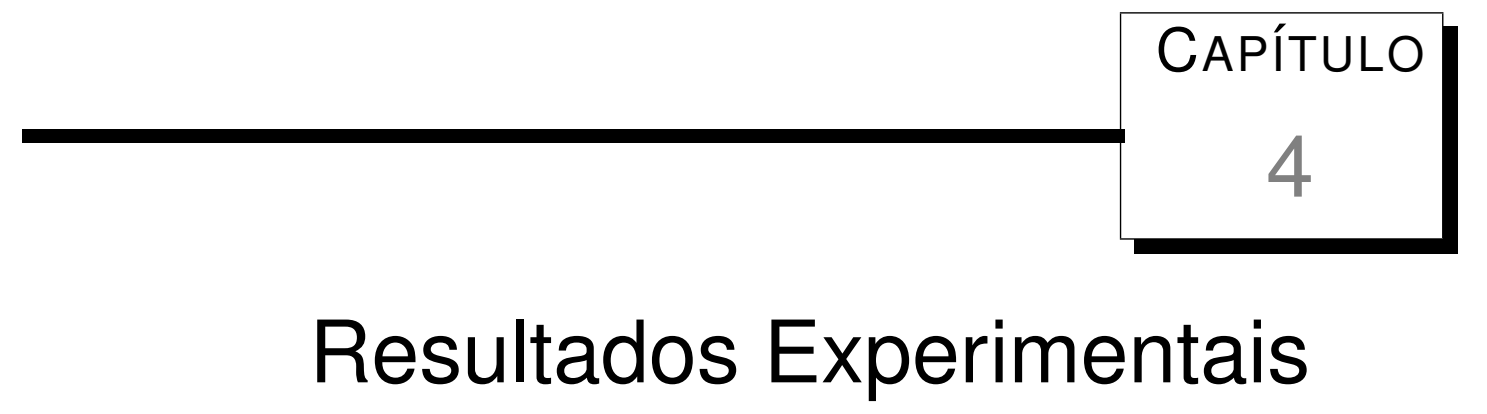

Neste Capítulo são apresentados os resultados experimentais obtidos por meio do EACImpute, bem como sua comparação com seis algoritmos de imputação (KNNImpute (Troyanskaya et al., 2001), SKNN (Kim et al., 2004), IKNNImpute (Brás e Menezes, 2007), KMI (Hruschka et al., 2004), Média e Majority (Kononenko et al., 1984)), descritos no Capítulo 2. Os resultados obtidos em seis bases de dados de problemas de classificação foram analisados estatisticamente por meio do teste não paramétrico de Friedman (Demsar, 2006) seguido do teste post-hoc de Nemenyi (Hollander e Wolfe, 1999).

Este capítulo está organizado da seguinte forma: Na Seção 4.1 são apresentadas as principais características das bases de dados utilizadas; na Seção 4.2 são descritos os procedimentos para a simulação dos valores ausentes; na Seção 4.3 são apresentadas as metodologias para avaliação dos algoritmos tanto na predição quanto na inserção de tendências em problemas de classificação; na Seção 4.4 são apresentadas as configurações de parâmetros dos algoritmos avaliados; na Seção 4.5, são apresentados os resultados obtidos da comparação dos algoritmos; e por fim, na Seção 4.5.3 são apresentadas as considerações finais dos experimentos, juntamente com um resumo dos resultados estatísticos em cada mecanismo de simulação.

\subsection{Bases de Dados}

Os experimentos foram realizados por meio de seis bases de dados (Íris, Glass Identification, Sintética, Yeast, Segmentation, Pen-Digits), cujas principais características são apresentadas na Tabela 4.1. Todas as bases utilizadas estão associadas à tarefa de classificação e possuem somente atributos numéricos, de tal forma que se pode usar a distância Euclidiana como medida de dissimilaridade. Tais bases, exceto a Sintética, foram obtidas por meio do repositório da UCI (Asuncion e Newman, 2007), inicialmente não contendo valores ausentes. 


\begin{tabular}{|l|c|c|c|}
\hline Base de Dados & \# Instâncias & \# Atributos & \# Classes \\
\hline Íris & 150 & 4 & 3 \\
\hline Glass Identification & 214 & 9 & 6 \\
\hline Sintética & 500 & 2 & 3 \\
\hline Yeast & 1484 & 8 & 10 \\
\hline Segmentation & 2310 & 19 & 7 \\
\hline Pen-Digits & 10992 & 16 & 10 \\
\hline
\end{tabular}

Tabela 4.1: Principais características das bases de dados utilizadas.

\subsection{Simulação de Valores Ausentes}

Nesta seção são apresentados os mecanismos adotados para a simulação de valores ausentes nas bases de dados descritas na Seção 4.1. As simulações foram realizadas por meio dos mecanismos MCAR e MAR. Os valores do atributo meta (classe) de todas as instâncias foram preservados durante o processo de simulação.

\subsubsection{Mecanismo de Ausência MCAR - Completamente Aleatório}

O processo de simulação de valores ausentes baseado no mecanismo de ausência MCAR, discutido na Seção 2.1, foi realizado de forma supervisionada. Dessa forma, para cada base de dados foram separados conjuntos de dados contendo apenas instâncias de suas respectivas classes, e então foi aplicado o processo de simulação de valores ausentes - separadamente para instâncias de cada classe.

No mecanismo de omissão de valores MCAR, o processo consiste em selecionar, aleatoriamente, instâncias que conterão códigos de ausência em seus atributos, conforme apresentado no Capítulo 2. O procedimento de simulação de valores ausentes MCAR foi realizado nas proporções de 10\%, 30\%, 50\% e 70\% das instâncias da base de dados (estratificando-se por classe). Para cada taxa de valores ausentes, uma quantidade de atributos que possuirão valores ausentes, $q_{a}$, foi variada arbitrariamente de 1 até $\frac{m}{2}$, sendo $m$ a quantidade de atributos da base de dados. Em seguida, para cada instância selecionada e para cada taxa de ausentes, são simulados valores ausentes em $q_{a}$ atributos escolhidos aleatoriamente. Dessa forma, cada instância selecionada poderá conter diferentes atributos com valores omitidos. O procedimento de simulação de valores ausentes foi realizado 30 vezes para cada taxa de valores ausentes, conforme apresentado no Algoritmo 9.

\subsubsection{Mecanismo de Ausência MAR - Aleatório}

O mecanismo de simulação MAR, conforme discutido no Capítulo 2, consiste na omissão de valores em determinados atributos baseada nos valores conhecidos de outros atributos. Para este mecanismo três bases de dados - Íris (Seção 4.1), Segmentation (Seção 4.1) e uma base 

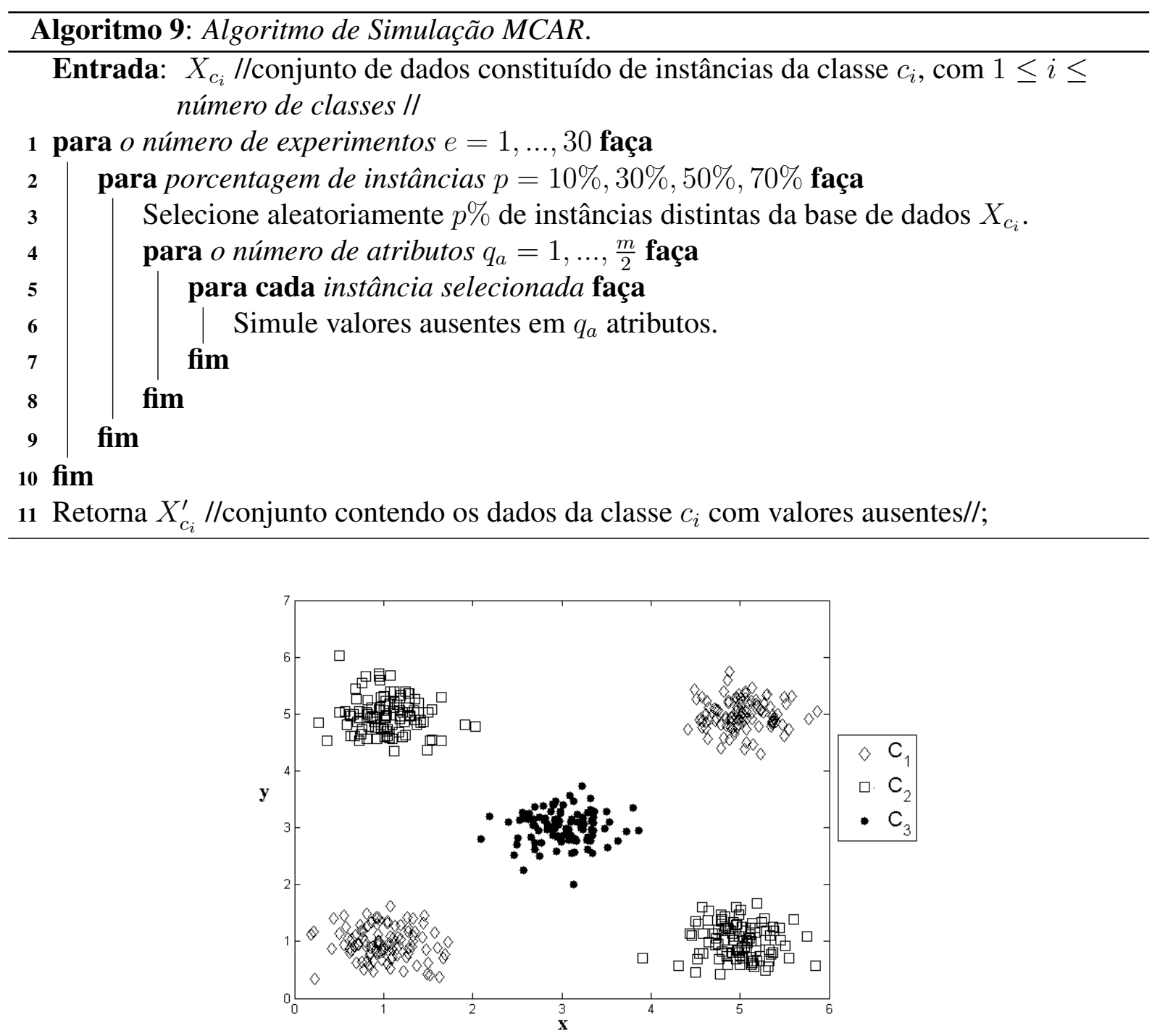

Figura 4.1: Base de dados sintética.

de dados Sintética (Garcia e Hruschka, 2005) bi-dimensional constituída de 3 classes - foram utilizadas. Esta última contém 500 instâncias separadas em 5 grupos, conforme se pode observar na Figura 4.1.

Para a simulação de valores ausentes foi aplicado inicialmente um procedimento de seleção de atributos. Este procedimento foi utilizado para simular valores ausentes, de maneira controlada, em atributos que são significativos (discriminativos) em problemas de classificação. Além disso, também é necessário ter conhecimento da faixa de valores dos atributos selecionados que auxiliam na discriminação de determinadas classes. Estes valores podem ser obtidos, por exemplo, por meio de uma árvore de decisão. Dessa maneira, são obtidos os atributos mais significativos, em problemas de classificação, e suas respectivas faixas de valores que melhor distinguem as classes. Para este mecanismo, a inserção de valores ausentes no melhor atributo selecionado é condicionada aos valores significativos de outros atributos. Resumidamente, a estratégia adotada para o mecanismo MAR se baseia em inserir valores ausentes nos atributos 
para os quais os algoritmos de aprendizagem se baseiam para a construção de um modelo de classificação. Neste mecanismo, pode-se verificar de maneira mais contundente o comportamento dos algoritmos de imputação no processo de aprendizagem do classificador.

O procedimento de simulação MAR foi realizado com o auxílio do software Weka (Garner, 1995). O procedimento de seleção de atributos foi realizado por meio de uma estratégia wrapper (Kohavi e John, 1997) utilizando o algoritmo de classificação J4.8 (baseado em árvore de decisão). Nessa estratégia, o processo de seleção consiste na busca de um subconjunto de atributos na base de dados que maximize a acurácia do classificador. Mais precisamente, o procedimento de simulação de valores ausentes MAR foi realizado da seguinte forma:

1. Selecionar atributos com a estratégia wrapper (Kohavi e John, 1997) utilizando o algoritmo de classificação J4.8, com validação cruzada em 10 pastas (amostras).

2. Ordenar cada atributo selecionado pela sua relevância, de forma que o atributo mais frequentemente selecionado em 10 pastas seja o atributo mais relevante e o segundo atributo mais frequentemente selecionado seja o segundo mais relevante, e assim por diante.

3. Identificar, para cada atributo selecionado, uma faixa de valores característicos para cada classe, por meio da árvore de decisão J4.8 construída com o conjunto de dados contendo os atributos selecionados, exceto o melhor atributo (que será usado para simular os valores ausentes). Esta etapa é realizada percorrendo os nós da árvore de decisão a partir de um nó raiz até um nó folha.

4. Selecionar aleatoriamente $10 \%, 30 \%, 50 \%$ e $70 \%$ de instâncias da base de dados que pertencem a faixa (intervalo) de valores definida para os atributos selecionados (exceto para o melhor atributo, que será usado para a simulação de valores ausentes). Em seguida, simular os valores ausentes em cada percentual de ausentes. De maneira mais formal, este passo pode ser descrito por meio de probabilidades como: $p\left(a_{1}=? \mid a_{r} \in F_{a_{r}}\right)=p_{i}$, sendo que $p_{i} \in\{0.1,0.3,0.5$ e 0.7$\}$ e $i=1, \ldots, 4$, no qual o valor ausente está representado pelo símbolo "?", $r$ representa a posição do atributo na ordem de relevância $(2 \leq r \leq s)$ e $s<m$ representa o número de atributos selecionados ( $a_{1}$ é o melhor atributo) e $F_{a_{r}}$ é a faixa de valores do atributo $a_{r}$, identificada no Passo 3 . Os valores $0.1,0.3,0.5$ e 0.7 representam as taxas de valores ausentes, respectivamente $10 \%, 30 \%, 50 \%$ e $70 \%$.

\section{Realizar 30 execuções do Passo 4.}

Para a simulação de valores ausentes na base de dados Sintética, não foi necessário aplicar a seleção de atributos nem gerar a árvore de decisão para verificar a faixa de valores, dado que a distinção entre classes pode ser obtida via inspeção visual. Nesta base de dados, a faixa de valores determinada visualmente foi $F_{x}=[0,2]$ e arbitrariamente determinado o atributo $y$ para conter valores ausentes. De maneira formal, a probabilidade $p(y=? \mid x \in[0,2])=p_{i}$, na qual $p_{i} \in\{0.1,0.3,0.5,0.7\}$ e $i=1, \ldots, 4$. Conforme se pode verificar na Figura 4.2, duas classes 


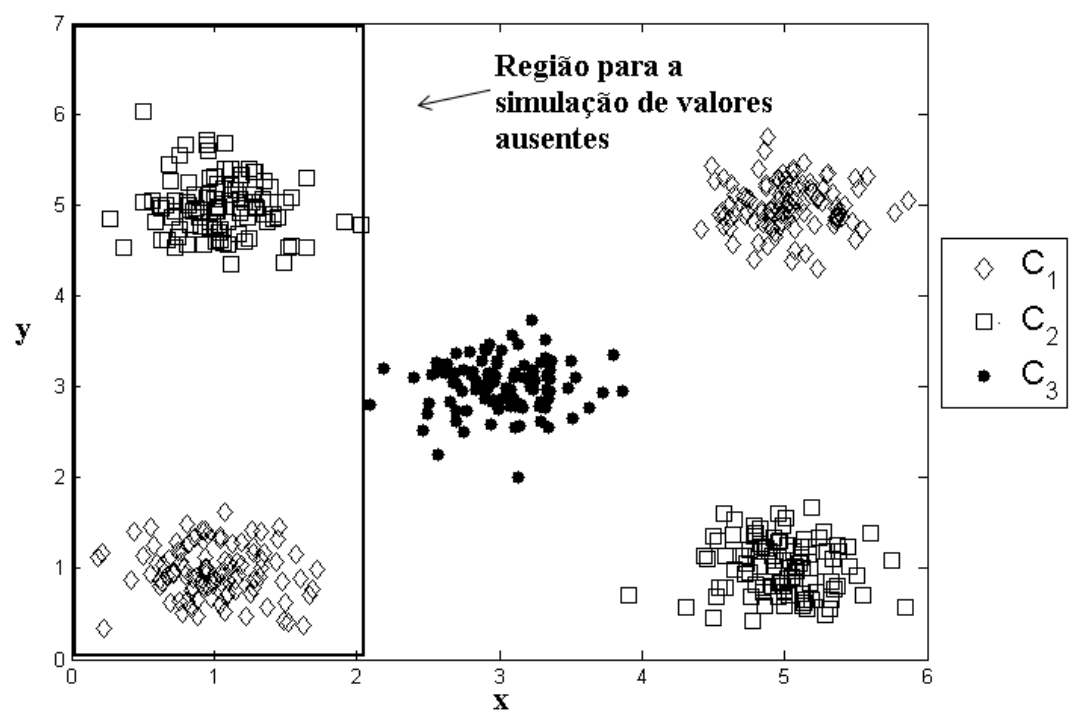

Figura 4.2: Base de dados sintética com valores ausentes simulados por meio do mecanismo MAR com probabilidade $p(y=? \mid x \in[0,2])=0,5$. O quadrado ilustra os pontos (instâncias) que conterão valores ausentes.

terão instâncias com valores ausentes (neste caso, a classe $C_{1}$ e a classe $C_{2}$ ). As simulações de valores ausentes no atributo $y$ foram realizadas apenas nas instâncias em que os valores do atributo $x$ pertencem a faixa de valores $F_{x}=[0,2]$. O efeito de simular os valores ausentes no atributo $y$, considerando a faixa $F_{x}=[3.86,6]$, é equivalente ao procedimento adotado. A faixa de valores $[2,4]$ não foi adotada por conter somente instâncias de uma única classe.

Para a base de dados Íris, após a etapa de seleção de atributos, foram obtidos os atributos Largura de Pétala (LP) e Comprimento de Pétala (CP), respectivamente o melhor atributo e o segundo melhor atributo. Dessa forma, o atributo que conterá valores ausentes é o atributo LP. Na Figura 4.3 é apresentada a base de dados Íris visualizada em 2 dimensões por meio dos atributos LP e CP. Com base nesta figura, simulou-se valores ausentes de três formas para avaliar possíveis condições em que a inserção de valores ausentes poderá influenciar de maneira significativa o processo de classificação. A primeira forma considerou todas as instâncias pertencentes à faixa $F_{C P}=[3,7]$. A segunda forma considerou a faixa $F_{C P}=[4.5,5.1]$. E, por fim, na terceira forma foram consideradas, concomitantemente, as faixas $F_{C P}=[3,4.5] \mathrm{e}$ $F_{C P}=[5.1,7]$. Na Figura 4.4 são apresentadas as 3 formas de simulação de valores ausentes.

Por fim, na base de dados Segmentation, o passo de seleção de atributos com a estratégia wrapper sugeriu o uso dos seguintes atributos: $a_{19}$ (melhor), $a_{11}, a_{2}$ e $a_{1}$ (quarto melhor). Em seguida, foi induzida a árvore de decisão usando-se apenas os atributos $a_{11}, a_{2}$ e $a_{1}$, com o objetivo de se estudar faixas de valores potencialmente interessantes para inserir valores ausentes em $a_{19}$. Para encontrar tais faixas de valores, primeiro foram identificados quais nós folhas da árvore representavam o maior número de instâncias. Em seguida, foram extraídas regras obtidas por meio dos caminhos do nó raiz da árvore até os nós folhas identificados (neste caso, foram identificados cinco nós folhas). Estas regras compreendem restrições referentes aos valores dos 


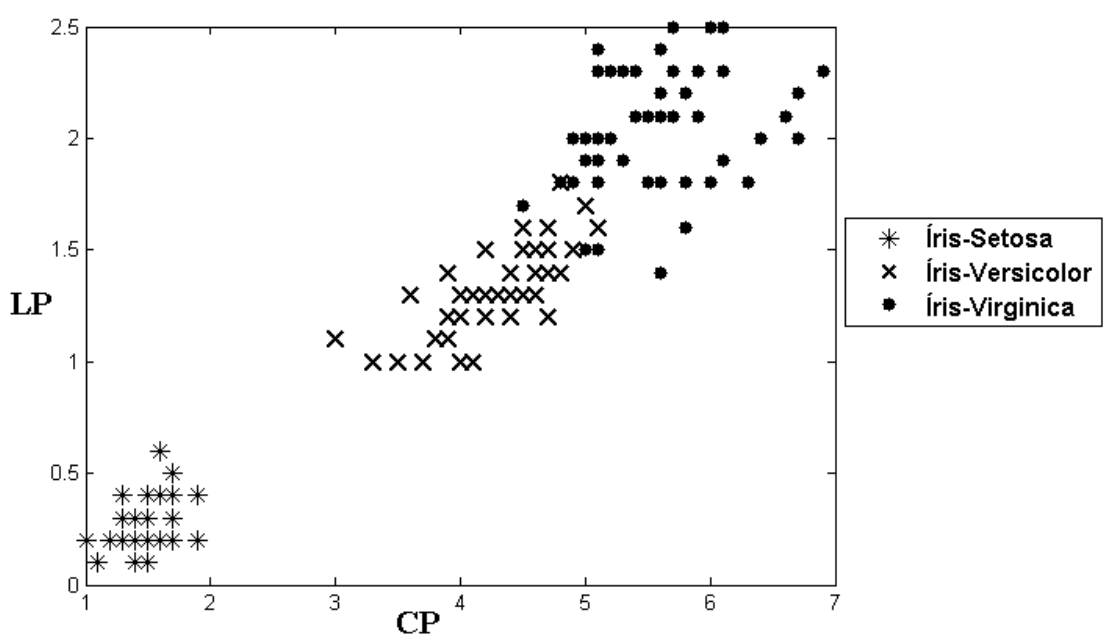

Figura 4.3: Base de dados Íris, visualizada por meio dos 2 melhores atributos (LP e CP).

atributos $a_{11}, a_{2}$ e $a_{1}$ que serão utilizadas para simular os valores ausentes no atributo $a_{19}$. 


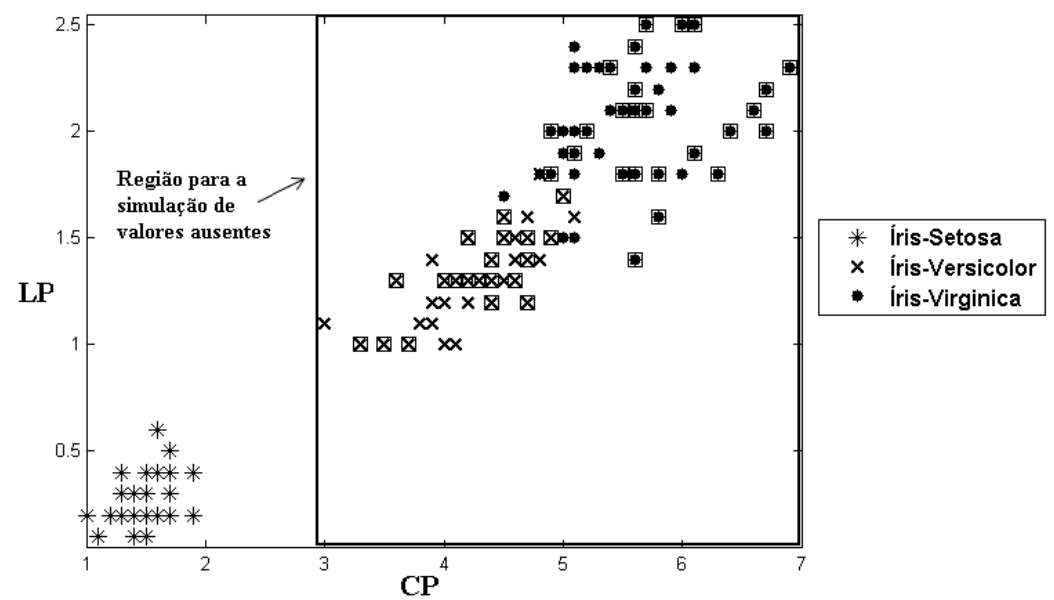

(a) Valores ausentes simulados nas instâncias da base de dados Íris com probabilidade $p(L P=$ ? $\mid 3 \leq C P \leq 7)=0.5$.

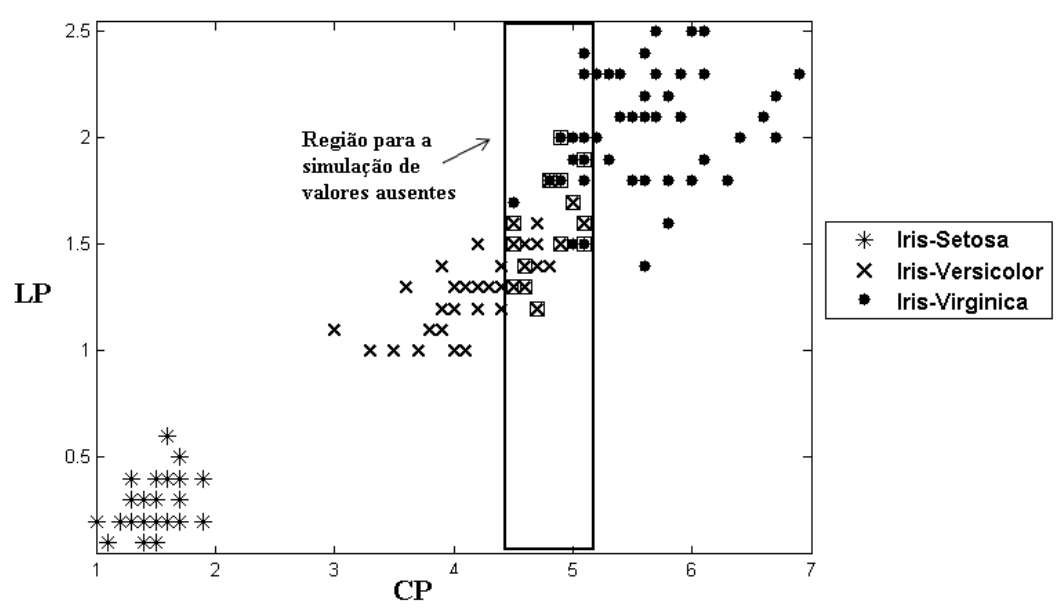

(b) Valores ausentes simulados nas instâncias da base de dados Íris com probabilidade $p(L P=? \mid 4.5 \leq C P \leq 5.1)=0.5$.

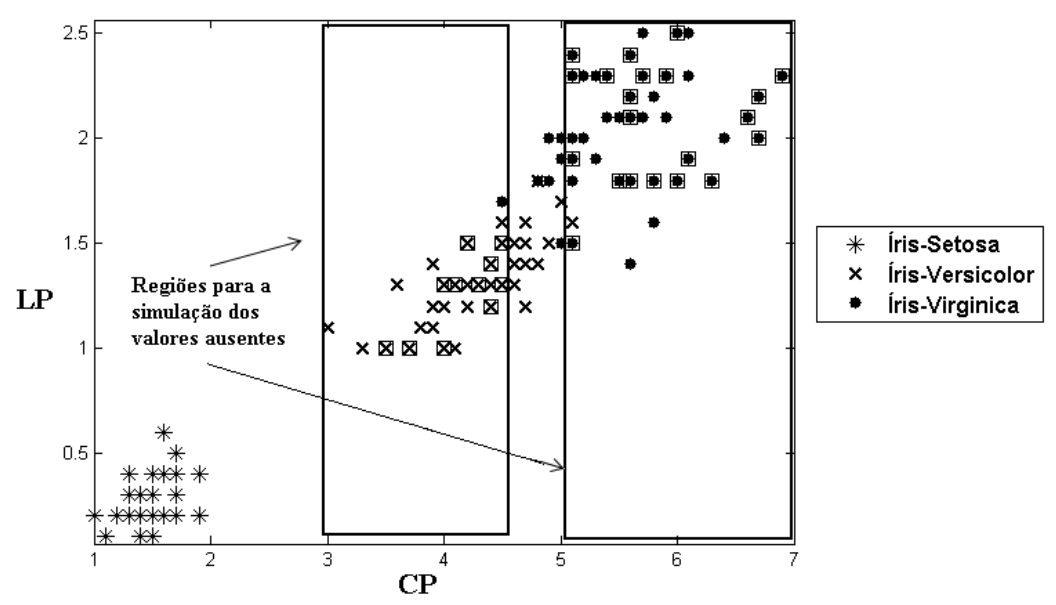

(c) Valores ausentes simulados nas instâncias da base de dados Íris com probabilidade $p(L P=? \mid 3 \leq C P \leq 4.5 \vee 5.1 \leq C P \leq 7)=0.5$.

Figura 4.4: Simulação de valores ausentes no mecanismo MAR para a base de dados Íris. Na Figura (a),(b) e (c) são apresentadas respectivamente exemplos para a primeira, segunda e a terceira forma de simulação de valores ausentes. Cada quadrado, envolvendo os símbolos " $\times$ " e “•”, indica que a instância contém valores ausentes no atributo LP. 


\subsection{Metodologia}

Dois métodos têm sido utilizados na literatura para avaliar a qualidade das imputações. Estes métodos serão aqui referenciados como métodos de predição e de modelagem. O primeiro método, amplamente utilizado na literatura, se baseia na raiz quadrada do erro quadrático médio normalizado pelo desvio padrão, aqui denominado como $\overline{E Q_{N}}$ (Brás e Menezes, 2007; Tuikkala et al., 2008), enquanto que o segundo método se destina a estimar a influência da imputação na tarefa de classificação (Hruschka et al., 2009b).

\subsubsection{Predição}

A capacidade de predição dos métodos de imputação é avaliada por meio da $\overline{E Q_{N}}$, conforme apresentado na Equação (4.1), sendo que $x_{P l}^{i} \in X_{P}$ (matriz contendo valores imputados) e $x_{O l}^{i} \in X_{O}$ (matriz contendo valores originais), $N_{P}$ é a quantidade de valores imputados em $X_{P}$ e $\sigma_{X_{O}}$ é o desvio padrão para os valores originais correspondentes a todas as instâncias que contêm valores imputados.

$$
\overline{E Q_{N}}=\frac{1}{\sigma_{X_{O}}} \sqrt{\frac{\sum_{i=1}^{N} \sum_{l=1}^{m}\left(x_{P l}^{i}-x_{O l}^{i}\right)^{2}}{N_{P}}}
$$

Esta metodologia de avaliação é muito utilizada na literatura (Oba et al., 2003; Kim et al., 2005; Brás e Menezes, 2007) para avaliar algoritmos mais adequados para imputação. O princípio desta metodologia é avaliar o quão similar um valor imputado é em relação ao valor original. Quanto mais próximo de zero é o valor do erro, melhor é o algoritmo de imputação.

\subsubsection{Classificação}

\subsubsection{Imputação em problemas de classificação}

Em problemas de classificação, cada instância $\mathbf{x}^{i}$ do conjunto de dados, $\mathbf{x}^{i}=\left[x_{1}^{i}, x_{2}^{i}, \ldots, x_{m}^{i}\right]$, contendo $m$ atributos $a_{l}(l=1, \ldots, m)$, corresponde a uma classe $c_{i} \in C=\left\{c_{1}, \ldots, c_{i}, \ldots, c_{p}\right\}$, $\operatorname{com} p$ representando o número de classes. As informações das classes são úteis para o processo de imputação (Delavallade e Dang, 2007). Neste sentido, o processo de imputação pode ser adaptado para levar em conta apenas instâncias da mesma classe. Esta estratégia é chamada de imputação supervisionada (Garcia e Hruschka, 2005). Para exemplificar o processo de imputação de maneira supervisionada, considere uma base de dados $X_{D}$, na forma matricial, contendo um conjunto de instâncias $X_{D}=\left\{\mathbf{x}^{1}, \ldots, \mathbf{x}^{i}, \ldots, \mathbf{x}^{15}\right\}$ (neste caso, com $N=15$ ) contendo 4 atributos $a_{l}(l=1,2,3,4)$. Cada instância está associada a uma classe $c_{i}$ de um conjunto de classes $C=\left\{c_{1}, c_{2}, c_{3}\right\}$ - conforme apresentado na Figura 4.5. O conjunto de instâncias $X_{D}=X_{C} \cup X_{A}$ é constituído de um conjunto de instâncias com valores completos, $X_{C}$, e um conjunto no qual cada instância contém pelo menos um valor ausente, $X_{A}$. Neste exemplo, 


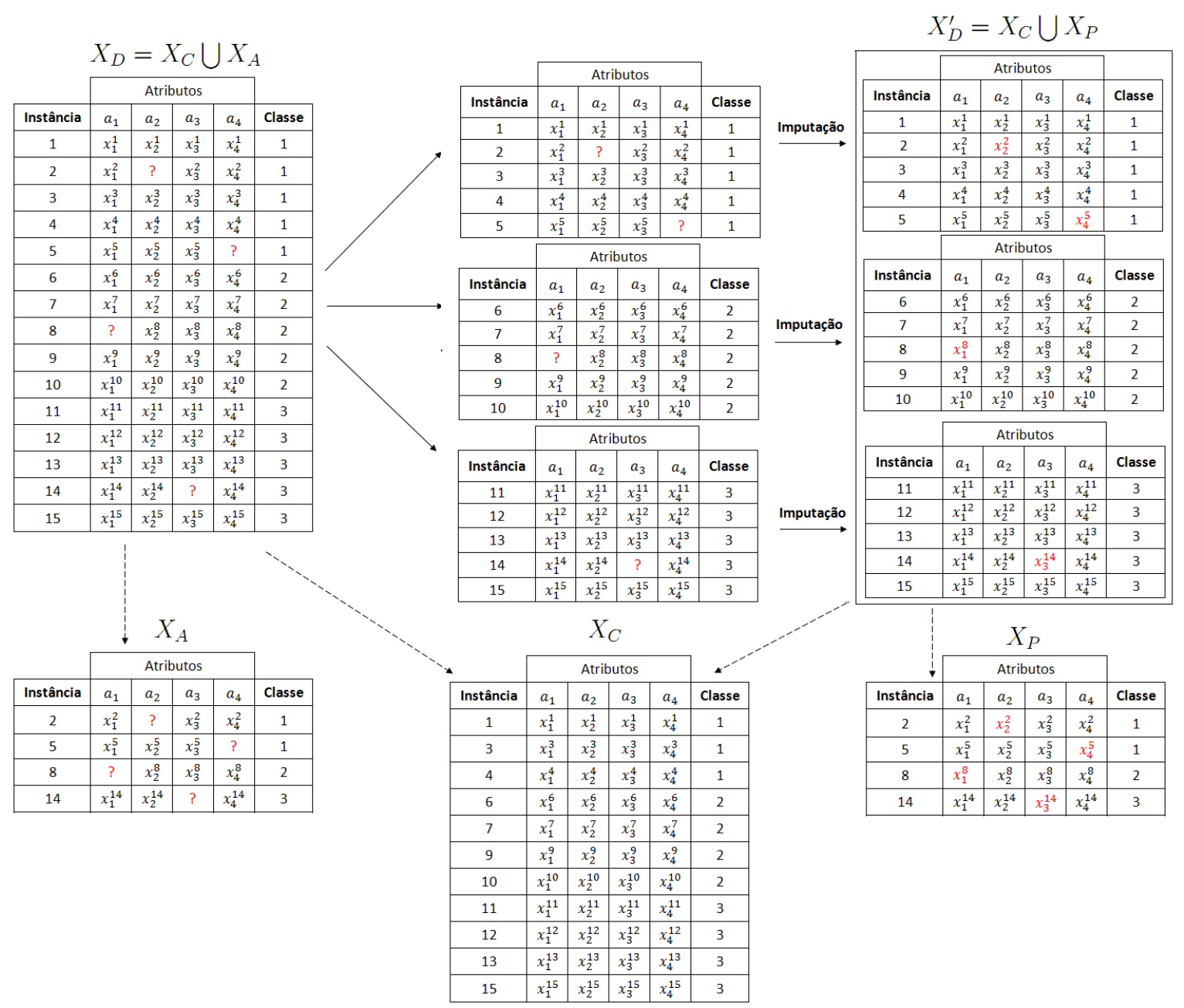

Figura 4.5: Exemplo ilustrativo do processo de imputação de maneira supervisionada.

o conjunto de dados $X_{D}$ foi dividido em três subconjuntos (pois há 3 classes) nos quais são aplicados ao processo de imputação separadamente. Após o processo de imputação, obtém-se o conjunto de dados $X_{D}^{\prime}=X_{C} \bigcup X_{P}$ compreendido das instâncias com valores completos, $X_{C}$, e preenchidos, $X_{P}$. As linhas tracejadas ligadas às matrizes $X_{A}, X_{C}$ e $X_{P}$ apenas ilustram a decomposição das matrizes $X_{D}$ e $X_{D}^{\prime}$.

\subsubsection{Estimação do viés na classificação}

Seguindo o raciocínio desenvolvido em (Hruschka et al., 2009b), uma situação ideal para o método de imputação é preencher os valores ausentes originando valores imputados sem inserir nenhum viés no conjunto de dados. Em uma visão mais realista, os métodos de imputação, supostamente, deveriam minimizar a quantidade de tendências inseridas a níveis aceitáveis, de forma que o conjunto de dados $X_{D}^{\prime}=X_{C} \bigcup X_{P}$ (Figura 4.5), provavelmente contendo mais informações do que $X_{C}$, possa ser utilizado para mineração de dados (e.g., considerando questões relacionadas à seleção de atributos, combinação de múltiplos modelos e assim por diante). Nesse sentido é particularmente importante enfatizar que se assumirá que os valores 
conhecidos em $X_{A}$ podem conter informações importantes para o processo de modelagem. Estas informações seriam parcialmente perdidas se as instâncias e/ou atributos com valores ausentes fossem ignorados.

Conforme mencionado na Seção 4.3.1, métodos amplamente utilizados na literatura para avaliar imputações consistem na predição e, mais recentemente a influência das imputações na modelagem tem sido investigada. No método de predição, os valores ausentes são simulados, i.e., alguns valores conhecidos são removidos e então imputados. Por exemplo, alguns valores conhecidos de $X_{C}$ podem ser artificialmente eliminados, simulando dados ausentes. Deste modo é possível avaliar o quão similar os valores imputados são dos valores reais, conhecidos $a$ priori. A suposição por trás deste método é que quanto mais os valores imputados são similares aos valores reais, melhor é o algoritmo de imputação. Embora a predição é um método válido e amplamente adotado na literatura, os resultados da predição não são os mais importantes a serem analisados, conforme discutido por exemplo em (Hruschka et al., 2009b). Resumidamente, a predição não permite estimar o viés sob a perspectiva de modelagem. Mais precisamente, o processo de substituição deve gerar valores com mínima distorção das características originais de $X_{D}$, as quais podem ser assumidas como os relacionamentos entre os atributos para processos de modelagem. Estes relacionamentos, frequentemente explorados por algoritmos de classificação, expressam o modo como as mudanças dos valores de um atributo modificam também os valores de outros atributos (Pyle, 1999).

Uma difícil tarefa para os algoritmos de imputação consiste em preservar os relacionamentos entre os atributos - na medida do possível. Dessa forma, utilizar valores inapropriados estimados pelos algoritmos de imputação pode distorcer as características existentes nos dados e introduzir padrões espúrios, aqui denominados "viés" - bias (Pyle, 1999). Para facilitar o entendimento do problema, considere o exemplo pedagógico apresentado na Figura 4.6. Este exemplo é inspirado no bem conhecido conjunto de dados Íris, o qual é formado por quatro atributos (Comprimento da Sépala-CS, Largura da Sépala-LS, Comprimento da Pétala-CP e Largura da Pétala-LP) e o rótulo da classe $c_{i} \in C=\{$ Íris-setosa, Iris-versicolor, Íris-virgínica $\}$. Considere que a instância cujo ID é 151 tem um valor ausente no atributo LP. Esta instância é idêntica à instância 44, exceto pelo valor ausente (“?”). Em outras palavras, a instância 151 poderia ser vista como o resultado de um procedimento para simulação de valores ausentes amplamente utilizado na literatura. Neste contexto, um método de imputação deveria estimar um valor tão próximo quanto possível ao valor 0,6 , que é o valor conhecido e que foi artificialmente excluído da instância 44. Agora assuma que dois métodos de imputação (A e B) estão disponíveis para substituir tais valores ausentes. Além disso, considere que o método de imputação A substitui o valor ausente por 0,2 e que o método B substitui o valor ausente por 0,601. Claramente o método B é melhor do que o método A do ponto de vista da predição. Entretanto, considere agora que a árvore apresentada na Figura 4.6 é um classificador perfeito. De acordo com este classificador, o método de imputação B faria com que a instância 151 seja incorretamente classificada, sendo que o método de imputação A, que não é tão bom quanto B 


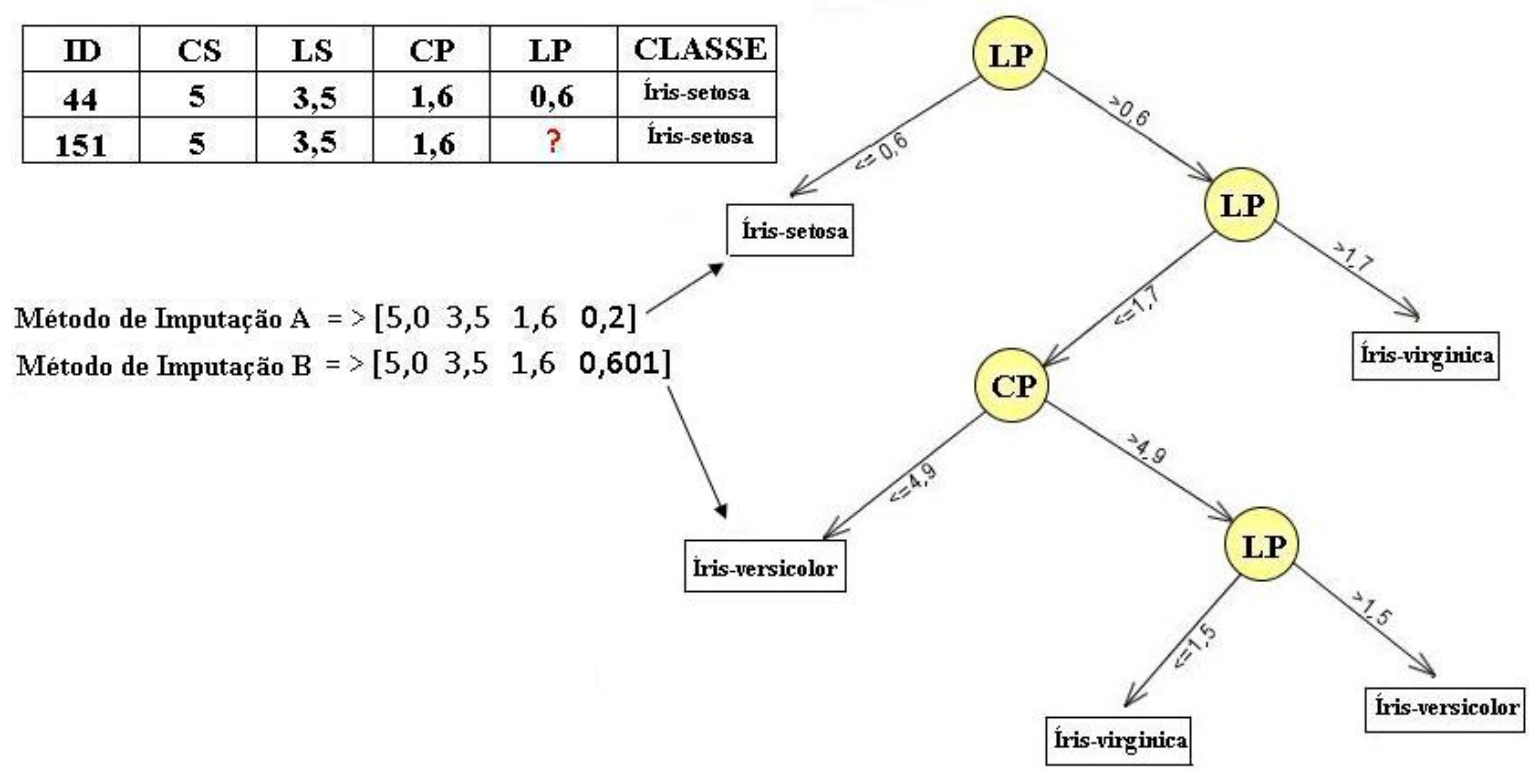

Figura 4.6: Exemplo ilustrativo do viés de classificação.

do ponto de vista da predição, levaria a uma correta classificação da instância considerada. Este exemplo ilustra que melhores predições não indicam, necessariamente, melhores resultados de classificação.

Vários outros autores, conforme discutido em Hruschka et al. (2009b), também argumentam que é mais importante levar em conta a influência dos valores imputados em processos de modelagem (e.g., preservar as relações entre os atributos) do que obter predições mais precisas. Pyle (1999) explica que, embora os valores imputados são predições, a precisão destas predições não é a informação mais importante a ser considerada quando valores ausentes são imputados. $\mathrm{O}$ princípio subjacente a este raciocínio é que as predições devam produzir estimativas razoáveis, com pouca distorção dos valores que estão atualmente presentes no conjunto de dados, sendo que a distorção pode se referir ao relacionamento entre os atributos. Em outras palavras, a proposta principal dos algoritmos de imputação não é utilizar os próprios valores imputados, mas tornar disponível para ferramentas de modelagem às informações contidas nos valores dos outros atributos, presentes originalmente na base de dados. Por todas essas razões, neste trabalho será também estimado o viés inserido em termos dos resultados de classificação, que de alguma forma permite avaliar de que maneira a extensão dos relacionamentos entre os atributos serão mantidos após a imputação. Finalmente, há que se reconhecer que, em aplicações reais, os valores imputados não podem ser comparados com nenhum valor.

$\mathrm{O}$ viés inserido pode ser definido como a magnitude das mudanças nos relacionamentos entre os atributos, causadas por padrões introduzidos por um processo de imputação (Hruschka et al., 2009b). O problema é que os relacionamentos entre os atributos são dificilmente conhecidos de antemão (antes da etapa de mineração de dados). Por esta razão, geralmente o viés inserido não pode ser diretamente mensurado, mas pode ser estimado. A principal suposição, em problemas de classificação, é que os relacionamentos entre os atributos são induzidos por um 
1. Avaliar o classificador pela TCCM com validação cruzada em 10 pastas no conjunto de treinamento $X_{C}$, obtendo $T C C M_{C}$.

2. Avaliar o classificador - construído com $X_{C}$ (conjunto de treinamento) pela TCCM no conjunto de teste $X_{P}$.

3. O viés $(b)$ inserido pela execução da imputação é estimado pela diferença entre os resultados alcançados nos passos 2. e 1. : $\widehat{b}=T C C M_{P}-T C C M_{C}$.

Figura 4.7: Procedimento para estimar o viés na etapa de classificação.

classificador em específico. Consequentemente, a qualidade de tais relações recém descobertas pode ser indiretamente estimada por uma medida de classificação como a Taxa de Classificação Correta Média (TCCM). Nesse sentido, a metodologia proposta em Hruschka et al. (2009b) é adotada para estimação do viés inserido.

Tal metodologia se baseia no fato que, em aplicações de mineração de dados, diferentes classificadores são avaliados frequentemente para um determinado conjunto de dados, de modo que o melhor classificador disponível é escolhido de acordo com algum critério de qualidade do modelo (e.g., TCCM). A suposição é que o melhor classificador (MC) - em relação à $X_{C}$ e aos classificadores disponíveis - provê um modelo adequado para classificar instâncias após serem realizadas as imputações. Desse modo, avaliar se os valores imputados se ajustam ao MC é importante. Uma prática comum é avaliar o desempenho de um classificador em um conjunto de teste. O mesmo conceito é adaptado para avaliar as imputações, considerando $X_{C}$ como conjunto de treinamento e $X_{P}$ como conjunto de teste. Então, o viés inserido pode ser estimado por meio do procedimento apresentado na Figura 4.7. De acordo com este procedimento, um viés positivo é alcançado quando a TCCM em $X_{P}$ (passo 2) é maior do que no processo de validação cruzada em $X_{C}$ (passo 1). Neste caso, os valores imputados tendem a melhorar a TCCM do classificador em $X_{D}^{\prime}$. Consequentemente, um viés negativo é inserido quando os valores imputados provavelmente piorarão a TCCM do classificador em $X_{D}^{\prime}$. Finalmente, nenhum viés é provavelmente inserido quando a acurácia em $X_{P}$ e no processo de validação cruzada em $X_{C}$ são iguais. Assumindo que o processo de imputação não deve introduzir nenhum padrão artificial nos dados, essa é a situação ideal. De fato, estes padrões artificiais, não contidos nos valores conhecidos, podem ser posteriormente descobertos em um processo de mineração de dados em $X_{D}^{\prime}$. Entretanto, a inclusão de tais padrões artificiais, que são simplesmente um artefato do processo de imputação, deve ser evitada. Nesta metodologia, não apenas o viés negativo deve ser evitado, mas também nenhum viés positivo é desejável, pois ambos implicam que padrões artificiais foram provavelmente incorporados ao conjunto de dados (Hruschka et al., 2009b).

\subsubsection{Aplicação da Metodologia de Estimação do Viés na Classificação}

Para a execução dos experimentos em problemas de classificação foram selecionados, para cada base de dados, os classificadores que obtiveram o melhor desempenho na classificação das instâncias. Este procedimento foi realizado com quatro classificadores bem conhecidos 


\begin{tabular}{|l|c|c|c|c|}
\hline Base $^{\text {Classificador }}$ & $\mathbf{J 4 . 8}$ & $\boldsymbol{k}$-NN $(\boldsymbol{k = 3})$ & MLP & NB \\
\hline Íris & $94,7 \%$ & $95,2 \%$ & $\mathbf{9 6 , 9 \%}$ & $95,5 \%$ \\
\hline Glass Identification & $67,6 \%$ & $\mathbf{7 0 \%}$ & $67,3 \%$ & $49,4 \%$ \\
\hline Yeast & $56,4 \%$ & $54,3 \%$ & $\mathbf{5 8 , 8 \%}$ & $57,9 \%$ \\
\hline Segmentation & $96,4 \%$ & $\mathbf{9 9 , 3 \%}$ & $94,6 \%$ & $85,8 \%$ \\
\hline Pen-Digits & $\mathbf{9 6 , 8 \%}$ & $96,1 \%$ & $96,0 \%$ & $80,3 \%$ \\
\hline
\end{tabular}

Tabela 4.2: Resultados de acurácia para os classificadores J4.8, $k$-NN, MLP e NB. Em negrito estão destacados as melhores taxas de classificação corretas médias (TCCM) para cada base de dados.

disponíveis no software $\mathrm{Weka}^{1}$ : Árvore de decisão (J4.8), $k$-NN, Multilayer Perceptron (MLP) e Nä̈ve Bayes (NB), conforme apresentados na Tabela 4.2.

Por meio dos resultados obtidos na Tabela 4.2, o classificador J4.8 foi então definido para a base de dados Pen-Digits, o classificador $k$-NN para as bases Glass Identification e Segmentation, e finalmente para as bases Íris e Yeast o classificador MLP foi adotado. Os experimentos foram realizados utilizando parâmetros default dos algoritmos de classificação disponíveis no Weka. Esses classificadores foram definidos para a execução dos experimentos no mecanismo MCAR. Para o mecanismo MAR, o classificador J4.8 foi utilizado, o qual foi adotado na seleção de atributos, para as bases de dados Sintética (não apresentada na Tabela 4.2), Íris e Segmentation.

\subsection{Configuração de Parâmetros para os Algoritmos de Imputação}

A execução dos algoritmos de imputação (apresentados no Capítulo 2) foi realizada com a determinação dos parâmetros propostos pelos seus próprios autores. Nos algoritmos KNNimpute (Troyanskaya et al., 2001), SKNN (Kim et al., 2004) e IKNNimpute (Brás e Menezes, 2007) foi utilizado o valor 10 para o parâmetro referente ao número de vizinhos mais próximos, seguindo resultados de experimentos realizados em Troyanskaya et al. (2001). Para o algoritmo IKNNimpute (Brás e Menezes, 2007), o parâmetro referente ao número de ciclos foi definido igual a 2 e o limiar $\tau$ para o critério de convergência igual a 0,001, conforme definido em Brás e Menezes (2007). No algoritmo KMI (Hruschka et al., 2004) foi definido o número de grupos em $2(K=2)$, conforme reportado em Hruschka et al. (2004).

No algoritmo de imputação EACImpute não é necessário definir a priori o número de vizinhos (parâmetro $k$ ). Esse parâmetro é definido automaticamente pela cardinalidade de cada grupo de instâncias. Nesse algoritmo, a função de distância Euclidiana normalizada adotada pelos algoritmos descritos no Capítulo 2 também foi utilizada. Em relação ao número de gerações e o tamanho da população, estes foram definidos arbitrariamente em 20 e 5 respectivamente. $\mathrm{O}$

\footnotetext{
${ }^{1}$ disponível em http://www.cs.waikato.ac.nz/ml/weka
} 
número de gerações para se atingir a convergência e o tamanho da população são inversamente proporcionais, de forma que na medida que o tamanho da população aumenta menos gerações são necessárias para que a convergência seja alcançada. Para a realização do procedimento de busca local, com o algoritmo das $K$-médias, foram definidos dois critérios de parada: i) número máximo de iterações igual a 5; ii) diferença entre centróides, em duas iterações consecutivas, menor do que 0,001. Quando um dos critérios é alcançado o algoritmo encerra a sua execução.

Em cada cenário de valores ausentes foram realizadas avaliações comparativas do EACImpute com os algoritmos KNNimpute, SKNN, IKNNimpute, KMI e os métodos de imputação da média condicionada e não condicionada à informação da classe. Estas comparações foram realizadas tanto na predição quanto na classificação. Os métodos descritos foram implementados na linguagem de programação Java 1.5 .

\subsection{Resultados Obtidos}

Nesta seção são apresentados os resultados obtidos sob o ponto de vista da predição e da classificação, conforme discutido na Seção 4.3 em simulações de valores ausentes realizadas com os mecanismo MCAR e MAR. Análises estatísticas foram realizadas para cada medida de avaliação ( $\overline{E Q_{N}}$ e o viés de classificação) em cada cenário de ausência. Um cenário de ausência é constituído pela combinação entre a taxa de valores ausentes e o número de atributos com valores ausentes. Para cada cenário de ausência, resultados de 30 experimentos (repetições) foram avaliados estatisticamente. Os resultados de cada repetição executada, considerando os 7 algoritmos avaliados, foram dispostos em uma sequência de classificação ("rank"), de forma que para o melhor valor (menor erro de predição ou viés de classificação) foi atribuído o valor 1 e para o pior foi atribuído o valor 7. Em caso de empates, a classificação é determinada pela média dos "ranks" atribuídos. Baseando-se nesses resultados, foi aplicado o teste de Friedman (Demsar, 2006) que testa a veracidade da hipótese nula, afirmando que todos os algoritmos apresentam o mesmo desempenho. De maneira análoga, uma hipótese alternativa afirma que os algoritmos apresentam desempenhos diferentes. O teste de Friedman verifica quando as médias dos "ranks" - neste caso, para 30 repetições - são significativamente diferentes em relação aos valores médios dos "ranks" dos algoritmos, supondo que estes apresentariam o mesmo desempenho (hipótese nula). Em seguida, caso a hipótese nula seja rejeitada, procede-se então com o teste post-hoc de Nemenyi (Hollander e Wolfe, 1999) para verificar quais pares de algoritmos obtiveram diferença estatística. Os testes estatísticos foram realizados com $\alpha=0,05$. A seguir são apresentados os resultados obtidos em cada base de dados. Os resultados estatísticos foram obtidos por meio de um algoritmo de análise estatística para múltiplas comparações ${ }^{2}$ proposto em (García e Herrera, 2008).

\footnotetext{
${ }^{2}$ Código fonte implementado em Java disponível em: http://sci2s.ugr.es/keel/multipleTest.zip
} 


\subsubsection{Completamente Aleatório - MCAR}

\subsubsection{Base de dados "Íris"}

Os resultados para a base de dados Íris referentes aos valores médios da predição com a medida de avaliação $\overline{E Q_{N}}$ - Equação 4.1, para as taxas de $10 \%, 30 \%$, 50\% e $70 \%$ de valores ausentes, são apresentados na Figura 4.8. Para efeito de melhor visualização, dois gráficos Figuras 4.8(a) e 4.8(b) são apresentados. O gráfico da Figura 4.8(a) apresenta os valores médios do erro $\overline{E Q_{N}}$ para todos os algoritmos avaliados. Nesta figura, verifica-se uma diferença visível do algoritmo da média não condicionada à classe, aqui denominado de Média Geral, para os demais algoritmos. O gráfico da Figura 4.8(b) apresenta os resultados comparativos entre todos os algoritmos, exceto para o algoritmo Média Geral (que apresentou piores resultados). Nestes resultados, o algoritmo IKNNImpute apresentou melhores resultados (em termos de predição) para todas as taxas de valores ausentes.

A Tabela 4.3 apresenta os resultados referentes aos valores médios dos "ranks" de cada algoritmo (para 30 experimentos executados em cada cenário de ausência). Menores valores de "rank" indicam melhores algoritmos. Neste caso, o algoritmo IKNNImpute obteve os melhores resultados em todos cenários de ausência. Por meio dos resultados apresentados na Tabela 4.3, comparações pareadas dos algoritmos foram realizadas, nos quais mostram o número de vezes que os valores médios dos "ranks" foram inferiores, iguais ou superiores comparativamente a algum algoritmo usado neste estudo. Resultados dessas comparações pareadas podem ser encontrados na Tabela A.1 do Apêndice. Para uma melhor compreensão destes resultados, considere o exemplo da comparação entre o algoritmo Média Geral (primeira linha com valores da tabela) e o algoritmo Média Majority (quarta coluna da tabela) com valores ausentes em 10\% das instâncias. Observando apenas os resultados obtidos por esses dois algoritmos na Tabela A.1 do Apêndice, percebe-se que o algoritmo Média Geral perdeu 2 vezes para o algoritmo Média Majority. Estas derrotas se dão pelo fato de que os valores médios dos "ranks" do algoritmo Média Geral foram superiores aos do algoritmo Média Majority nos cenários com 1 e 2 atributos com valores omitidos na taxa de $10 \%$ valores ausentes, conforme se pode verificar nas colunas 3 e 4 da Tabela 4.3.

Após a realização das comparações pareadas, a Tabela 4.4 apresenta os resultados obtidos pelo somatório do número de vitórias, empates e derrotas de cada algoritmo listado na Tabela A.1 do Apêndice. Dos resultados da Tabela 4.4, vale ressaltar que o algoritmo IKNNImpute apresentou maior número de vitórias, somando 12 vitórias, em relação aos algoritmos comparados para cada taxa de valores ausentes.

Diferenças estatísticas em relação aos valores médios dos "ranks" dos algoritmos avaliados foram identificadas, por meio do teste de Friedman, para alguns cenários de ausência. Nesses cenários, procedeu-se com o teste de Nemenyi, para identificar quais pares de algoritmos obtiveram diferenças estatísticas, conforme reportado na Tabela A.2 do Apêndice. Para uma melhor compreensão dos resultados considere, como exemplo, os resultados das comparações 


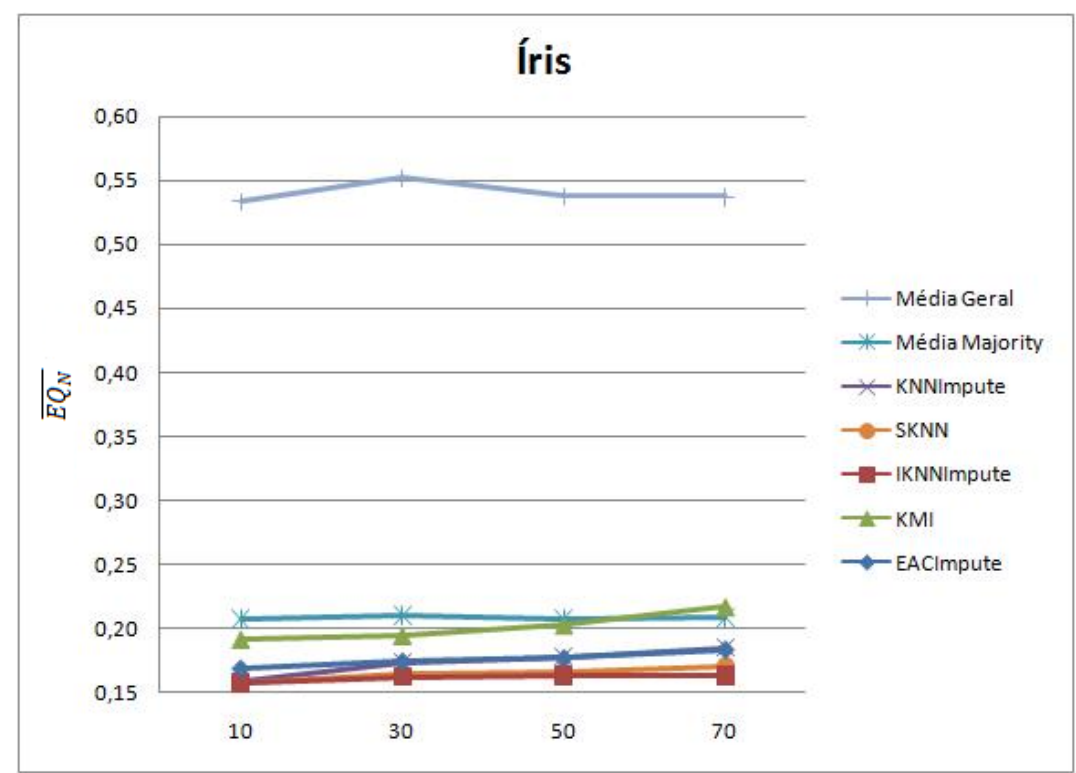

(a)

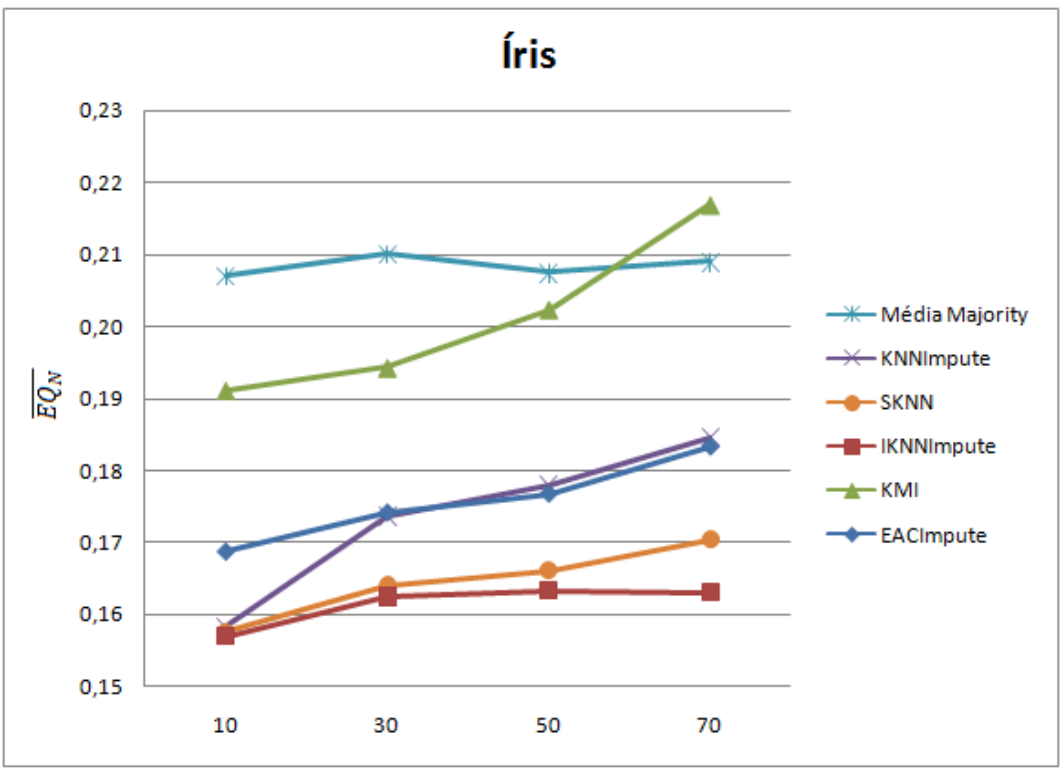

(b)

Figura 4.8: Avaliação pelo $\overline{E Q_{N}}$ para a base de dados Íris. Na Figura (a) são apresentados os resultados de todos algoritmos. Na Figura (b) são apresentados os resultados do erro desconsiderando o algoritmo Média Geral (pior algoritmo). 


\begin{tabular}{|c|c|c|c|c|c|c|c|c|}
\hline $\begin{array}{c}\text { Taxa de } \\
\text { Ausentes }\end{array}$ & $\begin{array}{l}\text { Atributos } \\
\text { Ausentes }\end{array}$ & Média Geral & Média Majority & KNNImpute & SKNN & IKNNImpute & KMI & EACImpute \\
\hline \multirow{2}{*}{$10 \%$} & 1 & 7,00 & 5,37 & 2,37 & 2,47 & 2,17 & 5,03 & 3,60 \\
\hline & 2 & 7,00 & 5,13 & 3,03 & 2,67 & 2,47 & 4,03 & 3,67 \\
\hline \multirow{2}{*}{$30 \%$} & 1 & 7,00 & 5,73 & 2,63 & 2,00 & 1,73 & 5,13 & 3,77 \\
\hline & 2 & 7,00 & 5,57 & 4,17 & 2,47 & 2,07 & 4,00 & 2,73 \\
\hline \multirow{2}{*}{$50 \%$} & 1 & 7,00 & 5,53 & 3,17 & 1,87 & 1,30 & 5,33 & 3,80 \\
\hline & 2 & 7,00 & 5,50 & 4,23 & 2,40 & 2,27 & 4,07 & 2,53 \\
\hline \multirow{2}{*}{$70 \%$} & 1 & 7,00 & 5,33 & 3,17 & 2,50 & 1,17 & 5,63 & 3,20 \\
\hline & 2 & 7,00 & 5,13 & 3,83 & 1,90 & 1,50 & 5,10 & 3,53 \\
\hline $\begin{array}{c}\text { Frequência } \\
\text { dos menores } \\
\text { "ranks" }\end{array}$ & - & 0 & 0 & 0 & 0 & 8 & 0 & 0 \\
\hline
\end{tabular}

Tabela 4.3: "Ranks" médios em cada cenário de ausência $-\overline{E Q_{N}}$ para a base de dados Íris. Os menores valores de "rank" médios estão destacados em negrito.

\begin{tabular}{|c|c|c|c|c|}
\hline Algoritmo & $10 \%$ & $30 \%$ & $50 \%$ & $70 \%$ \\
\hline Média Geral & $(0 / 0 / 12)$ & $(0 / 0 / 12)$ & $(0 / 0 / 12)$ & $(0 / 0 / 12)$ \\
\hline Média Majority & $(2 / 0 / 10)$ & $(2 / 0 / 10)$ & $(2 / 0 / 10)$ & $(3 / 0 / 9)$ \\
\hline KNNImpute & $(9 / 0 / 3)$ & $(6 / 0 / 6)$ & $(6 / 0 / 6)$ & $(7 / 0 / 5)$ \\
\hline SKNN & $(9 / 0 / 3)$ & $(10 / 0 / 2)$ & $(10 / 0 / 2)$ & $(10 / 0 / 2)$ \\
\hline IKNNImpute & $(12 / 0 / 0)$ & $(12 / 0 / 0)$ & $(12 / 0 / 0)$ & $(12 / 0 / 0)$ \\
\hline KMI & $(4 / 0 / 8)$ & $(5 / 0 / 7)$ & $(5 / 0 / 7)$ & $(3 / 0 / 9)$ \\
\hline EACImpute & $(6 / 0 / 6)$ & $(7 / 0 / 5)$ & $(7 / 0 / 5)$ & $(7 / 0 / 5)$ \\
\hline
\end{tabular}

Tabela 4.4: Total de vitórias, empates e derrotas, em relação ao "rank" médio $-\overline{E Q_{N}}$ para a base de dados Íris.

pareadas dos algoritmos Média Geral e Média Majority ( $<$ Média Geral, Média Majority $>$ ) na taxa de $10 \%$ de valores ausentes. Neste caso, o algoritmo Média Majority venceu nos cenários com $10 \%$ de valores ausentes ( 1 e 2 atributos) resultando 2 vitórias. Note que, nesta base de dados, existem 12 possíveis combinações (6 comparações pareadas nos cenários com 1 e 2 atributos com valores ausentes), para cada taxa de valores ausentes, em que um algoritmo A pode vencer, empatar ou perder relativamente a outro algoritmo B (em função de seus "ranks"). Nas combinações em que não foram observadas diferenças estatísticas conclui-se que os algoritmos empataram, visto que a hipótese nula não foi rejeitada e esta afirma que os algoritmos apresentam desempenhos iguais. Dessa forma, os resultados referentes aos números totais de vitórias, empates e derrotas dos algoritmos em questão nas combinações em que houve diferenças estatísticas são apresentados na Tabela 4.5. Este resultados foram obtidos pelo somatório do número de vitórias, empates e derrotas - apresentados na Tabela A.2 do Apêndice- de cada algoritmo. Destes resultados, vale destacar que, para todas as taxas de valores ausentes, o algoritmo IKNNImpute supera ou iguala os demais algoritmos em relação aos totais de vitórias (empatando com o algoritmo SKNN nas taxas de 30\% e 50\% de valores ausentes).

Sob o ponto de vista da influência dos valores imputados em problemas de classificação, os resultados médios do viés na classificação obtidos pela avaliação dos algoritmos de imputação para cada taxa de valores ausentes são apresentados na Figura 4.9. Esses resultados foram obtidos com a utilização do classificador MLP na metodologia de avaliação do viés na classificação (ver Seção 4.3), o qual obteve maior valor para a taxa de classificação correta média para esta base de dados, conforme apresentado na Tabela 4.2. A Figura 4.9(a) apresenta os resultados 


\begin{tabular}{|c|c|c|c|c|}
\hline Algoritmo & $10 \%$ & $30 \%$ & $50 \%$ & $70 \%$ \\
\hline Média Geral & $(0 / 0 / 12)$ & $(0 / 2 / 10)$ & $(0 / 0 / 12)$ & $(0 / 1 / 11)$ \\
\hline Média Majority & $(2 / 3 / 7)$ & $(0 / 3 / 9)$ & $(2 / 3 / 7)$ & $(2 / 3 / 7)$ \\
\hline KNNImpute & $(5 / 7 / 0)$ & $(5 / 5 / 2)$ & $(4 / 3 / 5)$ & $(4 / 5 / 3)$ \\
\hline SKNN & $(5 / 7 / 0)$ & $(8 / 4 / 0)$ & $(9 / 3 / 0)$ & $(8 / 4 / 0)$ \\
\hline IKNNImpute & $(6 / 6 / 0)$ & $(8 / 4 / 0)$ & $(9 / 3 / 0)$ & $(10 / 2 / 0)$ \\
\hline KMI & $(2 / 5 / 5)$ & $(3 / 4 / 5)$ & $(2 / 3 / 7)$ & $(1 / 4 / 7)$ \\
\hline EACImpute & $(4 / 8 / 0)$ & $(4 / 6 / 2)$ & $(7 / 3 / 2)$ & $(6 / 3 / 3)$ \\
\hline
\end{tabular}

Tabela 4.5: Total de vitórias, empates e derrotas de cada algoritmo obtido dos cenários de ausência em que houve diferenças estatísticas $-\overline{E Q_{N}}$ para a base de dados Íris.

\begin{tabular}{|c|c|c|c|c|c|c|c|c|}
\hline $\begin{array}{c}\text { Taxa de } \\
\text { Ausentes }\end{array}$ & $\begin{array}{c}\text { Atributos } \\
\text { Ausentes }\end{array}$ & Média Geral & Média Majority & KNNImpute & SKNN & IKNNImpute & KMI & EACImpute \\
\hline \multirow{2}{*}{$10 \%$} & 1 & 6,30 & $\mathbf{3 , 3 8}$ & 3,47 & 3,60 & 3,73 & 3,75 & 3,77 \\
\hline \multirow{2}{*}{$30 \%$} & 2 & 7,00 & $\mathbf{3 , 1 0}$ & 3,25 & 3,53 & 3,75 & 3,32 & 4,05 \\
\cline { 2 - 9 } & 2 & 6,87 & $\mathbf{3 , 1 2}$ & 3,63 & 3,67 & 3,45 & 3,50 & 3,77 \\
\hline \multirow{2}{*}{$50 \%$} & 1 & 7,00 & 3,33 & 3,47 & 3,72 & 3,67 & $\mathbf{3 , 1 5}$ & 3,67 \\
\cline { 2 - 9 } & 2 & 7,00 & 3,80 & $\mathbf{2 , 7 7}$ & 3,57 & 4,82 & 3,50 & 3,45 \\
\hline $70 \%$ & 1 & 5,22 & $\mathbf{3 , 4 7}$ & 3,65 & 3,77 & 4,48 & 3,90 & 2,97 \\
\hline $\begin{array}{c}\text { Frequência } \\
\text { dos menores } \\
\text { "ranks" }\end{array}$ & - & 0 & 3,70 & $\mathbf{3 , 2 7}$ & 3,37 & 3,57 & 3,55 & 3,75 \\
\hline
\end{tabular}

Tabela 4.6: "Ranks" médios em cada cenário de ausência - viés de classificação para a base de dados Íris. Os menores valores de "rank" médios estão destacados em negrito.

para todos os algoritmos avaliados. Observa-se que o algoritmo Média Geral apresentou piores resultados em relação aos demais algoritmos. Para uma melhor visualização, a Figura 4.9(b) apresenta os resultados do viés na classificação, desconsiderando o algoritmo Média Geral. Nesta figura, observa-se que todos os algoritmos apresentam comportamentos semelhantes.

De maneira análoga aos resultados obtidos na avaliação pela predição, os mesmos procedimentos foram adotados para a realização dos testes estatísticos nos resultados obtidos em problemas de classificação. Assim, a Tabela 4.6 apresenta os valores médios dos "ranks" para os resultados da estimação do viés inserido pelos algoritmos de imputação. Neste caso, o algoritmo Média Majority obteve menores valores de "rank" médios na maioria dos cenários de ausência. Os resultados referentes aos totais de vitórias, empates e derrotas nas comparações par-a-par entre os algoritmos são apresentados na Tabela 4.7. No Apêndice se encontra a Tabela A.3, que reporta as comparações par-a-par dos algoritmos de imputação e que foi utilizada para obter os valores totais apresentados na Tabela 4.7. Nesta, pode-se observar que o algoritmo Média Majority obteve melhores resultados para as taxas de $10 \%$ e $30 \%$ de valores ausentes. Nas taxas de $50 \%$ e $70 \%$ de valores ausentes, os algoritmos EACImpute e KNNImpute obtiveram respectivamente os melhores resultados em termos do número de vitórias. Vale observar que o algoritmo que apresentou os melhores resultados na tarefa de predição (IKNNImpute) não se destacou significativamente em termos do viés de classificação, sugerindo que melhores resultados na predição não levam, necessariamente, a um menor viés de classificação.

Diferenças estatísticas entre os algoritmos de imputação foram detectados pelo teste posthoc de Nemenyi, conforme apresentado na Tabela A.4 do Apêndice. Considerando todas as taxas de ausentes, diferenças estatísticas foram encontradas apenas entre o algoritmo Média 


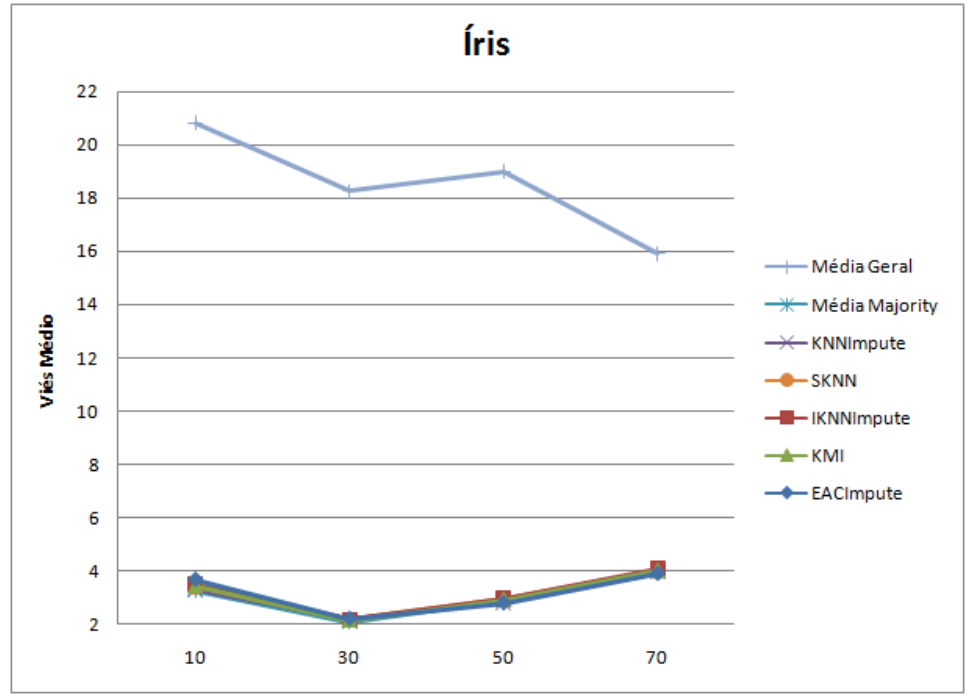

(a)

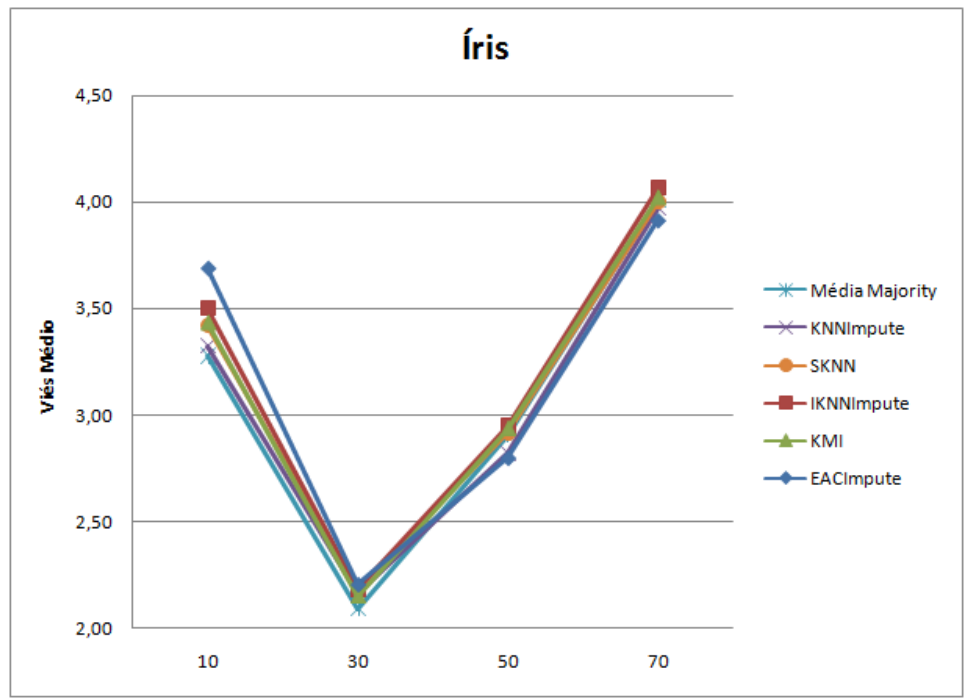

(b)

Figura 4.9: Avaliação por meio da estimação do viés na classificação. Na Figura (a) são apresentados os resultados dos algoritmos comparados com o algoritmo Média Geral. Na Figura (b) são apresentados os resultados dos valores médios do viés, desconsiderando o algoritmo Média Geral.

\begin{tabular}{|c|c|c|c|c|}
\hline Algoritmo & $10 \%$ & $30 \%$ & $50 \%$ & $70 \%$ \\
\hline Média Geral & $(0 / 0 / 12)$ & $(0 / 0 / 12)$ & $(0 / 0 / 12)$ & $(0 / 0 / 12)$ \\
\hline Média Majority & $(12 / 0 / 0)$ & $(11 / 0 / 1)$ & $(9 / 0 / 3)$ & $(8 / 0 / 4)$ \\
\hline KNNImpute & $(10 / 0 / 2)$ & $(7 / 0 / 5)$ & $(9 / 1 / 2)$ & $(10 / 0 / 2)$ \\
\hline SKNN & $(7 / 0 / 5)$ & $(3 / 0 / 9)$ & $(6 / 0 / 6)$ & $(8 / 0 / 4)$ \\
\hline IKNNImpute & $(5 / 0 / 7)$ & $(7 / 1 / 4)$ & $(2 / 0 / 10)$ & $(4 / 0 / 8)$ \\
\hline KMI & $(6 / 0 / 6)$ & $(10 / 0 / 2)$ & $(5 / 1 / 6)$ & $(6 / 0 / 6)$ \\
\hline EACImpute & $(2 / 0 / 10)$ & $(3 / 1 / 8)$ & $(10 / 0 / 2)$ & $(6 / 0 / 6)$ \\
\hline
\end{tabular}

Tabela 4.7: Total de vitórias, empates e derrotas, em relação ao "rank" médio - viés de classificação para a base de dados Íris. 


\begin{tabular}{|c|c|c|c|c|}
\hline Algoritmos & $10 \%$ & $30 \%$ & $50 \%$ & $70 \%$ \\
\hline Média Geral & $(0 / 0 / 12)$ & $(0 / 0 / 12)$ & $(0 / 0 / 12)$ & $(0 / 4 / 8)$ \\
\hline Média Majority & $(2 / 10 / 0)$ & $(2 / 10 / 0)$ & $(2 / 10 / 0)$ & $(2 / 10 / 0)$ \\
\hline KNNImpute & $(2 / 10 / 0)$ & $(2 / 10 / 0)$ & $(2 / 10 / 0)$ & $(1 / 11 / 0)$ \\
\hline SKNN & $(2 / 10 / 0)$ & $(2 / 10 / 0)$ & $(2 / 10 / 0)$ & $(1 / 11 / 0)$ \\
\hline IKNNImpute & $(2 / 10 / 0)$ & $(2 / 10 / 0)$ & $(2 / 10 / 0)$ & $(1 / 11 / 0)$ \\
\hline KMI & $(2 / 10 / 0)$ & $(2 / 10 / 0)$ & $(2 / 10 / 0)$ & $(1 / 11 / 0)$ \\
\hline EACImpute & $(2 / 10 / 0)$ & $(2 / 10 / 0)$ & $(2 / 10 / 0)$ & $(2 / 10 / 0)$ \\
\hline
\end{tabular}

Tabela 4.8: Total de vitórias, empates e derrotas em relação às diferenças estatísticas para cada cenário de ausência - viés de classificação para a base de dados Íris.

Geral (pior algoritmo) e os demais algoritmos. Na Tabela 4.8 são apresentados os resultados da aplicação do teste estatístico considerando-se a soma do número de vitórias, empates e derrotas de cada algoritmo em cada cenário de ausência. Nestes resultados, todos os algoritmos, exceto o algoritmo Média Geral, obtiveram desempenhos semelhantes em todas as taxas de valores ausentes.

Considerando as análises estatísticas realizadas para a capacidade de predição $\left(\overline{E Q_{N}}\right)$, o algoritmo IKNNImpute apresentou melhores resultados em todas as taxas de valores ausentes. Porém, considerando a estimação do viés dos valores imputados em problemas de classificação, todos os algoritmos apresentaram resultados semelhantes, exceto o algoritmo Média Geral. Neste caso, considerando que um aspecto fundamental é analisar a influência dos valores imputados em problemas de classificação, conclui-se que todos os algoritmos, exceto o algoritmo Média Geral, apresentaram desempenhos semelhantes para imputação neste tipo de base de dados. Os resultados obtidos para as demais bases de dados utilizadas neste estudo (Seções 4.5.1.2, 4.5.1.3, 4.5.1.4 e 4.5.1.5) serão analisadas seguindo a mesma metodologia adotada nesta seção.

\subsubsection{Base de Dados "Glass Identification"}

Para a base de dados Glass Identification, os resultados de predição - $\overline{E Q_{N}}$ na Equação 4.1 - nas taxas de 10\%, 30\%, 50\% e 70\% de valores ausentes, são apresentados na Figura 4.10, na qual pode-se observar que a Média Geral (algoritmo mais simples) apresentou melhores resultados nas taxas de $10 \%$ e $30 \%$ de valores ausentes, e todos os algoritmos investigados apresentaram desempenhos semelhantes nos demais casos (50\% e 70\%).

A Tabela 4.9 apresenta os resultados referentes aos valores médios dos "ranks", para cada taxa de valores ausentes e com a quantidade de atributos com valores ausentes, $q_{a}$, variando de 1 até 4 . Por exemplo, pode-se verificar que o EACImpute apresentou melhores resultados (menor "rank") para a taxa de ausentes $10 \%$ e $q_{a}=1$. Os resultados referentes aos totais de vitórias, empates e derrotas de cada algoritmo, na comparação pareada dos valores médios dos "ranks" são apresentados na Tabela 4.10. Nesta tabela, o algoritmo EACImpute apresentou os melhores resultados nas taxas de $10 \%$ e $70 \%$ de valores ausentes e o algoritmo IKNNImpute obteve melhores resultados nas taxas de $30 \%$ e $50 \%$ de valores ausentes. Resultados detalhados referentes ao número de vitórias, empates e derrotas da comparação pareada dos algoritmos 


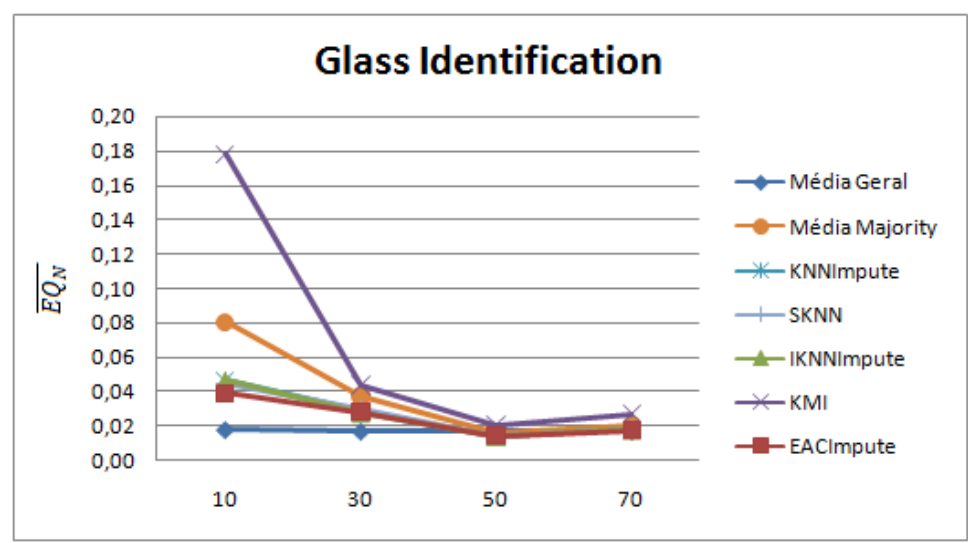

Figura 4.10: Avaliação pelo $\overline{E Q_{N}}$ para a taxa de ausentes em $10 \%, 30 \%, 50 \%$ e $70 \%$.

\begin{tabular}{|c|c|c|c|c|c|c|c|c|}
\hline $\begin{array}{c}\text { Taxa de } \\
\text { Ausentes }\end{array}$ & $\begin{array}{c}\text { Atributos } \\
\text { Ausentes }\left(q_{a}\right)\end{array}$ & Média Geral & Média Majority & KNNImpute & SKNN & IKNNImpute & KMI & EACImpute \\
\hline \multirow{4}{*}{$10 \%$} & 1 & 3,50 & 4,90 & 3,45 & 3,38 & 3,37 & 6,10 & 3,30 \\
\hline & 2 & 1,93 & 5,23 & 4,07 & 3,83 & 3,47 & 6,27 & 3,20 \\
\hline & 3 & 2,37 & 5,77 & 3,57 & 3,60 & 3,17 & 6,27 & 3,27 \\
\hline & 4 & 3,93 & 5,43 & 3,13 & 2,95 & 3,15 & 6,33 & $\mathbf{3 , 0 7}$ \\
\hline \multirow{4}{*}{$30 \%$} & 1 & 1,77 & 5,87 & 3,70 & 3,80 & 3,23 & 6,33 & 3,30 \\
\hline & 2 & 4,10 & 5,20 & $3, \mathbf{0 3}$ & 3,33 & 2,63 & 6,40 & 3,30 \\
\hline & 3 & 4,50 & 5,33 & 2,80 & 3,13 & 3,00 & 6,60 & 2,63 \\
\hline & 4 & 4,93 & 5,33 & 3,33 & 2,37 & 2,30 & 6,43 & 3,30 \\
\hline \multirow{4}{*}{$50 \%$} & 1 & 3,17 & 5,43 & 3,47 & 3,63 & 3,10 & 6,10 & 3,10 \\
\hline & 2 & 4,93 & 5,23 & 2,37 & 3,23 & 2,70 & 6,47 & 3,07 \\
\hline & 3 & 5,13 & 5,33 & 2,47 & 3,27 & 2,57 & 6,70 & 2,53 \\
\hline & 4 & 5,80 & 4,87 & 2,93 & 2,30 & 1,90 & 6,73 & 3,47 \\
\hline \multirow{4}{*}{$70 \%$} & 1 & 3,73 & 5,33 & 3,13 & 3,50 & 2,97 & 6,37 & 2,97 \\
\hline & 2 & 5,47 & 5,07 & 2,73 & 3,07 & 2,87 & 6,87 & 1,93 \\
\hline & 3 & 5,70 & 5,10 & 2,70 & 3,00 & 2,40 & 6,77 & 2,33 \\
\hline & 4 & 6,10 & 4,70 & 3,53 & 2,33 & $\mathbf{1 , 8 0}$ & 6,67 & 2,87 \\
\hline $\begin{array}{c}\text { Frequência } \\
\text { dos menores } \\
\text { "ranks" }\end{array}$ & - & 3 & 0 & 3 & 0 & 5 & 0 & 7 \\
\hline
\end{tabular}

Tabela 4.9: "Ranks" médios em cada cenário de ausência $-\overline{E Q_{N}}$ para a base de dados Glass Identification. Os menores valores de "rank" médios estão destacados em negrito.

podem ser encontrados na Tabela A.5 do Apêndice.

Procedendo-se com os testes de Friedman e de Nemenyi, diferenças estatísticas foram observadas nas comparações pareadas entre alguns algoritmos (Tabela A.6 do Apêndice). Nesta base de dados, existem 24 possíveis combinações (6 comparações pareadas em cada cenário de ausência com 1, 2, 3 e 4 atributos com valores ausentes) em que os algoritmos podem vencer, empatar ou perder. No concernente ao teste de Nemenyi, a Tabela 4.11 apresenta os resultados consolidados (sumarizados tomando-se por base a Tabela A.6 do Apêndice), referentes aos totais de vitórias, empates e derrotas para cada algoritmo. Em resumo, é possível observar que o algoritmo Média Geral apresentou os melhores resultados para a taxa de 10\% de valores ausentes, enquanto que o IKNNImpute se encontra sistematicamente entre os melhores avaliados para os demais cenários.

A Figura 4.11 apresenta os resultados médios sob o ponto de vista da estimação do viés na classificação, obtidos com a aplicação do classificador $k$-NN, o qual obteve maior TCCM para a base de dados Glass Identification, conforme apresentado na Tabela 4.2. Na Figura 


\begin{tabular}{|c|c|c|c|c|}
\hline Algoritmo & $10 \%$ & $30 \%$ & $50 \%$ & $70 \%$ \\
\hline Média Geral & $(16 / 0 / 8)$ & $(12 / 0 / 12)$ & $(9 / 0 / 15)$ & $(5 / 0 / 19)$ \\
\hline Média Majority & $(4 / 0 / 20)$ & $(4 / 0 / 20)$ & $(5 / 0 / 19)$ & $(7 / 0 / 17)$ \\
\hline KNNImpute & $(12 / 0 / 12)$ & $(16 / 0 / 8)$ & $(19 / 0 / 5)$ & $(16 / 0 / 8)$ \\
\hline SKNN & $(15 / 0 / 9)$ & $(13 / 0 / 11)$ & $(13 / 0 / 11)$ & $(14 / 0 / 10)$ \\
\hline IKNNImpute & $(17 / 0 / 7)$ & $(21 / 0 / 3)$ & $(20 / 1 / 3)$ & $(20 / 1 / 3)$ \\
\hline KMI & $(0 / 0 / 24)$ & $(0 / 0 / 24)$ & $(0 / 0 / 24)$ & $(0 / 0 / 24)$ \\
\hline EACImpute & $(20 / 0 / 4)$ & $(18 / 0 / 6)$ & $(17 / 1 / 6)$ & $(21 / 1 / 2)$ \\
\hline
\end{tabular}

Tabela 4.10: Total de vitórias, empates e derrotas em relação ao "rank" médio em cada cenário de ausência $-\overline{E Q_{N}}$ para a base de dados Glass Identification.

\begin{tabular}{|c|c|c|c|c|}
\hline Algoritmo & $10 \%$ & $30 \%$ & $50 \%$ & $70 \%$ \\
\hline Média Geral & $(9 / 15 / 0)$ & $(8 / 9 / 7)$ & $(3 / 9 / 12)$ & $(2 / 9 / 13)$ \\
\hline Média Majority & $(0 / 11 / 13)$ & $(0 / 7 / 17)$ & $(1 / 7 / 16)$ & $(4 / 4 / 16)$ \\
\hline KNNImpute & $(6 / 17 / 1)$ & $(10 / 13 / 1)$ & $(11 / 13 / 0)$ & $(10 / 13 / 1)$ \\
\hline SKNN & $(7 / 16 / 1)$ & $(9 / 14 / 1)$ & $(11 / 13 / 0)$ & $(11 / 13 / 0)$ \\
\hline IKNNImpute & $(7 / 16 / 1)$ & $(10 / 13 / 1)$ & $(12 / 12 / 0)$ & $(12 / 12 / 0)$ \\
\hline KMI & $(0 / 4 / 20)$ & $(0 / 5 / 19)$ & $(0 / 5 / 19)$ & $(0 / 4 / 20)$ \\
\hline EACImpute & $(7 / 17 / 0)$ & $(10 / 13 / 1)$ & $(10 / 13 / 1)$ & $(11 / 13 / 0)$ \\
\hline
\end{tabular}

Tabela 4.11: Total de vitórias, empates e derrotas de cada algoritmo que houve diferença estatística na comparação pareada em cada cenário de ausência $-\overline{E Q_{N}}$ para a base de dados Glass Identification.

4.11(a), novamente, observa-se que o algoritmo Média Geral apresentou piores resultados em relação aos demais algoritmos. Para efeitos de melhor visualização, a Figura 4.11(b) apresenta os resultados do viés na classificação para todos os algoritmos, exceto para o algoritmo Média Geral. Nestes resultados, o algoritmo IKNNImpute apresentou os melhores resultados em todas as taxas de valores ausentes.

A Tabela 4.12 apresenta os valores médios dos "ranks" para os resultados na classificação em cada cenário de ausência. Nestes resultados, pode-se observar que o algoritmo IKNNImpute se destacou na maioria dos cenários de ausência. Os resultados totais das comparações par-a-par dos valores médios dos "ranks" de cada algoritmo são apresentados na Tabela 4.13. Nesta tabela, o algoritmo KNNImpute obteve melhores resultados para a taxa de $10 \%$, enquanto o algoritmo IKNNImpute obteve melhores resultados para demais taxas de valores ausentes. Resultados adicionais (específicos) referentes ao número de vitórias, empates e derrotas dos algoritmos de imputação podem ser encontrados na Tabela A.7 do Apêndice.

Diferenças estatísticas identificadas pelo teste de Nemenyi são apresentadas na Tabela A.8 do Apêndice. Os resultados sumarizados dessa tabela - obtidos pelo somatório do número de vitórias, empates e derrotas - são apresentados na Tabela 4.14. Nesses casos, o algoritmo IKNNImpute obteve os melhores resultados, considerando o número de vitórias, em todos os cenários de valores ausentes.

Nos resultados avaliados pela predição e viés de classificação, o algoritmo IKNNImpute se destacou em relação aos demais algoritmos na maioria dos cenários de ausência. Neste caso, pode-se afirmar que possivelmente existe correlação entre os resultados de predição e de inserção de viés, pois o algoritmo IKNNImpute se destacou em ambas as avaliações. 


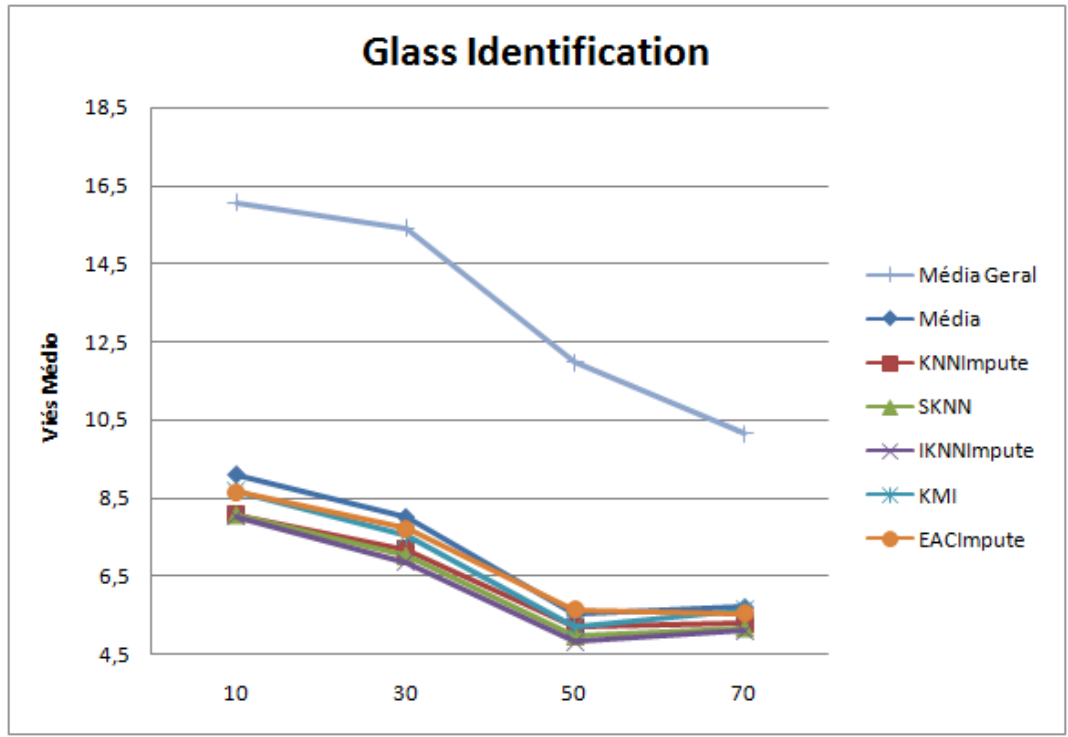

(a)

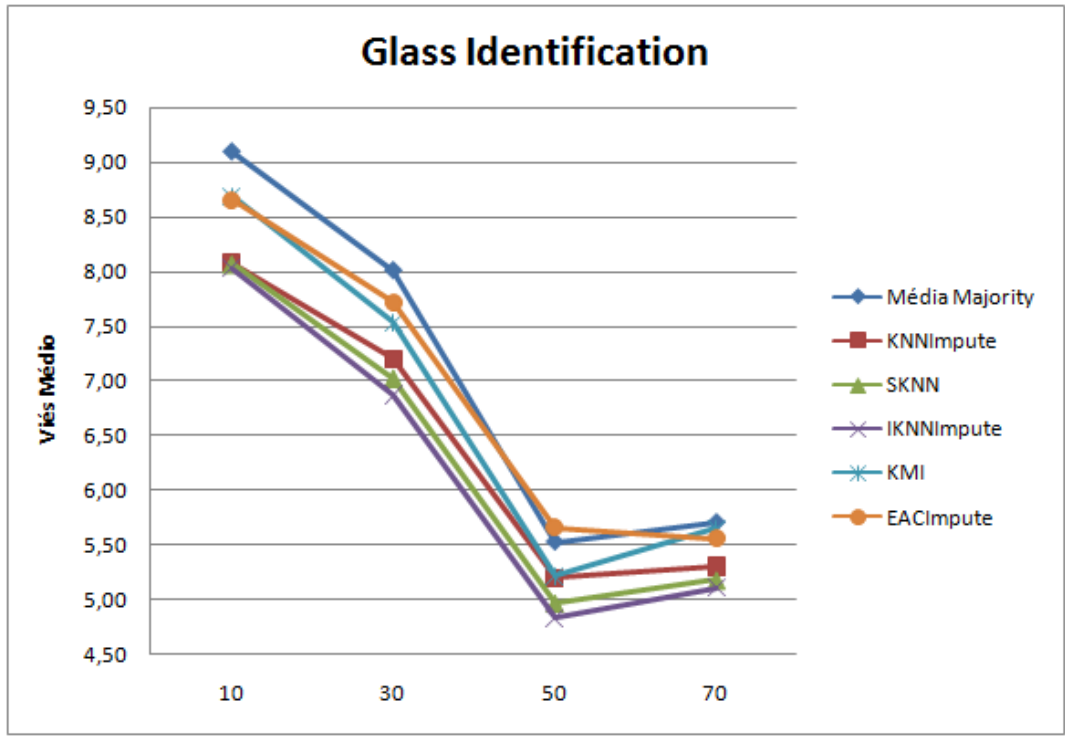

(b)

Figura 4.11: Avaliação pela estimação do viés na classificação para a taxa de ausentes em $10 \%, 30 \%, 50 \%$ e $70 \%$. A Figura (a) apresenta os resultados dos algoritmos comparados com o algoritmo Média geral. A Figura (b) apresenta os resultados do viés na classificação desconsiderando o algoritmo Média geral. 


\begin{tabular}{|c|c|c|c|c|c|c|c|c|}
\hline $\begin{array}{c}\text { Taxa de } \\
\text { Ausentes }\end{array}$ & $\begin{array}{c}\text { Atributos } \\
\text { Ausentes }\left(q_{a}\right)\end{array}$ & Média Geral & Média Majority & KNNImpute & SKNN & IKNNImpute & KMI & EACImpute \\
\hline \multirow{4}{*}{$10 \%$} & 1 & 5,93 & 4,08 & 3,43 & 3,37 & 3,37 & 3,90 & 3,92 \\
\hline & 2 & 5,95 & 4,60 & 2,93 & 3,28 & 3,48 & 4,28 & 3,47 \\
\hline & 3 & 6,78 & 4,92 & 2,77 & 3,08 & 2,92 & 4,08 & 3,45 \\
\hline & 4 & 6,73 & 4,92 & 3,22 & 2,63 & 2,40 & 4,08 & 4,02 \\
\hline \multirow{4}{*}{$30 \%$} & 1 & 6,53 & 4,90 & 3,02 & 3,12 & 2,97 & 4,53 & 2,93 \\
\hline & 2 & 7,00 & 5,08 & 2,87 & 2,63 & 2,30 & 4,15 & 3,97 \\
\hline & 3 & 7,00 & 4,63 & 3,43 & 2,60 & 2,23 & 3,67 & 4,43 \\
\hline & 4 & 7,00 & 5,12 & 3,43 & 2,77 & 1,75 & 3,80 & 4,13 \\
\hline \multirow{4}{*}{$50 \%$} & 1 & 6,22 & 3,85 & 3,73 & 3,23 & 3,20 & 2,97 & 4,80 \\
\hline & 2 & 6,97 & 4,70 & 2,92 & 2,77 & 2,43 & 3,38 & 4,83 \\
\hline & 3 & 6,60 & 5,10 & 3,73 & 2,75 & $\mathbf{1 , 8 3}$ & 3,28 & 4,70 \\
\hline & 4 & 7,00 & 4,63 & 3,88 & 2,97 & 2,33 & 3,58 & 3,60 \\
\hline \multirow{4}{*}{$70 \%$} & 1 & 6,00 & 4,40 & 3,23 & 3,03 & 2,97 & 4,42 & 3,95 \\
\hline & 2 & 5,70 & 4,10 & 3,22 & 2,98 & 3,48 & 4,35 & 4,17 \\
\hline & 3 & 6,83 & 4,57 & 3,17 & 2,95 & 2,48 & 4,23 & 3,77 \\
\hline & 4 & 6,70 & 4,28 & 3,80 & 2,82 & 2,58 & 3,78 & 4,03 \\
\hline $\begin{array}{c}\text { Frequência } \\
\text { dos menores } \\
\text { "ranks" }\end{array}$ & - & 0 & 0 & 3 & 1 & 10 & 1 & 1 \\
\hline
\end{tabular}

Tabela 4.12: "Ranks" médios em cada cenário de ausência - estimação do viés da classificação para a base de dados Glass Identification. Os menores valores de "rank" médios estão destacados em negrito.

\begin{tabular}{|c|c|c|c|c|}
\hline Algoritmo & $10 \%$ & $30 \%$ & $50 \%$ & $70 \%$ \\
\hline Média Geral & $(0 / 0 / 24)$ & $(0 / 0 / 24)$ & $(0 / 0 / 24)$ & $(0 / 0 / 24)$ \\
\hline Média Majority & $(4 / 0 / 20)$ & $(4 / 0 / 20)$ & $(6 / 0 / 18)$ & $(7 / 0 / 17)$ \\
\hline KNNImpute & $(20 / 0 / 4)$ & $(16 / 0 / 8)$ & $(12 / 0 / 12)$ & $(16 / 0 / 8)$ \\
\hline SKNN & $(19 / 1 / 4)$ & $(18 / 0 / 6)$ & $(19 / 0 / 5)$ & $(21 / 0 / 3)$ \\
\hline IKNNImpute & $(19 / 1 / 4)$ & $(23 / 0 / 1)$ & $(23 / 0 / 1)$ & $(22 / 0 / 2)$ \\
\hline KMI & $(9 / 0 / 15)$ & $(10 / 0 / 14)$ & $(17 / 0 / 7)$ & $(8 / 0 / 16)$ \\
\hline EACImpute & $(12 / 0 / 12)$ & $(13 / 0 / 11)$ & $(7 / 0 / 17)$ & $(10 / 0 / 14)$ \\
\hline
\end{tabular}

Tabela 4.13: Total de vitórias, empates e derrotas em relação ao "rank" médio em cada cenário de ausência - estimação do viés de classificação para a base de dados Glass Identification.

\begin{tabular}{|c|c|c|c|c|}
\hline Algoritmos & $10 \%$ & $30 \%$ & $50 \%$ & $70 \%$ \\
\hline Média Geral & $(0 / 1 / 23)$ & $(0 / 0 / 24)$ & $(0 / 1 / 23)$ & $(0 / 2 / 22)$ \\
\hline Média Majority & $(3 / 14 / 7)$ & $(4 / 8 / 12)$ & $(4 / 12 / 8)$ & $(4 / 17 / 3)$ \\
\hline KNNImpute & $(7 / 17 / 0)$ & $(8 / 15 / 1)$ & $(6 / 17 / 1)$ & $(4 / 20 / 0)$ \\
\hline SKNN & $(6 / 18 / 0)$ & $(11 / 13 / 0)$ & $(10 / 14 / 0)$ & $(5 / 19 / 0)$ \\
\hline IKNNImpute & $(8 / 16 / 0)$ & $(15 / 9 / 0)$ & $(11 / 13 / 0)$ & $(7 / 17 / 0)$ \\
\hline KMI & $(4 / 19 / 1)$ & $(4 / 13 / 7)$ & $(7 / 17 / 0)$ & $(3 / 20 / 1)$ \\
\hline EACImpute & $(4 / 19 / 1)$ & $(6 / 14 / 4)$ & $(3 / 12 / 9)$ & $(3 / 21 / 0)$ \\
\hline
\end{tabular}

Tabela 4.14: Total de vitórias, empates e derrotas em relação às diferenças estatísticas na comparação pareada em cada cenário de ausência - estimação do viés na classificação para a base de dados Glass Identification. 


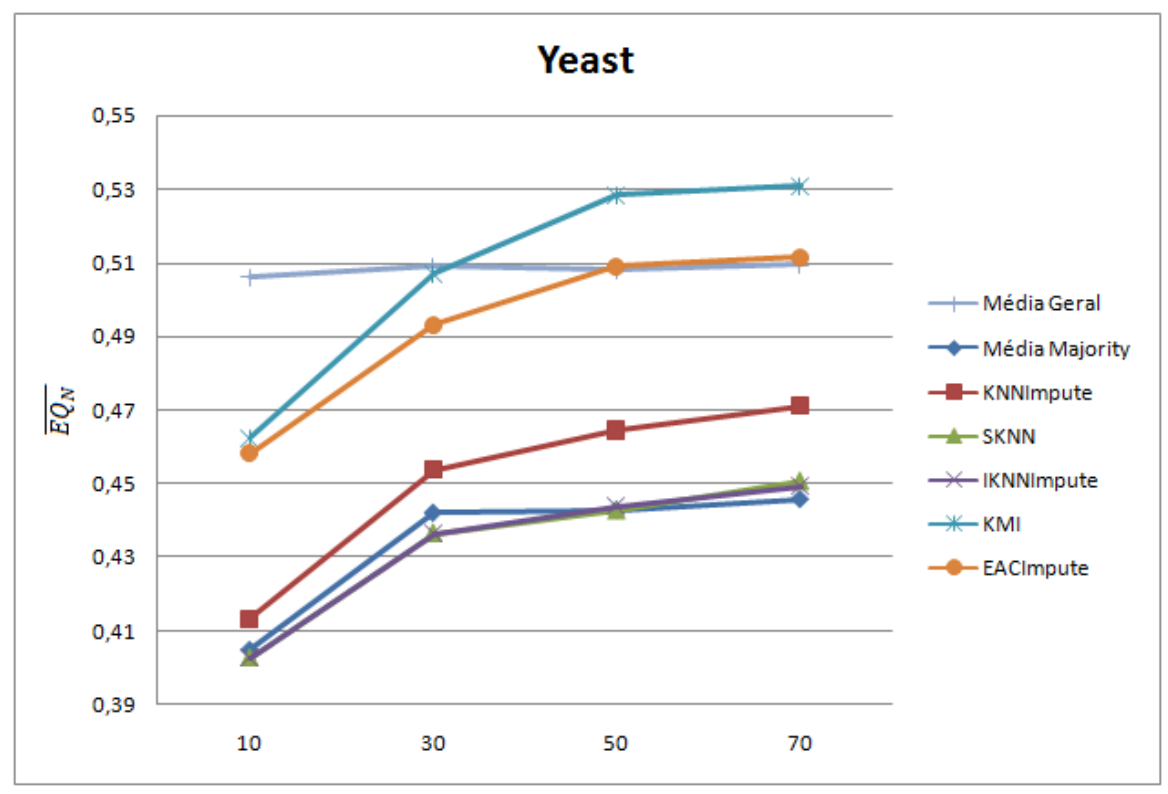

Figura 4.12: Avaliação pelo $\overline{E Q_{N}}$ para a taxa de ausentes em $10 \%, 30 \%, 50 \%$ e $70 \%$.

\subsubsection{Base de Dados "Yeast"}

Para a base de dados Yeast, os resultados referentes aos valores médios da predição com a medida de avaliação $\overline{E Q_{N}}$ em cada taxa de valores ausentes são apresentados na Figura 4.12, na qual pode-se perceber que os algoritmos apresentam comportamentos semelhantes para diferentes taxas de valores ausentes, exceto o algoritmo Média Geral. Pode-se observar também uma pequena distinção entre dois grupos de algoritmos, um baseado em agrupamento (KMI e EACImpute) e o outro baseado no $k$-NN (KNNImpute, SKNN e IKNNImpute). O grupo contendo os algoritmos baseados no $k$-NN e o Média Majority apresentou melhores resultados em todas as taxas de valores ausentes. A Tabela 4.15 apresenta os valores médios dos "ranks" obtidos com base na avaliação dos valores do $\overline{E Q_{N}}$. Esta tabela sugere um equilíbrio de desempenho entre os algoritmos Média Majority, KNNImpute, SKNN e IKNNImpute.

Da mesma forma que na base de dados Glass Identification, existem 24 possíveis combinações em que os algoritmos podem vencer, empatar ou perder - tais resultados são apresentados na Tabela A.9 do Apêndice. Com base nesta tabela, a Tabela 4.16 apresenta os resultados referentes aos totais de vitórias, empates e derrotas para cada algoritmo. Nestas comparações, os algoritmos SKNN e o IKNNImpute apresentaram os melhores resultados nas taxas de $10 \%$ e $30 \%$ de valores ausentes, enquanto que o algoritmo SKNN obteve melhores resultados na taxa de $50 \%$ de valores ausentes. Finalmente, para a taxa de $70 \%$ de valores ausentes o algoritmo Média Majority obteve melhores resultados.

Procedendo-se com os testes de Friedman e de Nemenyi, foram observadas diferenças estatísticas nas comparações pareadas entre os algoritmos, conforme apresentado na Tabela A.10 do Apêndice. A Tabela 4.17 apresenta os resultados consolidados, referentes aos totais de vitórias, empates e derrotas dos resultados reportados na Tabela A.10. Estes resultados suge- 


\begin{tabular}{|c|c|c|c|c|c|c|c|c|}
\hline $\begin{array}{c}\text { Taxa de } \\
\text { Ausentes }\end{array}$ & $\begin{array}{c}\text { Atributos } \\
\text { Ausentes }\left(q_{a}\right)\end{array}$ & Média Geral & Média Majority & KNNImpute & SKNN & IKNNImpute & KMI & EACImpute \\
\hline \multirow{4}{*}{$10 \%$} & 1 & 4,67 & 4,00 & 3,30 & 3,17 & 2,67 & 5,40 & 4,80 \\
\hline & 2 & 5,60 & 3,23 & 3,00 & 2,70 & 2,77 & 5,30 & 5,40 \\
\hline & 3 & 5,97 & 3,23 & 4,23 & 2,40 & 2,33 & 4,87 & 4,97 \\
\hline & 4 & 6,03 & 2,20 & 4,60 & 2,47 & 3,13 & 4,80 & 4,77 \\
\hline \multirow{4}{*}{$30 \%$} & 1 & 5,73 & 3,30 & 2,63 & 2,83 & 2,57 & 5,73 & 5,20 \\
\hline & 2 & 6,00 & 3,50 & 2,30 & 2,70 & 2,57 & 5,77 & 5,17 \\
\hline & 3 & 6,17 & 2,50 & 4,30 & 1,83 & 2,03 & 6,20 & 4,97 \\
\hline & 4 & 6,17 & 2,20 & 5,00 & 2,13 & 2,43 & 5,07 & 5,00 \\
\hline \multirow{4}{*}{$50 \%$} & 1 & 5,53 & 2,87 & 2,33 & 2,77 & 2,83 & 6,37 & 5,30 \\
\hline & 2 & 5,87 & 2,77 & 3,03 & 2,03 & 2,37 & 6,27 & 5,67 \\
\hline & 3 & 5,60 & 2,07 & 4,37 & 1,90 & 2,07 & 6,27 & 5,73 \\
\hline & 4 & 5,40 & 1,50 & 4,53 & 2,00 & 2,50 & 5,87 & 6,20 \\
\hline \multirow{4}{*}{$70 \%$} & 1 & 6,03 & 2,73 & 2,67 & 2,70 & 2,83 & 5,57 & 5,47 \\
\hline & 2 & 6,07 & 2,23 & 3,50 & 2,57 & 1,97 & 6,10 & 5,57 \\
\hline & 3 & 5,67 & 1,73 & 4,33 & 2,03 & 2,40 & 6,27 & 5,57 \\
\hline & 4 & 5,33 & 1,43 & 4,57 & 2,27 & 2,37 & 6,07 & 5,97 \\
\hline $\begin{array}{l}\text { Frequência } \\
\text { dos menores } \\
\text { "ranks" }\end{array}$ & - & 0 & 4 & 3 & 5 & 4 & 0 & 0 \\
\hline
\end{tabular}

Tabela 4.15: "Ranks" médios em cada cenário de ausência $-\overline{E Q_{N}}$ para a base de dados Yeast. Os menores valores de "rank" médios estão destacados em negrito.

\begin{tabular}{|c|c|c|c|c|}
\hline Algoritmo & $10 \%$ & $30 \%$ & $50 \%$ & $70 \%$ \\
\hline Média Geral & $(2 / 0 / 22)$ & $(1 / 1 / 22)$ & $(6 / 0 / 18)$ & $(4 / 0 / 20)$ \\
\hline Média Majority & $(16 / 0 / 8)$ & $(15 / 0 / 9)$ & $(17 / 1 / 6)$ & $(21 / 0 / 3)$ \\
\hline KNNImpute & $(14 / 0 / 10)$ & $(16 / 1 / 7)$ & $(15 / 0 / 9)$ & $(15 / 0 / 9)$ \\
\hline SKNN & $(21 / 0 / 3)$ & $(20 / 0 / 4)$ & $(22 / 0 / 2)$ & $(19 / 0 / 5)$ \\
\hline IKNNImpute & $(21 / 0 / 3)$ & $(20 / 0 / 4)$ & $(17 / 1 / 6)$ & $(17 / 0 / 7)$ \\
\hline KMI & $(5 / 0 / 19)$ & $(2 / 1 / 21)$ & $(1 / 0 / 23)$ & $(1 / 0 / 23)$ \\
\hline EACImpute & $(5 / 0 / 19)$ & $(8 / 1 / 15)$ & $(5 / 0 / 19)$ & $(7 / 0 / 17)$ \\
\hline
\end{tabular}

Tabela 4.16: Total de vitórias, empates e derrotas, em relação aos "ranks" médios relativos ao $\overline{E Q_{N}}$ para a base de dados Yeast. 


\begin{tabular}{|c|c|c|c|c|}
\hline Algoritmo & $10 \%$ & $30 \%$ & $50 \%$ & $70 \%$ \\
\hline Média Geral & $(0 / 12 / 12)$ & $(0 / 9 / 15)$ & $(0 / 10 / 14)$ & $(0 / 10 / 14)$ \\
\hline Média Majority & $(10 / 14 / 0)$ & $(14 / 10 / 0)$ & $(14 / 10 / 0)$ & $(14 / 10 / 0)$ \\
\hline KNNImpute & $(5 / 14 / 5)$ & $(8 / 10 / 6)$ & $(8 / 10 / 6)$ & $(7 / 11 / 6)$ \\
\hline SKNN & $(13 / 11 / 0)$ & $(14 / 10 / 0)$ & $(14 / 10 / 0)$ & $(14 / 10 / 0)$ \\
\hline IKNNImpute & $(14 / 10 / 0)$ & $(14 / 10 / 0)$ & $(14 / 10 / 0)$ & $(14 / 10 / 0)$ \\
\hline KMI & $(0 / 11 / 13)$ & $(0 / 9 / 15)$ & $(0 / 9 / 15)$ & $(0 / 9 / 15)$ \\
\hline EACImpute & $(0 / 12 / 12)$ & $(0 / 10 / 14)$ & $(0 / 9 / 15)$ & $(0 / 10 / 14)$ \\
\hline
\end{tabular}

Tabela 4.17: Total de vitórias, empates e derrotas de cada algoritmo que houve diferença estatística na comparação pareada em cada cenário de ausência $-\overline{E Q_{N}}$ para a base de dados Yeast.

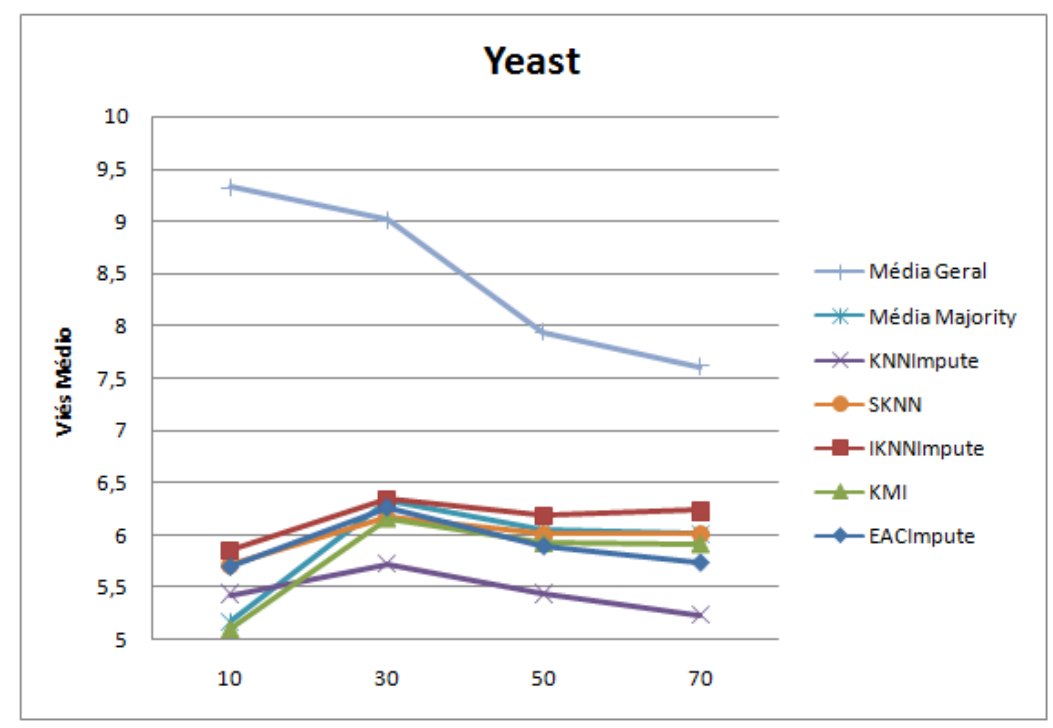

Figura 4.13: Resultados médios da avaliação pela estimação do viés na classificação para a taxa de ausentes em 10\%, 30\%, 50\% e $70 \%$.

rem que os algoritmos IKNNImpute, SKNN e Média Majority se destacaram em relação aos demais e, concomitantemente, apresentam grande equilíbrio entre si.

A Figura 4.13 apresenta os resultados do viés das imputações na tarefa de classificação. Nestes resultados todos os algoritmos, exceto o algoritmo Média Geral, apresentam um comportamento parecido. Estes resultados foram obtidos utilizando o algoritmo de classificação MultiLayer Perceptron (MLP) que obteve uma taxa de classificação correta média superior aos demais algoritmos para esta base (Tabela 4.2). A Tabela 4.18, por sua vez, apresenta os valores médios dos "ranks" obtidos em relação ao viés inserido em problemas de classificação. Nesta tabela, pode-se observar que os algoritmos KNNImpute e EACImpute apresentam os melhores resultados. Resultados referentes ao numero de vitórias, empates e derrotas dos algoritmos em cada cenário de ausência são apresentados na Tabela A.11 do Apêndice. A Tabela 4.19 sumariza os resultados da Tabela A.11 do Apêndice, apresentando os resultados obtidos por meio do somatório do número de vitórias, empates e derrotas das comparações realizadas. Nesta tabela, o algoritmo Média Majority e o KMI obtiveram melhores resultados para a taxa de $10 \%$ de valores ausentes, seguido do algoritmo KNNImpute nas demais taxas de valores ausentes.

A Tabela A.12 do Apêndice reporta os resultados detalhados referentes às diferenças es- 


\begin{tabular}{|c|c|c|c|c|c|c|c|c|}
\hline $\begin{array}{c}\text { Taxa de } \\
\text { Ausentes }\end{array}$ & $\begin{array}{c}\text { Atributos } \\
\text { Ausentes }\left(q_{a}\right)\end{array}$ & Média Geral & Média Majority & KNNImpute & SKNN & IKNNImpute & KMI & EACImpute \\
\hline \multirow{4}{*}{$10 \%$} & 1 & 4,20 & 3,57 & 4,08 & 4,45 & 4,57 & 3,38 & 3,75 \\
\hline & 2 & 5,03 & 3,28 & 3,65 & 4,47 & 4,60 & 3,63 & 3,33 \\
\hline & 3 & 6,03 & 2,83 & 3,67 & 4,13 & 4,47 & 3,02 & 3,85 \\
\hline & 4 & 5,77 & 3,18 & 2,97 & 4,33 & 4,75 & 2,37 & 4,63 \\
\hline \multirow{4}{*}{$30 \%$} & 1 & 4,10 & 3,98 & 4,12 & 4,38 & 4,47 & 3,93 & 3,02 \\
\hline & 2 & 5,50 & 3,82 & 3,25 & 4,08 & 4,43 & 3,70 & 3,22 \\
\hline & 3 & 5,67 & 4,10 & 2,05 & 3,77 & 4,78 & 3,57 & 4,07 \\
\hline & 4 & 6,10 & 4,72 & 2,08 & 3,03 & 4,00 & 3,68 & 4,38 \\
\hline \multirow{4}{*}{$50 \%$} & 1 & 4,40 & 4,05 & 3,30 & 4,22 & 4,82 & 4,22 & 3,00 \\
\hline & 2 & 4,47 & 3,62 & 3,35 & 4,62 & 5,17 & 3,40 & 3,38 \\
\hline & 3 & 5,77 & 4,35 & 1,90 & 3,58 & 4,82 & 3,80 & 3,78 \\
\hline & 4 & 5,93 & 4,40 & 2,10 & 3,35 & 4,38 & 3,63 & 4,20 \\
\hline \multirow{4}{*}{$70 \%$} & 1 & 4,20 & 3,60 & 3,83 & 4,40 & 4,80 & 4,07 & 3,10 \\
\hline & 2 & 4,43 & 3,90 & 2,58 & 4,67 & 5,37 & 3,90 & 3,15 \\
\hline & 3 & 5,03 & 4,37 & 1,62 & 3,87 & 5,17 & 3,97 & 3,98 \\
\hline & 4 & 6,27 & 4,30 & $\mathbf{1 , 5 5}$ & 3,50 & 4,77 & 3,43 & 4,18 \\
\hline $\begin{array}{c}\text { Frequência } \\
\text { dos menores } \\
\text { "ranks" }\end{array}$ & - & 0 & 2 & 8 & 0 & 0 & 2 & 4 \\
\hline
\end{tabular}

Tabela 4.18: "Ranks" médios em cada cenário de ausência - estimação do viés da classificação para a base de dados Yeast. Os menores valores de "rank" médios estão destacados em negrito.

\begin{tabular}{|c|c|c|c|c|}
\hline Algoritmo & $10 \%$ & $30 \%$ & $50 \%$ & $70 \%$ \\
\hline Média Geral & $(2 / 0 / 22)$ & $(3 / 0 / 21)$ & $(3 / 0 / 21)$ & $(5 / 0 / 19)$ \\
\hline Média Majority & $(21 / 0 / 3)$ & $(10 / 0 / 14)$ & $(10 / 0 / 14)$ & $(12 / 1 / 11)$ \\
\hline KNNImpute & $(15 / 0 / 9)$ & $(19 / 0 / 5)$ & $(23 / 0 / 1)$ & $(22 / 0 / 2)$ \\
\hline SKNN & $(8 / 0 / 16)$ & $(12 / 0 / 12)$ & $(13 / 1 / 10)$ & $(11 / 0 / 13)$ \\
\hline IKNNImpute & $(3 / 0 / 21)$ & $(5 / 0 / 19)$ & $(3 / 0 / 21)$ & $(1 / 0 / 23)$ \\
\hline KMI & $(21 / 0 / 3)$ & $(18 / 0 / 6)$ & $(13 / 1 / 10)$ & $(15 / 1 / 8)$ \\
\hline EACImpute & $(14 / 0 / 10)$ & $(17 / 0 / 7)$ & $(18 / 0 / 6)$ & $(17 / 0 / 7)$ \\
\hline
\end{tabular}

Tabela 4.19: Total de vitórias, empates e derrotas, em relação aos "ranks" médios relativos à estimação do viés na classificação para a base de dados Yeast. 


\begin{tabular}{|c|c|c|c|c|}
\hline Algoritmos & $10 \%$ & $30 \%$ & $50 \%$ & $70 \%$ \\
\hline Média Geral & $(0 / 14 / 10)$ & $(0 / 10 / 14)$ & $(0 / 14 / 10)$ & $(0 / 17 / 7)$ \\
\hline Média Majority & $(5 / 19 / 0)$ & $(2 / 19 / 3)$ & $(1 / 21 / 2)$ & $(1 / 21 / 2)$ \\
\hline KNNImpute & $(4 / 20 / 0)$ & $(12 / 12 / 0)$ & $(12 / 22 / 0)$ & $(15 / 9 / 0)$ \\
\hline SKNN & $(1 / 22 / 1)$ & $(3 / 20 / 1)$ & $(2 / 21 / 1)$ & $(1 / 20 / 3)$ \\
\hline IKNNImpute & $(0 / 21 / 3)$ & $(1 / 21 / 2)$ & $(1 / 17 / 6)$ & $(0 / 19 / 5)$ \\
\hline KMI & $(5 / 19 / 0)$ & $(3 / 19 / 2)$ & $(3 / 19 / 2)$ & $(1 / 21 / 2)$ \\
\hline EACImpute & $(2 / 19 / 3)$ & $(3 / 19 / 2)$ & $(4 / 18 / 2)$ & $(3 / 19 / 2)$ \\
\hline
\end{tabular}

Tabela 4.20: Total de vitórias, empates e derrotas de cada algoritmo que houve diferença estatística na comparação pareada em cada cenário de ausência - estimação do viés na classificação para a base de dados Yeast.

tatísticas identificadas pelo teste de Nemenyi, em cada cenário de ausência, realizadas pelas comparações pareadas dos algoritmos, enquanto que a Tabela 4.20 sumariza tais resultados em termos do número total de vitórias, empates e derrotas. Nesses casos, Média Majority e KMI apresentaram os melhores resultados na taxa de $10 \%$ de valores ausentes, enquanto que nas demais taxas de valores ausentes o algoritmo KNNImpute obteve melhores resultados.

Em resumo, considerando os resultados obtidos para o $\overline{E Q_{N}}$ com o teste estatístico de Nemenyi, observa-se que os algoritmos IKNNImpute, SKNN e Média Majority apresentaram melhor desempenho. Considerando a tarefa de classificação, o algoritmo KNNImpute obteve melhores resultados. Neste caso, pode-se afirmar que possivelmente existe correlação entre os resultados de predição e de inserção de viés, pois o algoritmo KNNImpute se destacou em ambas as avaliações.

\subsubsection{Base de dados "Pen-Digits"}

A Figura 4.14 ilustra os resultados referentes aos valores médios para a medida $\overline{E Q_{N}}$ nas taxas de $10 \%, 30 \%, 50 \%$ e $70 \%$ de valores ausentes. Nestes resultados, todos os algoritmos apresentam comportamentos semelhantes em todas as taxas de valores ausentes, com um destaque para os algoritmos KNNImpute, SKNN e IKNNImpute, os quais apresentam menores valores para o erro de predição.

A Tabela 4.21 reporta os valores médios dos "ranks" para a quantidade de atributos com valores ausentes, $q_{a}$, variando entre 1 e 8 . O algoritmo IKNNImpute apresentou os melhores resultados nesta avaliação seguido dos algoritmos KNNImpute e SKNN. A Tabela 4.22 apresenta os resultados referentes ao números totais de vitórias, empates e derrotas de cada algoritmo comparado por meio dos valores médios dos "ranks". Nesta tabela, os algoritmos KNNImpute, SKNN e o IKNNImpute apresentaram os melhores resultados na taxa de $10 \%$ de valores ausentes, enquanto o algoritmo IKNNImpute obteve melhores resultados nas demais taxas de valores ausentes. Resultados detalhados relativos aos valores do número de vitórias, empates e derrotas dos algoritmos de imputação são encontrados na Tabela A.13 do Apêndice.

Nesta base de dados existem 48 possíveis combinações ( 6 comparações pareadas em cada cenário de ausência com $\left.q_{a}=1, \ldots, 8\right)$ nos quais os algoritmos podem vencer, empatar ou perder. As diferenças estatísticas em relação aos desempenhos dos algoritmos identificadas por 


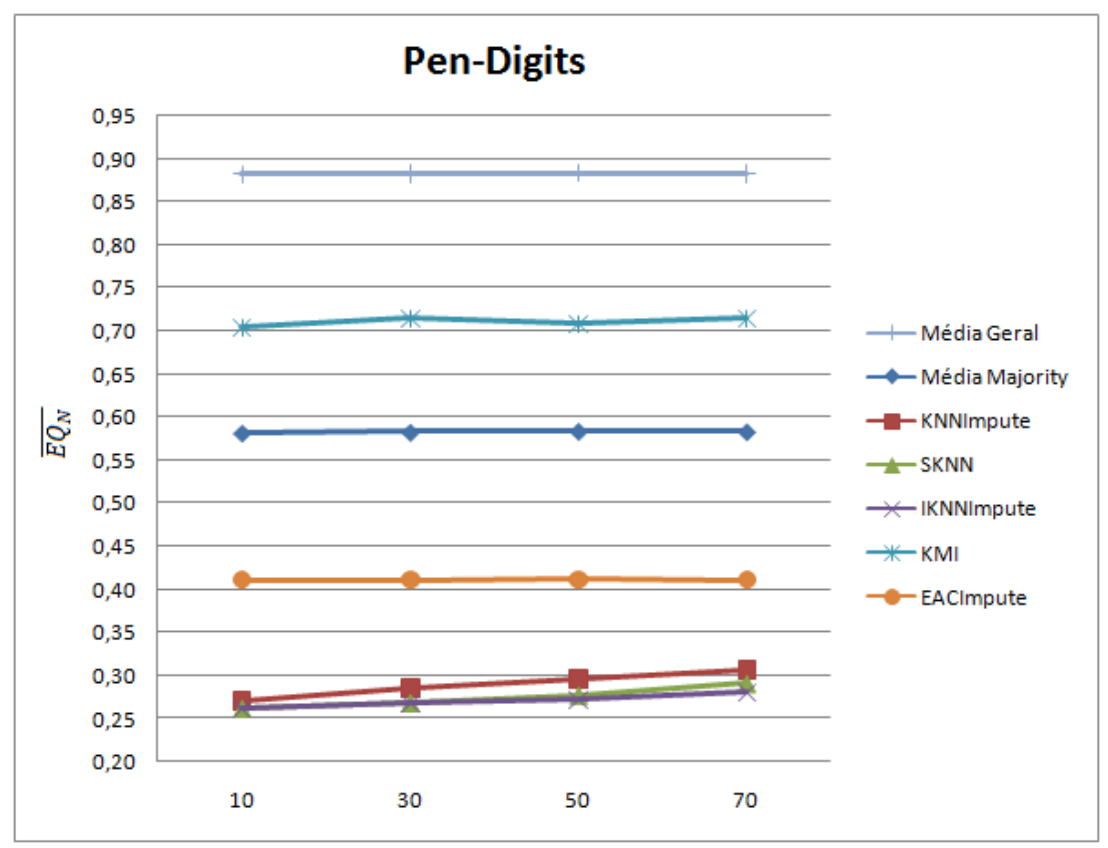

Figura 4.14: Avaliação pelo $\overline{E Q_{N}}$ para a taxa de ausentes em $10 \%, 30 \%, 50 \%$ e $70 \%$.

\begin{tabular}{|c|c|c|c|c|c|c|c|c|}
\hline $\begin{array}{c}\text { Taxa de } \\
\text { Ausentes }\end{array}$ & $\begin{array}{c}\text { Atributos } \\
\text { Ausentes }\left(q_{a}\right)\end{array}$ & Média Geral & Média Majority & KNNImpute & SKNN & IKNNImpute & KMI & EACImpute \\
\hline \multirow{8}{*}{$10 \%$} & 1 & 7,00 & 5,00 & 1,83 & 2,43 & 1,73 & 6,00 & 4,00 \\
\hline & 2 & 7,00 & 5,00 & 1,53 & 2,63 & 1,83 & 6,00 & 4,00 \\
\hline & 3 & 7,00 & 5,00 & 1,60 & 2,63 & 1,77 & 6,00 & 4,00 \\
\hline & 4 & 7,00 & 5,00 & 1,67 & 2,50 & 1,83 & 6,00 & 4,00 \\
\hline & 5 & 7,00 & 5,00 & 2,07 & 1,83 & 2,10 & 6,00 & 4,00 \\
\hline & 6 & 7,00 & 5,00 & 2,97 & 1,43 & 1,60 & 6,00 & 4,00 \\
\hline & 7 & 7,00 & 5,00 & 3,00 & 1,37 & 1,63 & 6,00 & 4,00 \\
\hline & 8 & 7,00 & 5,00 & 3,00 & 1,40 & 1,60 & 6,00 & 4,00 \\
\hline \multirow{8}{*}{$30 \%$} & 1 & 7,00 & 5,00 & 1,93 & 2,97 & 1,10 & 6,00 & 4,00 \\
\hline & 2 & 7,00 & 5,00 & 1,57 & 2,97 & 1,47 & 6,00 & 4,00 \\
\hline & 3 & 7,00 & 5,00 & 1,27 & 3,00 & 1,73 & 6,00 & 4,00 \\
\hline & 4 & 7,00 & 5,00 & 1,13 & 2,80 & 2,07 & 6,00 & 4,00 \\
\hline & 5 & 7,00 & 5,00 & 2,43 & 1,87 & 1,70 & 6,00 & 4,00 \\
\hline & 6 & 7,00 & 5,00 & 3,00 & 1,63 & 1,37 & 6,00 & 4,00 \\
\hline & 7 & 7,00 & 5,00 & 3,00 & 1,30 & 1,70 & 6,00 & 4,00 \\
\hline & 8 & 7,00 & 5,00 & 3,00 & 1,17 & 1,83 & 6,00 & 4,00 \\
\hline \multirow{8}{*}{$50 \%$} & 1 & 7,00 & 5,00 & 1,97 & 3,00 & 1,03 & 6,00 & 4,00 \\
\hline & 2 & 7,00 & 5,00 & 1,70 & 3,00 & 1,30 & 6,00 & 4,00 \\
\hline & 3 & 7,00 & 5,00 & 1,20 & 2,97 & 1,83 & 6,00 & 4,00 \\
\hline & 4 & 7,00 & 5,00 & 1,10 & 2,90 & 2,00 & 6,00 & 4,00 \\
\hline & 5 & 7,00 & 5,00 & 1,73 & 2,77 & 1,50 & 6,00 & 4,00 \\
\hline & 6 & 7,00 & 5,00 & 3,00 & 1,77 & 1,23 & 6,00 & 4,00 \\
\hline & 7 & 7,00 & 5,00 & 3,00 & 1,70 & 1,30 & 6,00 & 4,00 \\
\hline & 8 & 7,00 & 5,00 & 3,03 & 1,53 & 1,47 & 6,00 & 3,97 \\
\hline \multirow{8}{*}{$70 \%$} & 1 & 7,00 & 5,00 & 2,00 & 3,00 & 1,00 & 6,00 & 4,00 \\
\hline & 2 & 7,00 & 5,00 & 1,83 & 3,00 & 1,17 & 6,00 & 4,00 \\
\hline & 3 & 7,00 & 5,00 & 1,23 & 3,00 & 1,77 & 6,00 & 4,00 \\
\hline & 4 & 7,00 & 5,00 & $\mathbf{1 , 0 7}$ & 3,00 & 1,93 & 6,00 & 4,00 \\
\hline & 5 & 7,00 & 5,00 & 1,40 & 3,00 & 1,60 & 6,00 & 4,00 \\
\hline & 6 & 7,00 & 5,00 & 3,00 & 2,00 & 1,00 & 6,00 & 4,00 \\
\hline & 7 & 7,00 & 5,00 & 3,00 & 1,93 & 1,07 & 6,00 & 4,00 \\
\hline & 8 & 7,00 & 5,00 & 3,60 & 1,77 & 1,23 & 6,00 & 3,40 \\
\hline $\begin{array}{c}\text { Frequência } \\
\text { dos menores } \\
\text { "ranks" }\end{array}$ & - & 0 & 0 & 10 & 6 & 16 & 0 & 0 \\
\hline
\end{tabular}

Tabela 4.21: "Ranks" médios em cada cenário de ausência $-\overline{E Q_{N}}$ para a base de dados PenDigits. Os menores valores de "rank" médios estão destacados em negrito. 


\begin{tabular}{|c|c|c|c|c|}
\hline Algoritmo & $10 \%$ & $30 \%$ & $50 \%$ & $70 \%$ \\
\hline Média Geral & $(0 / 0 / 48)$ & $(0 / 0 / 48)$ & $(0 / 0 / 48)$ & $(0 / 0 / 48)$ \\
\hline Média Majority & $(16 / 0 / 32)$ & $(16 / 0 / 32)$ & $(16 / 0 / 32)$ & $(16 / 0 / 32)$ \\
\hline KNNImpute & $(40 / 0 / 8)$ & $(38 / 0 / 10)$ & $(39 / 0 / 9)$ & $(39 / 0 / 9)$ \\
\hline SKNN & $(40 / 0 / 8)$ & $(38 / 0 / 10)$ & $(35 / 0 / 13)$ & $(35 / 0 / 13)$ \\
\hline IKNNImpute & $(40 / 0 / 8)$ & $(44 / 0 / 4)$ & $(46 / 0 / 2)$ & $(45 / 0 / 3)$ \\
\hline KMI & $(8 / 0 / 40)$ & $(8 / 0 / 40)$ & $(8 / 0 / 40)$ & $(8 / 0 / 40)$ \\
\hline EACImpute & $(24 / 0 / 24)$ & $(24 / 0 / 24)$ & $(24 / 0 / 24)$ & $(25 / 0 / 23)$ \\
\hline
\end{tabular}

Tabela 4.22: Total de vitórias, empates e derrotas, em relação aos "ranks" médios relativos ao $\overline{E Q_{N}}$ para a base de dados Pen-Digits.

\begin{tabular}{|c|c|c|c|c|}
\hline Algoritmo & $10 \%$ & $30 \%$ & $50 \%$ & $70 \%$ \\
\hline Média Geral & $(0 / 8 / 40)$ & $(0 / 8 / 40)$ & $(0 / 8 / 40)$ & $(0 / 8 / 40)$ \\
\hline Média Majority & $(8 / 16 / 24)$ & $(8 / 16 / 24)$ & $(8 / 16 / 24)$ & $(8 / 15 / 25)$ \\
\hline KNNImpute & $(29 / 13 / 6)$ & $(32 / 12 / 4)$ & $(31 / 13 / 4)$ & $(32 / 12 / 4)$ \\
\hline SKNN & $(35 / 13 / 0)$ & $(31 / 12 / 5)$ & $(28 / 16 / 4)$ & $(28 / 14 / 6)$ \\
\hline IKNNImpute & $(35 / 13 / 0)$ & $(35 / 13 / 0)$ & $(37 / 11 / 0)$ & $(38 / 10 / 0)$ \\
\hline KMI & $(0 / 16 / 32)$ & $(0 / 16 / 32)$ & $(0 / 16 / 32)$ & $(0 / 16 / 32)$ \\
\hline EACImpute & $(16 / 11 / 21)$ & $(16 / 15 / 17)$ & $(16 / 16 / 16)$ & $(17 / 15 / 16)$ \\
\hline
\end{tabular}

Tabela 4.23: Total de vitórias, empates e derrotas de cada algoritmo que houve diferença estatística na comparação pareada em cada cenário de ausência $-\overline{E Q_{N}}$ para a base de dados Pen-Digits.

meio do teste de Nemenyi para cada cenário são apresentadas na Tabela A.14 do Apêndice. A Tabela 4.23 apresenta os resultados referentes ao somatório do número de vitórias, empates e derrotas de cada algoritmo apresentado na Tabela A.14. Na Tabela 4.23, observa-se que para a taxa de $10 \%$ de ausentes os algoritmos SKNN e IKNNImpute apresentaram melhores resultados, obtendo o maior número de vitórias. Nas demais taxas de valores ausentes o algoritmo IKNNImpute obteve melhores resultados.

A Figura 4.15 apresenta os resultados médios sob o ponto de vista do viés na classificação. Estes resultados foram obtidos por meio do algoritmo J4.8 (baseado em árvore de decisão) que apresentou melhor resultado de taxa de classificação correta média para esta base, conforme reportado na Tabela 4.2. Observa-se que na Figura 4.15(a) o algoritmo Média Geral apresentou os piores resultados. Para uma melhor visualização dos resultados, a Figura 4.15(b) apresenta os resultados para todos os algoritmos, exceto para o algoritmo Média Geral. Nestes resultados os algoritmos KNNImpute, SKNN, IKNNImpute obtiveram os melhores resultados.

A Tabela 4.24 reporta os valores médios dos "ranks" obtidos por meio da estimação do viés na classificação. Nestes resultados, os melhores resultados foram alcançados pelos algoritmos KNNImpute, SKNN e EACImpute respectivamente. A Tabela 4.25 sumariza tais resultados, apresentando os somatórios do número de vitórias, empates e derrotas de cada algoritmo analisado. Nesta tabela, o algoritmo SKNN obteve melhores resultados para a taxa de $10 \%$ de valores ausentes e o algoritmo KNNImpute fornece melhores resultados para as demais taxas de valores ausentes.

Os cenários de ausência para os quais foram observadas diferenças estatísticas são reportados na Tabela A.16 do Apêndice. A Tabela 4.26 sumariza tais resultados por meio do somatório do número de vitórias, empates e derrotas. Nesses casos, os algoritmos KNNImpute e SKNN 


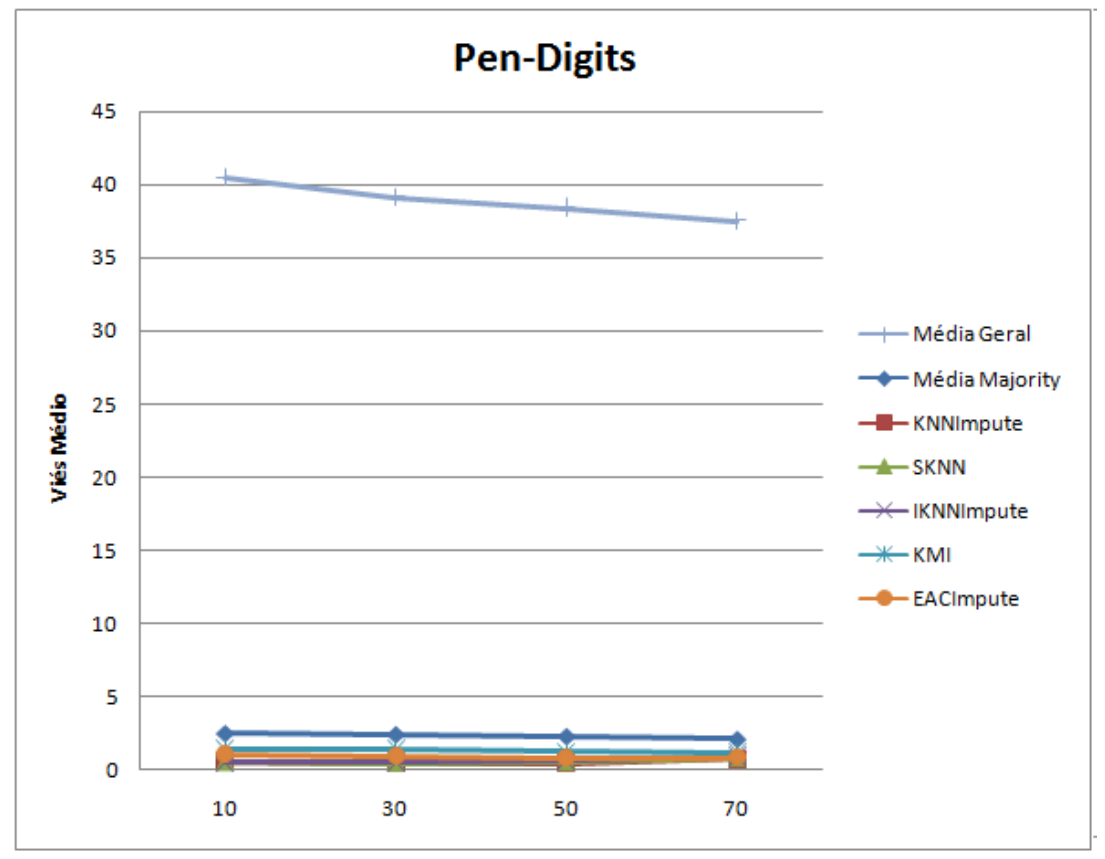

(a)

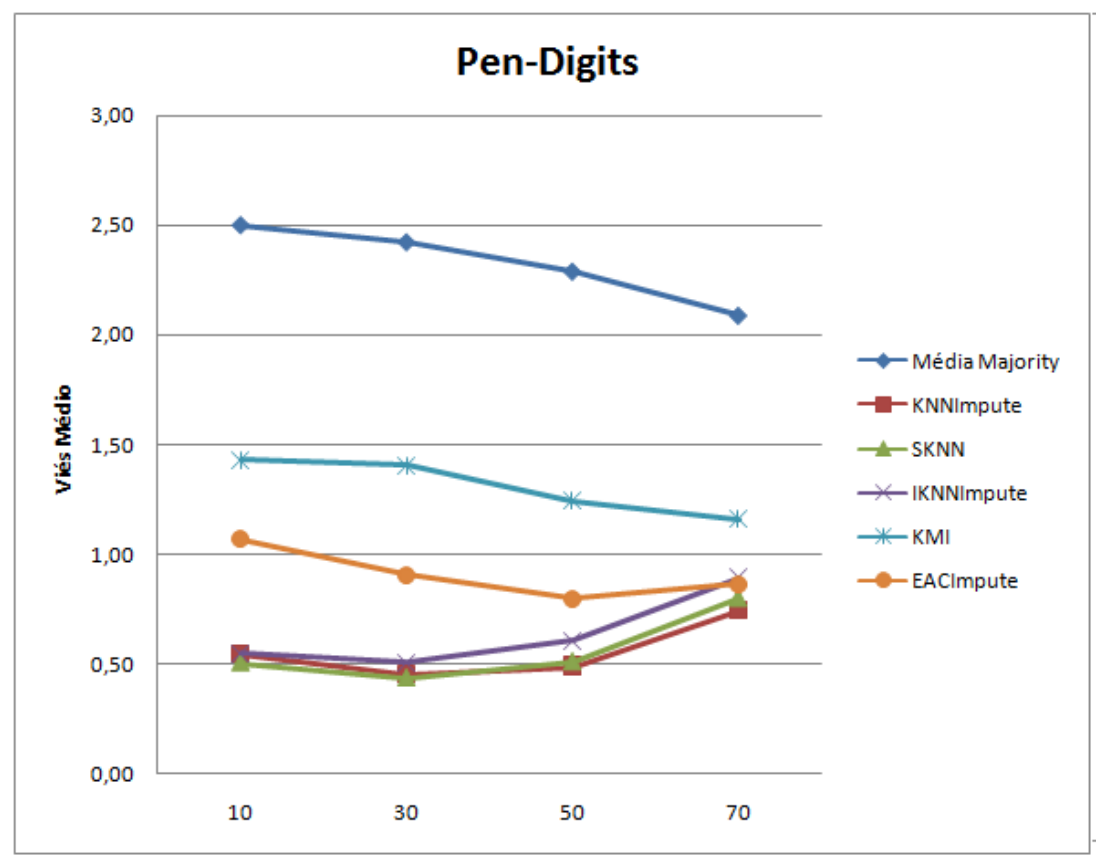

(b)

Figura 4.15: Avaliação por meio da estimação do viés na classificação para a taxa de ausentes em $10 \%, 30 \%, 50 \%$ e $70 \%$. 


\begin{tabular}{|c|c|c|c|c|c|c|c|c|}
\hline $\begin{array}{c}\text { Taxa de } \\
\text { Ausentes }\end{array}$ & $\begin{array}{c}\text { Atributos } \\
\text { Ausentes }\left(q_{a}\right)\end{array}$ & Média Geral & Média Majority & KNNImpute & SKNN & IKNNImpute & KMI & EACImpute \\
\hline \multirow{8}{*}{$10 \%$} & 1 & 7,00 & 4,23 & 3,27 & 3,35 & 3,82 & 3,32 & 3,02 \\
\hline & 2 & 7,00 & 5,63 & 2,63 & 2,67 & 2,90 & 4,00 & 3,17 \\
\hline & 3 & 7,00 & 5,83 & 2,75 & 2,30 & 2,32 & 4,33 & 3,47 \\
\hline & 4 & 7,00 & 6,00 & 2,30 & 2,17 & 2,33 & 4,78 & 3,42 \\
\hline & 5 & 7,00 & 5,97 & 2,30 & 1,93 & 2,13 & 4,70 & 3,97 \\
\hline & 6 & 7,00 & 6,00 & 2,23 & 2,17 & 2,63 & 4,17 & 3,80 \\
\hline & 7 & 7,00 & 6,00 & 2,17 & 2,17 & 2,73 & 4,70 & 3,23 \\
\hline & 8 & 7,00 & 6,00 & 1,97 & 2,20 & 3,10 & 4,33 & 3,40 \\
\hline \multirow{8}{*}{$30 \%$} & 1 & 7,00 & 4,53 & 2,93 & 3,32 & 3,67 & 3,37 & 3,18 \\
\hline & 2 & 7,00 & 5,77 & 2,40 & 2,33 & 2,77 & 4,33 & 3,40 \\
\hline & 3 & 7,00 & 5,90 & 2,03 & 2,20 & 2,77 & 4,65 & 3,45 \\
\hline & 4 & 7,00 & 6,00 & 2,00 & 2,50 & 3,03 & 4,57 & 2,90 \\
\hline & 5 & 7,00 & 5,90 & 2,10 & 2,10 & 2,83 & 4,60 & 3,47 \\
\hline & 6 & 7,00 & 6,00 & 1,93 & 2,27 & 2,80 & 4,57 & 3,43 \\
\hline & 7 & 7,00 & 5,97 & 1,80 & 2,13 & 3,03 & 4,53 & 3,53 \\
\hline & 8 & 7,00 & 6,00 & 2,47 & $\mathbf{1 , 9 3}$ & 2,50 & 4,80 & 3,30 \\
\hline \multirow{8}{*}{$50 \%$} & 1 & 7,00 & 4,93 & 2,87 & 3,27 & 3,60 & 3,43 & 2,90 \\
\hline & 2 & 7,00 & 5,73 & 2,23 & 2,87 & 3,60 & 4,13 & 2,43 \\
\hline & 3 & 7,00 & 6,00 & 2,10 & 2,60 & 3,10 & 4,40 & 2,80 \\
\hline & 4 & 7,00 & 5,93 & 2,13 & 2,33 & 2,90 & 4,43 & 3,27 \\
\hline & 5 & 7,00 & 5,97 & 1,90 & 2,32 & 3,15 & 4,47 & 3,20 \\
\hline & 6 & 7,00 & 5,97 & 1,80 & 2,53 & 3,47 & 4,23 & 3,00 \\
\hline & 7 & 7,00 & 5,97 & $\mathbf{1 , 8 7}$ & 2,43 & 3,57 & 4,13 & 3.03 \\
\hline & 8 & 7,00 & 6,00 & 2,65 & 1,77 & 2,83 & 4,63 & 3,12 \\
\hline \multirow{8}{*}{$70 \%$} & 1 & 7,00 & 3,93 & 3,15 & 3,62 & 4,20 & 3,17 & 2,93 \\
\hline & 2 & 7,00 & 4,80 & 3,03 & 2,97 & 3,10 & 3,73 & 3,37 \\
\hline & 3 & 7,00 & 5,50 & 2,73 & 2,77 & 3,13 & 3,97 & 2,90 \\
\hline & 4 & 7,00 & 5,87 & 2,50 & 2,67 & 3,20 & 4,07 & 2,70 \\
\hline & 5 & 7,00 & 5,23 & 2,47 & 3,50 & 4,40 & 3,17 & 2,23 \\
\hline & 6 & 7,00 & 5,53 & 2,47 & 3,10 & 3,97 & 3,47 & 2,47 \\
\hline & 7 & 7,00 & 5,20 & 2,37 & 3,53 & 4,50 & 3,03 & 2,37 \\
\hline & 8 & 7,00 & 5,80 & 2,53 & 2,43 & 3,23 & 3,97 & 3,03 \\
\hline $\begin{array}{c}\text { Frequência } \\
\text { dos menores } \\
\text { "ranks" }\end{array}$ & - & 0 & 0 & 20 & 11 & 0 & 0 & 5 \\
\hline
\end{tabular}

Tabela 4.24: "Ranks" médios em cada cenário de ausência - estimação do viés na classificação para a base de dados Pen-Digits. Os menores valores de "rank" médios estão destacados em negrito.

\begin{tabular}{|c|c|c|c|c|}
\hline Algoritmo & $10 \%$ & $30 \%$ & $50 \%$ & $70 \%$ \\
\hline Média Geral & $(0 / 0 / 48)$ & $(0 / 0 / 48)$ & $(0 / 0 / 48)$ & $(0 / 0 / 48)$ \\
\hline Média Majority & $(8 / 0 / 40)$ & $(8 / 0 / 40)$ & $(8 / 0 / 40)$ & $(9 / 0 / 39)$ \\
\hline KNNImpute & $(40 / 1 / 7)$ & $(45 / 1 / 2)$ & $(47 / 0 / 1)$ & $(42 / 2 / 4)$ \\
\hline SKNN & $(42 / 1 / 5)$ & $(41 / 1 / 6)$ & $(39 / 0 / 9)$ & $(35 / 0 / 13)$ \\
\hline IKNNImpute & $(32 / 0 / 16)$ & $(29 / 0 / 19)$ & $(26 / 0 / 22)$ & $(20 / 0 / 28)$ \\
\hline KMI & $(18 / 0 / 30)$ & $(17 / 0 / 31)$ & $(17 / 0 / 31)$ & $(23 / 0 / 25)$ \\
\hline EACImpute & $(27 / 0 / 21)$ & $(27 / 0 / 21)$ & $(31 / 0 / 17)$ & $(37 / 2 / 9)$ \\
\hline
\end{tabular}

Tabela 4.25: Total de vitórias, empates e derrotas, em relação aos "ranks" médios relativos à estimação do viés na classificação para a base de dados Pen-Digits. 


\begin{tabular}{|c|c|c|c|c|}
\hline Algoritmos & $10 \%$ & $30 \%$ & $50 \%$ & $70 \%$ \\
\hline Média Geral & $(0 / 7 / 41)$ & $(0 / 7 / 41)$ & $(0 / 7 / 41)$ & $(0 / 4 / 44)$ \\
\hline Média Majority & $(1 / 16 / 31)$ & $(1 / 18 / 29)$ & $(1 / 12 / 35)$ & $(4 / 13 / 31)$ \\
\hline KNNImpute & $(23 / 25 / 0)$ & $(23 / 25 / 0)$ & $(25 / 23 / 0)$ & $(17 / 31 / 0)$ \\
\hline SKNN & $(23 / 25 / 0)$ & $(23 / 25 / 0)$ & $(22 / 26 / 0)$ & $(15 / 33 / 0)$ \\
\hline IKNNImpute & $(21 / 27 / 0)$ & $(22 / 26 / 0)$ & $(17 / 29 / 2)$ & $(13 / 30 / 5)$ \\
\hline KMI & $(11 / 19 / 18)$ & $(9 / 16 / 23)$ & $(12 / 18 / 18)$ & $(15 / 33 / 0)$ \\
\hline EACImpute & $(16 / 27 / 5)$ & $(17 / 29 / 2)$ & $(19 / 29 / 0)$ & $(16 / 32 / 0)$ \\
\hline
\end{tabular}

Tabela 4.26: Total de vitórias, empates e derrotas de cada algoritmo que houve diferença estatística na comparação pareada em cada cenário de ausência - estimação do viés na classificação para a base de dados Pen-Digits.

apresentaram os melhores resultados nas taxas de $10 \%$ e $30 \%$ de valores ausentes. Nas taxas de $50 \%$ e $70 \%$ de valores ausentes, o algoritmo KNNImpute obteve os melhores resultados.

Em resumo, considerando os resultados obtidos com o teste estatístico de Nemenyi para a medida de avaliação por meio do $\overline{E Q_{N}}$ e da estimação do viés na classificação, pode-se afirmar que os melhores resultados foram alcançados pelos algoritmos IKNNImpute e KNNImpute respectivamente. Dessa maneira, sugerindo que melhores resultados na predição não levam, necessariamente, a um menor viés de classificação.

\subsubsection{Base de dados "Segmentation"}

Os resultados referentes aos valores médios da predição $\left(\overline{E Q_{N}}\right)$ nas taxas de $10 \%, 30 \%$, $50 \%$ e $70 \%$ de valores ausentes são apresentados na Figura 4.16. Pode-se perceber que todos os algoritmos apresentaram comportamentos semelhantes, com destaque para os algoritmos $\mathrm{KN}$ NImpute, IKNNImpute e SKNN que obtiveram os melhores resultados. A Tabela 4.27 reporta os valores médios dos "ranks" para cada quantidade de atributos com valores ausentes variando entre 1 e 9. Nesta tabela, pode-se verificar que os melhores resultados foram obtidos pelos algoritmos IKNNImpute, KNNImpute e SKNN, respectivamente. Na Tabela 4.28 são apresentados os resultados do somatório do número de vitórias, empates e derrotas, na comparação pareada dos valores médios dos "ranks". Nesta tabela, o algoritmo IKNNImpute apresentou os melhores resultados em todas as taxas de valores ausentes (empatando apenas com algoritmo KNNImpute na taxa de $10 \%$ de valores ausentes). Resultados detalhados referentes ao número de vitórias, empates e derrotas dos algoritmos de imputação são apresentados na Tabela A.17 do Apêndice.

Diferenças estatísticas em relação aos desempenhos dos algoritmos identificadas por meio do teste de Nemenyi são apresentadas na Tabela A.18 do Apêndice. A Tabela 4.29 reporta os resultados referentes ao somatório do número de vitórias, empates e derrotas de cada algoritmo apresentado na Tabela A.18. Na Tabela 4.29, observa-se que para as taxas de $10 \%$ e $30 \%$ de valores ausentes os algoritmos KNNImpute, SKNN e IKNNImpute apresentaram melhores resultados, obtendo o maior número de vitórias. Nas demais taxas de valores ausentes os algoritmos KNNImpute e IKNNImpute apresentam resultados competitivos, porém, com uma pequena vantagem para o IKNNImpute para a taxa de $70 \%$ de valores ausentes. 


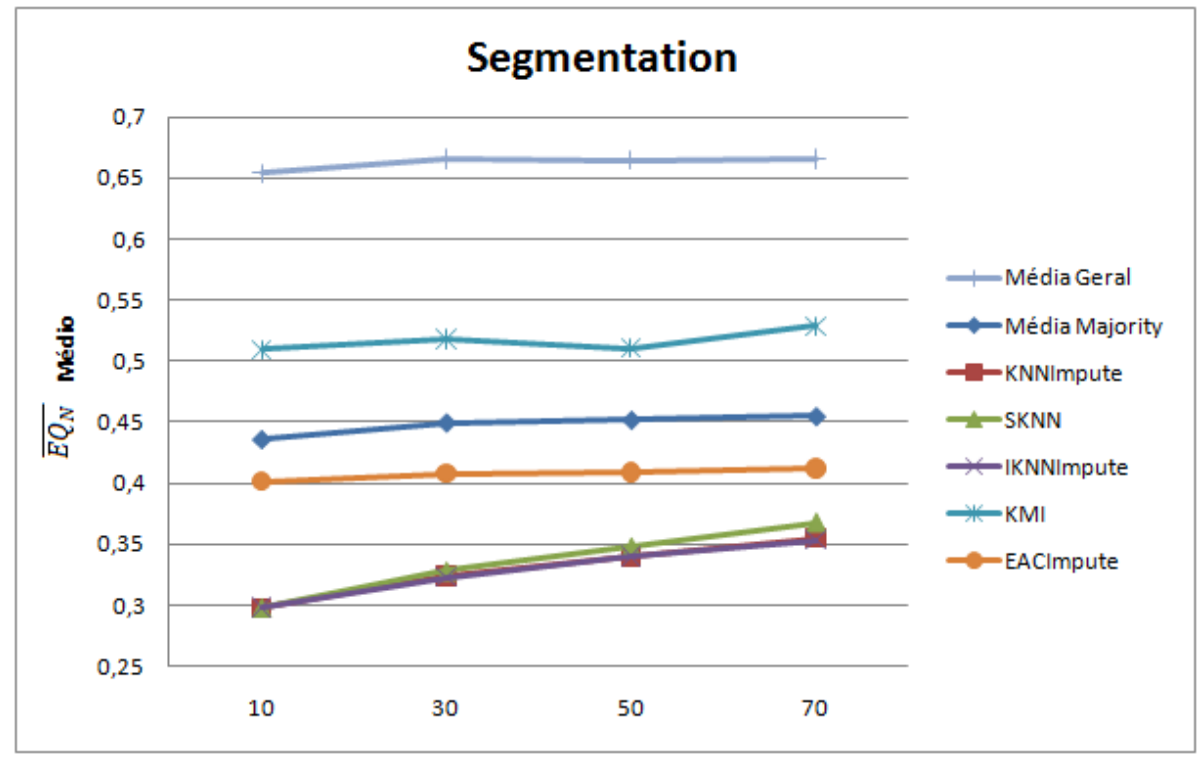

Figura 4.16: Avaliação pelo $\overline{E Q_{N}}$ para a taxa de ausentes em $10 \%, 30 \%, 50 \%$ e $70 \%$.

\begin{tabular}{|c|c|c|c|c|c|c|c|c|}
\hline $\begin{array}{c}\text { Taxa de } \\
\text { Ausentes }\end{array}$ & $\begin{array}{l}\text { Atributos } \\
\text { Ausentes }\end{array}$ & Média Geral & Média Majority & KNNImpute & SKNN & IKNNImpute & KMI & EACImpute \\
\hline \multirow{9}{*}{$10 \%$} & 1 & 6,83 & 5,00 & 2,03 & 2,17 & 2,10 & 5,90 & 3,97 \\
\hline & 2 & 6,93 & 4,87 & 2,03 & 2,00 & 2,00 & 5,87 & 4,30 \\
\hline & 3 & 6,70 & 4,73 & 2,03 & 1,97 & 2,07 & 6,23 & 4,27 \\
\hline & 4 & 6,83 & 4,83 & 1,93 & 2,13 & 1,93 & 6,13 & 4,20 \\
\hline & 5 & 6,87 & 4,77 & 1,70 & 2,17 & 2,13 & 6,00 & 4,37 \\
\hline & 6 & 6,83 & 4,83 & 1,83 & 2,10 & 2,07 & 6,13 & 4,20 \\
\hline & 7 & 6,93 & 4,93 & 1,90 & 2,23 & 1,87 & 5,93 & 4,20 \\
\hline & 8 & 6,77 & 4,83 & 2,10 & 1,87 & 2,03 & 6,13 & 4,27 \\
\hline & 9 & 6,90 & 4,87 & 2,27 & 1,83 & 1,90 & 5,97 & 4,27 \\
\hline \multirow{9}{*}{$30 \%$} & 1 & 6,80 & 4,83 & 2,20 & 2,30 & $\mathbf{1 , 5 0}$ & 6,07 & 4,30 \\
\hline & 2 & 6,87 & 4,83 & 1,87 & 2,73 & 1,43 & 6,03 & 4,23 \\
\hline & 3 & 6,87 & 4,93 & 1,73 & 2,57 & 1,70 & 6,07 & 4,13 \\
\hline & 4 & 6,87 & 4,97 & 1,80 & 2,40 & 1,80 & 6,10 & 4,07 \\
\hline & 5 & 6,93 & 5,00 & 1,87 & 2,43 & 1,70 & 6,07 & 4,00 \\
\hline & 6 & 6,97 & 5,00 & 1,80 & 2,43 & 1,77 & 6,03 & 4,00 \\
\hline & 7 & 6,97 & 4,97 & 1,73 & 2,33 & 1,93 & 6,03 & 4,03 \\
\hline & 8 & 6,90 & 5,00 & $1, \mathbf{8 3}$ & 2,30 & 1,87 & 6,10 & 4,00 \\
\hline & 9 & 6,97 & 5,00 & 2,23 & 2,00 & 1,77 & 6,03 & 4,00 \\
\hline \multirow{9}{*}{$50 \%$} & 1 & 6,87 & 4,90 & 2,23 & 2,33 & 1,43 & 6,07 & 4,17 \\
\hline & 2 & 6,93 & 4,90 & 2,20 & 2,47 & 1,37 & 6,03 & 4,10 \\
\hline & 3 & 6,87 & 4,97 & 1,67 & 2,77 & 1,67 & 6,13 & 3,93 \\
\hline & 4 & 6,90 & 5,00 & 1,67 & 2,77 & $\mathbf{1 , 5 7}$ & 6,10 & 4,00 \\
\hline & 5 & 6,90 & 4,97 & 1,73 & 2,70 & 1,57 & 6,10 & 4,03 \\
\hline & 6 & 6,97 & 4,97 & 1,50 & 2,70 & 1,80 & 6,03 & 4,03 \\
\hline & 7 & 7,00 & 5,00 & 1,40 & 2,63 & 1,97 & 6,00 & 4,00 \\
\hline & 8 & 6,97 & 4,97 & $\mathbf{1 , 5 0}$ & 2,53 & 1,97 & 6,03 & 4,03 \\
\hline & 9 & 6,93 & 5,00 & 2,47 & 2,13 & 1,40 & 6,07 & 4,00 \\
\hline \multirow{9}{*}{$70 \%$} & 1 & 6,87 & 4,93 & 2,37 & 2,73 & 1,07 & 6,13 & 3,90 \\
\hline & 2 & 6,93 & 5,00 & 2,23 & 2,60 & 1,17 & 6,03 & 4,03 \\
\hline & 3 & 6,87 & 5,00 & 1,77 & 2,87 & 1,37 & 6,13 & 4,00 \\
\hline & 4 & 7,00 & 5,00 & 1,63 & 2,83 & 1,53 & 6,00 & 4,00 \\
\hline & 5 & 6,90 & 5,00 & 1,57 & 2,97 & 1,47 & 6,10 & 4,00 \\
\hline & 6 & 6,93 & 5,00 & 1,30 & 2,97 & 1,73 & 6,07 & 4,00 \\
\hline & 7 & 6,90 & 5,00 & 1,30 & 3,00 & 1,70 & 6,10 & 4,00 \\
\hline & 8 & 7,00 & 5,00 & 1,20 & 2,83 & 1,97 & 6,00 & 4,00 \\
\hline & 9 & 7,00 & 5,00 & 1,20 & 2,83 & 1,97 & 6,00 & 4,00 \\
\hline $\begin{array}{c}\text { Frequência } \\
\text { dos menores } \\
\text { "ranks" }\end{array}$ & - & 0 & 0 & 15 & 4 & 21 & 0 & 0 \\
\hline
\end{tabular}

Tabela 4.27: "Ranks" médios em cada cenário de ausência $-\overline{E Q_{N}}$ para a base de dados Segmentation. Os menores valores de "rank" médios estão destacados em negrito. 


\begin{tabular}{|c|c|c|c|c|}
\hline Algoritmo & $10 \%$ & $30 \%$ & $50 \%$ & $70 \%$ \\
\hline Média Geral & $(0 / 0 / 54)$ & $(0 / 0 / 54)$ & $(0 / 0 / 54)$ & $(0 / 0 / 54)$ \\
\hline Média Majority & $(18 / 0 / 36)$ & $(18 / 0 / 36)$ & $(18 / 0 / 36)$ & $(18 / 0 / 36)$ \\
\hline KNNImpute & $(45 / 1 / 8)$ & $(46 / 1 / 7)$ & $(47 / 1 / 6)$ & $(47 / 0 / 7)$ \\
\hline SKNN & $(43 / 1 / 10)$ & $(37 / 0 / 17)$ & $(37 / 0 / 17)$ & $(37 / 0 / 17)$ \\
\hline IKNNImpute & $(45 / 2 / 7)$ & $(51 / 1 / 2)$ & $(50 / 1 / 3)$ & $(51 / 0 / 3)$ \\
\hline KMI & $(8 / 0 / 40)$ & $(9 / 0 / 45)$ & $(9 / 0 / 45)$ & $(9 / 0 / 45)$ \\
\hline EACImpute & $(27 / 0 / 27)$ & $(27 / 0 / 27)$ & $(27 / 0 / 27)$ & $(27 / 0 / 27)$ \\
\hline
\end{tabular}

Tabela 4.28: Total de vitórias, empates e derrotas, em relação aos "ranks" médios $-\overline{E Q_{N}}$ para a base de dados Segmentation.

\begin{tabular}{|c|c|c|c|c|}
\hline Algoritmo & $10 \%$ & $30 \%$ & $50 \%$ & $70 \%$ \\
\hline Média Geral & $(0 / 9 / 45)$ & $(0 / 9 / 45)$ & $(0 / 9 / 45)$ & $(0 / 9 / 45)$ \\
\hline Média Majority & $(10 / 17 / 27)$ & $(9 / 18 / 27)$ & $(9 / 18 / 27)$ & $(9 / 18 / 27)$ \\
\hline KNNImpute & $(36 / 18 / 0)$ & $(36 / 18 / 0)$ & $(36 / 18 / 0)$ & $(40 / 14 / 0)$ \\
\hline SKNN & $(36 / 18 / 0)$ & $(36 / 18 / 0)$ & $(32 / 22 / 0)$ & $(29 / 18 / 7)$ \\
\hline IKNNImpute & $(36 / 18 / 0)$ & $(36 / 18 / 0)$ & $(36 / 18 / 0)$ & $(39 / 15 / 0)$ \\
\hline KMI & $(0 / 17 / 37)$ & $(0 / 18 / 36)$ & $(0 / 18 / 36)$ & $(0 / 18 / 36)$ \\
\hline EACImpute & $(18 / 9 / 27)$ & $(18 / 9 / 27)$ & $(18 / 13 / 23)$ & $(18 / 16 / 20)$ \\
\hline
\end{tabular}

Tabela 4.29: Total de vitórias, empates e derrotas por meio das comparações pareadas de cada algoritmo que houve diferenças estatísticas em cada cenário de ausência $-\overline{E Q_{N}}$ para a base de dados Segmentation.

A Figura 4.17 apresenta os resultados médios da estimação do viés na classificação. Estes resultados foram obtidos por meio do algoritmo de classificação $k$-NN que obteve uma taxa de classificação correta média superior aos demais (ver Tabela 4.2). Observa-se que na Figura 4.17(a), o algoritmo Média Geral apresentou piores resultados em relação aos demais algoritmos. Para uma melhor visualização dos resultados, a Figura 4.17(b) apresenta os resultados dos algoritmos, exceto para o algoritmo Média Geral. Nestes resultados, todos os algoritmos apresentam mesmo comportamento, com destaque para os algoritmos SKNN e IKNNImpute, que apresentaram melhores resultados.

A Tabela 4.30 apresenta os valores médios dos "ranks" obtidos pela avaliação do viés na classificação, na qual pode se observar que o algoritmo IKNNImpute apresenta melhores resultados. A Tabela 4.31 apresenta os resultados referentes aos somatórios do número de vitórias, empates e derrotas em relação aos valores médios dos "ranks" por meio das comparações pareadas. Nesta tabela, o algoritmo IKNNImpute obteve melhores resultados em todas as taxas de valores ausentes.

Analisando os resultados obtidos pelo teste post-hoc de Nemenyi, foram observadas diferenças estatísticas entre vários pares de algoritmos, conforme apresentados na Tabela A.20 do Apêndice. Na Tabela 4.32 são apresentados os resultados totais do número de vitórias, empates e derrotas nas comparações pareadas dos algoritmos que apresentaram diferenças estatísticas. Nesses casos, o algoritmo IKNNImpute apresentou os melhores resultados em todas as taxas de valores ausentes.

Em resumo, considerando os resultados obtidos para o $\overline{E Q_{N}}$ com o teste de Nemenyi, observa-se que os algoritmos KNNImpute e IKNNImpute apresentaram resultados competitivos em nas taxas de 10\%, 30\% e 50\% de valores ausentes, com um destaque para o algoritmo 


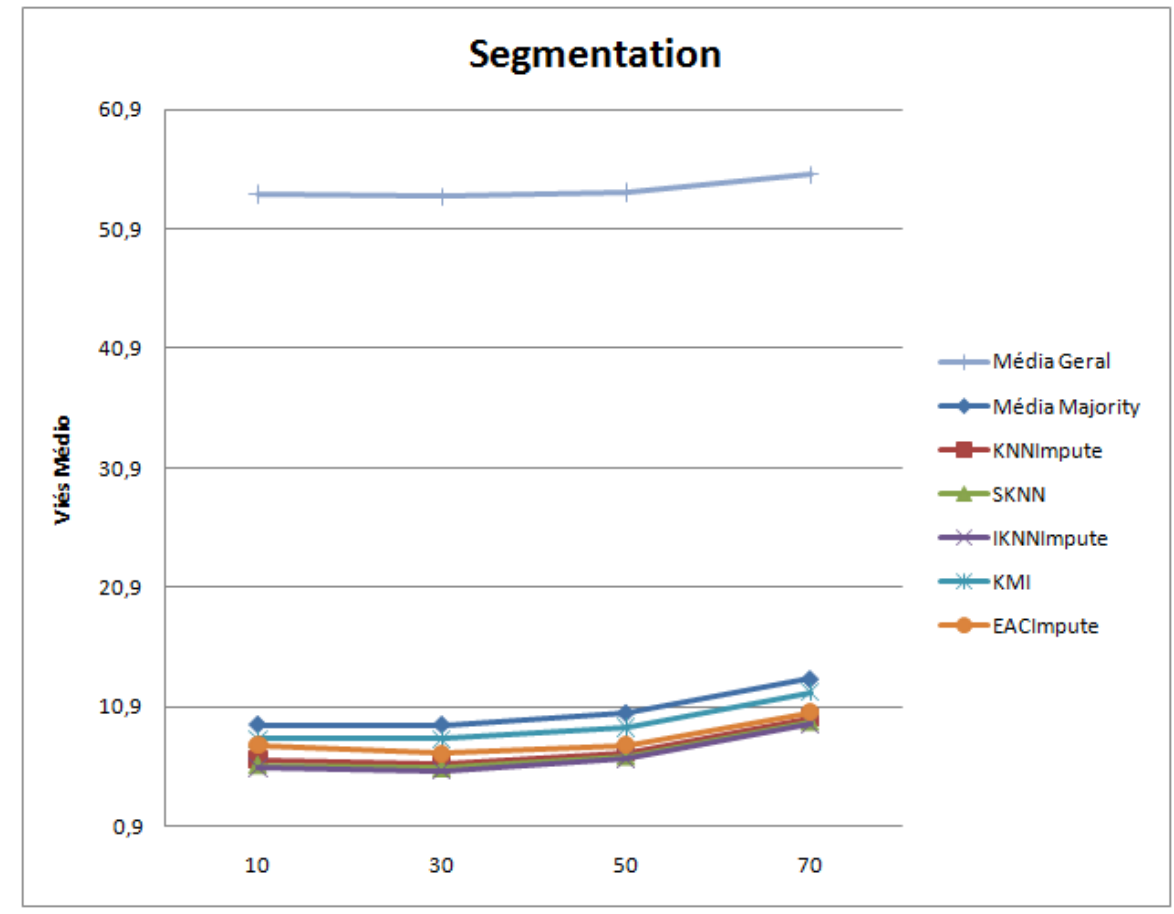

(a)

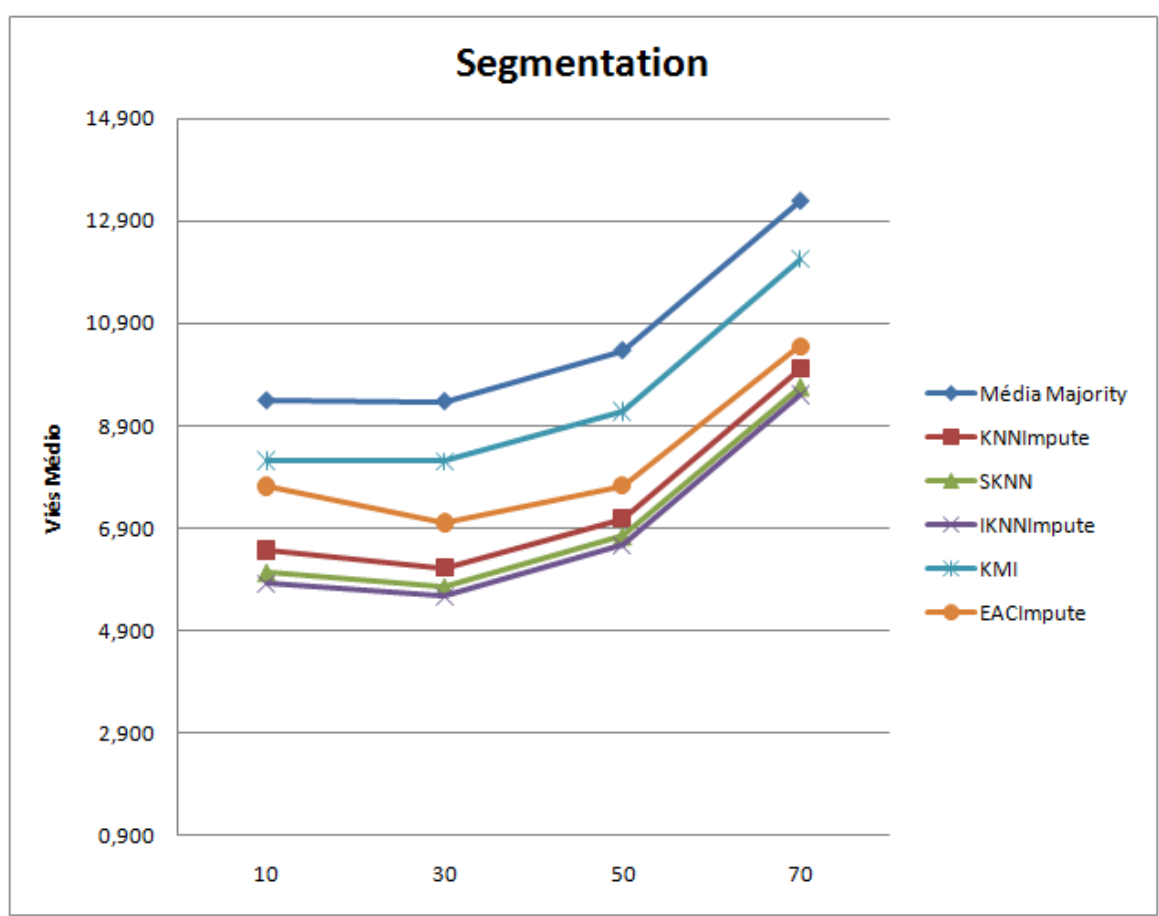

(b)

Figura 4.17: Avaliação pelo bias de classificação para a taxa de ausentes em 10\%, 30\%, 50\% e $70 \%$. 


\begin{tabular}{|c|c|c|c|c|c|c|c|c|}
\hline $\begin{array}{c}\text { Taxa de } \\
\text { Ausentes }\end{array}$ & $\begin{array}{l}\text { Atributos } \\
\text { Ausentes }\end{array}$ & Média Geral & Média Majority & KNNImpute & SKNN & IKNNImpute & KMI & EACImpute \\
\hline \multirow{9}{*}{$10 \%$} & 1 & 7,00 & 5,48 & 3,12 & 2,35 & 1,67 & 4,23 & 4,15 \\
\hline & 2 & 7,00 & 5,77 & 2,80 & 2,05 & 1,60 & 4,70 & 4,08 \\
\hline & 3 & 7,00 & 5,82 & 3,00 & 2,13 & 1,28 & 4,67 & 4,10 \\
\hline & 4 & 7,00 & 5,87 & 2,95 & 2,00 & 1,27 & 4,57 & 4,35 \\
\hline & 5 & 7,00 & 5,83 & 2,75 & 2,17 & 1,40 & 4,63 & 4,22 \\
\hline & 6 & 7,00 & 5,88 & 2,88 & 1,95 & 1,38 & 4,52 & 4,38 \\
\hline & 7 & 7,00 & 5,88 & 2,85 & 2,02 & 1,32 & 4,53 & 4,40 \\
\hline & 8 & 7,00 & 5,93 & 3,02 & 2,05 & 1,17 & 4,60 & 4,23 \\
\hline & 9 & 7,00 & 5,97 & 3,02 & 2,05 & 1,17 & 4,70 & 4,10 \\
\hline \multirow{9}{*}{$30 \%$} & 1 & 7,00 & 5,77 & 2,82 & 2,03 & 1,25 & 4,80 & 4,33 \\
\hline & 2 & 7,00 & 5,83 & 3,00 & 1,90 & 1,15 & 4,75 & 4,37 \\
\hline & 3 & 7,00 & 5,97 & 3,02 & 1,90 & 1,12 & 4,77 & 4,23 \\
\hline & 4 & 7,00 & 5,93 & 2,95 & 2,00 & 1,13 & 4,70 & 4,28 \\
\hline & 5 & 7,00 & 6,00 & 2,87 & 1,92 & 1,22 & 4,82 & 4,18 \\
\hline & 6 & 7,00 & 6,00 & 2,85 & 2,02 & 1,30 & 4,78 & 4,05 \\
\hline & 7 & 7,00 & 6,00 & 2,88 & 2,05 & 1,25 & 4,90 & 3,92 \\
\hline & 8 & 7,00 & 6,00 & 3,00 & 2,13 & 1,13 & 4,93 & 3,80 \\
\hline & 9 & 7,00 & 6,00 & 2,93 & 2,12 & 1,22 & 5,00 & 3,73 \\
\hline \multirow{9}{*}{$50 \%$} & 1 & 7,00 & 5,67 & 2,88 & 1,92 & 1,52 & 4,98 & 4,03 \\
\hline & 2 & 7,00 & 5,87 & 2,88 & 2,08 & 1,13 & 4,73 & 4,30 \\
\hline & 3 & 7,00 & 5,93 & 3,05 & 1,85 & 1,33 & 4,87 & 3,97 \\
\hline & 4 & 7,00 & 6,00 & 2,87 & 2,08 & 1,15 & 5,00 & 3,90 \\
\hline & 5 & 7,00 & 6,00 & 2,90 & 2,03 & 1,30 & 5,00 & 3,77 \\
\hline & 6 & 7,00 & 6,00 & 3,08 & 2,02 & 1,10 & 4,93 & 3,87 \\
\hline & 7 & 7,00 & 6,00 & 3,03 & 2,13 & 1,10 & 4,83 & 3,90 \\
\hline & 8 & 7,00 & 6,00 & 3,07 & 2,08 & 1,23 & 4,87 & 3,75 \\
\hline & 9 & 7,00 & 6,00 & 3,35 & 2,27 & 1,25 & 4,97 & 3,17 \\
\hline \multirow{9}{*}{$70 \%$} & 1 & 7,00 & 5,90 & 2,83 & 1,87 & 1,83 & 4,98 & 3,58 \\
\hline & 2 & 7,00 & 6,00 & 2,95 & 1,97 & 1,58 & 4,97 & 3,53 \\
\hline & 3 & 7,00 & 6,00 & 3,03 & 1,83 & 1,20 & 4,87 & 4,07 \\
\hline & 4 & 7,00 & 6,00 & 2,93 & 2,00 & 1,17 & 4,97 & 3,93 \\
\hline & 5 & 7,00 & 6,00 & 2,93 & 2,17 & 1,37 & 4,97 & 3,57 \\
\hline & 6 & 7,00 & 6,00 & 3,13 & 2,13 & 1,23 & 4,87 & 3,63 \\
\hline & 7 & 7,00 & 6,00 & 2,95 & 2,03 & 1,53 & 4,97 & 3,52 \\
\hline & 8 & 7,00 & 6,00 & 3,18 & 2,10 & 1,17 & 4,97 & 3,58 \\
\hline & 9 & 7,00 & 6,00 & 3,43 & 2,18 & 1,27 & 4,93 & 3,18 \\
\hline $\begin{array}{l}\text { Frequência } \\
\text { dos menores } \\
\text { "ranks" }\end{array}$ & - & 0 & 0 & 0 & 0 & 36 & 0 & 0 \\
\hline
\end{tabular}

Tabela 4.30: "Ranks" médios em cada cenário de ausência - estimação do viés na classificação para a base de dados Segmentation. Os menores valores de "rank" médios estão destacados em negrito.

\begin{tabular}{|c|c|c|c|c|}
\hline Algoritmo & $10 \%$ & $30 \%$ & $50 \%$ & $70 \%$ \\
\hline Média Geral & $(0 / 0 / 54)$ & $(0 / 0 / 54)$ & $(0 / 0 / 54)$ & $(0 / 0 / 54)$ \\
\hline Média Majority & $(9 / 0 / 45)$ & $(9 / 0 / 45)$ & $(9 / 0 / 45)$ & $(9 / 0 / 45)$ \\
\hline KNNImpute & $(36 / 0 / 18)$ & $(36 / 0 / 18)$ & $(35 / 0 / 19)$ & $(35 / 0 / 19)$ \\
\hline SKNN & $(45 / 0 / 9)$ & $(45 / 0 / 9)$ & $(45 / 0 / 9)$ & $(45 / 0 / 9)$ \\
\hline IKNNImpute & $(54 / 0 / 0)$ & $(54 / 0 / 0)$ & $(54 / 0 / 0)$ & $(54 / 0 / 0)$ \\
\hline KMI & $(18 / 0 / 36)$ & $(18 / 0 / 36)$ & $(18 / 0 / 36)$ & $(18 / 0 / 36)$ \\
\hline EACImpute & $(27 / 0 / 27)$ & $(27 / 0 / 27)$ & $(28 / 0 / 26)$ & $(28 / 0 / 26)$ \\
\hline
\end{tabular}

Tabela 4.31: Total de vitórias, empates e derrotas, em relação aos "ranks" médio - estimação do viés na classificação para a base de dados Segmentation. 


\begin{tabular}{|c|c|c|c|c|}
\hline Algoritmos & $10 \%$ & $30 \%$ & $50 \%$ & $70 \%$ \\
\hline Média Geral & $(0 / 8 / 46)$ & $(0 / 9 / 45)$ & $(0 / 9 / 45)$ & $(0 / 9 / 45)$ \\
\hline Média Majority & $(1 / 15 / 38)$ & $(0 / 18 / 36)$ & $(0 / 18 / 36)$ & $(0 / 18 / 36)$ \\
\hline KNNImpute & $(30 / 18 / 6)$ & $(29 / 16 / 9)$ & $(28 / 18 / 8)$ & $(27 / 20 / 7)$ \\
\hline SKNN & $(36 / 18 / 0)$ & $(36 / 18 / 0)$ & $(35 / 19 / 0)$ & $(35 / 19 / 0)$ \\
\hline IKNNImpute & $(42 / 12 / 0)$ & $(45 / 9 / 0)$ & $(44 / 10 / 0)$ & $(43 / 11 / 0)$ \\
\hline KMI & $(12 / 16 / 26)$ & $(9 / 18 / 27)$ & $(9 / 17 / 28)$ & $(9 / 14 / 31)$ \\
\hline EACImpute & $(17 / 15 / 22)$ & $(18 / 16 / 20)$ & $(19 / 17 / 18)$ & $(22 / 15 / 17)$ \\
\hline
\end{tabular}

Tabela 4.32: Total de vitórias, empates e derrotas por meio das comparações pareadas entre os algoritmos quando houve diferença estatística em cada cenário de ausência - estimação do viés na classificação para a base de dados Segmentation.

KNNImpute que obteve maior número de vitórias para a taxa de $70 \%$ de valores ausentes. Em relação a tarefa de classificação, o algoritmo IKKNImpute apresentou melhor desempenho em todas as taxas de valores ausentes. Neste caso, pode-se afirmar que existe uma correlação entre os resultados de predição e de inserção de viés, pois o algoritmo IKNNImpute se destacou em ambas as avaliações.

\subsubsection{Aleatório - MAR}

Esta seção apresenta os resultados obtidos pela substituição dos valores ausentes simulados aleatoriamente por meio do mecanismo MAR (Missing at random), para as bases de dados Sintética (Garcia e Hruschka, 2005), Íris e Segmentation, utilizando os algoritmos Média Majority (Kononenko et al., 1984) , KNNImpute (Troyanskaya et al., 2001), SKNN (Kim et al., 2004), IKNNImpute (Brás e Menezes, 2007), KMI (Hruschka et al., 2004) e EACImpute. O algoritmo de imputação Média Geral não foi utilizado neste estudo por ter apresentado resultados inferiores, comparados com os demais algoritmos, no mecanismo MCAR. Cumpre, entretanto, mencionar que uma variação deste algoritmo (Média Majority) apresentou, em alguns cenários, resultados competitivos no mecanismo MCAR e foi, portanto, considerado nos experimentos aqui reportados. No mecanismo MAR, simulações foram executadas com a omissão de valores em apenas um atributo de cada base de dados, a saber: o melhor atributo selecionado por meio do procedimento descrito na Seção 4.2.2. O classificador J4.8 - o mesmo utilizado para a seleção dos atributos (Seção 4.2.2) - foi adotado nos experimentos relacionados à estimação do viés na classificação para todas as bases de dados.

\subsubsection{Base de Dados "Sintética"}

Esta base de dados, proposta em Garcia e Hruschka (2005), foi adotada para ilustrar algumas dificuldades encontradas pelos algoritmos de imputação num cenário em que o mecanismo MAR está presente. Para ilustrar tal cenário, considere que 30\% das instâncias dessa base de dados contém valores ausentes no atributo $y$, conforme abordado na Seção 4.2.2. De maneira formal, a probabilidade $p(y=? \mid x \in[0,2])=0,3$. Para este cenário de ausência, considere também a escolha do algoritmo Média Majority para a tarefa de imputação. Lembrando que a imputação é realizada de maneira supervisionada, o algoritmo Média Majority realiza a 
estimação dos valores ausentes por meio da média dos valores conhecidos no atributo $y$, condicionada à informação da classe. O resultado da aplicação desse algoritmo é apresentado na Figura 4.18. Para efeito de visualização, são apresentadas as instâncias que terão valores omitidos (ausentes) no atributo $y$ - representadas pelo símbolo " $\nabla$ " - (para a classe $C_{1}$ ). Uma linha tracejada mostra o deslocamento do valor imputado (figurado pelo símbolo " ao valor original (o comprimento desta linha reflete o erro da predição). Nesta figura, há dois retângulos que ilustram as instâncias que pertencem à mesma classe (neste caso, a classe $C_{1}$ ). Neste cenário, o algoritmo considera as informações disponíveis nos dois retângulos, destacados na Figura 4.18. Consequentemente, os valores estimados para o atributo $y$ serão baseados nos valores existentes nos dois retângulos, fazendo com que os valores médios estejam entre 3 e 4. Resultados análogos são obtidos ao se imputar valores para as instâncias de classe $C_{2}$, com a diferença de que os valores imputados se situarão na faixa $[2,3]$, conforme apresentada na Figura 4.19.

No que diz respeito aos algoritmos KNNImpute e SKNN, percebe-se que estes apresentaram desempenhos semelhantes (ver Figura 4.19) fazendo com que os valores imputados mantenham as instâncias nas respectivas regiões de suas classes. O algoritmo IKNNImpute foi mais impreciso, para algumas instâncias, do que os algoritmos KNNImpute e SKNN. Isto acontece porque o algoritmo tem um passo inicial de imputação por meio da média e posteriormente recalcula os valores imputados iterativamente baseando-se no método $k$-NN. Já os algoritmos KMI e EACImpute estimaram os valores ausentes fazendo com que os mesmos fiquem em torno do centro de suas respectivas regiões de classes. Isto é compreensível pois estes algoritmos são baseados em agrupamento de dados. Neste caso, o algoritmo KMI obteve bons resultados por haver dois grupos para as classes com valores ausentes e porque seu parâmetro padrão, relativo ao número de grupos, ter sido fixado em 2. Quando não se conhece o número de grupos, em situações extremas o algoritmo pode apresentar resultados indesejáveis semelhantes ao algoritmo Média Majority. O algoritmo EACImpute apresentou resultados semelhantes ao KMI. Porém, o algoritmo EACImpute, durante seu processo de busca evolutiva, encontra automaticamente o número de grupos.

Os resultados referentes à predição $\left(\overline{E Q_{N}}\right)$ para cada algoritmo, para as taxas de $10 \%, 30 \%$, $50 \%$ e $70 \%$ de valores ausentes, podem ser visualizados por meio da Figura 4.20. Nestes resultados, os algoritmos KMI, EACImpute, SKNN, KNNImpute, obtiveram comportamentos semelhantes em todas as taxas, destacando-se com os melhores resultados. Na Tabela 4.33 são apresentados os valores médios dos "ranks", na qual observa-se que o algoritmo KMI obteve melhores resultados. Os valores totais referentes às comparações dos valores médios dos "ranks" entre os algoritmos são apresentados na Tabela 4.34. Nestes resultados, o algoritmo KMI apresentou melhores resultados (menor "rank"), seguido pelo algoritmo EACImpute, que apresentou melhores resultados em relação aos algoritmos Média Majority, KNNImpute, SKNN e IKNNImpute. Resultados detalhados referentes às comparações pareadas dos algoritmos podem ser encontrados na Tabela A.21 do Apêndice. Na Tabela 4.34 são apresentados os resultados 


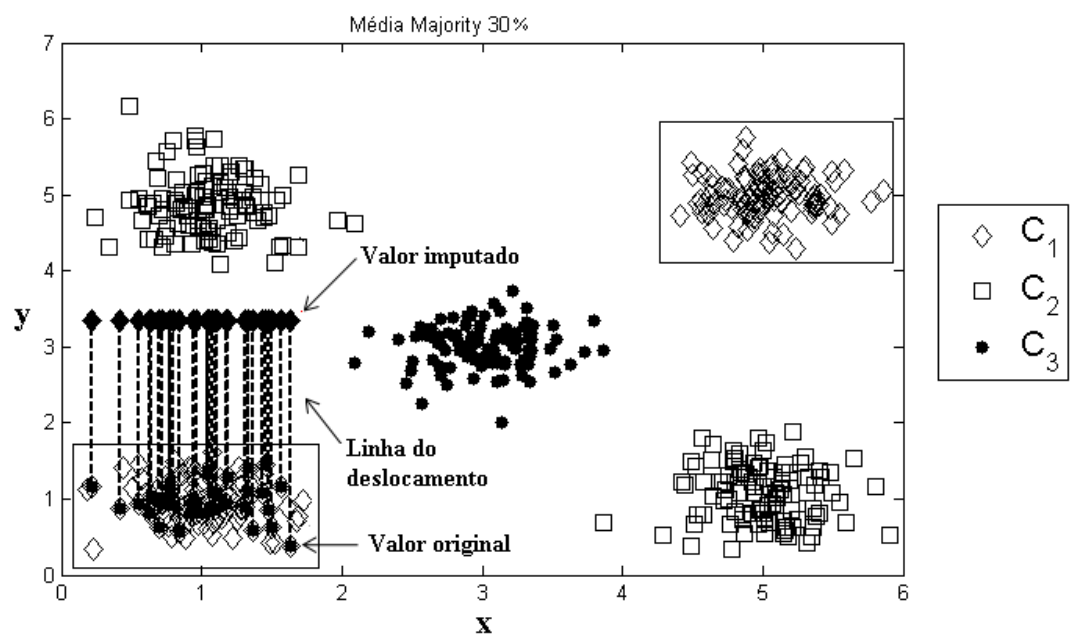

Figura 4.18: Resultado da imputação dos valores ausentes para o algoritmo Média Majority para a taxa de $30 \%$ de valores ausentes.

\begin{tabular}{|c|c|c|c|c|c|c|}
\hline $\begin{array}{c}\text { Taxa de } \\
\text { Ausentes }\end{array}$ & Média Majority & KNNImpute & SKNN & IKNNImpute & KMI & EACImpute \\
\hline $10 \%$ & 6,00 & 3,68 & 3,58 & 3,80 & $\mathbf{1 , 8 3}$ & 2,10 \\
\hline $30 \%$ & 6,00 & 3,50 & 3,50 & 4,87 & $\mathbf{1 , 4 7}$ & 1,67 \\
\hline $50 \%$ & 6,00 & 3,47 & 3,53 & 5,00 & $\mathbf{1 , 4 7}$ & 1,53 \\
\hline $70 \%$ & 6,00 & 3,57 & 3,43 & 5,00 & $\mathbf{1 , 4 7}$ & 1,53 \\
\hline $\begin{array}{c}\text { Frequência } \\
\text { dos menores } \\
\text { "ranks" }\end{array}$ & 0 & 0 & 0 & 0 & 4 & 0 \\
\hline
\end{tabular}

Tabela 4.33: "Ranks" médios em cada cenário de ausência $-\overline{E Q_{N}}$ para a base de dados Sintética. Os menores valores de "rank" médios estão destacados em negrito.

referentes ao somatório do número de vitórias, empates e derrotas de cada algoritmo.

Em relação às análises estatísticas realizada com o teste de Nemenyi foram observadas diferenças estatísticas entre os algoritmos de imputação, conforme apresentadas na Tabela A.22 do Apêndice. Na Tabela 4.35 são apresentados os resultados totais referentes às comparações entre os algoritmos que obtiveram diferenças estatísticas. Nestes resultados, os algoritmos KMI e EACImpute apresentaram os melhores resultados em todas as taxas de valores ausentes.

No que diz respeito à estimação do viés na classificação, na Figura 4.21 são apresentados os resultados médios de cada algoritmo de imputação nas taxas de 10\%, 30\%, 50\% e 70\% de valores ausentes. No gráfico desta figura, o algoritmo Média Majority apresentou resultados inferiores em relação aos demais algoritmos. Estes resultados se devem ao fato de que nesta

\begin{tabular}{|c|c|c|c|c|}
\hline Algoritmo & $10 \%$ & $30 \%$ & $50 \%$ & $70 \%$ \\
\hline Média Majority & $(0 / 0 / 5)$ & $(0 / 0 / 5)$ & $(0 / 0 / 5)$ & $(0 / 0 / 5)$ \\
\hline KNNImpute & $(2 / 0 / 3)$ & $(2 / 1 / 2)$ & $(3 / 0 / 2)$ & $(2 / 0 / 3)$ \\
\hline SKNN & $(3 / 0 / 2)$ & $(2 / 1 / 2)$ & $(2 / 0 / 3)$ & $(3 / 0 / 2)$ \\
\hline IKNNImpute & $(1 / 0 / 4)$ & $(1 / 0 / 4)$ & $(1 / 0 / 4)$ & $(1 / 0 / 4)$ \\
\hline KMI & $(5 / 0 / 0)$ & $(5 / 0 / 0)$ & $(5 / 0 / 0)$ & $(5 / 0 / 0)$ \\
\hline EACImpute & $(4 / 0 / 1)$ & $(4 / 0 / 1)$ & $(4 / 0 / 1)$ & $(4 / 0 / 1)$ \\
\hline
\end{tabular}

Tabela 4.34: Total de vitórias, empates e derrotas, em relação aos "ranks" médios $-\overline{E Q_{N}}$ para a base de dados Sintética 


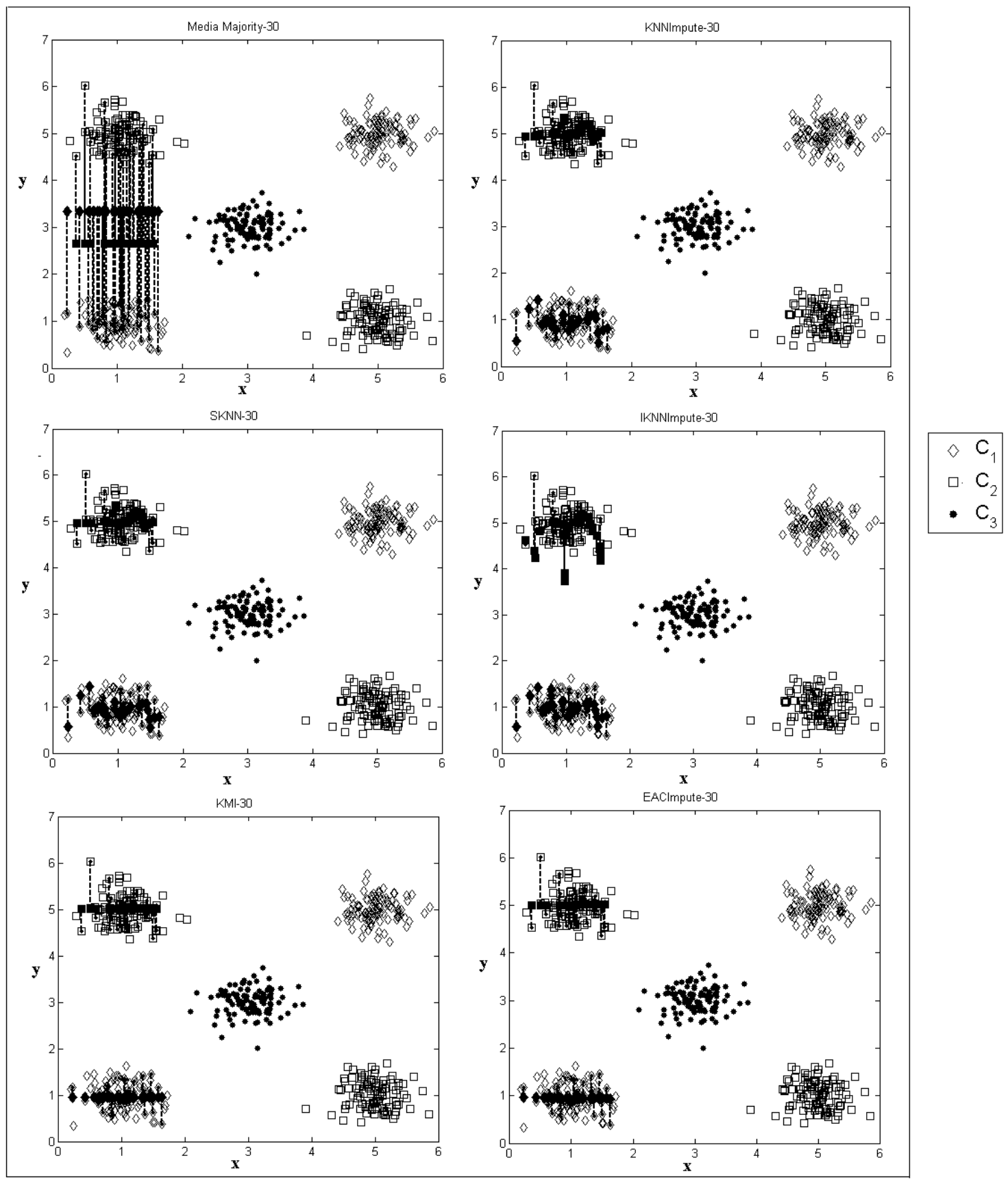

Figura 4.19: Resultado da imputação dos valores ausentes para os algoritmos de imputação (Média Majority, KNNImpute, SKNN, IKNNImpute e EACImpute) na taxa de $30 \%$ de valores ausentes.

\begin{tabular}{|c|c|c|c|c|}
\hline Algoritmo & $10 \%$ & $30 \%$ & $50 \%$ & $70 \%$ \\
\hline Média Majority & $(0 / 0 / 5)$ & $(0 / 1 / 4)$ & $(0 / 1 / 4)$ & $(0 / 1 / 4)$ \\
\hline KNNImpute & $(1 / 2 / 2)$ & $(1 / 2 / 2)$ & $(2 / 1 / 2)$ & $(2 / 1 / 2)$ \\
\hline SKNN & $(1 / 2 / 2)$ & $(1 / 2 / 2)$ & $(2 / 1 / 2)$ & $(2 / 1 / 2)$ \\
\hline IKNNImpute & $(1 / 2 / 2)$ & $(0 / 3 / 2)$ & $(0 / 1 / 4)$ & $(0 / 1 / 4)$ \\
\hline KMI & $(4 / 1 / 0)$ & $(4 / 1 / 0)$ & $(4 / 1 / 0)$ & $(4 / 1 / 0)$ \\
\hline EACImpute & $(4 / 1 / 0)$ & $(4 / 1 / 0)$ & $(4 / 1 / 0)$ & $(4 / 1 / 0)$ \\
\hline
\end{tabular}

Tabela 4.35: Total de vitórias, empates e derrotas, em relação às diferenças estatísticas, de cada algoritmo $-\overline{E Q_{N}}$ para a base de dados Sintética. 


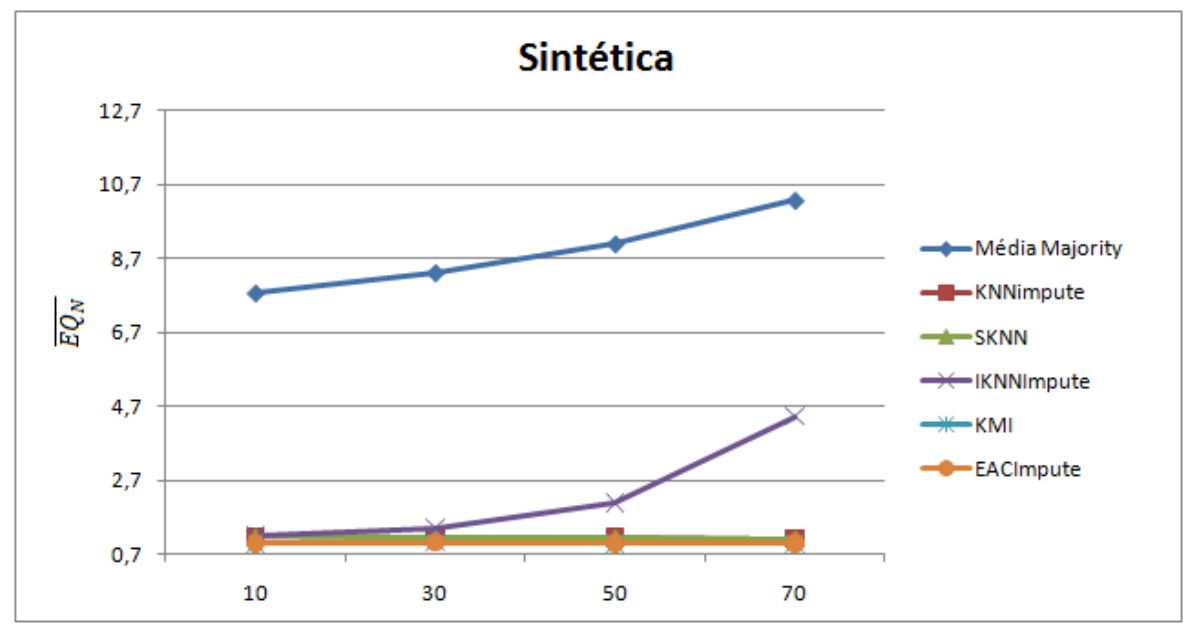

Figura 4.20: Avaliação pelo $\overline{E Q_{N}}$ para a taxa de ausentes em $10 \%, 30 \%$, 50\% e $70 \%$.

medida de avaliação é verificado se os valores estimados pelos algoritmos de imputação ultrapassam uma fronteira de decisão determinada por um algoritmo de classificação. Uma fronteira de decisão se caracteriza por um limitante de uma área ou região contendo as instâncias de uma determinada classe. A Figura 4.22 apresenta uma fronteira de decisão, representada pelos retângulos tracejados, induzida pela árvore de decisão J4.8. Observa-se que nesta figura os valores estimados pelo Média Majority, para a taxa de $30 \%$ de valores ausentes para classe $C_{1}$, se encontram na região da fronteira de decisão da classe $C_{2}$. Dessa forma, as instâncias com valores imputados de $C_{1}$ seriam erroneamente classificadas como pertencentes à classe $C_{2}$. Relembrando que o procedimento de estimação do viés na classificação, apresentada na Seção 4.3, consiste na diferença entre a taxa de classificação correta média dos dados imputados $\left(T C C M_{F}\right)$ e da taxa de classificação correta média dos dados completos $\left(T C C M_{C}\right)$, a diferença absoluta (adotada nos experimentos) da taxa de acerto $T C C M_{C}=100$ e da taxa $T C C M_{C}=0$ resulta no viés de classificação igual a $100 \%$.

A Tabela 4.36 reporta os resultados relativos aos "ranks" médios de cada algoritmo. Esta tabela sugere um equilíbrio de desempenho entre os algoritmos KNNImpute, SKNN, KMI e EACImpute. Na Tabela 4.37 são apresentados os resultados referentes aos totais de vitórias, empates e derrotas de cada algoritmo para cada taxa de valores ausentes. Nestes resultados, todos os algoritmos, exceto o algoritmo Média Majority, apresentam comportamentos semelhantes nas taxas de $10 \%$ e $30 \%$ de valores ausentes. Nas demais taxas de valores ausentes, os algoritmos KNNImpute, SKNN, KMI e EACImpute apresentaram os melhores resultados. Resultados adicionais referentes ao número de vitórias, empates e derrotas das comparações pareadas dos algoritmos podem ser encontradas na Tabela A.23 do Apêndice.

Diferenças estatísticas entre os pares de algoritmos estão reportadas na Tabela A.24 do Apêndice. Estes resultados foram sumarizados por meio do somatório do número de vitórias, empates e derrotas e apresentados na Tabela 4.38. Nesses resultados, os algoritmos apresentam resultados semelhantes. A exceção fica por conta do Média Majority, que se destacou negativa- 


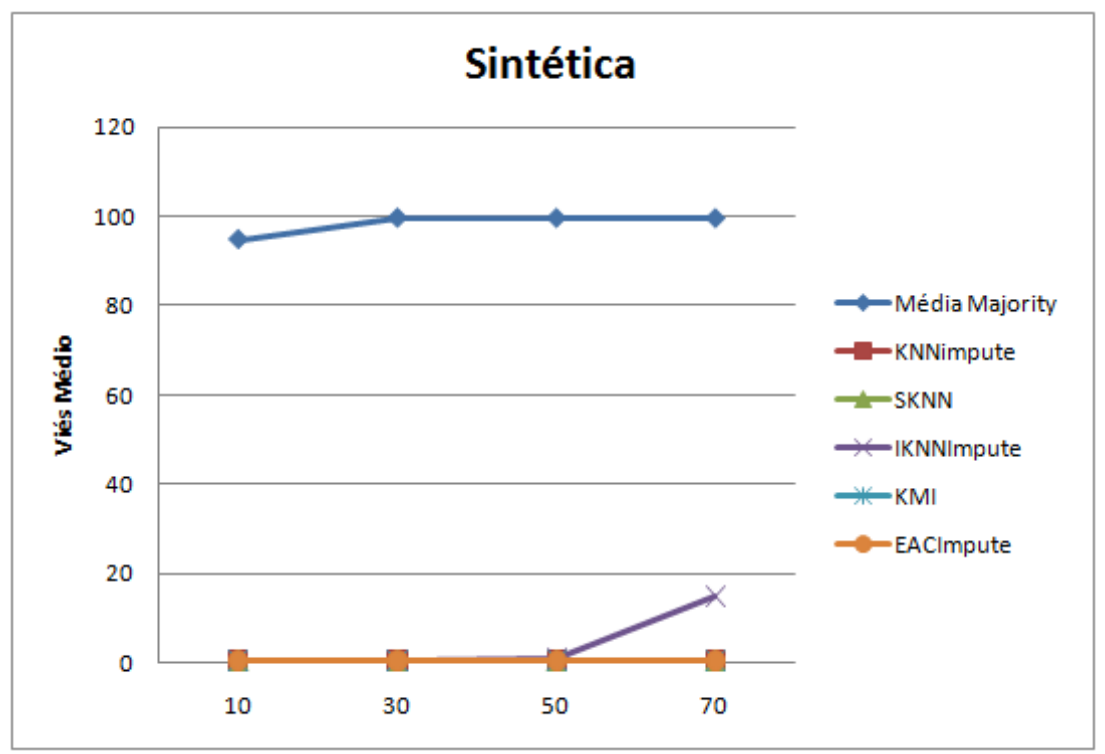

Figura 4.21: Avaliação por meio da estimação do viés na classificação para a base Sintética.
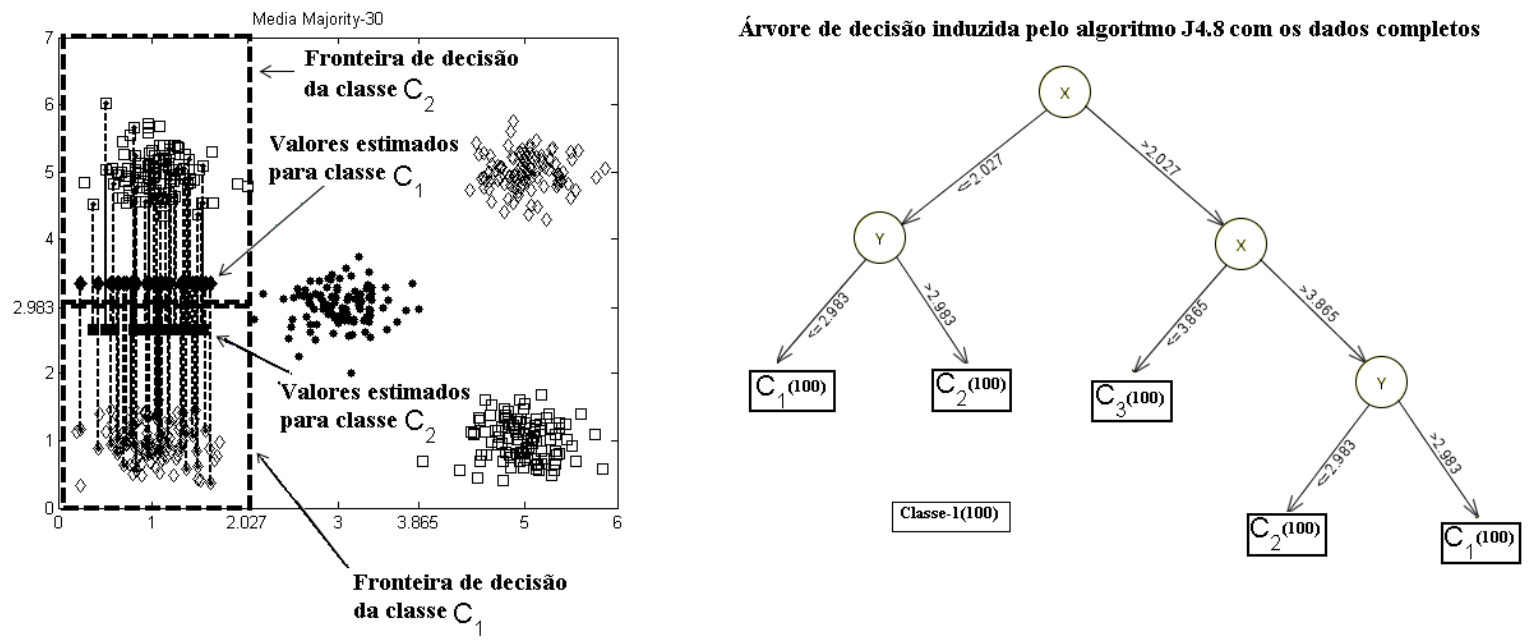

Figura 4.22: Fronteira de decisão (esquerda) construída pelo algoritmo J4.8 (direita) para os valores estimados com o algoritmo Média Majority na taxa de 30\% de valores ausentes.

\begin{tabular}{|c|c|c|c|c|c|c|}
\hline $\begin{array}{c}\text { Taxa de } \\
\text { Ausentes }\end{array}$ & Média Majority & KNNImpute & SKNN & IKNNImpute & KMI & EACImpute \\
\hline $10 \%$ & 6,00 & $\mathbf{3 , 0 0}$ & $\mathbf{3 , 0 0}$ & $\mathbf{3 , 0 0}$ & $\mathbf{3 , 0 0}$ & $\mathbf{3 , 0 0}$ \\
\hline $30 \%$ & 6,00 & $\mathbf{3 , 0 0}$ & $\mathbf{3 , 0 0}$ & $\mathbf{3 , 0 0}$ & $\mathbf{3 , 0 0}$ & $\mathbf{3 , 0 0}$ \\
\hline $50 \%$ & 6,00 & $\mathbf{2 , 8 8}$ & $\mathbf{2 , 8 8}$ & 3,47 & $\mathbf{2 , 8 8}$ & $\mathbf{2 , 8 8}$ \\
\hline $70 \%$ & 6,00 & $\mathbf{2 , 5 0}$ & $\mathbf{2 , 5 0}$ & 5,00 & $\mathbf{2 , 5 0}$ & $\mathbf{2 , 5 0}$ \\
\hline $\begin{array}{c}\text { Frequência } \\
\text { dos menores } \\
\text { "ranks" }\end{array}$ & 0 & 4 & 4 & 2 & 4 & 4 \\
\hline
\end{tabular}

Tabela 4.36: "Ranks" médios em cada cenário de ausência - estimação do viés de classificação para a base de dados Sintética. Os menores valores de "rank" médios estão destacados em negrito. 


\begin{tabular}{|c|c|c|c|c|}
\hline Algoritmo & $10 \%$ & $30 \%$ & $50 \%$ & $70 \%$ \\
\hline Média Majority & $(0 / 0 / 5)$ & $(0 / 0 / 5)$ & $(0 / 0 / 5)$ & $(0 / 0 / 5)$ \\
\hline KNNImpute & $(1 / 4 / 0)$ & $(1 / 4 / 0)$ & $(2 / 3 / 0)$ & $(2 / 3 / 0)$ \\
\hline SKNN & $(1 / 4 / 0)$ & $(1 / 4 / 0)$ & $(2 / 3 / 0)$ & $(2 / 3 / 0)$ \\
\hline IKNNImpute & $(1 / 4 / 0)$ & $(1 / 4 / 0)$ & $(1 / 0 / 4)$ & $(1 / 0 / 4)$ \\
\hline KMI & $(1 / 4 / 0)$ & $(1 / 4 / 0)$ & $(2 / 3 / 0)$ & $(2 / 3 / 0)$ \\
\hline EACImpute & $(1 / 4 / 0)$ & $(1 / 4 / 0)$ & $(2 / 3 / 0)$ & $(2 / 3 / 0)$ \\
\hline
\end{tabular}

Tabela 4.37: Total de vitórias, empates e derrotas, em relação aos "ranks" médios - estimação do viés na classificação para a base de dados Sintética

\begin{tabular}{|c|c|c|c|c|}
\hline Algoritmo & $10 \%$ & $30 \%$ & $50 \%$ & $70 \%$ \\
\hline Média Majority & $(0 / 0 / 5)$ & $(0 / 0 / 5)$ & $(0 / 0 / 5)$ & $(0 / 1 / 4)$ \\
\hline KNNImpute & $(1 / 4 / 0)$ & $(1 / 4 / 0)$ & $(1 / 4 / 0)$ & $(2 / 3 / 0)$ \\
\hline SKNN & $(1 / 4 / 0)$ & $(1 / 4 / 0)$ & $(1 / 4 / 0)$ & $(2 / 3 / 0)$ \\
\hline IKNNImpute & $(1 / 4 / 0)$ & $(1 / 4 / 0)$ & $(1 / 4 / 0)$ & $(0 / 1 / 4)$ \\
\hline KMI & $(1 / 4 / 0)$ & $(1 / 4 / 0)$ & $(1 / 4 / 0)$ & $(2 / 3 / 0)$ \\
\hline EACImpute & $(1 / 4 / 0)$ & $(1 / 4 / 0)$ & $(1 / 4 / 0)$ & $(2 / 3 / 0)$ \\
\hline
\end{tabular}

Tabela 4.38: Total de vitórias, empates e derrotas, em relação às diferenças estatísticas para a avaliação - estimação do viés na classificação para a base de dados Sintética.

mente.

Em resumo, considerando os resultados relativos às diferenças estatísticas identificadas pelo teste de Nemenyi para a predição $\left(\overline{E Q_{N}}\right)$ e para o viés na classificação, os algoritmos KNNImpute, SKNN, IKNNImpute, KMI e EACImpute apresentaram resultados superiores ao algoritmo Média Majority. Não foram verificadas diferenças de desempenho mais contundentes entre os demais algoritmos.

\subsubsection{Base de dados "Íris"}

$\mathrm{Na}$ base de dados Íris os resultados experimentais foram obtidos de 3 formas, conforme procedimento descrito na Seção 4.2. A primeira forma consiste na simulação de valores no atributo $L P$, com probabilidade $p(L P=? \mid C P \in[3,7])=p_{i}$, sendo $p_{i} \in\{0.1,0.3,0.5,0.7\}$ e $i=\{1, \ldots, 4\}$. Para esta primeira forma, são apresentados na Figura 4.23 os resultados referentes à predição com a medida $\overline{E Q_{N}}$. Para efeitos de simplificação, esta primeira forma será chamada de Íris-1. A Figura 4.23 mostra que os algoritmos KNNImpute, SKNN e IKNNImpute apresentaram melhores resultados do que os algoritmos EACImpute, KMI e Média Majority.

Os "ranks" médios obtidos para se realizar o teste de Friedman são apresentados na Tabela 4.39. Nesta tabela, o algoritmo SKNN se destacou com menores valores de "rank" médio. Resultados referentes aos totais de vitórias, empates e derrotas das comparações pareadas dos algoritmos são apresentados na Tabela 4.40, na qual se observa que os algoritmos KNNImpute e SKNN apresentaram os melhores resultados para as taxas de $10 \%$ e $30 \%$ de valores ausentes. Nas demais taxas de valores ausentes o algoritmo SKNN apresentou melhores resultados. Resultados adicionais referentes ao número de vitórias, empates e derrotas dos algoritmos podem ser encontrados na Tabela A.25 do Apêndice.

Diferenças estatísticas entre pares de algoritmos são apresentadas na Tabela A.26 do Apêndice. Na Tabela 4.41 são apresentados os resultados dos totais de vitórias, empates e 


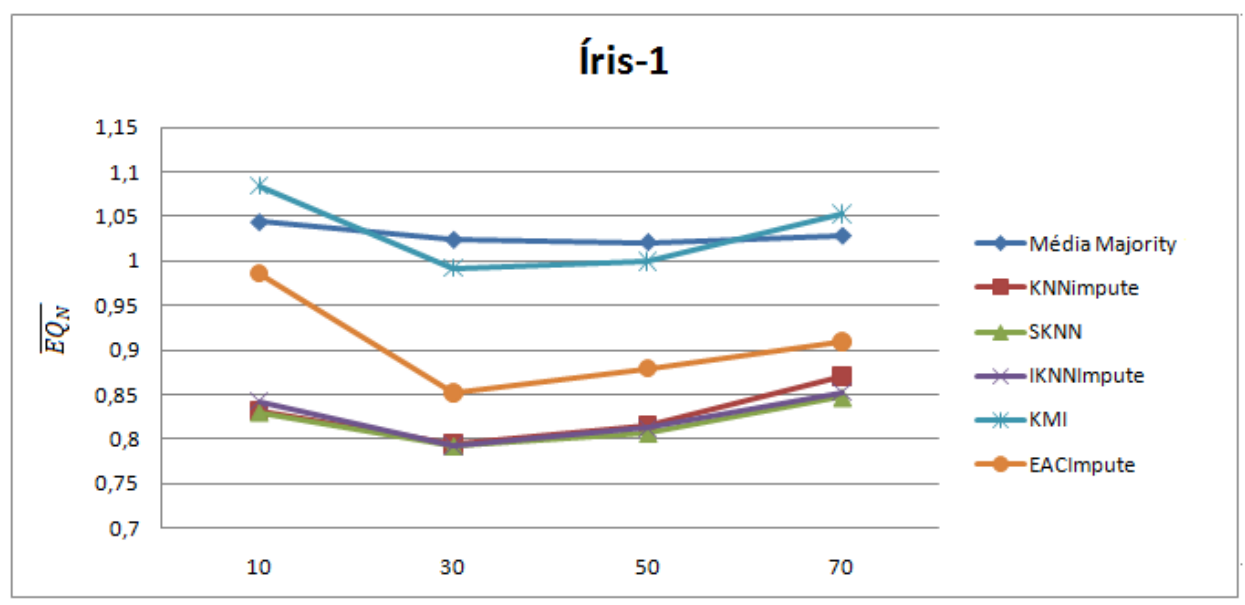

Figura 4.23: Resultados para $\overline{E Q_{N}}$, base de dados Íris-1, para cada cenário de ausência simulados com probabilidades $p(L P=? \mid C P \in[3,7])=p_{i}$, sendo $p_{i} \in\{0.1,0.3,0.5,0.7\}$.

\begin{tabular}{|c|c|c|c|c|c|c|}
\hline $\begin{array}{c}\text { Taxa de } \\
\text { Ausentes }\end{array}$ & Média Majority & KNNImpute & SKNN & IKNNImpute & KMI & EACImpute \\
\hline $10 \%$ & 4,70 & $\mathbf{2 , 5 3}$ & $\mathbf{2 , 5 3}$ & 2,67 & 4,63 & 3,93 \\
\hline $30 \%$ & 4,70 & $\mathbf{2 , 5 3}$ & $\mathbf{2 , 5 3}$ & 2,67 & 4,63 & 3,93 \\
\hline $50 \%$ & 5,53 & 2,53 & $\mathbf{1 , 7 7}$ & 2,23 & 5,37 & 3,57 \\
\hline $70 \%$ & 5,37 & 2,90 & $\mathbf{1 , 8 7}$ & 2,10 & 5,40 & 3,37 \\
\hline $\begin{array}{c}\text { Frequência } \\
\text { dos menores } \\
\text { "ranks" }\end{array}$ & 0 & 2 & 4 & 0 & 0 & 0 \\
\hline
\end{tabular}

Tabela 4.39: "Ranks" médios em cada taxa de ausentes $-\overline{E Q_{N}}$ para a base de dados Íris-1. Os menores valores de "rank" médios estão destacados em negrito.

derrotas de cada algoritmo na comparação pareada. Os algoritmos KNNImpute, SKNN e IKNNImpute apresentaram os melhores resultados nas taxas de $10 \%$ e $30 \%$ de valores ausentes, enquanto que nas demais taxas de valores ausentes o algoritmo SKNN apresentou melhores resultados.

No tocante ao viés de classificação, a Figura 4.24 apresenta os valores médios para cada algoritmo nas taxas de 10\%, 30\%, 50\% e 70\% de valores ausentes. Para todas as taxas de valores ausentes os algoritmos avaliados apresentaram comportamentos semelhantes. A Tabela 4.42 reporta os resultados relativos ao "rank" médio de cada algoritmo para os quais não foram observadas diferenças estatísticas em cada taxa de valores ausentes. Nesta tabela, os algoritmos apresentam um grande equilíbrio entre si.

Em resumo, para a base de dados Íris-1, os resultados relativos às diferenças estatísticas

\begin{tabular}{|c|c|c|c|c|}
\hline Algoritmo & $10 \%$ & $30 \%$ & $50 \%$ & $70 \%$ \\
\hline Média Majority & $(0 / 0 / 5)$ & $(0 / 0 / 5)$ & $(0 / 0 / 5)$ & $(1 / 0 / 4)$ \\
\hline KNNImpute & $(4 / 1 / 0)$ & $(4 / 1 / 0)$ & $(3 / 0 / 2)$ & $(3 / 0 / 2)$ \\
\hline SKNN & $(4 / 1 / 0)$ & $(4 / 1 / 0)$ & $(5 / 0 / 0)$ & $(5 / 0 / 0)$ \\
\hline IKNNImpute & $(3 / 0 / 2)$ & $(3 / 0 / 2)$ & $(4 / 0 / 1)$ & $(4 / 0 / 1)$ \\
\hline KMI & $(1 / 0 / 4)$ & $(1 / 0 / 4)$ & $(1 / 0 / 4)$ & $(0 / 0 / 5)$ \\
\hline EACImpute & $(2 / 0 / 3)$ & $(2 / 0 / 3)$ & $(2 / 3 / 0)$ & $(2 / 3 / 0)$ \\
\hline
\end{tabular}

Tabela 4.40: Total de vitórias, empates e derrotas, em relação aos "ranks" médios $-\overline{E Q_{N}}$ para a base de dados Íris-1. 


\begin{tabular}{|c|c|c|c|c|}
\hline Algoritmo & $10 \%$ & $30 \%$ & $50 \%$ & $70 \%$ \\
\hline Média Majority & $(0 / 2 / 3)$ & $(0 / 2 / 3)$ & $(0 / 1 / 4)$ & $(0 / 1 / 4)$ \\
\hline KNNImpute & $(2 / 3 / 0)$ & $(2 / 3 / 0)$ & $(2 / 3 / 0)$ & $(2 / 3 / 0)$ \\
\hline SKNN & $(2 / 3 / 0)$ & $(2 / 3 / 0)$ & $(3 / 2 / 0)$ & $(3 / 2 / 0)$ \\
\hline IKNNImpute & $(2 / 3 / 0)$ & $(2 / 3 / 0)$ & $(2 / 3 / 0)$ & $(2 / 3 / 0)$ \\
\hline KMI & $(0 / 2 / 3)$ & $(0 / 2 / 3)$ & $(0 / 1 / 4)$ & $(0 / 1 / 4)$ \\
\hline EACImpute & $(0 / 5 / 0)$ & $(0 / 5 / 0)$ & $(2 / 2 / 1)$ & $(2 / 2 / 1)$ \\
\hline
\end{tabular}

Tabela 4.41: Total de vitórias, empates e derrotas, em relação às diferenças estatísticas, para cada cenário de ausência $-\overline{E Q_{N}}$ para a base de dados Íris-1.

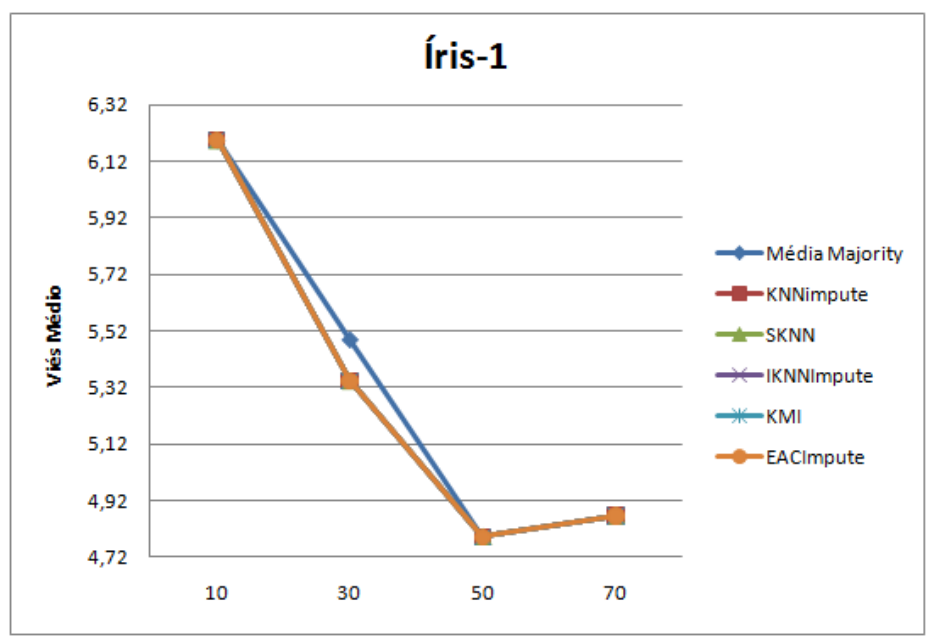

Figura 4.24: Resultados para a estimação do viés de classificação, base de dados Íris-1, para cada taxa de valores ausentes simulados com probabilidade $p(L P=$ ? $\mid 3 \leq C P \leq 7)=p_{i}$, na qual $p_{i} \in\{0.1,0.3,0.5,0.7\}$ e $i=\{1, \ldots, 4\}$. 


\begin{tabular}{|c|c|c|c|c|c|c|}
\hline $\begin{array}{c}\text { Taxa de } \\
\text { Ausentes }\end{array}$ & Média Majority & KNNImpute & SKNN & IKNNImpute & KMI & EACImpute \\
\hline $10 \%$ & $\mathbf{3 , 5 0}$ & $\mathbf{3 , 5 0}$ & $\mathbf{3 , 5 0}$ & $\mathbf{3 , 5 0}$ & $\mathbf{3 , 5 0}$ & $\mathbf{3 , 5 0}$ \\
\hline $30 \%$ & 3,58 & 3,48 & $\mathbf{3 , 4 8}$ & $\mathbf{3 , 4 8}$ & $\mathbf{3 , 4 8}$ & $\mathbf{3 , 4 8}$ \\
\hline $50 \%$ & $\mathbf{3 , 5 0}$ & $\mathbf{3 , 5 0}$ & $\mathbf{3 , 5 0}$ & $\mathbf{3 , 5 0}$ & $\mathbf{3 , 5 0}$ & $\mathbf{3 , 5 0}$ \\
\hline $70 \%$ & $\mathbf{3 , 5 0}$ & $\mathbf{3 , 5 0}$ & $\mathbf{3 , 5 0}$ & $\mathbf{3 , 5 0}$ & $\mathbf{3 , 5 0}$ & $\mathbf{3 , 5 0}$ \\
\hline $\begin{array}{c}\text { Frequência } \\
\text { dos menores } \\
\text { "ranks" }\end{array}$ & 3 & 4 & 4 & 4 & 4 & 4 \\
\hline
\end{tabular}

Tabela 4.42: "Ranks" médios em cada taxa de ausentes - estimação do viés de classificação para a base de dados Íris-1. Os menores valores de "rank" médios estão destacados em negrito.

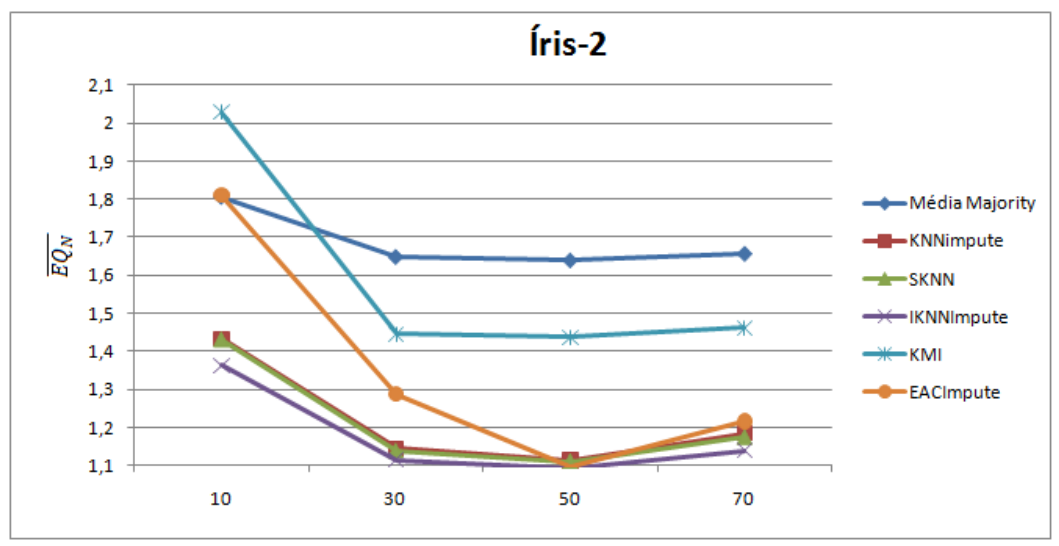

Figura 4.25: Resultados para $\overline{E Q_{N}}$, base de dados Íris-2 para cada taxa de valores ausentes simulados com probabilidades $p(L P=? \mid 4.5 \leq C P \leq 5.1)=p_{i}$, sendo $p_{i} \in\{0.1,0.3,0.5,0.7\}$ e $i=\{1, \ldots, 4\}$.

para o $\overline{E Q_{N}}$ mostram que o algoritmo SKNN apresentou os melhores (ou iguais) resultados do que os demais algoritmos em todas as taxas de valores ausentes. Em relação ao viés de classificação, não foram observadas diferenças estatísticas entre os algoritmos.

Para a segunda forma de simulação de valores no atributo $L P$ da base de dados Íris, com probabilidade $p(L P=? \mid C P \in[4.5,5.1])=p_{i}$, sendo $p_{i} \in\{0.1,0.3,0.5,0.7\}$ e $i=\{1, \ldots, 4\}$, os resultados referentes à predição com $\overline{E Q_{N}}$ são apresentados na Figura 4.25. Para efeitos de simplificação, esta segunda forma será chamada de Íris-2. Nos resultados apresentados na Figura 4.25, os algoritmos KNNImpute, SKNN e IKNNImpute apresentaram comportamentos semelhantes em todas as taxas de valores ausentes, destacando-se com os melhores resultados.

Os valores médios de "ranks" obtidos para se realizar o teste de Friedman são apresentados na Tabela 4.43, na qual o algoritmo IKNNImpute se destacou em todos os cenários de ausência. Os resultados referentes aos totais de vitórias, empates e derrotas das comparações pareadas dos algoritmos são apresentados na Tabela 4.44, na qual se observa que o algoritmo IKNNImpute apresentou melhores resultados para todas as taxas de valores ausentes. Resultados detalhados referentes aos números de vitórias, empates e derrotas dos algoritmos são encontradas na Tabela A.27 do Apêndice.

Procedendo-se com os testes de Friedman e de Nemenyi, foram observadas diferenças estatísticas nas comparações pareadas apresentadas na Tabela A.28 do Apêndice. Na Tabela 4.45 são apresentados os resultados referentes aos totais de vitórias, empates e derrotas de cada algo- 


\begin{tabular}{|c|c|c|c|c|c|c|}
\hline $\begin{array}{c}\text { Taxa de } \\
\text { Ausentes }\end{array}$ & Média Majority & KNNImpute & SKNN & IKNNImpute & KMI & EACImpute \\
\hline $10 \%$ & 5,10 & 2,80 & 2,70 & $\mathbf{2 , 6 7}$ & 4,33 & 3,40 \\
\hline $30 \%$ & 5,53 & 2,93 & 2,57 & $\mathbf{2 , 1 0}$ & 4,10 & 3,77 \\
\hline $50 \%$ & 5,73 & 3,07 & 3,03 & $\mathbf{1 9 7}$ & 4,73 & 2,47 \\
\hline $70 \%$ & 5,73 & 3,07 & 2,87 & $\mathbf{1 , 9 7}$ & 4,23 & 3,13 \\
\hline $\begin{array}{c}\text { Frequência } \\
\text { dos menores } \\
\text { "ranks" }\end{array}$ & 0 & 0 & 0 & 4 & 0 & 0 \\
\hline
\end{tabular}

Tabela 4.43: "Ranks" médios para cada taxa de ausentes $-\overline{E Q_{N}}$ para a base de dados Íris-2. Os menores valores de "rank" médios estão destacados em negrito.

\begin{tabular}{|c|c|c|c|c|}
\hline Algoritmo & $10 \%$ & $30 \%$ & $50 \%$ & $70 \%$ \\
\hline Média Majority & $(0 / 0 / 5)$ & $(0 / 0 / 5)$ & $(0 / 0 / 5)$ & $(0 / 0 / 5)$ \\
\hline KNNImpute & $(3 / 0 / 2)$ & $(3 / 0 / 2)$ & $(2 / 0 / 3)$ & $(3 / 0 / 2)$ \\
\hline SKNN & $(4 / 0 / 1)$ & $(4 / 0 / 1)$ & $(3 / 0 / 2)$ & $(4 / 0 / 1)$ \\
\hline IKNNImpute & $(5 / 0 / 0)$ & $(5 / 0 / 0)$ & $(5 / 0 / 0)$ & $(5 / 0 / 0)$ \\
\hline KMI & $(1 / 0 / 4)$ & $(1 / 0 / 4)$ & $(1 / 0 / 4)$ & $(1 / 0 / 4)$ \\
\hline EACImpute & $(2 / 0 / 3)$ & $(2 / 0 / 3)$ & $(4 / 0 / 1)$ & $(2 / 0 / 3)$ \\
\hline
\end{tabular}

Tabela 4.44: Total de vitórias, empates e derrotas, em relação ao "rank" médio, avaliados por meio do $\overline{E Q_{N}}$ para a base de dados Íris-2.

ritmo na comparação pareada com os demais. Esta tabela sugere um equilíbrio de desempenho entre os algoritmos KNNImpute, SKNN e IKNNImpute, que foram os melhores avaliados segundo este critério.

Para a estimação do viés na classificação, são apresentados na Figura 4.26 os resultados de cada algoritmo nas taxas de 10\%,30\%, 50\% e 70\% de valores ausentes. Nesta figura, todos os algoritmos apresentaram o mesmo comportamento em todas as taxas de valores ausentes. $\mathrm{Na}$ região de simulação de valores ausentes, adotada nessa base de dados, existem algumas instâncias que são cruciais para a definição de fronteiras de decisão (considerando neste caso uma árvore de decisão). Nesse cenário todos os algoritmos obtiveram desempenhos semelhantes, substituindo os valores ausentes por valores que se encontram fora dos limites da fronteira de decisão, influenciando negativamente na classificação. A Tabela 4.46 apresenta os resultados relativos aos "Ranks" médios de cada algoritmo. Nestes resultados não foram observadas diferenças estatísticas para cada taxa de valores ausentes.

Em resumo, considerando os resultados obtidos para o $\overline{E Q_{N}}$ com a aplicação do teste de Nemenyi observa-se que os algoritmos KNNImpute, SKNN e IKNNImpute apresentaram os melhores resultados em todas as taxas de valores ausentes. Em relação à estimação do viés na

\begin{tabular}{|c|c|c|c|c|}
\hline Algoritmo & $10 \%$ & $30 \%$ & $50 \%$ & $70 \%$ \\
\hline Média Majority & $(0 / 1 / 4)$ & $(0 / 0 / 5)$ & $(0 / 1 / 4)$ & $(0 / 0 / 5)$ \\
\hline KNNImpute & $(2 / 3 / 0)$ & $(1 / 4 / 0)$ & $(2 / 3 / 0)$ & $(1 / 4 / 0)$ \\
\hline SKNN & $(2 / 3 / 0)$ & $(2 / 3 / 0)$ & $(2 / 3 / 0)$ & $(1 / 4 / 0)$ \\
\hline IKNNImpute & $(2 / 3 / 0)$ & $(3 / 2 / 0)$ & $(2 / 3 / 0)$ & $(2 / 3 / 0)$ \\
\hline KMI & $(0 / 2 / 3)$ & $(1 / 2 / 2)$ & $(0 / 1 / 4)$ & $(1 / 3 / 1)$ \\
\hline EACImpute & $(1 / 4 / 0)$ & $(1 / 3 / 1)$ & $(2 / 3 / 0)$ & $(1 / 4 / 0)$ \\
\hline
\end{tabular}

Tabela 4.45: Total de vitórias, empates e derrotas, de cada algoritmo que houve diferença estatística na comparação pareada em cada cenário de ausência $-\overline{E Q_{N}}$ para a base de dados Íris-2. 


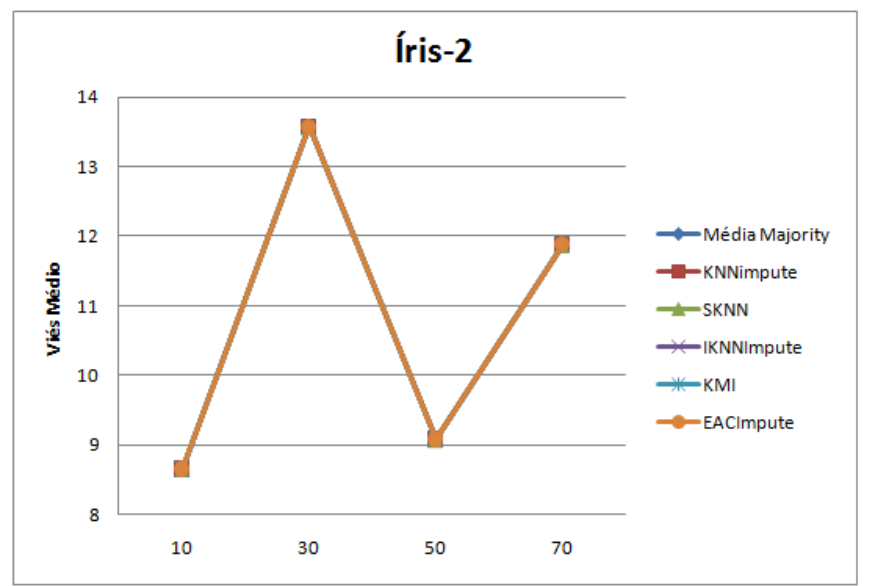

Figura 4.26: Resultados referentes à estimação do viés na classificação, base de dados Íris2, para cada taxa de valores ausentes simulados com probabilidades $p(L P=$ ? $\mid 4.5 \leq C P \leq$ $5.1)=p_{i}$, sendo $p_{i} \in\{0.1,0.3,0.5,0.7\}$.

\begin{tabular}{|c|c|c|c|c|c|c|}
\hline $\begin{array}{c}\text { Taxa de } \\
\text { Ausentes }\end{array}$ & Média Majority & KNNImpute & SKNN & IKNNImpute & KMI & EACImpute \\
\hline $10 \%$ & $\mathbf{3 , 5 0}$ & $\mathbf{3 , 5 0}$ & $\mathbf{3 , 5 0}$ & $\mathbf{3 , 5 0}$ & $\mathbf{3 , 5 0}$ & $\mathbf{3 , 5 0}$ \\
\hline $30 \%$ & $\mathbf{3 , 5 0}$ & $\mathbf{3 , 5 0}$ & $\mathbf{3 , 5 0}$ & $\mathbf{3 , 5 0}$ & $\mathbf{3 , 5 0}$ & $\mathbf{3 , 5 0}$ \\
\hline $50 \%$ & $\mathbf{3 , 5 0}$ & $\mathbf{3 , 5 0}$ & $\mathbf{3 , 5 0}$ & $\mathbf{3 , 5 0}$ & $\mathbf{3 , 5 0}$ & $\mathbf{3 , 5 0}$ \\
\hline $70 \%$ & $\mathbf{3 , 5 0}$ & $\mathbf{3 , 5 0}$ & $\mathbf{3 , 5 0}$ & $\mathbf{3 , 5 0}$ & $\mathbf{3 , 5 0}$ & $\mathbf{3 , 5 0}$ \\
\hline $\begin{array}{c}\text { Frequência } \\
\text { dos menores } \\
\text { "ranks" }\end{array}$ & 4 & 4 & 4 & 4 & 4 & 4 \\
\hline
\end{tabular}

Tabela 4.46: "Ranks" médios em cada taxa de ausentes - estimação do viés na classificação para a base de dados Íris-2. Os menores valores de "rank" médios estão destacados em negrito. 


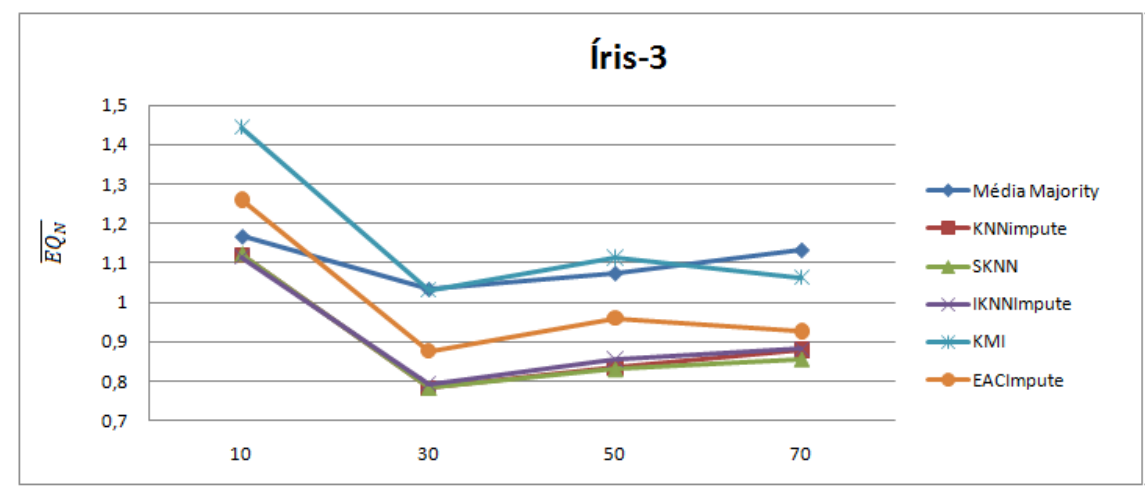

Figura 4.27: Resultados para $\overline{E Q_{N}}$, base de dados Íris-3, para cada cenário de ausência com valores ausentes simulados com probabilidades $p(L P=? \mid 3.5 \leq C P \leq 4.5 \vee 5.1 \leq C P \leq$ $7])=p_{i}$, sendo $p_{i} \in\{0.1,0.3,0.5,0.7\}$.

\begin{tabular}{|c|c|c|c|c|c|c|}
\hline $\begin{array}{c}\text { Taxa de } \\
\text { Ausentes }\end{array}$ & Média Majority & KNNImpute & SKNN & IKNNImpute & KMI & EACImpute \\
\hline $10 \%$ & 4,63 & 2,50 & 2,60 & $\mathbf{2 , 2 3}$ & 5,23 & 3,80 \\
\hline $30 \%$ & 5,47 & 2,20 & $\mathbf{1 , 8 7}$ & 2,43 & 5,30 & 3,73 \\
\hline $50 \%$ & 5,17 & 2,10 & $\mathbf{1 , 7 3}$ & 2,50 & 5,50 & 4,00 \\
\hline $70 \%$ & 5,73 & 2,73 & $\mathbf{1 , 6 7}$ & 2,93 & 5,03 & 2,90 \\
\hline $\begin{array}{c}\text { Frequência } \\
\text { dos menores } \\
\text { "ranks" }\end{array}$ & 0 & 0 & 3 & 1 & 0 & 0 \\
\hline
\end{tabular}

Tabela 4.47: "Ranks" médios em cada taxa de ausentes $-\overline{E Q_{N}}$ para a base de dados Íris-3. Os menores valores de "rank" médios estão destacados em negrito.

classificação, não foram observadas diferenças estatísticas entre os pares de algoritmos. Em casos de empates de desempenho entre os algoritmos, pode-se sugerir, para esta base de dados, a utilização do algoritmo da Média Majority devido à sua simplicidade e eficiência computacional.

Em relação à terceira forma, que consiste na simulação de valores no atributo $L P$ com probabilidade $p(L P=$ ? $\mid 3.5 \leq C P \leq 4.5 \vee 5.1 \leq C P \leq 7])=p_{i}$, sendo $p_{i} \in\{0.1,0.3,0.5,0.7\}$ e $i=\{1, \ldots, 4\}$, os resultados referentes à predição com $\overline{E Q_{N}}$ são apresentados na Figura 4.27. Seguindo o mesmo padrão de nomenclatura adotado nas bases anteriores, a terceira forma de simulação de valores ausentes será denominada de Íris-3. Nos resultados da Figura 4.27, podese observar que todos os algoritmos apresentam comportamentos semelhantes, com destaque para os algoritmos KNNImpute, SKNN e IKNNImpute.

Os "ranks" médios obtidos para se realizar o teste de Friedman são apresentados na Tabela 4.47. Nesta tabela, o algoritmo SKNN se destacou com os melhores resultados na maioria dos cenários de ausência. A Tabela 4.48 apresenta os resultados obtidos pelo somatório do número de vitórias, empates e derrotas das comparações pareadas dos algoritmos apresentados na Tabela A.29 do Apêndice. Pode-se observar na Tabela 4.48 que o algoritmo IKNNImpute se sobressaiu em relação ao demais algoritmos na taxa de $10 \%$ de valores ausentes. Nas demais taxas de valores ausentes o algoritmo SKNN apresentou melhores resultados.

Para as análises realizadas com o teste de Nemenyi, diferenças estatísticas foram observadas 


\begin{tabular}{|c|c|c|c|c|}
\hline Algoritmo & $10 \%$ & $30 \%$ & $50 \%$ & $70 \%$ \\
\hline Média Majority & $(1 / 0 / 4)$ & $(0 / 0 / 5)$ & $(1 / 0 / 4)$ & $(0 / 0 / 5)$ \\
\hline KNNImpute & $(4 / 0 / 1)$ & $(4 / 0 / 1)$ & $(4 / 0 / 1)$ & $(4 / 0 / 1)$ \\
\hline SKNN & $(3 / 0 / 2)$ & $(5 / 0 / 0)$ & $(5 / 0 / 0)$ & $(5 / 0 / 0)$ \\
\hline IKNNImpute & $(5 / 0 / 0)$ & $(3 / 0 / 2)$ & $(3 / 0 / 2)$ & $(2 / 0 / 3)$ \\
\hline KMI & $(0 / 0 / 5)$ & $(1 / 0 / 4)$ & $(0 / 0 / 5)$ & $(1 / 0 / 4)$ \\
\hline EACImpute & $(2 / 0 / 3)$ & $(2 / 0 / 3)$ & $(2 / 0 / 3)$ & $(3 / 0 / 2)$ \\
\hline
\end{tabular}

Tabela 4.48: Total de vitórias, empates e derrotas, em relação ao "rank" médio $-\overline{E Q_{N}}$ para a base de dados Íris-3.

\begin{tabular}{|c|c|c|c|c|}
\hline Algoritmo & $10 \%$ & $30 \%$ & $50 \%$ & $70 \%$ \\
\hline Média Majority & $(0 / 2 / 3)$ & $(0 / 1 / 4)$ & $(0 / 2 / 3)$ & $(0 / 1 / 4)$ \\
\hline KNNImpute & $(2 / 3 / 0)$ & $(3 / 2 / 0)$ & $(3 / 2 / 0)$ & $(2 / 3 / 0)$ \\
\hline SKNN & $(2 / 3 / 0)$ & $(3 / 2 / 0)$ & $(3 / 2 / 0)$ & $(2 / 3 / 0)$ \\
\hline IKNNImpute & $(3 / 2 / 0)$ & $(2 / 3 / 0)$ & $(3 / 2 / 0)$ & $(2 / 3 / 0)$ \\
\hline KMI & $(0 / 1 / 4)$ & $(0 / 1 / 4)$ & $(0 / 1 / 4)$ & $(0 / 1 / 4)$ \\
\hline EACImpute & $(1 / 3 / 1)$ & $(2 / 1 / 2)$ & $(1 / 1 / 3)$ & $(2 / 3 / 0)$ \\
\hline
\end{tabular}

Tabela 4.49: Total de vitórias, empates e derrotas, em relação às diferenças estatísticas $-\overline{E Q_{N}}$ para a base de dados Íris-3.

nas comparações pareadas dos algoritmos, conforme apresentadas na Tabela A.30 do Apêndice. A Tabela 4.49 apresenta os resultados referentes aos totais de vitórias, empates e derrotas de cada algoritmo nas comparações pareadas com os demais, na qual pode-se observar que os algoritmos KNNImpute, SKNN e IKNNImpute se destacaram em relação aos demais (na maioria das taxas de valores ausentes) e, concomitantemente, apresentam uma grande equilíbrio entre si.

A Figura 4.28 reporta os resultados do viés na classificação, na qual os algoritmos apresentam comportamentos semelhantes em todas as taxas de valores ausentes. A Tabela 4.50, por sua vez, apresenta os valores médios dos "ranks", para os quais não foram observadas diferenças estatísticas em cada taxa de valores ausentes, sugerindo que todos os algoritmo apresentam desempenhos iguais.

Em resumo, considerando os resultados relativos às diferenças estatísticas identificadas pelo teste de Nemenyi para a predição $\left(\overline{E Q_{N}}\right)$, os algoritmos KNNImpute, SKNN e IKNNImpute apresentaram os melhores resultados na maioria das taxas de valores ausentes, enquanto que para a estimação do viés na classificação não foram observadas diferenças estatísticas nas comparações pareadas dos algoritmos. Neste caso, sugerindo uma relação entre os resultados da predição e da classificação, pois os algoritmos KNNImpute, SKNN e IKNNImpute se

\begin{tabular}{|c|c|c|c|c|c|c|}
\hline $\begin{array}{c}\text { Taxa de } \\
\text { Ausentes }\end{array}$ & Média Majority & KNNImpute & SKNN & IKNNImpute & KMI & EACImpute \\
\hline $10 \%$ & $\mathbf{3 , 5 0}$ & $\mathbf{3 , 5 0}$ & $\mathbf{3 , 5 0}$ & $\mathbf{3 , 5 0}$ & $\mathbf{3 , 5 0}$ & $\mathbf{3 , 5 0}$ \\
\hline $30 \%$ & $\mathbf{3 , 5 0}$ & $\mathbf{3 , 5 0}$ & $\mathbf{3 , 5 0}$ & $\mathbf{3 , 5 0}$ & $\mathbf{3 , 5 0}$ & $\mathbf{3 , 5 0}$ \\
\hline $50 \%$ & 3,53 & $\mathbf{3 , 4 3}$ & 3,53 & 3,53 & 3,50 & 3,53 \\
\hline $70 \%$ & 3,65 & 3,57 & $\mathbf{3 , 4 2}$ & 3,47 & 3,45 & 3,45 \\
\hline $\begin{array}{c}\text { Frequência } \\
\text { dos menores } \\
\text { "ranks" }\end{array}$ & 2 & 3 & 3 & 2 & 2 & 2 \\
\hline
\end{tabular}

Tabela 4.50: "Ranks" médios em cada taxa de ausentes - estimação do viés na classificação para a base de dados Íris-3. Os menores valores de "rank" médios estão destacados em negrito. 


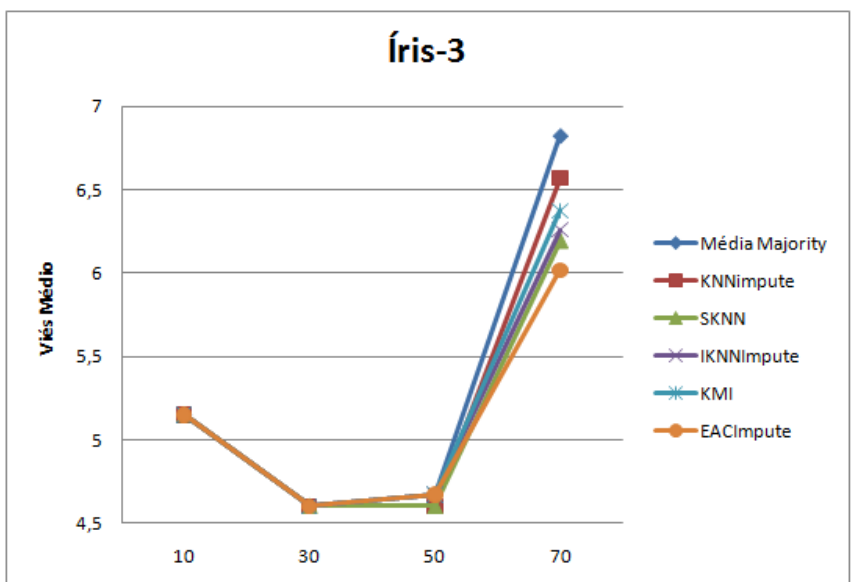

Figura 4.28: Resultados para a estimação do viés de classificação, base de dados Íris-3, para cada taxa de valores ausentes simulados com probabilidades $p(L P=? \mid 3.5 \leq C P \leq 4.5 \vee 5.1 \leq$ $C P \leq 7])=p_{i}$, sendo $p_{i} \in\{0.1,0.3,0.5,0.7\}$.

destacaram em ambas as medidas de avaliação.

\subsubsection{Segmentation}

Nesta base de dados, os resultados obtidos pelos algoritmos de imputação, avaliados pelo $\overline{E Q_{N}}$, são apresentados na Figura 4.29, no qual observa-se que os algoritmos KNNImpute, SKNN, IKNNImpute e EACImpute apresentam mesmo comportamento, se destacando com os melhores resultados. Os valores médios dos "ranks" de cada algoritmo são apresentados na Tabela 4.51, na qual observa-se que o algoritmo SKNN se destacou na maioria dos cenários de ausência. A Tabela 4.52 apresenta os resultados totais relacionados ao número de vitórias, empates e derrotas de cada algoritmo. Nestes resultados, pode-se observar que o algoritmo SKNN apresenta melhores resultados na maioria dos cenários de valores ausentes. Resultados detalhados referentes aos valores do número de vitórias, empates e derrotas dos algoritmos podem ser encontrados na Tabela A.31 do Apêndice.

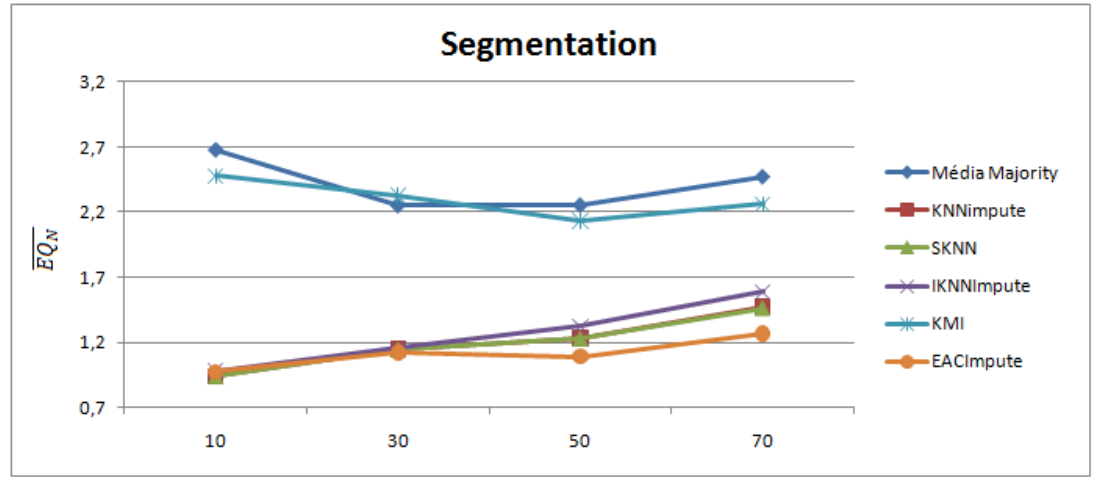

Figura 4.29: Avaliação pelo $\overline{E Q_{N}}$ para a base de dados Segmentation.

Procedendo-se com as análises estatísticas de Friedman e Nemenyi, diferenças estatísticas foram observadas entre pares de algoritmos, conforme apresentadas na Tabela A.32 do 


\begin{tabular}{|c|c|c|c|c|c|c|}
\hline $\begin{array}{c}\text { Taxa de } \\
\text { Ausentes }\end{array}$ & Média Majority & KNNImpute & SKNN & IKNNImpute & KMI & EACImpute \\
\hline $10 \%$ & 5,60 & 2,47 & $\mathbf{2 , 1 7}$ & 2,53 & 5,20 & 3,03 \\
\hline $30 \%$ & 5,30 & 2,23 & $\mathbf{1 , 7 0}$ & 3,40 & 5,53 & 2,83 \\
\hline $50 \%$ & 5,37 & 2,37 & $\mathbf{1 , 9 3}$ & 3,93 & 5,10 & 2,30 \\
\hline $70 \%$ & 5,30 & 2,93 & 2,00 & 3,83 & 4,97 & $\mathbf{1 , 9 7}$ \\
\hline $\begin{array}{c}\text { Frequência } \\
\text { dos menores } \\
\text { "ranks" }\end{array}$ & 0 & 0 & 3 & 0 & 0 & 1 \\
\hline
\end{tabular}

Tabela 4.51: "Ranks" médios em cada taxa de ausentes $-\overline{E Q_{N}}$ para a base de dados Segmentation. Os menores valores de "rank" médios estão destacados em negrito.

\begin{tabular}{|c|c|c|c|c|}
\hline Algoritmo & $10 \%$ & $30 \%$ & $50 \%$ & $70 \%$ \\
\hline Média Majority & $(0 / 0 / 5)$ & $(1 / 0 / 4)$ & $(0 / 0 / 5)$ & $(0 / 0 / 5)$ \\
\hline KNNImpute & $(4 / 0 / 1)$ & $(4 / 0 / 1)$ & $(3 / 0 / 2)$ & $(3 / 0 / 2)$ \\
\hline SKNN & $(5 / 0 / 0)$ & $(5 / 0 / 0)$ & $(5 / 0 / 0)$ & $(4 / 0 / 1)$ \\
\hline IKNNImpute & $(3 / 0 / 2)$ & $(2 / 0 / 3)$ & $(2 / 0 / 3)$ & $(2 / 0 / 3)$ \\
\hline KMI & $(1 / 0 / 4)$ & $(0 / 0 / 5)$ & $(1 / 0 / 4)$ & $(1 / 0 / 4)$ \\
\hline EACImpute & $(2 / 0 / 3)$ & $(3 / 0 / 2)$ & $(4 / 0 / 1)$ & $(5 / 0 / 0)$ \\
\hline
\end{tabular}

Tabela 4.52: Total de vitórias, empates e derrotas, em relação ao "rank" médio $-\overline{E Q_{N}}$ para a base de dados Segmentation.

Apêndice. A Tabela 4.53 apresenta os resultados referentes aos totais de vitórias, empates e derrotas de cada algoritmo, na qual observa-se que os algoritmos KNNImpute, SKNN, IKNNImpute e EACImpute se destacam em relação aos demais algoritmos e, concomitantemente, apresentam um equilíbrio entre si.

A Figura 4.30 apresenta os resultados os resultados médios sob o ponto de vista da estimação do viés na classificação, no qual pode-se observar que os algoritmos KNNImpute, SKNN, IKNNImpute e EACImpute se destacam com os melhores resultados e, simultaneamente, apresentam comportamentos semelhantes. A Tabela 4.54 reporta os valores médios de "rank" de cada algoritmo. Em relação ao número total de vitórias empates e derrotas, na Tabela 4.55 é possível notar que os algoritmos SKNN, IKNNImpute e o EACImpute apresentaram resultados parecidos em relação ao número de vitórias.

Diferenças estatísticas encontradas entre pares de algoritmos são apresentadas na Tabela A.33 do Apêndice. Na Tabela 4.56 são apresentados os resultados referentes aos totais de vitórias, empates e derrotas de cada algoritmo, na qual nota-se que os algoritmos KNNImpute, SKNN, e EACImpute apresentam resultados superiores (ou iguais) em relação aos demais algoritmos em todas as taxas de valores ausentes.

Em resumo, considerando as análises estatísticas realizadas para a capacidade de predição

\begin{tabular}{|c|c|c|c|c|}
\hline Algoritmo & $10 \%$ & $30 \%$ & $50 \%$ & $70 \%$ \\
\hline Média Majority & $(0 / 1 / 4)$ & $(0 / 1 / 4)$ & $(0 / 1 / 4)$ & $(0 / 1 / 4)$ \\
\hline KNNImpute & $(2 / 3 / 0)$ & $(2 / 3 / 0)$ & $(3 / 2 / 0)$ & $(2 / 3 / 0)$ \\
\hline SKNN & $(2 / 3 / 0)$ & $(3 / 2 / 0)$ & $(3 / 2 / 0)$ & $(3 / 2 / 0)$ \\
\hline IKNNImpute & $(2 / 3 / 0)$ & $(2 / 2 / 1)$ & $(1 / 1 / 3)$ & $(1 / 2 / 2)$ \\
\hline KMI & $(0 / 1 / 4)$ & $(0 / 1 / 4)$ & $(0 / 2 / 3)$ & $(0 / 2 / 3)$ \\
\hline EACImpute & $(2 / 3 / 0)$ & $(2 / 3 / 0)$ & $(3 / 2 / 0)$ & $(3 / 2 / 0)$ \\
\hline
\end{tabular}

Tabela 4.53: Total de vitórias, empates e derrotas, em relação às diferenças estatísticas $-\overline{E Q_{N}}$ para a base de dados Segmentation. 


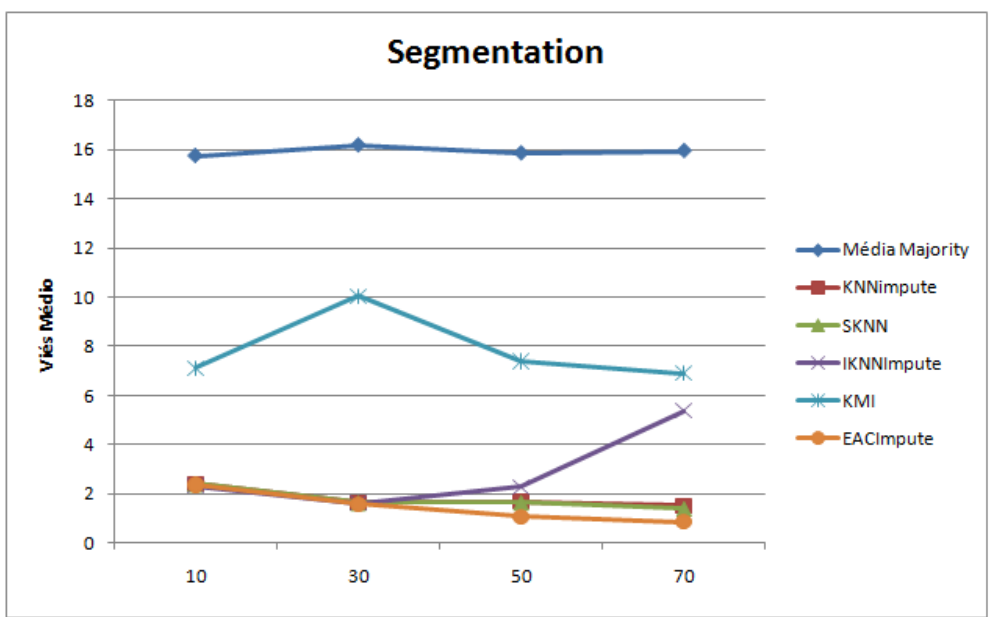

Figura 4.30: Avaliação para o viés de classificação para a base de dados Segmentation.

\begin{tabular}{|c|c|c|c|c|c|c|}
\hline $\begin{array}{c}\text { Taxa de } \\
\text { Ausentes }\end{array}$ & Média Majority & KNNImpute & SKNN & IKNNImpute & KMI & EACImpute \\
\hline $10 \%$ & 5,75 & $\mathbf{2 , 8 0}$ & $\mathbf{2 , 8 0}$ & $\mathbf{2 , 8 0}$ & 4,02 & 2,83 \\
\hline $30 \%$ & 5,88 & 2,68 & 2,68 & 2,55 & 4,62 & $\mathbf{2 , 5 8}$ \\
\hline $50 \%$ & 5,80 & 2,72 & 2,72 & 3,08 & 4,30 & $\mathbf{2 , 3 8}$ \\
\hline $70 \%$ & 5,80 & 2,52 & $\mathbf{2 , 3 2}$ & 4,27 & 3,73 & 2,37 \\
\hline $\begin{array}{c}\text { Frequência } \\
\text { dos menores } \\
\text { "ranks" }\end{array}$ & 0 & 1 & 2 & 1 & 0 & 2 \\
\hline
\end{tabular}

Tabela 4.54: "Ranks" médios em cada taxa de ausentes - estimação do viés de classificação para a base de dados Segmentation. Os menores valores de "rank" médios estão destacados em negrito.

\begin{tabular}{|c|c|c|c|c|}
\hline Algoritmo & $10 \%$ & $30 \%$ & $50 \%$ & $70 \%$ \\
\hline Média Majority & $(0 / 0 / 5)$ & $(0 / 0 / 5)$ & $(0 / 0 / 5)$ & $(0 / 0 / 5)$ \\
\hline KNNImpute & $(3 / 2 / 0)$ & $(2 / 1 / 2)$ & $(3 / 1 / 1)$ & $(3 / 0 / 2)$ \\
\hline SKNN & $(3 / 2 / 0)$ & $(2 / 1 / 2)$ & $(3 / 1 / 1)$ & $(5 / 0 / 0)$ \\
\hline IKNNImpute & $(3 / 2 / 0)$ & $(5 / 0 / 0)$ & $(2 / 0 / 3)$ & $(1 / 0 / 4)$ \\
\hline KMI & $(1 / 0 / 4)$ & $(1 / 0 / 4)$ & $(1 / 0 / 4)$ & $(2 / 0 / 3)$ \\
\hline EACImpute & $(2 / 0 / 3)$ & $(4 / 0 / 1)$ & $(5 / 0 / 0)$ & $(4 / 0 / 1)$ \\
\hline
\end{tabular}

Tabela 4.55: Total de vitórias, empates e derrotas, em relação ao "rank" médio - viés de classificação para a base de dados Segmentation. 


\begin{tabular}{|c|c|c|c|c|}
\hline Algoritmo & $10 \%$ & $30 \%$ & $50 \%$ & $70 \%$ \\
\hline Média Majority & $(0 / 0 / 5)$ & $(0 / 0 / 4)$ & $(0 / 0 / 5)$ & $(0 / 0 / 5)$ \\
\hline KNNImpute & $(1 / 0 / 0)$ & $(2 / 0 / 0)$ & $(2 / 0 / 0)$ & $(2 / 0 / 0)$ \\
\hline SKNN & $(1 / 0 / 0)$ & $(2 / 0 / 0)$ & $(2 / 0 / 0)$ & $(2 / 0 / 0)$ \\
\hline IKNNImpute & $(1 / 0 / 0)$ & $(2 / 0 / 0)$ & $(1 / 0 / 0)$ & $(1 / 0 / 3)$ \\
\hline KMI & $(1 / 0 / 0)$ & $(0 / 0 / 4)$ & $(1 / 0 / 3)$ & $(1 / 0 / 0)$ \\
\hline EACImpute & $(1 / 0 / 0)$ & $(2 / 0 / 0)$ & $(2 / 0 / 0)$ & $(2 / 0 / 0)$ \\
\hline
\end{tabular}

Tabela 4.56: Total de vitórias, empates e derrotas, em relação às diferenças estatísticas - viés de classificação para a base de dados Segmentation.

$\left(\overline{E Q_{N}}\right)$ e viés de classificação com o teste de Nemenyi, os algoritmos KNNImpute, SKNN e o EACImpute obtiveram os melhores resultados na maioria dos cenários de valores ausentes. Neste caso, pode-se afirmar que possivelmente existe correlação entre os resultados de predição e de inserção de viés, pois esses algoritmos se destacaram em ambas avaliações.

\subsubsection{Considerações Finais}

Esta seção sumariza os principais resultados experimentais obtidos nessa dissertação. Nas Seções 4.5.3.1 e 4.5.3.2 são apresentados os resultados obtidos em cada base de dados para o mecanismo MCAR e MAR respectivamente. Além desses resultados, o cálculo do coeficiente de correlação de Pearson entre as duas medidas avaliadas $\left(\overline{E Q_{N}}\right.$ e viés da classificação) para cada base de dados é também apresentado.

\subsubsection{Completamente Aleatório - MCAR}

A Tabela 4.57 sumariza os resultados estatísticos para a base de dados Íris. Para cada taxa de valores ausentes são apresentados os algoritmos que obtiveram melhores resultados (maior número de vitórias) para $\overline{E Q_{N}}$ e para viés de classificação. Considerando os resultados para $\overline{E Q_{N}}$, o algoritmo IKNNImpute apresentou melhores resultados em todas as taxas de valores ausentes, empatando apenas com o algoritmo SKNN nas taxas de $30 \%$ e $50 \%$ de valores ausentes. Para os resultados do viés de classificação, note que os algoritmos IKNNImpute e SKNN fazem parte do conjunto de algoritmos que obtiveram os melhores resultados. Neste caso, é possível concluir que possivelmente existe correlação entre as duas medidas (predição e viés de classificação). Porém, ao considerar os algoritmos que se destacaram em cada cenário de ausência (considerando somente o menor "rank"), o algoritmo IKNNImpute venceu em todos os cenários de ausência para o $\overline{E Q_{N}}$. Ao considerar o viés de classificação, o algoritmo Média Majority obteve melhores resultados, seguido dos algoritmos KNNImpute e KMI. Dessa maneira, pode-se concluir que melhores resultados na predição não implicam necessariamente em melhores resultados na classificação. 


\begin{tabular}{|c|c|c|}
\hline $\begin{array}{c}\text { Taxa de } \\
\text { Ausentes }\end{array}$ & $\overline{E Q_{N}}$ & Viés de classificação \\
\hline \multirow{3}{*}{$10 \%$} & & Média Majority \\
& & KNNImpute \\
& & SKNN \\
& & IKNNImpute \\
& & KMI \\
& & EACImpute \\
\hline \multirow{3}{*}{$30 \%$} & SKNN & Média Majority \\
& IKNNImpute & SNImpute \\
& & IKNNImpute \\
& & KMI \\
& & EACImpute \\
\hline \multirow{2}{*}{$50 \%$} & SKNN & Média Majority \\
& IKNNImpute & KNImpute \\
& & SKNN \\
& & IKNImpute \\
& & EACImpute \\
\hline \multirow{2}{*}{$70 \%$} & IKNNImpute & Média Majority \\
& & EACImpute \\
\hline
\end{tabular}

Tabela 4.57: Resultados gerais para a base de dados Íris com valores ausentes simulados pelo mecanismo MCAR.

Para a base de dados Glass Identification, a Tabela 4.58 apresenta os algoritmos que se destacaram no $\overline{E Q_{N}}$ e no viés de classificação. Nesta tabela, verifica-se que para as taxas de $10 \%$ e $30 \%$ de valores ausentes os algoritmos que se destacaram no $\overline{E Q_{N}}$ não necessariamente se destacaram no viés de classificação. O mesmo se conclui ao se considerar apenas os melhores "ranks" em cada cenário de ausência. Mais especificamente, o algoritmo EACImpute apresentou menores valores de "rank", na maioria dos cenários de ausência para o $\overline{E Q_{N}}$ e não se destacou no resultados do viés de classificação.

\begin{tabular}{|c|c|c|}
\hline $\begin{array}{c}\text { Taxa de } \\
\text { Ausentes }\end{array}$ & $\overline{E Q_{N}}$ & Viés de classificação \\
\hline $10 \%$ & Média Geral & IKNNImpute \\
\hline $30 \%$ & $\begin{array}{c}\text { KNNImpute } \\
\text { IKNNImpute } \\
\text { EACImpute }\end{array}$ & IKNNImpute \\
\hline $50 \%$ & IKNNImpute & IKNNImpute \\
\hline $70 \%$ & IKNNImpute & IKNNImpute \\
\hline
\end{tabular}

Tabela 4.58: Resultados gerais para a base de dados Glass Identification com valores ausentes simulados pelo mecanismo MCAR.

A Tabela 4.59 sumariza os resultados estatísticos obtidos para a base de dados Yeast. Considerando os resultados do $\overline{E Q_{N}}$, os algoritmos que se destacaram nessa medida não obtiveram os melhores resultados no viés de classificação. Esta situação se repete ao considerar os melhores "ranks" para cada cenário de ausência, novamente sugerindo que melhores resultados na predição não implicam necessariamente em melhores resultados na classificação. 


\begin{tabular}{|c|c|c|}
\hline $\begin{array}{c}\text { Taxa de } \\
\text { Ausentes }\end{array}$ & $\overline{E Q_{N}}$ & Viés de classificação \\
\hline $10 \%$ & IKNNImpute & $\begin{array}{c}\text { Média Majority } \\
\text { KMI }\end{array}$ \\
\hline $30 \%$ & $\begin{array}{c}\text { Média Majority } \\
\text { SKNN } \\
\text { IKNNImpute }\end{array}$ & KNNImpute \\
\hline $50 \%$ & $\begin{array}{c}\text { Média Majority } \\
\text { SKNN } \\
\text { IKNNImpute }\end{array}$ & KNNImpute \\
\hline $70 \%$ & $\begin{array}{c}\text { Média Majority } \\
\text { SKNN } \\
\text { IKNNImpute }\end{array}$ & KNNImpute \\
\hline
\end{tabular}

Tabela 4.59: Resultados gerais para a base de dados Yeast com valores ausentes simulados pelo mecanismo MCAR.

Para a base de dados Pen-digts, a Tabela 4.60 apresenta os algoritmos que se destacaram no $\overline{E Q_{N}}$ e no viés de classificação. Nesta tabela, observa-se também que não há uma correlação entre os resultados da predição e os resultados da classificação, pois o algoritmo IKNNImpute, que se destacou na predição, não foi o melhor na classificação. O mesmo ocorre quando se considera os melhores "ranks" em cada cenário de ausência.

\begin{tabular}{|c|c|c|}
\hline $\begin{array}{c}\text { Taxa de } \\
\text { Ausentes }\end{array}$ & $\overline{E Q_{N}}$ & Viés de classificação \\
\hline $10 \%$ & $\begin{array}{c}\text { SKNN } \\
\text { IKNNImpute }\end{array}$ & $\begin{array}{c}\text { KNNImpute } \\
\text { SKNN }\end{array}$ \\
\hline $30 \%$ & IKNNImpute & KNNImpute \\
\hline $50 \%$ & IKNNImpute & KNNImpute \\
\hline $70 \%$ & IKNNImpute & KNNImpute \\
\hline
\end{tabular}

Tabela 4.60: Resultados gerais para a base de dados Pen-Digits com valores ausentes simulados pelo mecanismo MCAR.

Para a base de dados Segmentation, na Tabela 4.61 observa-se que para as taxas de $10 \%$, $30 \%$ e $50 \%$ de valores ausentes apenas o algoritmo IKNNImpute se destaca nas duas medidas ( $\overline{E Q_{N}}$ e viés na classificação). Para a taxa de $70 \%$ de valores ausentes, o algoritmo KNNImpute apresentou melhores resultados no $\overline{E Q_{N}}$ e não obteve melhores resultados no viés de classificação.

\begin{tabular}{|c|c|c|}
\hline $\begin{array}{c}\text { Taxa de } \\
\text { Ausentes }\end{array}$ & $\overline{E Q_{N}}$ & Viés de classificação \\
\hline $10 \%$ & $\begin{array}{c}\text { KNNImpute } \\
\text { SKNN } \\
\text { IKNNImpute }\end{array}$ & IKNNImpute \\
\hline $30 \%$ & $\begin{array}{c}\text { KNNImpute } \\
\text { SKNN } \\
\text { IKNNImpute }\end{array}$ & IKNNImpute \\
\hline $50 \%$ & $\begin{array}{c}\text { KNNImpute } \\
\text { IKNNImpute }\end{array}$ & IKNNImpute \\
\hline $70 \%$ & KNNImpute & IKNNImpute \\
\hline
\end{tabular}

Tabela 4.61: Resultados gerais para a base de dados Segmentation com valores ausentes simulados pelo mecanismo MCAR.

No gráfico da Figura 4.31 são apresentados os resultados sumarizados para o mecanismo MCAR. Estes resultados foram obtidos por meio da contagem do número de vezes que cada 


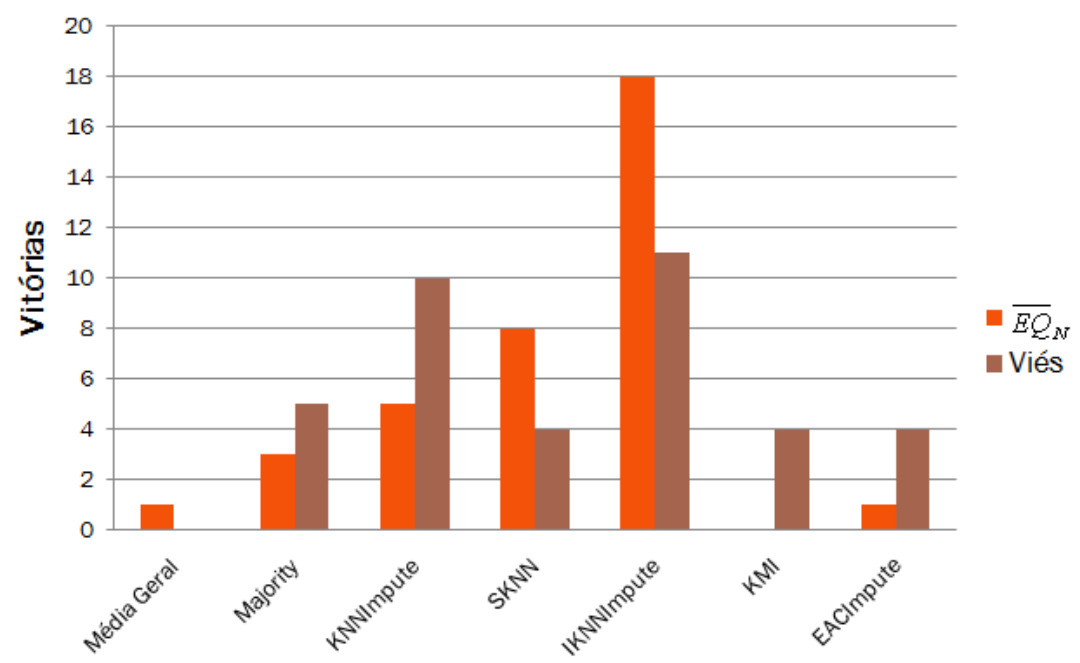

Figura 4.31: Resultados gerais para o mecanismo MCAR.

algoritmo se destacou em cada base de dados. Nesse caso, tem-se cinco bases de dados e quatro taxas de de valores ausentes totalizando vinte casos de possíveis vitórias. Nessa figura, pode-se observar que o algoritmo IKNNImpute obteve melhores resultados na predição. Em relação aos resultados do viés na classificação os algoritmos KNNImpute e IKNNImpute apresentam resultados equilibrados.

Para verificar se há indícios de correlação entre as duas medidas $\left(\overline{E Q_{N}}\right.$ e viés de classificação), na Figura 4.32 é apresentado o gráfico dos resultados do $\overline{E Q_{N}}$ em função dos resultados do viés de classificação. Esses resultados foram obtidos da seguinte maneira. Primeiramente, para cada cenário < taxa de valores ausentes $(10 \%, 30 \%, 50 \%$ e $70 \%)$, quantidade de atributos ausentes $q_{a}$, experimento $e=1 \ldots 30>$ foi identificado o algoritmo que obteve o menor $\overline{E Q_{N}}$. Em seguida, considerando-se todos os cenários foi selecionado o algoritmo que frequentemente obteve menor $\overline{E Q_{N}}$. Para o algoritmo selecionado foram obtidos os resultados de $\overline{E Q_{N}}$ (eixo das abcissas) correspondentes aos cenários nos quais o algoritmo selecionado se destacou e os resultados de viés na classificação (eixo das ordenadas) dos mesmos cenários. Por exemplo, na Figura 4.32(a) o algoritmo IKNNImpute frequentemente obteve menor valor de $\overline{E Q_{N}}$ na maioria dos cenários da base de dados Íris. Além disso, para cada base de dados foi calculado a correlação por meio do bem conhecido coeficiente de correlação de Pearson entre os resultados do $\overline{E Q_{N}}$ e viés na classificação. Pode-se observar que o coeficiente de correlação de Pearson $(\rho)$ para todas as bases de dados se encontra entre $-0,1164$ e 0,2838 sugerindo que não há correlação significativa entre as duas medidas. 


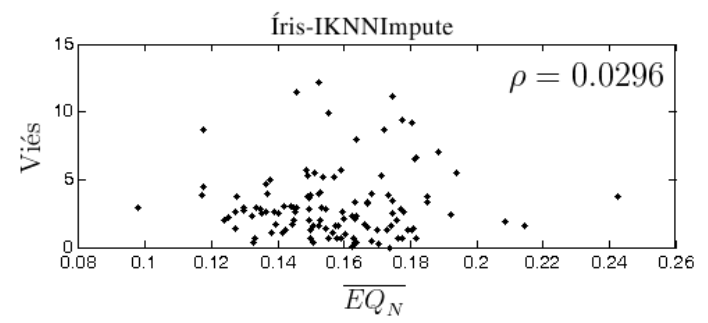

(a) Correlação entre os resultados do $\overline{E Q_{N}}$ e viés de classificação para o algoritmo IKNNImpute na base de dados Íris.

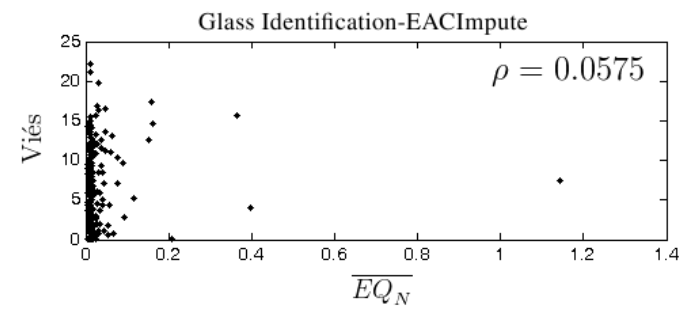

(b) Correlação entre os resultados do $\overline{E Q_{N}}$ e viés de classificação para o algoritmo EACImpute na base de dados Glass Identification.

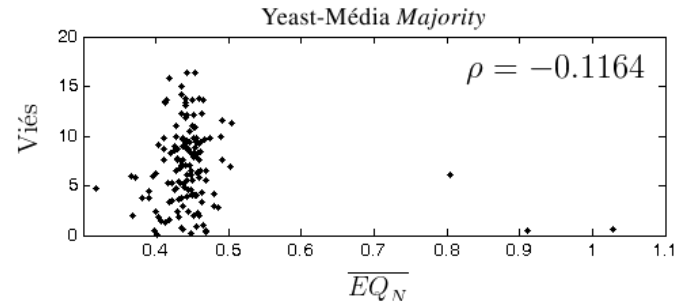

(c) Correlação entre os resultados do $\overline{E Q_{N}}$ e viés de classificação para o algoritmo Média Majority na base de dados Yeast.

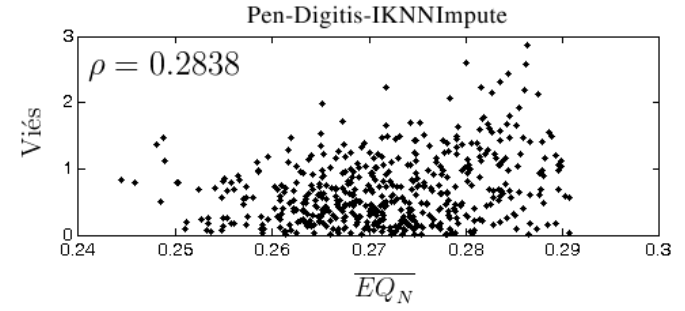

(d) Correlação entre os resultados do $\overline{E Q_{N}}$ e viés de classificação para o algoritmo IKNNImpute na base de dados Pen-Digits.

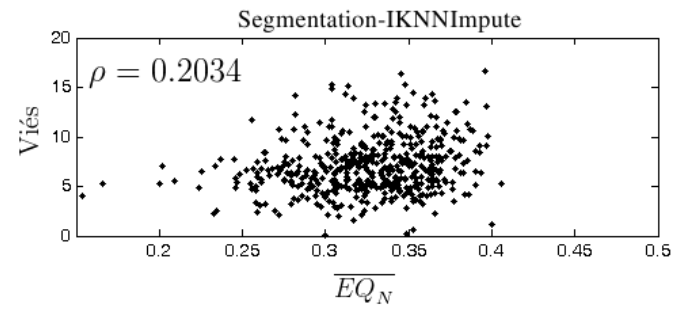

(e) Correlação entre os resultados do $\overline{E Q_{N}}$ e viés de classificação para o algoritmo IKNNImpute na base de dados Segmentation.

Figura 4.32: Correlação entre os resultados do $\overline{E Q_{N}}$ e viés de classificação para o mecanismo MCAR. 


\subsubsection{Aleatório - MAR}

Seguindo as mesmas análises realizas no mecanismo MCAR, a Tabela 4.62 sumariza os resultados estatísticos para a base de dados Sintética. Nesta tabela é possível observar que os algoritmos KMI e EACImpute se destacaram no $\overline{E Q_{N}}$ e no viés de classificação. De maneira análoga ao mecanismo MCAR, a Figura 4.33 apresenta um sumário dos resultados para o mecanismo MAR. Nessa figura pode-se observar que o algoritmo SKNN obteve melhores resultados na predição. Em relação aos resultados do viés na classificação os algoritmos KNNImpute, SKNN e EACImpute apresentaram melhores resultados. Além disso, pode-se observar que o método Majority ocupa o terceiro lugar.

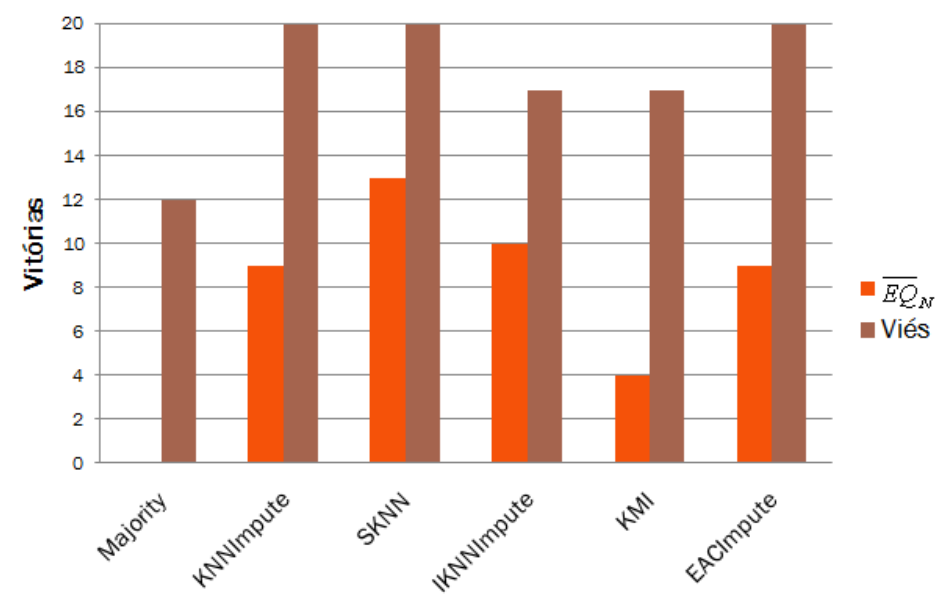

Figura 4.33: Resultados gerais para o mecanismo MAR.

Para verificar se há correlação significativa entre os resultados de predição e classificação, na Figura 4.34 são apresentados os resultados do $\overline{E Q_{N}}$ em função dos resultados do viés de classificação para cada base de dados. Pode-se observar que o coeficiente de correlação de Pearson $(\rho)$ se encontra entre $-0,2450$ e 0,3045 , considerando todas as bases de dados, sugerindo novamente que não há correlação significativa entre as duas medidas $\left(\overline{E Q_{N}}\right.$ e viés de classificação). 


\begin{tabular}{|c|c|c|}
\hline $\begin{array}{c}\text { Taxa de } \\
\text { Ausentes }\end{array}$ & $\overline{E Q_{N}}$ & Viés de classificação \\
\hline & & KNNImpute \\
& SKNN \\
$10 \%$ & KMI & IKNNImpute \\
& EACImpute & KMI \\
& & EACImpute \\
\hline & & KNNImpute \\
& KKMI & IKNNImpute \\
$30 \%$ & EACImpute & KMI \\
& & EACImpute \\
& & KNNImpute \\
& & SKNN \\
$50 \%$ & KMI & IKNNImpute \\
& EACImpute & KMI \\
& & EACImpute \\
\hline & & KNNImpute \\
$70 \%$ & KMI & SKNN \\
& EACImpute & KMI \\
& & EACImpute \\
\hline
\end{tabular}

Tabela 4.62: Resultados gerais para a base de dados Sintética com valores ausentes simulados pelo mecanismo MAR.

\begin{tabular}{|c|c|c|}
\hline $\begin{array}{c}\text { Taxa de } \\
\text { Ausentes }\end{array}$ & $\overline{E Q_{N}}$ & Viés de classificação \\
\hline $10 \%$ & $\begin{array}{l}\text { KNNImpute } \\
\text { SKNN } \\
\text { IKNNImpute }\end{array}$ & $\begin{array}{c}\text { Média Majority } \\
\text { KNNImpute } \\
\text { SKNN } \\
\text { IKNNImpute } \\
\text { KMI } \\
\text { EACImpute }\end{array}$ \\
\hline $30 \%$ & $\begin{array}{l}\text { KNNImpute } \\
\text { SKNN } \\
\text { IKNNImpute }\end{array}$ & $\begin{array}{c}\text { Média Majority } \\
\text { KNNImpute } \\
\text { SKNN } \\
\text { IKNNImpute } \\
\text { KMI } \\
\text { EACImpute }\end{array}$ \\
\hline $50 \%$ & SKNN & $\begin{array}{c}\text { Média Majority } \\
\text { KNNImpute } \\
\text { SKNN } \\
\text { IKNNImpute } \\
\text { KMI } \\
\text { EACImpute }\end{array}$ \\
\hline $70 \%$ & SKNN & $\begin{array}{c}\text { Média Majority } \\
\text { KNNImpute } \\
\text { SKNN } \\
\text { IKNNImpute } \\
\text { KMI } \\
\text { EACImpute }\end{array}$ \\
\hline
\end{tabular}

Tabela 4.63: Resultados gerais para a base de dados Íris-1 com valores ausentes simulados pelo mecanismo MAR. 


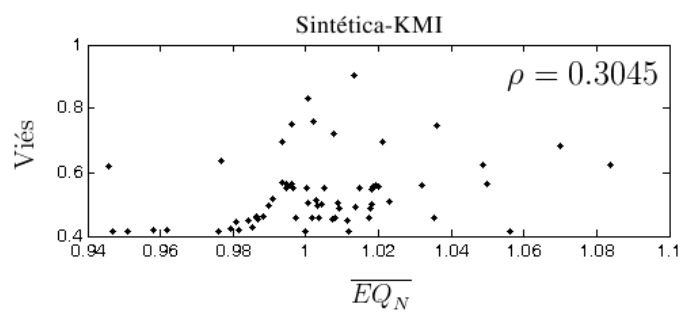

(a) Correlação entre os resultados do $\overline{E Q_{N}}$ e viés de classificação para o algoritmo KMI na base de dados Sintética.

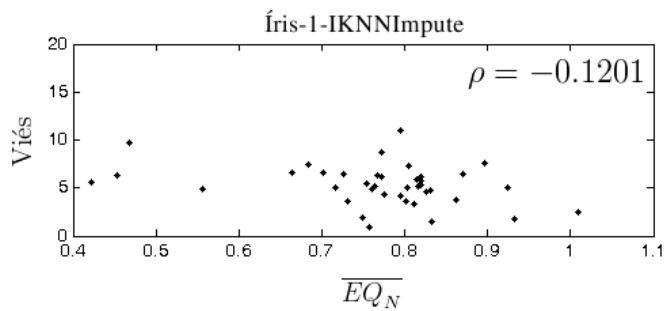

(b) Correlação entre os resultados do $\overline{E Q_{N}}$ e viés de classificação para o algoritmo IKNNImpute na base de dados Íris-1.

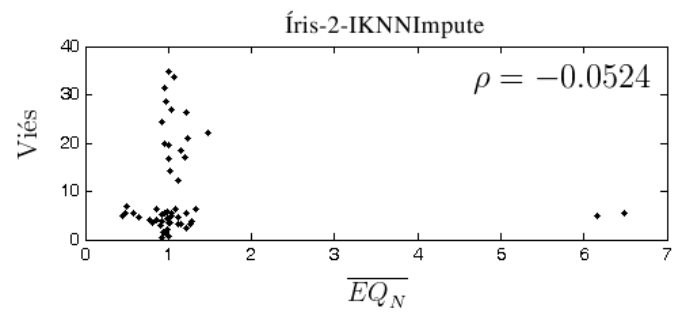

(c) Correlação entre os resultados do $\overline{E Q_{N}}$ e viés de classificação para o algoritmo IKNNImpute na base de dados Íris-2.

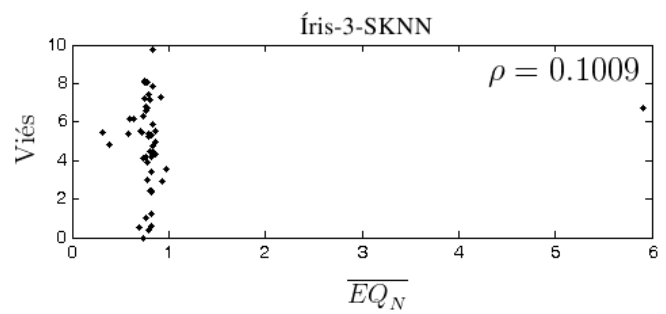

(d) Correlação entre os resultados do $\overline{E Q_{N}}$ e viés de classificação para o algoritmo SKNN na base de dados Íris-3.

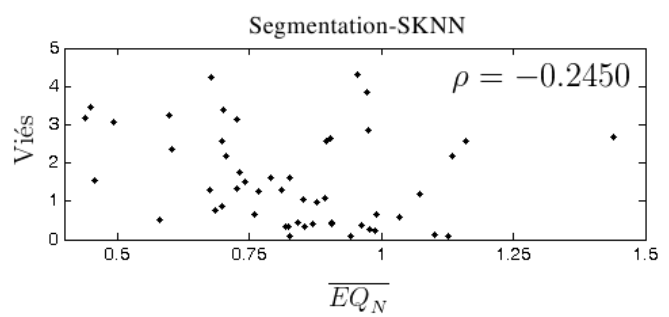

(e) Correlação entre os resultados do $\overline{E Q_{N}}$ e viés de classificação para o algoritmo SKNN na base de dados Segmentation.

Figura 4.34: Correlação entre os resultados do $\overline{E Q_{N}}$ e viés de classificação para o mecanismo MAR. 


\begin{tabular}{|c|c|c|}
\hline $\begin{array}{c}\text { Taxa de } \\
\text { Ausentes }\end{array}$ & $\overline{E Q_{N}}$ & Viés de classificação \\
\hline $10 \%$ & $\begin{array}{l}\text { KNNImpute } \\
\text { SKNN } \\
\text { IKNNImpute }\end{array}$ & $\begin{array}{c}\text { Média Majority } \\
\text { KNNImpute } \\
\text { SKNN } \\
\text { IKNNImpute } \\
\text { KMI } \\
\text { EACImpute }\end{array}$ \\
\hline $30 \%$ & IKNNImpute & $\begin{array}{c}\text { Média Majority } \\
\text { KNNImpute } \\
\text { SKNN } \\
\text { IKNNImpute } \\
\text { KMI } \\
\text { EACImpute }\end{array}$ \\
\hline $50 \%$ & $\begin{array}{l}\text { KNNImpute } \\
\text { SKNN } \\
\text { IKNNImpute } \\
\text { EACImpute }\end{array}$ & $\begin{array}{c}\text { Média Majority } \\
\text { KNNImpute } \\
\text { SKNN } \\
\text { IKNNImpute } \\
\text { KMI } \\
\text { EACImpute }\end{array}$ \\
\hline $70 \%$ & IKNNImpute & $\begin{array}{c}\text { Média Majority } \\
\text { KNNImpute } \\
\text { SKNN } \\
\text { IKNNImpute } \\
\text { KMI } \\
\text { EACImpute }\end{array}$ \\
\hline
\end{tabular}

Tabela 4.64: Resultados gerais para a base de dados Íris-2 com valores ausentes simulados pelo mecanismo MAR.

\begin{tabular}{|c|c|c|}
\hline $\begin{array}{c}\text { Taxa de } \\
\text { Ausentes }\end{array}$ & $\overline{E Q_{N}}$ & Viés de classificação \\
\hline \multirow{5}{*}{$10 \%$} & & Média Majority \\
& & KNNImpute \\
& IKNNImpute & SKNN \\
& & IKNNImpute \\
& & KMI \\
& & EACImpute \\
\hline \multirow{5}{*}{$30 \%$} & KNNImpute & KNNImpute \\
& SKNN & SKNN \\
& & IKNNImpute \\
& & KMI \\
& & EACImpute \\
\hline \multirow{5}{*}{$50 \%$} & KNNImpute & Média Majority \\
& SKNN & SKNN \\
& IKNNImpute & IKNNImpute \\
& & KMI \\
& & EACImpute \\
\hline \multirow{5}{*}{$70 \%$} & KNNImpute & Média Majority \\
& KNNImpute \\
& SKNN & SKNN \\
& EACImpute & IKNNImpute \\
& & KMI \\
& & EACImpute \\
\hline
\end{tabular}

Tabela 4.65: Resultados gerais para a base de dados Íris-3 com valores ausentes simulados pelo mecanismo MAR. 


\begin{tabular}{|c|c|c|}
\hline $\begin{array}{l}\text { Taxa de } \\
\text { Ausentes }\end{array}$ & $\overline{E Q_{N}}$ & Viés de classificação \\
\hline $10 \%$ & $\begin{array}{l}\text { KNNImpute } \\
\text { SKNN } \\
\text { IKNNImpute } \\
\text { EACImpute }\end{array}$ & $\begin{array}{l}\text { KNNImpute } \\
\text { SKNN } \\
\text { IKNNImpute } \\
\text { KMI } \\
\text { EACImpute }\end{array}$ \\
\hline $30 \%$ & SKNN & $\begin{array}{l}\text { KNNImpute } \\
\text { SKNN } \\
\text { IKNNImpute } \\
\text { EACImpute }\end{array}$ \\
\hline $50 \%$ & $\begin{array}{l}\text { KNNImpute } \\
\text { SKNN } \\
\text { EACImpute }\end{array}$ & $\begin{array}{l}\text { KNNImpute } \\
\text { SKNN } \\
\text { EACImpute }\end{array}$ \\
\hline $70 \%$ & $\begin{array}{c}\text { SKNN } \\
\text { EACImpute }\end{array}$ & $\begin{array}{l}\text { KNNImpute } \\
\text { SKNN } \\
\text { EACImpute }\end{array}$ \\
\hline
\end{tabular}

Tabela 4.66: Resultados gerais para a base de dados Segmentation com valores ausentes simulados pelo mecanismo MAR. 


\section{- \\ Conclusão e Trabalhos Futuros}

\subsection{Conclusões}

Diversas aplicações de mineração de dados requerem que os valores ausentes sejam substituídos (imputados). Isto ocorre porque a maioria das técnicas de aprendizagem de máquina necessita que a base de dados esteja com valores conhecidos em todos os atributos. Assim sendo, vários métodos para imputação foram propostos na literatura. Neste trabalho foi proposto um algoritmo evolutivo baseado em agrupamento de dados para imputação, denominado EACImpute. Tradicionalmente, os métodos de imputação são avaliados somente em termos dos resultados da predição, que não permitem estimar a influência dos valores imputados em problemas de classificação. Nesta dissertação, os algoritmos foram avaliados em seis bases de dados, por duas medidas de avaliação: predição e estimação do viés na classificação. Simulações de valores ausentes segundo mecanismos de ausências conhecidos como MCAR e MAR foram realizadas. Resultados experimentais foram obtidos por meio da comparação do EACImpute com seis algoritmos da literatura. Os testes não paramétricos de Friedman e Nemenyi foram aplicados nos resultados obtidos a partir de 77.040 cenários de ausência para verificar se há diferença estatística entre os algoritmos considerados.

Os resultados experimentais para o mecanismo MCAR, na predição, mostraram que o algoritmo IKNNImpute se destacou em 18 dos 20 casos avaliados (considerando os pares $<$ taxa de valores ausentes, base de dados $>$ ). Em relação ao viés de classificação, os algoritmos KNNImpute e IKNNimpute obtiveram melhores resultados, demonstrando um grande equilíbrio entre si. Além disso, para a maioria das bases de dados pode-se concluir que melhores resultados de predição não implicam necessariamente em melhores resultados na classificação.

Em relação aos resultados para o mecanismo MAR, o algoritmo SKNN se destacou na maioria dos casos (aproximadamente 65\%) considerando-se os resultados de predição. Para o viés 
de classificação, os algoritmos KNNImpute, SKNN e EACImpute obtiveram melhores resultados em todas as bases de dados, demonstrando um grande equilíbrio entre si. Além disso, observou-se que os algoritmos que se destacaram na predição fazem parte de um conjunto de algoritmos que se destacaram na classificação. Analisando os resultados de predição e viés de classificação dos algoritmos verificou-se uma fraca correlação entre as medidas, sugerindo que melhores resultados na predição não implicam necessariamente em melhores resultados na classificação. Mesmo que houvesse forte correlação entre as medidas não é possível estimar a influência dos valores imputados em problemas de classificação apenas considerando os resultados de predição. Além disso, foi possível observar como o algoritmo da Média Majority pode afetar significativamente os resultados na classificação por introduzir padrões que não condizem com aqueles induzidos por um classificador.

\subsection{Contribuições}

As contribuições dos estudos realizados nesta dissertação foram:

- Desenvolvimento e avaliação de um método de imputação baseado em algoritmos evolutivos para agrupamento de dados e no conceito dos vizinhos mais próximos. $\mathrm{O}$ algoritmo proposto foi concebido de tal forma a procurar preencher as lacunas relacionadas aos parâmetros críticos de outros algoritmos baseados nesses conceitos.

- Realização de experimentos comparativos entre o EACImpute e seis algoritmos relacionados, em seis bases de dados, com valores ausentes simulados pelos mecanismos MCAR e MAR, incluindo análises estatísticas dos resultados obtidos.

- Verificação da influência do processo de imputação em problemas de classificação. Constatou-se que melhores resultados na predição não implicam necessariamente em melhores resultados na classificação.

- Publicação dos principais resultados referentes ao mecanismo MCAR em anais de conferências internacionais (de Andrade Silva e Hruschka (2009a) e de Andrade Silva e Hruschka (2009b)).

\subsection{Publicações Geradas}

- SILVA, J. A. ; Hruschka, E.R. . An Evolutionary Algorithm for Missing Values Substitution in Classification Tasks. In: The 4th International Conference on Hybrid Artificial Intelligence Systems (HAIS'09), 2009, Salamanca. Lecture Notes in Artificial Intelligence. Berlin : Springer, 2009. v. 5572. p. 195-202. (Publicado) 
Este artigo apresenta os resultados iniciais da comparação do algoritmo proposto, denominado EAC-I, com os algoritmos KNNImpute, SKNN e IKNNImpute em problemas de classificação. Os experimentos foram conduzidos usando o mecanismo MCAR em uma base de dados. Os resultados preliminares obtidos nesse artigo sugerem que melhores resultados de predição não implicam necessariamente em melhores resultados de classificação.

- SILVA, J. A. ; Hruschka, E.R. . EACImpute: An Evolutionary Algorithm for Clustering-Based Imputation. In: 9th International Conference on Intelligent Systems Design and Applications (ISDA'09), 2009, Pisa. Proceedings of The 9th International Conference on Intelligent Systems Design and Applications, 2009. p. 1400-1406. (Publicado)

Este artigo apresenta um estudo aprofundado dos algoritmos de imputação (Método $M a$ jority, KNNImpute, SKNN, IKNNImpute e KMI) em problemas de classificação. Uma modificação do algoritmo proposto anteriormente, agora denominado EACImpute, foi realizada para que o algoritmo possa considerar não somente instâncias com valores conhecidos em todos os seus atributos, mas também considerar instâncias com valores ausentes em alguns atributos durante as imputações. Esta modificação permite que a informação contida nas instâncias com valores ausentes possa ser explorada no processo de imputação. Experimentos foram conduzidos em cinco bases de dados com o mecanismo de simulação MCAR. Os resultados analisados estatisticamente por meio dos testes de Friedman e Nemenyi sugerem que melhores resultados de predição não implicam necessariamente em melhores resultados de classificação.

\subsection{Trabalhos Futuros}

Uma sugestão para trabalhos futuros envolve a definição de novos critérios para determinar, de maneira automática, o número de vizinhos mais próximos para o EACImpute, o que poderia aumentar o desempenho do algoritmo. Na eventualidade de um grupo possuir uma parcela significativa das instâncias do conjunto de dados, essa quantidade de dados pode influenciar negativamente na qualidade da imputação. Isto ocorre porque o algoritmo considera os valores conhecidos de todas as instância de um grupo. Porém, a contribuição de cada valor conhecido é proporcional à distância em relação à uma instância com valores ausentes. Ao contrário dos algoritmos KNNImpute, SKNN e IKNNImpute que provavelmente utilizam um conjunto de vizinhos menor do que o EACImpute, motivo pelo qual pode propiciar melhores resultados na maioria dos cenários avaliados. Outra sugestão envolve analisar a sensibilidade dos parâmetros (número de gerações e o tamanho da população) do EACImpute, bem como a análise de convergência, eficiência computacional e definir novas estratégias para inicializar as partições. Além disso, analisar a escalabilidade dos algoritmos avaliados. 


\section{Referências Bibliográficas}

Acuna, E. e Rodriguez, C. (2004). The treatment of missing values and its effect in the classifier accuracy. Classification, Clustering and Data Mining Applications, páginas 639-648. Citado nas páginas 8 e 14 .

Anderson, R. L. (1946). Missing plot techniques. Biometrics, páginas 41-47. Citado na página 7.

Asuncion, A. e Newman, D. (2007). UCI machine learning repository. C Citado na página 27.

Batista, G. E. A. P. A. (2003). Pré-processamento de Dados em Aprendizado de Máquina Supervisionado. Tese de doutorado, ICMC-USP, São Carlos - SP. Citado nas páginas 7, 8, e 12 .

Batista, G. E. A. P. A. e Monard, M. C. (2003). An Analysis of Four Missing Data Treatment Methods for Supervised Learning. Applied Artificial Intelligence, 17(5):519-533. Citado nas páginas 3 e 14.

Bilmes, J. A. (1997). A gentle tutorial on the em algorithm and its application to parameter estimation for gaussian mixture and hidden markov models. Technical report, University of Berkeley. Citado na página 2.

Brazdil, P. e Bruha, I. (1992). A method of processing unknown attribute values by ID3. Em Proceedings of the Fourth International Conference on Computing and Information, páginas 253-256, Washington, DC, USA. IEEE Computer Society. Citado na página 2.

Breiman, L., Friedman, J., Olshen, R., e Stone, C. (1984). Classification and Regression Trees. Wadsworth and Brooks, Monterey, CA. Citado na página 2.

Brás, L. P. e Menezes, J. C. (2007). Improving cluster-based missing value estimation of dna microarray data. Biomolecular Engineering, 24:273-282. Citado nas páginas 3, 8, 11, 13, 14, 24, 27, 34, 39, e 65. 
Cartwright, M. H., Shepperd, M. J., e Song, Q. (2003). Dealing with missing software project data. Software Metrics, IEEE International Symposium on, páginas 154-165. Citado na página 2 .

Cios, K. J., Pedrycz, W., Swiniarski, R. W., e Kurgan, L. A. (2007). Data Mining: A Knowledge Discovery Approach. Springer-Verlag New York, Inc., Secaucus, NJ, USA. Citado nas páginas 1 e 2 .

Conversano, C. e Cappelli, C. (2002). Missing data incremental imputation through tree-based methods. Em Hardle, W. e Ronz, B., editores, Compstat 2002: 14th Conference on Computational Statistics, páginas 455-460. Citado na página 2.

Davis, L. (1996). Handbook of Genetic Algorithms. International Thomson Computer Press. Citado na página 20.

de Andrade Silva, J. e Hruschka, E. R. (2009a). EACImpute: An evolutionary algorithm for clustering-based imputation. Em Proceedings of the 2009 9th International Conference on Intelligent Systems Design and Applications, páginas 1400-1406, Washington, DC, USA. IEEE Computer Society. Citado nas páginas 24 e 94.

de Andrade Silva, J. e Hruschka, E. R. (2009b). An evolutionary algorithm for missing values substitution in classification tasks. Em Proceedings of the 4th International Conference on Hybrid Artificial Intelligence Systems, páginas 195-202, Berlin, Heidelberg. Springer-Verlag. Citado na página 94.

Delavallade, T. e Dang, T. H. (2007). Using entropy to impute missing data in a classification task. Em IEEE International Conference on Fuzzy Systems, páginas 1-6. Citado na página 34 .

Dempster, A. P., Laird, N. M., e Rubin, D. B. (1977). Maximum likelihood from incomplete data via the EM algorithm. Journal of the Royal Statistical Society. Series B (Methodological), 39:1-38. Citado nas páginas 2 e 7.

Demsar, J. (2006). Statistical comparisons of classifiers over multiple data sets. Journal of Machine Learning Research, 7:1-30. Citado nas páginas 27 e 40.

Eiben, A. E. e Smith, J. E. (2003). Introduction to Evolutionary Computing (Natural Computing Series). Springer. Citado nas páginas 20 e 21.

Everitt, B. S., Landau, S., e Leese, M. (2001). Cluster Analysis. Arnold Publishers. Citado na página 18.

Fayyad, U. M., Piatetsky-Shapiro, G., e Smyth, P. (1996). From data mining to knowledge discovery: an overview. Em Advances in knowledge discovery and data mining. American Association for Artificial Intelligence, Menlo Park, CA, USA. Citado nas páginas 1 e 3. 
Fogel, D. B. (1999). Evolutionary Computation: Towards a New Philosophy of Machine Intelligence. Wiley-IEEE Press. Citado na página 20.

Freitas, A. A. (2002). Data Mining and Knowledge Discovery with Evolutionary Algorithms. Springer-Verlag New York, Inc., Secaucus, NJ, USA. Citado na página 17.

García, S. e Herrera, F. (2008). An extension on "Statistical Comparisons of Classifiers over Multiple Data Sets" for all pairwise comparisons. Journal of Machine Learning Research, 9:2677-2694. Citado na página 40.

Garcia, A. J. T. e Hruschka, E. R. (2005). Naive bayes as an imputation tool for classification problems. Hybrid Intelligent Systems, International Conference on, páginas 497-499. Citado nas páginas 29,34 , e 65 .

Garner, S. (1995). Weka: The waikato environment for knowledge analysis. Citado na página 30.

Ghahramani, Z. e Jordan, M. I. (1994). Learning from incomplete data. Technical report, Massachusetts Institute of Technology, Cambridge, MA, USA. Citado na página 2.

Han, J. e Kamber, M. (2000). Data Mining: Concepts and Techniques (The Morgan Kaufmann Series in Data Management Systems). Morgan Kaufmann. Citado na página 1.

Hollander, M. e Wolfe, D. A. (1999). Nonparametric statistical methods. A Wiley publication in applied statistics. Wiley, New York, 2nd edition. Citado nas páginas 27 e 40.

Hruschka, E., Campello, R., Freitas, A., e de Carvalho, A. (2009a). A survey of evolutionary algorithms for clustering. IEEE Transactions on Systems, Man and Cybernetics, Part C: Applications and Reviews, 39(2):133-155. Citado na página 17.

Hruschka, E. R., Campello, R. J. G. B., e de Castro, L. N. (2006). Evolving clusters in geneexpression data. Information Science, 176(13):1898-1927. Citado nas páginas 4, 15, 17, 18, e 20.

Hruschka, E. R., Garcia, A. J. T., Jr., E. R. H., e Ebecken, N. F. F. (2009b). On the influence of imputation in classification: Practical issues. Journal of Experimental and Theoretical Artificial Intelligence, 21:43-58. Citado nas páginas 4, 34, 35, 36, 37, e 38.

Hruschka, E. R., Junior, E. R. H., e Ebecken, N. F. F. (2004). Towards efficient imputation by nearest-neighbors: A clustering-based approach. Em Proceedings of the 6th Australian Joint Conference on Artificial Intelligence, Lecture Notes in Computer Science, páginas 513-525. Spring-Verlag. Citado nas páginas 3, 12, 13, 14, 24, 27, 39, e 65.

Johansson, P. e Hakkinen, J. (2006). Improving missing value imputation of microarray data by using spot quality weights. BMC Bioinformatics. Citado na página 2. 
Jonsson, P. e Wohlin, C. (2004). An evaluation of k-nearest neighbour imputation using likert data. Em Proceedings of the Software Metrics, 10th International Symposium, páginas 108118, Washington, DC, USA. IEEE Computer Society. Citado nas páginas 3 e 8.

Jordan, M. I. e Xu, L. (1995). Convergence results for the em approach to mixtures of experts architectures. Neural Networks, 8:1409-1431. Citado na página 2.

Kaufman, L. e Rousseeuw, P. J. (1990). Finding Groups in Data - An Introduction to Cluster Analysis. Wiley Series in Probability and Statistics. Wiley-Interscience. Citado nas páginas 14 e 20.

Kerr, M. K., Martin, M., e Churchill, G. A. (2000). Analysis of variance for gene expression microarray data. Journal of Computational Biology, 7:819-837. Citado na página 3.

Kim, D.-W., Lee, K.-Y., Lee, K. H., e Lee, D. (2007). Towards clustering of incomplete microarray data without the use of imputation. Bioinformatics, 23(1):107-113. Citado na página 14.

Kim, H., Golub, G. H., e Park, H. (2005). Missing value estimation for dna microarray gene expression data: local least squares imputation. Bioinformatics, 21(2):187-198. Citado na página 34.

Kim, K.-Y., Kim, B.-J., e Yi, G.-S. (2004). Reuse of imputed data in microarray analysis increases imputation efficiency. BMC Bioinformatics, 5:160. Citado nas páginas 2, 3, 8, 10, $11,14,24,27,39$, e 65.

Kohavi, R. e John, G. H. (1997). Wrappers for feature subset selection. Artificial Intelligence, 97(1-2):273-324. Citado na página 30.

Kononenko, I., Bratko, I., e Roskar, E. (1984). Experiments in automatic learning of medical diagnostic rules. Technical report, Jozef Stefan Institute, Ljubjana, Yogoslavia. Citado nas páginas 8,27 , e 65 .

Lakshminarayan, K., Harp, S. A., Goldman, R. P., e Samad, T. (1996). Imputation of missing data using machine learning techniques. Em Second International Conference on Knowledge Discovery and Data Mining, páginas 140-145. Citado na página 2.

Little, R. J. A. e Rubin, D. B. (2002). Statistical analysis with missing data. John Wiley \& Sons, Inc., New York, NY, USA, second edition. Citado na página 5.

Lobo, O. O. e Numao, M. (2000). Ordered estimation of missing values for propositional learning. Journal of the Japanese Society for Artificial Intelligence, 15(1):162-168. Citado na página 2 . 
Maimon, O. e Rokach, L. (2005). Data Mining and Knowledge Discovery Handbook. Springer. Citado na página 17.

Mitchell, T. M. (1997). Machine Learning. McGraw-Hill, New York. Citado na página 1.

Nguyen, D. V., Wang, N., e Carroll, R. J. (2004). Evaluation of missing value estimation for microarray data. Journal of Data Science, 2:347-370. Citado na página 7.

Oba, S., Sato, M.-A., Takemasa, I., Monden, M., Matsubara, K. I., e Ishii, S. (2003). A bayesian missing value estimation method for gene expression profile data. Bioinformatics, 19(16):2088-2096. Citado na página 34.

Pedrycz, W. (2005). Knowledge-Based Clustering: From Data to Information Granules. WileyInterscience. Citado na página 3.

Pipino, L. L., Lee, Y. W., e Wang, R. Y. (2002). Data quality assessment. Communications of the ACM, 45(4):211-218. Citado na página 1.

Preece, A. D. (1971). Iterative procedures for missing values in experiments. Technometrics, páginas 743-753. Citado na página 7.

Pyle, D. (1999). Data Preparation for Data Mining (The Morgan Kaufmann Series in Data Management Systems). Morgan Kaufmann. Citado nas páginas 2, 36, e 37.

Qin, Y., Zhang, S., Zhu, X., Zhang, J., e Zhang, C. (2009). Pop algorithm: Kernel-based imputation to treat missing values in knowledge discovery from databases. Expert Syst. Appl., 36(2):2794-2804. Citado na página 7.

Quinlan, J. R. (1993). Induction of decision trees. Machine Learning, 1:81-106. Citado na página 2.

Redner, R. A. e Walker, H. F. (1984). Mixture densities, maximum likelihood and the EM algorithm. SIAM Review, 26:195-239. Citado na página 2.

Rubin, D. B. (1977). Formalizing subjective notion about the effects of nonrespondents in samples surveys. Journal of the American Statistical Association, páginas 538-543. Citado nas páginas 2 e 7.

Rubin, D. B. (1987). Multiple imputation for non responses in surveys. New York: WileyInterscience. Citado nas páginas 2 e 7.

Schafer, J. (2000). Analysis of Incomplete Multivariate Data. Chapman \& Hall/CRC. Citado nas páginas 2 e 7.

Schafer, J. e Graham, J. (2002). Missing data: Our view of the state of the art. Psychological Methods. Citado na página 5. 
Scheel, I., Aldrin, M., Glad, I. K., Sørum, R., Lyng, H., e Frigessi, A. (2005). The influence of missing value imputation on detection of differentially expressed genes from microarray data. Bioinformatics, 21(23):4272-4279. Citado na página 3.

Sheng, Q., Moreau, Y., e Moor, B. D. (2003). Biclustering microarray data by gibbs sampling. Bioinformatics, 19:196-205. Citado na página 14.

Tibshirani, R., Hastie, T., Narasimhan, D., e Chu, G. (2002). Diagnosis of multiple cancer types by shrunken centroids of gene expression. Em Proceedings of the National Academy of Sciences, páginas 6567-6572. Citado na página 3.

Troyanskaya, O. G., Cantor, M., Sherlock, G., Brown, P. O., Hastie, T., Tibshirani, R., Botstein, D., e Altman, R. B. (2001). Missing value estimation methods for dna microarrays. Bioinformatics, 17(6):520-525. Citado nas páginas 3, 8, 9, 10, 11, 14, 24, 27, 39, e 65.

Tuikkala, J., Elo, L. L., Nevalainen, O. S., e Aittokallio, T. (2008). Missing value imputation improves clustering and interpretation of gene expression microarray data. BMC Bioinformatics, 9:202+. Citado na página 34 .

Tusher, V., Tibshirani, R., e Chu, G. (2001). Significance analysis of microarrays applied to the ionizing radiation response. Em Proceedings of the National Academy of Sciences, páginas 5116-5121. Citado na página 3.

Wu, X., Kumar, V., Ross Quinlan, J., Ghosh, J., Yang, Q., Motoda, H., McLachlan, G. J., Ng, A., Liu, B., Yu, P. S., Zhou, Z. H., Steinbach, M., Hand, D. J., e Steinberg, D. (2007). Top 10 algorithms in data mining. Knowledge and Information Systems, 14(1):1-37. Citado na página 14.

Zhang, S., Qin, Z., Ling, C. X., e Sheng, S. (2005). "missing is useful': Missing values in costsensitive decision trees. IEEE Transactions on Knowledge and Data Engineering, 17:16891693. Citado na página 7. 


\section{APÊNDICE}

\section{Apêndice}

\section{A.1 Completamente Aleatório - MCAR}

\section{A.1.1 Base de dados "Íris"}

\begin{tabular}{|c|c|c|c|c|c|c|c|c|}
\hline $\begin{array}{c}\text { Taxa de } \\
\text { Ausentes }\end{array}$ & Algoritmos & Média Geral & Média Majority & KNNImpute & SKNN & IKNNImpute & KMI & EACImpute \\
\hline \multirow{7}{*}{$10 \%$} & Média Geral & $(-/-/-)$ & $(0 / 0 / 2)$ & $(0 / 0 / 2)$ & $(0 / 0 / 2)$ & $(0 / 0 / 2)$ & $(0 / 0 / 2)$ & $(0 / 0 / 2)$ \\
\hline & Média Majority & $(2 / 0 / 0)$ & $(-/-/-)$ & $(0 / 0 / 2)$ & $(0 / 0 / 2)$ & $(0 / 0 / 2)$ & $(0 / 0 / 2)$ & $(0 / 0 / 2)$ \\
\hline & KNNImpute & $(2 / 0 / 0)$ & $(2 / 0 / 0)$ & $(-/-/-)$ & $(1 / 0 / 1)$ & $(0 / 0 / 2)$ & $(2 / 0 / 0)$ & $(2 / 0 / 0)$ \\
\hline & SKNN & $(2 / 0 / 0)$ & $(2 / 0 / 0)$ & $(1 / 0 / 1)$ & $(-/-/-)$ & $(0 / 0 / 2)$ & $(2 / 0 / 0)$ & $(2 / 0 / 0)$ \\
\hline & IKNNImpute & $(2 / 0 / 0)$ & $(2 / 0 / 0)$ & $(2 / 0 / 0)$ & $(2 / 0 / 0)$ & $(-/-/-)$ & $(2 / 0 / 0)$ & $(2 / 0 / 0)$ \\
\hline & KMI & $(2 / 0 / 0)$ & $(2 / 0 / 0)$ & $(0 / 0 / 2)$ & $(0 / 0 / 2)$ & $(0 / 0 / 2)$ & $(-/-/-)$ & $(0 / 0 / 2)$ \\
\hline & EACImpute & $(2 / 0 / 0)$ & $(2 / 0 / 0)$ & $(0 / 0 / 2)$ & $(0 / 0 / 2)$ & $(0 / 0 / 2)$ & $(2 / 0 / 0)$ & $(-/-/-)$ \\
\hline \multirow{7}{*}{$30 \%$} & Média Geral & $(-/-/-)$ & $(0 / 0 / 2)$ & $(0 / 0 / 2)$ & $(0 / 0 / 2)$ & $(0 / 0 / 2)$ & $(0 / 0 / 2)$ & $(0 / 0 / 2)$ \\
\hline & Média Majority & $(2 / 0 / 0)$ & $(-/-/-)$ & $(0 / 0 / 2)$ & $(0 / 0 / 2)$ & $(0 / 0 / 2)$ & $(0 / 0 / 2)$ & $(0 / 0 / 2)$ \\
\hline & KNNImpute & $(2 / 0 / 0)$ & $(2 / 0 / 0)$ & $(-/-/-)$ & $(0 / 0 / 2)$ & $(0 / 0 / 2)$ & $(1 / 0 / 1)$ & $(1 / 0 / 1)$ \\
\hline & SKNN & $(2 / 0 / 0)$ & $(2 / 0 / 0)$ & $(2 / 0 / 0)$ & $(-/-/-)$ & $(0 / 0 / 2)$ & $(2 / 0 / 0)$ & $(2 / 0 / 0)$ \\
\hline & IKNNImpute & $(2 / 0 / 0)$ & $(2 / 0 / 0)$ & $(2 / 0 / 0)$ & $(2 / 0 / 0)$ & $(-/-/-)$ & $(2 / 0 / 0)$ & $(2 / 0 / 0)$ \\
\hline & KMI & $(2 / 0 / 0)$ & $(2 / 0 / 0)$ & $(1 / 0 / 1)$ & $(0 / 0 / 2)$ & $(0 / 0 / 2)$ & $(-/-/-)$ & $(0 / 0 / 2)$ \\
\hline & EACImpute & $(2 / 0 / 0)$ & $(2 / 0 / 0)$ & $(1 / 0 / 1)$ & $(0 / 0 / 2)$ & $(0 / 0 / 2)$ & $(2 / 0 / 0)$ & $(-/-/-)$ \\
\hline \multirow{7}{*}{$50 \%$} & Média Geral & $(-/-/-)$ & $(0 / 0 / 2)$ & $(0 / 0 / 2)$ & $(0 / 0 / 2)$ & $(0 / 0 / 2)$ & $(0 / 0 / 2)$ & $(0 / 0 / 2)$ \\
\hline & Média Majority & $(2 / 0 / 0)$ & $(-/-/-)$ & $(0 / 0 / 2)$ & $(0 / 0 / 2)$ & $(0 / 0 / 2)$ & $(0 / 0 / 2)$ & $(0 / 0 / 2)$ \\
\hline & KNNImpute & $(2 / 0 / 0)$ & $(2 / 0 / 0)$ & $(-/-/-)$ & $(0 / 0 / 2)$ & $(0 / 0 / 2)$ & $(1 / 0 / 1)$ & $(1 / 0 / 1)$ \\
\hline & SKNN & $(2 / 0 / 0)$ & $(2 / 0 / 0)$ & $(2 / 0 / 0)$ & $(-/-/-)$ & $(0 / 0 / 2)$ & $(2 / 0 / 0)$ & $(2 / 0 / 0)$ \\
\hline & IKNNImpute & $(2 / 0 / 0)$ & $(2 / 0 / 0)$ & $(2 / 0 / 0)$ & $(2 / 0 / 0)$ & $(-/-/-)$ & $(2 / 0 / 0)$ & $(2 / 0 / 0)$ \\
\hline & KMI & $(2 / 0 / 0)$ & $(2 / 0 / 0)$ & $(1 / 0 / 1)$ & $(0 / 0 / 2)$ & $(0 / 0 / 2)$ & $(-/-/-)$ & $(0 / 0 / 2)$ \\
\hline & EACImpute & $(2 / 0 / 0)$ & $(2 / 0 / 0)$ & $(1 / 0 / 1)$ & $(0 / 0 / 2)$ & $(0 / 0 / 2)$ & $(2 / 0 / 0)$ & $(-/-/-)$ \\
\hline \multirow{7}{*}{$70 \%$} & Média Geral & $(-/-/-)$ & $(0 / 0 / 2)$ & $(0 / 0 / 2)$ & $(0 / 0 / 2)$ & $(0 / 0 / 2)$ & $(0 / 0 / 2)$ & $(0 / 0 / 2)$ \\
\hline & Média Majority & $(2 / 0 / 0)$ & $(-/-/-)$ & $(0 / 0 / 2)$ & $(0 / 0 / 2)$ & $(0 / 0 / 2)$ & $(1 / 0 / 1)$ & $(0 / 0 / 2)$ \\
\hline & KNNImpute & $(2 / 0 / 0)$ & $(2 / 0 / 0)$ & $(-/-/-)$ & $(0 / 0 / 2)$ & $(0 / 0 / 2)$ & $(2 / 0 / 0)$ & $(1 / 0 / 1)$ \\
\hline & SKNN & $(2 / 0 / 0)$ & $(2 / 0 / 0)$ & $(2 / 0 / 0)$ & $(-/-/-)$ & $(0 / 0 / 2)$ & $(2 / 0 / 0)$ & $(2 / 0 / 0)$ \\
\hline & IKNNImpute & $(2 / 0 / 0)$ & $(2 / 0 / 0)$ & $(2 / 0 / 0)$ & $(2 / 0 / 0)$ & $(-/-/-)$ & $(2 / 0 / 0)$ & $(2 / 0 / 0)$ \\
\hline & KMI & $(2 / 0 / 0)$ & $(1 / 0 / 1)$ & $(0 / 0 / 2)$ & $(0 / 0 / 2)$ & $(0 / 0 / 2)$ & $(-/-/-)$ & $(0 / 0 / 2)$ \\
\hline & EACImpute & $(2 / 0 / 0)$ & $(2 / 0 / 0)$ & $(1 / 0 / 1)$ & $(0 / 0 / 2)$ & $(0 / 0 / 2)$ & $(2 / 0 / 0)$ & $(-/-/-)$ \\
\hline
\end{tabular}

Tabela A.1: Resultados da comparação pareada (Vitórias/Empates/derrotas) dos "ranks" médios em cada taxa de ausentes $-\overline{E Q_{N}}$ para base de dados Íris. 


\begin{tabular}{|c|c|c|c|c|}
\hline Pares de Algoritmos & $10 \%$ & $30 \%$ & $50 \%$ & $70 \%$ \\
\hline$<$ Média Geral, Média Majority $>$ & $(0 / 0 / 2)$ & $(0 / 2 / 0)$ & $(0 / 0 / 2)$ & $(0 / 0 / 2)$ \\
\hline$<$ Média Geral, KNNImpute $>$ & $(0 / 0 / 2)$ & $(0 / 0 / 2)$ & $(0 / 0 / 2)$ & $(0 / 0 / 2)$ \\
\hline$<$ Média Geral, SKNN $>$ & $(0 / 0 / 2)$ & $(0 / 0 / 2)$ & $(0 / 0 / 2)$ & $(0 / 0 / 2)$ \\
\hline$<$ Média Geral, IKNNImpute $>$ & $(0 / 0 / 2)$ & $(0 / 0 / 2)$ & $(0 / 0 / 2)$ & $(0 / 0 / 2)$ \\
\hline$<$ Média Geral, KMI $>$ & $(0 / 0 / 2)$ & $(0 / 0 / 2)$ & $(0 / 0 / 2)$ & $(0 / 1 / 1)$ \\
\hline$<$ Média Geral, EACImpute $>$ & $(0 / 0 / 2)$ & $(0 / 0 / 2)$ & $(0 / 0 / 2)$ & $(0 / 0 / 2)$ \\
\hline$<$ Média Majority, KNNImpute $>$ & $(0 / 0 / 2)$ & $(0 / 0 / 2)$ & $(0 / 1 / 1)$ & $(0 / 1 / 1)$ \\
\hline$<$ Média Majority, SKNN $>$ & $(0 / 0 / 2)$ & $(0 / 0 / 2)$ & $(0 / 0 / 2)$ & $(0 / 0 / 2)$ \\
\hline$<$ Média Majority, IKNNImpute $>$ & $(0 / 0 / 2)$ & $(0 / 0 / 2)$ & $(0 / 0 / 2)$ & $(0 / 0 / 2)$ \\
\hline$<$ Média Majority, KMI $>$ & $(0 / 2 / 0)$ & $(0 / 1 / 1)$ & $(0 / 2 / 0)$ & $(0 / 2 / 0)$ \\
\hline$<$ Média Majority, EACImpute $>$ & $(0 / 1 / 1)$ & $(0 / 0 / 2)$ & $(0 / 0 / 2)$ & $(0 / 0 / 2)$ \\
\hline$<$ KNNImpute, SKNN $>$ & $(0 / 2 / 0)$ & $(0 / 1 / 1)$ & $(0 / 0 / 2)$ & $(0 / 1 / 1)$ \\
\hline$<$ KNNImpute, IKNNImpute $>$ & $(0 / 2 / 0)$ & $(0 / 1 / 1)$ & $(0 / 0 / 2)$ & $(0 / 0 / 2)$ \\
\hline$<$ KNNImpute, KMI $>$ & $(1 / 1 / 0)$ & $(1 / 1 / 0)$ & $(1 / 1 / 0)$ & $(1 / 1 / 0)$ \\
\hline$<$ KNNImpute, EACImpute $>$ & $(0 / 2 / 0)$ & $(0 / 2 / 0)$ & $(0 / 1 / 1)$ & $(0 / 2 / 0)$ \\
\hline$<$ SKNN, IKNNImpute $>$ & $(0 / 2 / 0)$ & $(0 / 2 / 0)$ & $(0 / 2 / 0)$ & $(0 / 2 / 0)$ \\
\hline$<$ SKNN, KMI $>$ & $(1 / 1 / 0)$ & $(2 / 0 / 0)$ & $(2 / 0 / 0)$ & $(2 / 0 / 0)$ \\
\hline$<$ SKNN, EACImpute $>$ & $(0 / 2 / 0)$ & $(1 / 1 / 0)$ & $(1 / 1 / 0)$ & $(1 / 1 / 0)$ \\
\hline$<$ IKNNImpute, KMI $>$ & $(2 / 0 / 0)$ & $(2 / 0 / 0)$ & $(2 / 0 / 0)$ & $(2 / 0 / 0)$ \\
\hline$<$ IKNNImpute, EACImpute $>$ & $(0 / 2 / 0)$ & $(1 / 1 / 0)$ & $(1 / 1 / 0)$ & $(2 / 0 / 0)$ \\
\hline$<$ KMI, EACImpute $>$ & $(0 / 1 / 1)$ & $(0 / 2 / 0)$ & $(0 / 0 / 2)$ & $(0 / 0 / 2)$ \\
\hline
\end{tabular}

Tabela A.2: Resultados da comparação pareada (Vitórias/Empates/derrotas), em relação às diferenças estatísticas em cada taxa de ausentes $-\overline{E Q_{N}}$ para base de dados Íris. 


\begin{tabular}{|c|c|c|c|c|c|c|c|c|}
\hline $\begin{array}{c}\text { Taxa de } \\
\text { Ausentes }\end{array}$ & Algoritmos & Média Geral & Média Majority & KNNImpute & SKNN & IKNNImpute & KMI & EACImpute \\
\hline \multirow{7}{*}{$10 \%$} & Média Geral & $(-/-/-)$ & $(0 / 0 / 2)$ & $(0 / 0 / 2)$ & $(0 / 0 / 2)$ & $(0 / 0 / 2)$ & $(0 / 0 / 2)$ & $(0 / 0 / 2)$ \\
\hline & Média Majority & $(2 / 0 / 0)$ & $(-/-/-)$ & $(2 / 0 / 0)$ & $(2 / 0 / 0)$ & $(2 / 0 / 0)$ & $(2 / 0 / 0)$ & $(2 / 0 / 0)$ \\
\hline & KNNImpute & $(2 / 0 / 0)$ & $(0 / 0 / 2)$ & $(-/-/-)$ & $(2 / 0 / 0)$ & $(2 / 0 / 0)$ & $(2 / 0 / 0)$ & $(2 / 0 / 0)$ \\
\hline & SKNN & $(2 / 0 / 0)$ & $(0 / 0 / 2)$ & $(0 / 0 / 2)$ & $(-/-/-)$ & $(2 / 0 / 0)$ & $(1 / 0 / 1)$ & $(2 / 0 / 0)$ \\
\hline & IKNNImpute & $(2 / 0 / 0)$ & $(0 / 0 / 2)$ & $(0 / 0 / 2)$ & $(0 / 0 / 2)$ & $(-/-/-)$ & $(1 / 0 / 1)$ & $(2 / 0 / 0)$ \\
\hline & KMI & $(2 / 0 / 0)$ & $(0 / 0 / 2)$ & $(0 / 0 / 2)$ & $(1 / 0 / 1)$ & $(1 / 0 / 1)$ & $(-/-/-)$ & $(2 / 0 / 0)$ \\
\hline & EACImpute & $(2 / 0 / 0)$ & $(0 / 0 / 2)$ & $(0 / 0 / 2)$ & $(0 / 0 / 2)$ & $(0 / 0 / 2)$ & $(0 / 0 / 2)$ & $(-/-/-)$ \\
\hline \multirow{7}{*}{$30 \%$} & Média Geral & $(-/-/-)$ & $(0 / 0 / 2)$ & $(0 / 0 / 2)$ & $(0 / 0 / 2)$ & $(0 / 0 / 2)$ & $(0 / 0 / 2)$ & $(0 / 0 / 2)$ \\
\hline & Média Majority & $(2 / 0 / 0)$ & $(-/-/-)$ & $(2 / 0 / 0)$ & $(2 / 0 / 0)$ & $(2 / 0 / 0)$ & $(1 / 0 / 1)$ & $(2 / 0 / 0)$ \\
\hline & KNNImpute & $(2 / 0 / 0)$ & $(0 / 0 / 2)$ & $(-/-/-)$ & $(2 / 0 / 0)$ & $(1 / 0 / 1)$ & $(0 / 0 / 2)$ & $(2 / 0 / 0)$ \\
\hline & SKNN & $(2 / 0 / 0)$ & $(0 / 0 / 2)$ & $(0 / 0 / 2)$ & $(-/-/-)$ & $(0 / 0 / 2)$ & $(0 / 0 / 2)$ & $(1 / 0 / 1)$ \\
\hline & IKNNImpute & $(2 / 0 / 0)$ & $(0 / 0 / 2)$ & $(1 / 0 / 1)$ & $(2 / 0 / 0)$ & $(-/-/-)$ & $(1 / 0 / 1)$ & $(1 / 1 / 0)$ \\
\hline & KMI & $(2 / 0 / 0)$ & $(1 / 0 / 1)$ & $(2 / 0 / 0)$ & $(2 / 0 / 0)$ & $(1 / 0 / 1)$ & $(-/-/-)$ & $(2 / 0 / 0)$ \\
\hline & EACImpute & $(2 / 0 / 0)$ & $(0 / 0 / 2)$ & $(0 / 0 / 2)$ & $(1 / 0 / 1)$ & $(0 / 1 / 1)$ & $(0 / 0 / 2)$ & $(-/-/-)$ \\
\hline \multirow{7}{*}{$50 \%$} & Média Geral & $(-/-/-)$ & $(0 / 0 / 2)$ & $(0 / 0 / 2)$ & $(0 / 0 / 2)$ & $(0 / 0 / 2)$ & $(0 / 0 / 2)$ & $(0 / 0 / 2)$ \\
\hline & Média Majority & $(2 / 0 / 0)$ & $(-/-/-)$ & $(1 / 0 / 1)$ & $(1 / 0 / 1)$ & $(2 / 0 / 0)$ & $(2 / 0 / 0)$ & $(1 / 0 / 1)$ \\
\hline & KNNImpute & $(2 / 0 / 0)$ & $(1 / 0 / 1)$ & $(-/-/-)$ & $(2 / 0 / 0)$ & $(2 / 0 / 0)$ & $(1 / 1 / 0)$ & $(1 / 0 / 1)$ \\
\hline & SKNN & $(2 / 0 / 0)$ & $(1 / 0 / 1)$ & $(0 / 0 / 2)$ & $(-/-/-)$ & $(2 / 0 / 0)$ & $(1 / 0 / 1)$ & $(0 / 0 / 2)$ \\
\hline & IKNNImpute & $(2 / 0 / 0)$ & $(0 / 0 / 2)$ & $(0 / 0 / 2)$ & $(0 / 0 / 2)$ & $(-/-/-)$ & $(0 / 0 / 2)$ & $(0 / 0 / 2)$ \\
\hline & KMI & $(2 / 0 / 0)$ & $(0 / 0 / 2)$ & $(0 / 1 / 1)$ & $(1 / 0 / 1)$ & $(2 / 0 / 0)$ & $(-/-/-)$ & $(0 / 0 / 2)$ \\
\hline & EACImpute & $(2 / 0 / 0)$ & $(1 / 0 / 1)$ & $(1 / 0 / 1)$ & $(2 / 0 / 0)$ & $(2 / 0 / 0)$ & $(2 / 0 / 0)$ & $(-/-/-)$ \\
\hline \multirow{7}{*}{$70 \%$} & Média Geral & $(-/-/-)$ & $(0 / 0 / 2)$ & $(0 / 0 / 2)$ & $(0 / 0 / 2)$ & $(0 / 0 / 2)$ & $(0 / 0 / 2)$ & $(0 / 0 / 2)$ \\
\hline & Média Majority & $(2 / 0 / 0)$ & $(-/-/-)$ & $(1 / 0 / 1)$ & $(1 / 0 / 1)$ & $(1 / 0 / 1)$ & $(1 / 0 / 1)$ & $(2 / 0 / 0)$ \\
\hline & KNNImpute & $(2 / 0 / 0)$ & $(1 / 0 / 1)$ & $(-/-/-)$ & $(2 / 0 / 0)$ & $(2 / 0 / 0)$ & $(2 / 0 / 0)$ & $(1 / 0 / 1)$ \\
\hline & SKNN & $(2 / 0 / 0)$ & $(1 / 0 / 1)$ & $(0 / 0 / 2)$ & $(-/-/-)$ & $(2 / 0 / 0)$ & $(2 / 0 / 0)$ & $(1 / 0 / 1)$ \\
\hline & IKNNImpute & $(2 / 0 / 0)$ & $(1 / 0 / 1)$ & $(0 / 0 / 2)$ & $(0 / 0 / 2)$ & $(-/-/-)$ & $(0 / 0 / 2)$ & $(1 / 0 / 1)$ \\
\hline & KMI & $(2 / 0 / 0)$ & $(1 / 0 / 1)$ & $(0 / 0 / 2)$ & $(0 / 0 / 2)$ & $(2 / 0 / 0)$ & $(-/-/-)$ & $(1 / 0 / 1)$ \\
\hline & EACImpute & $(2 / 0 / 0)$ & $(0 / 0 / 2)$ & $(1 / 0 / 1)$ & $(1 / 0 / 1)$ & $(1 / 0 / 1)$ & $(1 / 0 / 1)$ & $(-/-/-)$ \\
\hline
\end{tabular}

Tabela A.3: Resultados da comparação pareada (Vitórias/Empates/derrotas), dos "ranks" médios em cada taxa de ausentes - viés na classificação para base de dados Íris.

\begin{tabular}{|c|c|c|c|c|}
\hline Pares de Algoritmos & $10 \%$ & $30 \%$ & $50 \%$ & $70 \%$ \\
\hline$<$ Média Geral, Média Majority $>$ & $(0 / 0 / 2)$ & $(0 / 0 / 2)$ & $(0 / 0 / 2)$ & $(0 / 0 / 2)$ \\
\hline$<$ Média Geral, KNNImpute $>$ & $(0 / 0 / 2)$ & $(0 / 0 / 2)$ & $(0 / 0 / 2)$ & $(0 / 1 / 1)$ \\
\hline$<$ Média Geral, SKNN $>$ & $(0 / 0 / 2)$ & $(0 / 0 / 2)$ & $(0 / 0 / 2)$ & $(0 / 1 / 1)$ \\
\hline$<$ Média Geral, IKNNImpute $>$ & $(0 / 0 / 2)$ & $(0 / 0 / 2)$ & $(0 / 0 / 2)$ & $(0 / 1 / 1)$ \\
\hline$<$ Média Geral, KMI $>$ & $(0 / 0 / 2)$ & $(0 / 0 / 2)$ & $(0 / 0 / 2)$ & $(0 / 1 / 1)$ \\
\hline$<$ Média Geral, EACImpute $>$ & $(0 / 0 / 2)$ & $(0 / 0 / 2)$ & $(0 / 0 / 2)$ & $(0 / 0 / 2)$ \\
\hline$<$ Média Majority, KNNImpute $>$ & $(0 / 2 / 0)$ & $(0 / 2 / 0)$ & $(0 / 2 / 0)$ & $(0 / 2 / 0)$ \\
\hline <Média Majority, SKNN> & $(0 / 2 / 0)$ & $(0 / 2 / 0)$ & $(0 / 2 / 0)$ & $(0 / 2 / 0)$ \\
\hline$<$ Média Majority, IKNNImpute $>$ & $(0 / 2 / 0)$ & $(0 / 2 / 0)$ & $(0 / 2 / 0)$ & $(0 / 2 / 0)$ \\
\hline$<$ Média Majority, KMI $>$ & $(0 / 2 / 0)$ & $(0 / 2 / 0)$ & $(0 / 2 / 0)$ & $(0 / 2 / 0)$ \\
\hline$<$ Média Majority, EACImpute $>$ & $(0 / 2 / 0)$ & $(0 / 2 / 0)$ & $(0 / 2 / 0)$ & $(0 / 2 / 0)$ \\
\hline$<$ KNNImpute, SKNN $>$ & $(0 / 2 / 0)$ & $(0 / 2 / 0)$ & $(0 / 2 / 0)$ & $(0 / 2 / 0)$ \\
\hline$<$ KNNImpute, IKNNImpute $>$ & $(0 / 2 / 0)$ & $(0 / 2 / 0)$ & $(0 / 2 / 0)$ & $(0 / 2 / 0)$ \\
\hline$<$ KNNImpute, KMI $>$ & $(0 / 2 / 0)$ & $(0 / 2 / 0)$ & $(0 / 2 / 0)$ & $(0 / 2 / 0)$ \\
\hline$<$ KNNImpute, EACImpute $>$ & $(0 / 2 / 0)$ & $(0 / 2 / 0)$ & $(0 / 2 / 0)$ & $(0 / 2 / 0)$ \\
\hline$<$ SKNN, IKNNImpute $>$ & $(0 / 2 / 0)$ & $(0 / 2 / 0)$ & $(0 / 2 / 0)$ & $(0 / 2 / 0)$ \\
\hline$<$ SKNN, KMI $>$ & $(0 / 2 / 0)$ & $(0 / 2 / 0)$ & $(0 / 2 / 0)$ & $(0 / 2 / 0)$ \\
\hline$<$ SKNN, EACImpute $>$ & $(0 / 2 / 0)$ & $(0 / 2 / 0)$ & $(0 / 2 / 0)$ & $(0 / 2 / 0)$ \\
\hline$<$ IKNNImpute, KMI $>$ & $(0 / 2 / 0)$ & $(0 / 2 / 0)$ & $(0 / 2 / 0)$ & $(0 / 2 / 0)$ \\
\hline$<$ IKNNImpute, EACImpute $>$ & $(0 / 2 / 0)$ & $(0 / 2 / 0)$ & $(0 / 2 / 0)$ & $(0 / 2 / 0)$ \\
\hline$<$ KMI, EACImpute $>$ & $(0 / 2 / 0)$ & $(0 / 2 / 0)$ & $(0 / 2 / 0)$ & $(0 / 2 / 0)$ \\
\hline
\end{tabular}

Tabela A.4: Resultados da comparação pareada (Vitórias/Empates/derrotas), em relação às diferenças estatísticas em cada taxa de ausentes - viés na classificação para base de dados Íris. 
A Apêndice

\section{A.1.2 Base de Dados “Glass Identification”}

\begin{tabular}{|c|c|c|c|c|c|c|c|c|}
\hline $\begin{array}{l}\text { Taxa de } \\
\text { Ausentes }\end{array}$ & Algoritmos & Média Geral & Média Majority & KNNImpute & SKNN & IKNNImpute & KMI & EACImpute \\
\hline \multirow{7}{*}{$10 \%$} & Média Geral & $(-/-/-)$ & $(4 / 0 / 0)$ & $(2 / 0 / 2)$ & $(2 / 0 / 2)$ & $(2 / 0 / 2)$ & $(4 / 0 / 0)$ & $(2 / 0 / 2)$ \\
\hline & Média Majority & $(0 / 0 / 4)$ & $(-/-/-)$ & $(0 / 0 / 4)$ & $(0 / 0 / 4)$ & $(0 / 0 / 4)$ & $(4 / 0 / 0)$ & $(0 / 0 / 4)$ \\
\hline & KNNImpute & $(2 / 0 / 2)$ & $(4 / 0 / 0)$ & $(-/-/-)$ & $(1 / 0 / 3)$ & $(1 / 0 / 3)$ & $(4 / 0 / 0)$ & $(0 / 0 / 4)$ \\
\hline & SKNN & $(2 / 0 / 2)$ & $(4 / 0 / 0)$ & $(3 / 0 / 1)$ & $(-/-/-)$ & $(1 / 0 / 3)$ & $(4 / 0 / 0)$ & $(1 / 0 / 3)$ \\
\hline & IKNNImpute & $(2 / 0 / 2)$ & $(4 / 0 / 0)$ & $(3 / 0 / 1)$ & $(3 / 0 / 1)$ & $(-/-/-)$ & $(4 / 0 / 0)$ & $(1 / 0 / 3)$ \\
\hline & KMI & $(0 / 0 / 4)$ & $(0 / 0 / 4)$ & $(0 / 0 / 4)$ & $(0 / 0 / 4)$ & $(0 / 0 / 4)$ & $(-/-/-)$ & $(0 / 0 / 4)$ \\
\hline & EACImpute & $(2 / 0 / 2)$ & $(4 / 0 / 0)$ & $(4 / 0 / 0)$ & $(3 / 0 / 1)$ & $(3 / 0 / 1)$ & $(4 / 0 / 0)$ & $(-/-/-)$ \\
\hline \multirow{7}{*}{$30 \%$} & Média Geral & $(-/-/-)$ & $(4 / 0 / 0)$ & $(1 / 0 / 3)$ & $(1 / 0 / 3)$ & $(1 / 0 / 3)$ & $(4 / 0 / 0)$ & $(1 / 0 / 3)$ \\
\hline & Média Majority & $(0 / 0 / 4)$ & $(-/-/-)$ & $(0 / 0 / 4)$ & $(0 / 0 / 4)$ & $(0 / 0 / 4)$ & $(4 / 0 / 0)$ & $(0 / 0 / 4)$ \\
\hline & KNNImpute & $(3 / 0 / 1)$ & $(4 / 0 / 0)$ & $(-/-/-)$ & $(3 / 0 / 1)$ & $(1 / 0 / 3)$ & $(4 / 0 / 0)$ & $(1 / 0 / 3)$ \\
\hline & SKNN & $(3 / 0 / 1)$ & $(4 / 0 / 0)$ & $(1 / 0 / 3)$ & $(-/-/-)$ & $(0 / 0 / 4)$ & $(4 / 0 / 0)$ & $(1 / 0 / 3)$ \\
\hline & IKNNImpute & $(3 / 0 / 1)$ & $(4 / 0 / 0)$ & $(3 / 0 / 1)$ & $(4 / 0 / 0)$ & $(-/-/-)$ & $(4 / 0 / 0)$ & $(3 / 0 / 1)$ \\
\hline & KMI & $(0 / 0 / 4)$ & $(0 / 0 / 4)$ & $(0 / 0 / 4)$ & $(0 / 0 / 4)$ & $(0 / 0 / 4)$ & $(-/-/-)$ & $(0 / 0 / 4)$ \\
\hline & EACImpute & $(3 / 0 / 1)$ & $(4 / 0 / 0)$ & $(3 / 0 / 1)$ & $(3 / 0 / 1)$ & $(1 / 0 / 3)$ & $(4 / 0 / 0)$ & $(-/-/-)$ \\
\hline \multirow{7}{*}{$50 \%$} & Média Geral & $(-/-/-)$ & $(3 / 0 / 1)$ & $(1 / 0 / 3)$ & $(1 / 0 / 3)$ & $(0 / 0 / 4)$ & $(4 / 0 / 0)$ & $(0 / 0 / 4)$ \\
\hline & Média Majority & $(1 / 0 / 3)$ & $(-/-/-)$ & $(0 / 0 / 4)$ & $(0 / 0 / 4)$ & $(0 / 0 / 4)$ & $(4 / 0 / 0)$ & $(0 / 0 / 4)$ \\
\hline & KNNImpute & $(3 / 0 / 1)$ & $(4 / 0 / 0)$ & $(-/-/-)$ & $(3 / 0 / 1)$ & $(2 / 0 / 2)$ & $(4 / 0 / 0)$ & $(3 / 0 / 1)$ \\
\hline & SKNN & $(3 / 0 / 1)$ & $(4 / 0 / 0)$ & $(1 / 0 / 3)$ & $(-/-/-)$ & $(0 / 0 / 4)$ & $(4 / 0 / 0)$ & $(1 / 0 / 3)$ \\
\hline & IKNNImpute & $(4 / 0 / 0)$ & $(4 / 0 / 0)$ & $(2 / 0 / 2)$ & $(4 / 0 / 0)$ & $(-/-/-)$ & $(4 / 0 / 0)$ & $(2 / 1 / 1)$ \\
\hline & KMI & $(0 / 0 / 4)$ & $(0 / 0 / 4)$ & $(0 / 0 / 4)$ & $(0 / 0 / 4)$ & $(0 / 0 / 4)$ & $(-/-/-)$ & $(0 / 0 / 4)$ \\
\hline & EACImpute & $(4 / 0 / 0)$ & $(4 / 0 / 0)$ & $(1 / 0 / 3)$ & $(3 / 0 / 1)$ & $(1 / 1 / 2)$ & $(4 / 0 / 0)$ & $(-/-/-)$ \\
\hline \multirow{7}{*}{$70 \%$} & Média Geral & $(-/-/-)$ & $(1 / 0 / 3)$ & $(0 / 0 / 4)$ & $(0 / 0 / 4)$ & $(0 / 0 / 4)$ & $(4 / 0 / 0)$ & $(0 / 0 / 4)$ \\
\hline & Média Majority & $(3 / 0 / 1)$ & $(-/-/-)$ & $(0 / 0 / 4)$ & $(0 / 0 / 4)$ & $(0 / 0 / 4)$ & $(4 / 0 / 0)$ & $(0 / 0 / 4)$ \\
\hline & KNNImpute & $(4 / 0 / 0)$ & $(4 / 0 / 0)$ & $(-/-/-)$ & $(3 / 0 / 1)$ & $(1 / 0 / 3)$ & $(4 / 0 / 0)$ & $(0 / 0 / 4)$ \\
\hline & SKNN & $(4 / 0 / 0)$ & $(4 / 0 / 0)$ & $(1 / 0 / 3)$ & $(-/-/-)$ & $(0 / 0 / 4)$ & $(4 / 0 / 0)$ & $(1 / 0 / 3)$ \\
\hline & IKNNImpute & $(4 / 0 / 0)$ & $(4 / 0 / 0)$ & $(3 / 0 / 1)$ & $(4 / 0 / 0)$ & $(-/-/-)$ & $(4 / 0 / 0)$ & $(1 / 1 / 2)$ \\
\hline & KMI & $(0 / 0 / 4)$ & $(0 / 0 / 4)$ & $(0 / 0 / 4)$ & $(0 / 0 / 4)$ & $(0 / 0 / 4)$ & $(-/-/-)$ & $(0 / 0 / 4)$ \\
\hline & EACImpute & $(4 / 0 / 0)$ & $(4 / 0 / 0)$ & $(4 / 0 / 0)$ & $(3 / 0 / 1)$ & $(2 / 1 / 1)$ & $(4 / 0 / 0)$ & $(-/-/-)$ \\
\hline
\end{tabular}

Tabela A.5: Resultados da comparação pareada (Vitórias/Empates/derrotas), dos "ranks" médios em cada taxa de ausentes - viés para base de dados Glass Identification.

\begin{tabular}{|c|c|c|c|c|}
\hline Pares de Algoritmos & $10 \%$ & $30 \%$ & $50 \%$ & $70 \%$ \\
\hline$<$ Média Geral, Média Majority $>$ & $(2 / 2 / 0)$ & $(1 / 3 / 0)$ & $(1 / 3 / 0)$ & $(1 / 2 / 1)$ \\
\hline$<$ Média Geral, KNNImpute $>$ & $(1 / 3 / 0)$ & $(1 / 1 / 2)$ & $(0 / 1 / 3)$ & $(0 / 1 / 3)$ \\
\hline$<$ Média Geral, SKNN $>$ & $(1 / 3 / 0)$ & $(1 / 2 / 1)$ & $(0 / 1 / 3)$ & $(0 / 1 / 3)$ \\
\hline$<$ Média Geral, IKNNImpute $>$ & $(1 / 3 / 0)$ & $(1 / 1 / 2)$ & $(0 / 1 / 3)$ & $(0 / 1 / 3)$ \\
\hline$<$ Média Geral, KMI $>$ & $(4 / 0 / 0)$ & $(3 / 1 / 0)$ & $(2 / 2 / 0)$ & $(1 / 3 / 0)$ \\
\hline$<$ Média Geral, EACImpute $>$ & $(0 / 4 / 0)$ & $(1 / 1 / 2)$ & $(0 / 1 / 3)$ & $(0 / 1 / 3)$ \\
\hline$<$ Média Majority, KNNImpute $>$ & $(0 / 2 / 2)$ & $(0 / 0 / 4)$ & $(0 / 0 / 4)$ & $(0 / 1 / 3)$ \\
\hline$<$ Média Majority, SKNN $>$ & $(0 / 1 / 3)$ & $(0 / 0 / 4)$ & $(0 / 0 / 4)$ & $(0 / 0 / 4)$ \\
\hline$<$ Média Majority, IKNNImpute $>$ & $(0 / 1 / 3)$ & $(0 / 0 / 4)$ & $(0 / 0 / 4)$ & $(0 / 0 / 4)$ \\
\hline$<$ Média Majority, KMI $>$ & $(0 / 4 / 0)$ & $(0 / 4 / 0)$ & $(1 / 3 / 0)$ & $(3 / 1 / 0)$ \\
\hline$<$ Média Majority, EACImpute $>$ & $(0 / 1 / 3)$ & $(0 / 0 / 4)$ & $(0 / 1 / 3)$ & $(0 / 0 / 4)$ \\
\hline$<$ KNNImpute, SKNN $>$ & $(0 / 4 / 0)$ & $(0 / 4 / 0)$ & $(0 / 4 / 0)$ & $(0 / 4 / 0)$ \\
\hline$<$ KNNImpute, IKNNImpute $>$ & $(0 / 4 / 0)$ & $(0 / 4 / 0)$ & $(0 / 4 / 0)$ & $(0 / 3 / 1)$ \\
\hline$<$ KNNImpute, KMI $>$ & $(4 / 0 / 0)$ & $(4 / 0 / 0)$ & $(4 / 0 / 0)$ & $(4 / 0 / 0)$ \\
\hline$<$ KNNImpute, EACImpute $>$ & $(0 / 4 / 0)$ & $(0 / 4 / 0)$ & $(0 / 4 / 0)$ & $(0 / 4 / 0)$ \\
\hline$<$ SKNN, IKNNImpute $>$ & $(0 / 4 / 0)$ & $(0 / 4 / 0)$ & $(0 / 4 / 0)$ & $(0 / 4 / 0)$ \\
\hline$<$ SKNN, KMI $>$ & $(4 / 0 / 0)$ & $(4 / 0 / 0)$ & $(4 / 0 / 0)$ & $(4 / 0 / 0)$ \\
\hline$<$ SKNN, EACImpute $>$ & $(0 / 4 / 0)$ & $(0 / 4 / 0)$ & $(0 / 4 / 0)$ & $(0 / 4 / 0)$ \\
\hline$<$ IKNNImpute, KMI $>$ & $(4 / 0 / 0)$ & $(4 / 0 / 0)$ & $(4 / 0 / 0)$ & $(4 / 0 / 0)$ \\
\hline$<$ IKNNImpute, EACImpute $>$ & $(0 / 4 / 0)$ & $(0 / 4 / 0)$ & $(1 / 3 / 0)$ & $(0 / 4 / 0)$ \\
\hline$<$ KMI, EACImpute $>$ & $(0 / 0 / 4)$ & $(0 / 0 / 4)$ & $(0 / 0 / 4)$ & $(0 / 0 / 4)$ \\
\hline & & & &
\end{tabular}

Tabela A.6: Resultados da comparação pareada (Vitórias/Empates/derrotas), em relação às diferenças estatísticas em cada taxa de ausentes $-\overline{E Q_{N}}$ para a base de dados Glass Identification. 


\begin{tabular}{|c|c|c|c|c|c|c|c|c|}
\hline $\begin{array}{c}\text { Taxa de } \\
\text { Ausentes }\end{array}$ & Algoritmos & Média Geral & Média Majority & KNNImpute & SKNN & IKNNImpute & KMI & EACImpute \\
\hline \multirow{7}{*}{$10 \%$} & Média Geral & $(-/-/-)$ & $(0 / 0 / 4)$ & $(0 / 0 / 4)$ & $(0 / 0 / 4)$ & $(0 / 0 / 4)$ & $(0 / 0 / 4)$ & $(0 / 0 / 4)$ \\
\hline & Média Majority & $(4 / 0 / 0)$ & $(-/-/-)$ & $(0 / 0 / 4)$ & $(0 / 0 / 4)$ & $(0 / 0 / 4)$ & $(0 / 0 / 4)$ & $(0 / 0 / 4)$ \\
\hline & KNNImpute & $(4 / 0 / 0)$ & $(4 / 0 / 0)$ & $(-/-/-)$ & $(2 / 0 / 2)$ & $(2 / 0 / 2)$ & $(4 / 0 / 0)$ & $(4 / 0 / 0)$ \\
\hline & SKNN & $(4 / 0 / 0)$ & $(4 / 0 / 0)$ & $(2 / 0 / 2)$ & $(-/-/-)$ & $(1 / 1 / 2)$ & $(4 / 0 / 0)$ & $(4 / 0 / 0)$ \\
\hline & IKNNImpute & $(4 / 0 / 0)$ & $(4 / 0 / 0)$ & $(2 / 0 / 2)$ & $(2 / 1 / 1)$ & $(-/-/-)$ & $(4 / 0 / 0)$ & $(3 / 0 / 1)$ \\
\hline & KMI & $(4 / 0 / 0)$ & $(4 / 0 / 0)$ & $(0 / 0 / 4)$ & $(0 / 0 / 4)$ & $(0 / 0 / 4)$ & $(-/-/-)$ & $(1 / 0 / 3)$ \\
\hline & EACImpute & $(4 / 0 / 0)$ & $(4 / 0 / 0)$ & $(0 / 0 / 4)$ & $(0 / 0 / 4)$ & $(1 / 0 / 3)$ & $(3 / 0 / 1)$ & $(-/-/-)$ \\
\hline \multirow{7}{*}{$30 \%$} & Média Geral & $(-/-/-)$ & $(0 / 0 / 4)$ & $(0 / 0 / 4)$ & $(0 / 0 / 4)$ & $(0 / 0 / 4)$ & $(0 / 0 / 4)$ & $(0 / 0 / 4)$ \\
\hline & Média Majority & $(4 / 0 / 0)$ & $(-/-/-)$ & $(0 / 0 / 4)$ & $(0 / 0 / 4)$ & $(0 / 0 / 4)$ & $(0 / 0 / 4)$ & $(0 / 0 / 4)$ \\
\hline & KNNImpute & $(4 / 0 / 0)$ & $(4 / 0 / 0)$ & $(-/-/-)$ & $(1 / 0 / 3)$ & $(0 / 0 / 4)$ & $(4 / 0 / 0)$ & $(3 / 0 / 1)$ \\
\hline & SKNN & $(4 / 0 / 0)$ & $(4 / 0 / 0)$ & $(3 / 0 / 1)$ & $(-/-/-)$ & $(0 / 0 / 4)$ & $(4 / 0 / 0)$ & $(3 / 0 / 1)$ \\
\hline & IKNNImpute & $(4 / 0 / 0)$ & $(4 / 0 / 0)$ & $(4 / 0 / 0)$ & $(4 / 0 / 0)$ & $(-/-/-)$ & $(4 / 0 / 0)$ & $(3 / 0 / 1)$ \\
\hline & KMI & $(4 / 0 / 0)$ & $(4 / 0 / 0)$ & $(0 / 0 / 4)$ & $(0 / 0 / 4)$ & $(0 / 0 / 4)$ & $(-/-/-)$ & $(2 / 0 / 2)$ \\
\hline & EACImpute & $(4 / 0 / 0)$ & $(4 / 0 / 0)$ & $(1 / 0 / 3)$ & $(1 / 0 / 3)$ & $(1 / 0 / 3)$ & $(2 / 0 / 2)$ & $(-/-/-)$ \\
\hline \multirow{7}{*}{$50 \%$} & Média Geral & $(-/-/-)$ & $(0 / 0 / 4)$ & $(0 / 0 / 4)$ & $(0 / 0 / 4)$ & $(0 / 0 / 4)$ & $(0 / 0 / 4)$ & $(0 / 0 / 4)$ \\
\hline & Média Majority & $(4 / 0 / 0)$ & $(-/-/-)$ & $(0 / 0 / 4)$ & $(0 / 0 / 4)$ & $(0 / 0 / 4)$ & $(0 / 0 / 4)$ & $(2 / 0 / 2)$ \\
\hline & KNNImpute & $(4 / 0 / 0)$ & $(4 / 0 / 0)$ & $(-/-/-)$ & $(0 / 0 / 4)$ & $(0 / 0 / 4)$ & $(1 / 0 / 3)$ & $(3 / 0 / 1)$ \\
\hline & SKNN & $(4 / 0 / 0)$ & $(4 / 0 / 0)$ & $(4 / 0 / 0)$ & $(-/-/-)$ & $(0 / 0 / 4)$ & $(3 / 0 / 1)$ & $(4 / 0 / 0)$ \\
\hline & IKNNImpute & $(4 / 0 / 0)$ & $(4 / 0 / 0)$ & $(4 / 0 / 0)$ & $(4 / 0 / 0)$ & $(-/-/-)$ & $(3 / 0 / 1)$ & $(4 / 0 / 0)$ \\
\hline & KMI & $(4 / 0 / 0)$ & $(4 / 0 / 0)$ & $(3 / 0 / 1)$ & $(1 / 0 / 3)$ & $(1 / 0 / 3)$ & $(-/-/-)$ & $(4 / 0 / 0)$ \\
\hline & EACImpute & $(4 / 0 / 0)$ & $(2 / 0 / 2)$ & $(1 / 0 / 3)$ & $(0 / 0 / 4)$ & $(0 / 0 / 4)$ & $(0 / 0 / 4)$ & $(-/-/-)$ \\
\hline \multirow{7}{*}{$70 \%$} & Média Geral & $(-/-/-)$ & $(0 / 0 / 4)$ & $(0 / 0 / 4)$ & $(0 / 0 / 4)$ & $(0 / 0 / 4)$ & $(0 / 0 / 4)$ & $(0 / 0 / 4)$ \\
\hline & Média Majority & $(4 / 0 / 0)$ & $(-/-/-)$ & $(0 / 0 / 4)$ & $(0 / 0 / 4)$ & $(0 / 0 / 4)$ & $(2 / 0 / 2)$ & $(1 / 0 / 3)$ \\
\hline & KNNImpute & $(4 / 0 / 0)$ & $(4 / 0 / 0)$ & $(-/-/-)$ & $(0 / 0 / 4)$ & $(1 / 0 / 3)$ & $(3 / 0 / 1)$ & $(4 / 0 / 0)$ \\
\hline & SKNN & $(4 / 0 / 0)$ & $(4 / 0 / 0)$ & $(4 / 0 / 0)$ & $(-/-/-)$ & $(1 / 0 / 3)$ & $(4 / 0 / 0)$ & $(4 / 0 / 0)$ \\
\hline & IKNNImpute & $(4 / 0 / 0)$ & $(4 / 0 / 0)$ & $(3 / 0 / 1)$ & $(3 / 0 / 1)$ & $(-/-/-)$ & $(4 / 0 / 0)$ & $(4 / 0 / 0)$ \\
\hline & KMI & $(4 / 0 / 0)$ & $(2 / 0 / 2)$ & $(1 / 0 / 3)$ & $(0 / 0 / 4)$ & $(0 / 0 / 4)$ & $(-/-/-)$ & $(1 / 0 / 3)$ \\
\hline & EACImpute & $(4 / 0 / 0)$ & $(3 / 0 / 1)$ & $(0 / 0 / 4)$ & $(0 / 0 / 4)$ & $(0 / 0 / 4)$ & $(3 / 0 / 1)$ & $(-/-/-)$ \\
\hline
\end{tabular}

Tabela A.7: Resultados da comparação pareada (Vitórias/Empates/derrotas), dos "ranks" médios em cada taxa de ausentes - viés na classificação para a base de dados Glass Identification.

\begin{tabular}{|c|c|c|c|c|}
\hline Pares de Algoritmos & $10 \%$ & $30 \%$ & $50 \%$ & $70 \%$ \\
\hline$<$ Média Geral,Média Majority $>$ & $(0 / 0 / 3)$ & $(0 / 0 / 4)$ & $(0 / 0 / 4)$ & $(0 / 0 / 4)$ \\
\hline$<$ Média Geral,KNNImpute $>$ & $(0 / 0 / 4)$ & $(0 / 0 / 4)$ & $(0 / 0 / 4)$ & $(0 / 0 / 4)$ \\
\hline$<$ Média Geral,SKNN $>$ & $(0 / 0 / 4)$ & $(0 / 0 / 4)$ & $(0 / 0 / 4)$ & $(0 / 0 / 4)$ \\
\hline$<$ Média Geral,IKNNImpute $>$ & $(0 / 0 / 4)$ & $(0 / 0 / 4)$ & $(0 / 0 / 4)$ & $(0 / 0 / 4)$ \\
\hline$<$ Média Geral,KMI $>$ & $(0 / 0 / 4)$ & $(0 / 0 / 4)$ & $(0 / 0 / 4)$ & $(0 / 1 / 3)$ \\
\hline$<$ Média Geral,EACImpute $>$ & $(0 / 0 / 4)$ & $(0 / 0 / 4)$ & $(0 / 0 / 4)$ & $(0 / 1 / 3)$ \\
\hline$<$ Média Majority,KNNImpute $>$ & $(0 / 1 / 3)$ & $(0 / 1 / 3)$ & $(0 / 3 / 1)$ & $(0 / 4 / 0)$ \\
\hline$<$ Média Majority,SKNN $>$ & $(0 / 2 / 2)$ & $(0 / 0 / 4)$ & $(0 / 1 / 3)$ & $(0 / 3 / 1)$ \\
\hline$<$ Média Majority,IKNNImpute $>$ & $(0 / 2 / 2)$ & $(0 / 0 / 4)$ & $(0 / 1 / 3)$ & $(0 / 2 / 2)$ \\
\hline$<$ Média Majority,KMI $>$ & $(0 / 4 / 0)$ & $(0 / 4 / 0)$ & $(0 / 3 / 1)$ & $(0 / 4 / 0)$ \\
\hline$<$ Média Majority,EACImpute $>$ & $(0 / 4 / 0)$ & $(0 / 3 / 1)$ & $(0 / 4 / 0)$ & $(0 / 4 / 0)$ \\
\hline$<$ KNNImpute,SKNN $>$ & $(0 / 4 / 0)$ & $(0 / 4 / 0)$ & $(0 / 4 / 0)$ & $(0 / 4 / 0)$ \\
\hline$<$ KNNImpute,IKNNImpute $>$ & $(0 / 4 / 0)$ & $(0 / 3 / 1)$ & $(0 / 3 / 1)$ & $(0 / 4 / 0)$ \\
\hline$<$ KNNImpute,KMI $>$ & $(0 / 4 / 0)$ & $(1 / 3 / 0)$ & $(0 / 4 / 0)$ & $(0 / 4 / 0)$ \\
\hline$<$ KNNImpute,EACImpute $>$ & $(0 / 4 / 0)$ & $(0 / 4 / 0)$ & $(1 / 3 / 0)$ & $(0 / 4 / 0)$ \\
\hline$<$ SKNN,IKNNImpute $>$ & $(0 / 4 / 0)$ & $(0 / 4 / 0)$ & $(0 / 4 / 0)$ & $(0 / 4 / 0)$ \\
\hline$<$ SKNN,KMI $>$ & $(0 / 4 / 0)$ & $(2 / 2 / 0)$ & $(0 / 4 / 0)$ & $(0 / 4 / 0)$ \\
\hline$<$ SKNN,EACImpute $>$ & $(0 / 4 / 0)$ & $(1 / 3 / 0)$ & $(3 / 1 / 0)$ & $(0 / 4 / 0)$ \\
\hline$<$ IKNNImpute,KMI $>$ & $(1 / 3 / 0)$ & $(3 / 1 / 0)$ & $(0 / 4 / 0)$ & $(1 / 3 / 0)$ \\
\hline$<$ IKNNImpute,EACImpute $>$ & $(1 / 3 / 0)$ & $(3 / 1 / 0)$ & $(3 / 1 / 0)$ & $(0 / 4 / 0)$ \\
\hline$<$ KMI,EACImpute $>$ & $(0 / 4 / 0)$ & $(0 / 3 / 1)$ & $(2 / 2 / 0)$ & $(0 / 4 / 0)$ \\
\hline
\end{tabular}

Tabela A.8: Resultados da comparação pareada (Vitórias/Empates/derrotas), em relação às diferenças estatísticas observadas em cada taxa de ausentes - viés na classificação para a base de dados Glass Identification. 
A Apêndice

\section{A.1.3 Base de Dados "Yeast"}

\begin{tabular}{|c|c|c|c|c|c|c|c|c|}
\hline $\begin{array}{l}\text { Taxa de } \\
\text { Ausentes }\end{array}$ & Algoritmos & Média Geral & Média Majority & KNNImpute & SKNN & IKNNImpute & KMI & EACImpute \\
\hline \multirow{7}{*}{$10 \%$} & Média Geral & $(-/-/-)$ & $(4 / 0 / 0)$ & $(2 / 0 / 2)$ & $(2 / 0 / 2)$ & $(2 / 0 / 2)$ & $(4 / 0 / 0)$ & $(2 / 0 / 2)$ \\
\hline & Média Majority & $(0 / 0 / 4)$ & $(-/-/-)$ & $(0 / 0 / 4)$ & $(0 / 0 / 4)$ & $(0 / 0 / 4)$ & $(4 / 0 / 0)$ & $(0 / 0 / 4)$ \\
\hline & KNNImpute & $(2 / 0 / 2)$ & $(4 / 0 / 0)$ & $(-/-/-)$ & $(1 / 0 / 3)$ & $(1 / 0 / 3)$ & $(4 / 0 / 0)$ & $(0 / 0 / 4)$ \\
\hline & SKNN & $(2 / 0 / 2)$ & $(4 / 0 / 0)$ & $(3 / 0 / 1)$ & $(-/-/-)$ & $(1 / 0 / 3)$ & $(4 / 0 / 0)$ & $(1 / 0 / 3)$ \\
\hline & IKNNImpute & $(2 / 0 / 2)$ & $(4 / 0 / 0)$ & $(3 / 0 / 1)$ & $(3 / 0 / 1)$ & $(-/-/-)$ & $(4 / 0 / 0)$ & $(1 / 0 / 3)$ \\
\hline & KMI & $(0 / 0 / 4)$ & $(0 / 0 / 4)$ & $(0 / 0 / 4)$ & $(0 / 0 / 4)$ & $(0 / 0 / 4)$ & $(-/-/-)$ & $(0 / 0 / 4)$ \\
\hline & EACImpute & $(2 / 0 / 2)$ & $(4 / 0 / 0)$ & $(4 / 0 / 0)$ & $(3 / 0 / 1)$ & $(3 / 0 / 1)$ & $(4 / 0 / 0)$ & $(-/-/-)$ \\
\hline \multirow{7}{*}{$30 \%$} & Média Geral & $(-/-/-)$ & $(4 / 0 / 0)$ & $(1 / 0 / 3)$ & $(1 / 0 / 3)$ & $(1 / 0 / 3)$ & $(4 / 0 / 0)$ & $(1 / 0 / 3)$ \\
\hline & Média Majority & $(0 / 0 / 4)$ & $(-/-/-)$ & $(0 / 0 / 4)$ & $(0 / 0 / 4)$ & $(0 / 0 / 4)$ & $(4 / 0 / 0)$ & $(0 / 0 / 4)$ \\
\hline & KNNImpute & $(3 / 0 / 1)$ & $(4 / 0 / 0)$ & $(-/-/-)$ & $(3 / 0 / 1)$ & $(1 / 0 / 3)$ & $(4 / 0 / 0)$ & $(1 / 0 / 3)$ \\
\hline & SKNN & $(3 / 0 / 1)$ & $(4 / 0 / 0)$ & $(1 / 0 / 3)$ & $(-/-/-)$ & $(0 / 0 / 4)$ & $(4 / 0 / 0)$ & $(1 / 0 / 3)$ \\
\hline & IKNNImpute & $(3 / 0 / 1)$ & $(4 / 0 / 0)$ & $(3 / 0 / 1)$ & $(4 / 0 / 0)$ & $(-/-/-)$ & $(4 / 0 / 0)$ & $(3 / 0 / 1)$ \\
\hline & KMI & $(0 / 0 / 4)$ & $(0 / 0 / 4)$ & $(0 / 0 / 4)$ & $(0 / 0 / 4)$ & $(0 / 0 / 4)$ & $(-/-/-)$ & $(0 / 0 / 4)$ \\
\hline & EACImpute & $(3 / 0 / 1)$ & $(4 / 0 / 0)$ & $(3 / 0 / 1)$ & $(3 / 0 / 1)$ & $(1 / 0 / 3)$ & $(4 / 0 / 0)$ & $(-/-/-)$ \\
\hline \multirow{7}{*}{$50 \%$} & Média Geral & $(-/-/-)$ & $(3 / 0 / 1)$ & $(1 / 0 / 3)$ & $(1 / 0 / 3)$ & $(0 / 0 / 4)$ & $(4 / 0 / 0)$ & $(0 / 0 / 4)$ \\
\hline & Média Majority & $(1 / 0 / 3)$ & $(-/-/-)$ & $(0 / 0 / 4)$ & $(0 / 0 / 4)$ & $(0 / 0 / 4)$ & $(4 / 0 / 0)$ & $(0 / 0 / 4)$ \\
\hline & KNNImpute & $(3 / 0 / 1)$ & $(4 / 0 / 0)$ & $(-/-/-)$ & $(3 / 0 / 1)$ & $(2 / 0 / 2)$ & $(4 / 0 / 0)$ & $(3 / 0 / 1)$ \\
\hline & SKNN & $(3 / 0 / 1)$ & $(4 / 0 / 0)$ & $(1 / 0 / 3)$ & $(-/-/-)$ & $(0 / 0 / 4)$ & $(4 / 0 / 0)$ & $(1 / 0 / 3)$ \\
\hline & IKNNImpute & $(4 / 0 / 0)$ & $(4 / 0 / 0)$ & $(2 / 0 / 2)$ & $(4 / 0 / 0)$ & $(-/-/-)$ & $(4 / 0 / 0)$ & $(2 / 1 / 1)$ \\
\hline & KMI & $(0 / 0 / 4)$ & $(0 / 0 / 4)$ & $(0 / 0 / 4)$ & $(0 / 0 / 4)$ & $(0 / 0 / 4)$ & $(-/-/-)$ & $(0 / 0 / 4)$ \\
\hline & EACImpute & $(4 / 0 / 0)$ & $(4 / 0 / 0)$ & $(1 / 0 / 3)$ & $(3 / 0 / 1)$ & $(1 / 1 / 2)$ & $(4 / 0 / 0)$ & $(-/-/-)$ \\
\hline \multirow{7}{*}{$70 \%$} & Média Geral & $(-/-/-)$ & $(1 / 0 / 3)$ & $(0 / 0 / 4)$ & $(0 / 0 / 4)$ & $(0 / 0 / 4)$ & $(4 / 0 / 0)$ & $(0 / 0 / 4)$ \\
\hline & Média Majority & $(3 / 0 / 1)$ & $(-/-/-)$ & $(0 / 0 / 4)$ & $(0 / 0 / 4)$ & $(0 / 0 / 4)$ & $(4 / 0 / 0)$ & $(0 / 0 / 4)$ \\
\hline & KNNImpute & $(4 / 0 / 0)$ & $(4 / 0 / 0)$ & $(-/-/-)$ & $(3 / 0 / 1)$ & $(1 / 0 / 3)$ & $(4 / 0 / 0)$ & $(0 / 0 / 4)$ \\
\hline & SKNN & $(4 / 0 / 0)$ & $(4 / 0 / 0)$ & $(1 / 0 / 3)$ & $(-/-/-)$ & $(0 / 0 / 4)$ & $(4 / 0 / 0)$ & $(1 / 0 / 3)$ \\
\hline & IKNNImpute & $(4 / 0 / 0)$ & $(4 / 0 / 0)$ & $(3 / 0 / 1)$ & $(4 / 0 / 0)$ & $(-/-/-)$ & $(4 / 0 / 0)$ & $(1 / 1 / 2)$ \\
\hline & KMI & $(0 / 0 / 4)$ & $(0 / 0 / 4)$ & $(0 / 0 / 4)$ & $(0 / 0 / 4)$ & $(0 / 0 / 4)$ & $(-/-/-)$ & $(0 / 0 / 4)$ \\
\hline & EACImpute & $(4 / 0 / 0)$ & $(4 / 0 / 0)$ & $(4 / 0 / 0)$ & $(3 / 0 / 1)$ & $(2 / 1 / 1)$ & $(4 / 0 / 0)$ & $(-/-/-)$ \\
\hline
\end{tabular}

Tabela A.9: Resultados da comparação pareada (Vitórias/Empates/derrotas) dos "ranks" médios em cada taxa de ausentes $-\overline{E Q_{N}}$ para a base de dados Yeast.

\begin{tabular}{|c|c|c|c|c|}
\hline Pares de Algoritmos & $10 \%$ & $30 \%$ & $50 \%$ & $70 \%$ \\
\hline$<$ Média Geral, Média Majority $>$ & $(2 / 2 / 0)$ & $(1 / 3 / 0)$ & $(1 / 3 / 0)$ & $(1 / 2 / 1)$ \\
\hline$<$ Média Geral, KNNImpute $>$ & $(1 / 3 / 0)$ & $(1 / 1 / 2)$ & $(0 / 1 / 3)$ & $(0 / 1 / 3)$ \\
\hline$<$ Média Geral, SKNN $>$ & $(1 / 3 / 0)$ & $(1 / 2 / 1)$ & $(0 / 1 / 3)$ & $(0 / 1 / 3)$ \\
\hline$<$ Média Geral, IKNNImpute $>$ & $(1 / 3 / 0)$ & $(1 / 1 / 2)$ & $(0 / 1 / 3)$ & $(0 / 1 / 3)$ \\
\hline$<$ Média Geral, KMI $>$ & $(4 / 0 / 0)$ & $(3 / 1 / 0)$ & $(2 / 2 / 0)$ & $(1 / 3 / 0)$ \\
\hline$<$ Média Geral, EACImpute $>$ & $(0 / 4 / 0)$ & $(1 / 1 / 2)$ & $(0 / 1 / 3)$ & $(0 / 1 / 3)$ \\
\hline$<$ Média Majority, KNNImpute $>$ & $(0 / 2 / 2)$ & $(0 / 0 / 4)$ & $(0 / 0 / 4)$ & $(0 / 1 / 3)$ \\
\hline$<$ Média Majority, SKNN $>$ & $(0 / 1 / 3)$ & $(0 / 0 / 4)$ & $(0 / 0 / 4)$ & $(0 / 0 / 4)$ \\
\hline$<$ Média Majority, IKNNImpute $>$ & $(0 / 1 / 3)$ & $(0 / 0 / 4)$ & $(0 / 0 / 4)$ & $(0 / 0 / 4)$ \\
\hline$<$ Média Majority, KMI $>$ & $(0 / 4 / 0)$ & $(0 / 4 / 0)$ & $(1 / 3 / 0)$ & $(3 / 1 / 0)$ \\
\hline$<$ Média Majority, EACImpute $>$ & $(0 / 1 / 3)$ & $(0 / 0 / 4)$ & $(0 / 1 / 3)$ & $(0 / 0 / 4)$ \\
\hline$<$ KNNImpute, SKNN $>$ & $(0 / 4 / 0)$ & $(0 / 4 / 0)$ & $(0 / 4 / 0)$ & $(0 / 4 / 0)$ \\
\hline$<$ KNNImpute, IKNNImpute $>$ & $(0 / 4 / 0)$ & $(0 / 4 / 0)$ & $(0 / 4 / 0)$ & $(0 / 3 / 1)$ \\
\hline$<$ KNNImpute, KMI $>$ & $(4 / 0 / 0)$ & $(4 / 0 / 0)$ & $(4 / 0 / 0)$ & $(4 / 0 / 0)$ \\
\hline$<$ KNNImpute, EACImpute $>$ & $(0 / 4 / 0)$ & $(0 / 4 / 0)$ & $(0 / 4 / 0)$ & $(0 / 4 / 0)$ \\
\hline$<$ SKNN, IKNNImpute $>$ & $(0 / 4 / 0)$ & $(0 / 4 / 0)$ & $(0 / 4 / 0)$ & $(0 / 4 / 0)$ \\
\hline$<$ SKNN, KMI $>$ & $(4 / 0 / 0)$ & $(4 / 0 / 0)$ & $(4 / 0 / 0)$ & $(4 / 0 / 0)$ \\
\hline$<$ SKNN, EACImpute $>$ & $(0 / 4 / 0)$ & $(0 / 4 / 0)$ & $(0 / 4 / 0)$ & $(0 / 4 / 0)$ \\
\hline$<$ IKNNImpute, KMI $>$ & $(4 / 0 / 0)$ & $(4 / 0 / 0)$ & $(4 / 0 / 0)$ & $(4 / 0 / 0)$ \\
\hline$<$ IKNNImpute, EACImpute $>$ & $(0 / 4 / 0)$ & $(0 / 4 / 0)$ & $(1 / 3 / 0)$ & $(0 / 4 / 0)$ \\
\hline$<$ KMI, EACImpute $>$ & $(0 / 0 / 4)$ & $(0 / 0 / 4)$ & $(0 / 0 / 4)$ & $(0 / 0 / 4)$ \\
\hline & & & &
\end{tabular}

Tabela A.10: Resultados da comparação pareada (Vitórias/Empates/derrotas) em relação às diferenças estatísticas observadas em cada taxa de ausentes $-\overline{E Q_{N}}$ para a base de dados Yeast. 


\begin{tabular}{|c|c|c|c|c|c|c|c|c|}
\hline $\begin{array}{c}\text { Taxa de } \\
\text { Ausentes }\end{array}$ & Algoritmos & Média Geral & Média Majority & KNNImpute & SKNN & IKNNImpute & KMI & EACImpute \\
\hline \multirow{7}{*}{$10 \%$} & Média Geral & $(-/-/-)$ & $(0 / 0 / 4)$ & $(0 / 0 / 4)$ & $(1 / 0 / 3)$ & $(1 / 0 / 3)$ & $(0 / 0 / 4)$ & $(0 / 0 / 4)$ \\
\hline & Média Majority & $(4 / 0 / 0)$ & $(-/-/-)$ & $(3 / 0 / 1)$ & $(4 / 0 / 0)$ & $(4 / 0 / 0)$ & $(2 / 0 / 2)$ & $(4 / 0 / 0)$ \\
\hline & KNNImpute & $(4 / 0 / 0)$ & $(1 / 0 / 3)$ & $(-/-/-)$ & $(4 / 0 / 0)$ & $(4 / 0 / 0)$ & $(0 / 0 / 4)$ & $(2 / 0 / 2)$ \\
\hline & SKNN & $(3 / 0 / 1)$ & $(0 / 0 / 4)$ & $(0 / 0 / 4)$ & $(-/-/-)$ & $(4 / 0 / 0)$ & $(0 / 0 / 4)$ & $(1 / 0 / 3)$ \\
\hline & IKNNImpute & $(3 / 0 / 1)$ & $(0 / 0 / 4)$ & $(0 / 0 / 4)$ & $(0 / 0 / 4)$ & $(-/-/-)$ & $(0 / 0 / 4)$ & $(0 / 0 / 4)$ \\
\hline & KMI & $(4 / 0 / 0)$ & $(2 / 0 / 2)$ & $(4 / 0 / 0)$ & $(4 / 0 / 0)$ & $(4 / 0 / 0)$ & $(-/-/-)$ & $(3 / 0 / 1)$ \\
\hline & EACImpute & $(4 / 0 / 0)$ & $(0 / 0 / 4)$ & $(2 / 0 / 2)$ & $(3 / 0 / 1)$ & $(4 / 0 / 0)$ & $(1 / 0 / 3)$ & $(-/-/-)$ \\
\hline \multirow{7}{*}{$30 \%$} & Média Geral & $(-/-/-)$ & $(0 / 0 / 4)$ & $(1 / 0 / 3)$ & $(1 / 0 / 3)$ & $(1 / 0 / 3)$ & $(0 / 0 / 4)$ & $(0 / 0 / 4)$ \\
\hline & Média Majority & $(4 / 0 / 0)$ & $(-/-/-)$ & $(1 / 0 / 3)$ & $(2 / 0 / 2)$ & $(3 / 0 / 1)$ & $(0 / 0 / 4)$ & $(0 / 0 / 4)$ \\
\hline & KNNImpute & $(3 / 0 / 1)$ & $(3 / 0 / 1)$ & $(-/-/-)$ & $(4 / 0 / 0)$ & $(4 / 0 / 0)$ & $(3 / 0 / 1)$ & $(2 / 0 / 2)$ \\
\hline & SKNN & $(3 / 0 / 1)$ & $(2 / 0 / 2)$ & $(0 / 0 / 4)$ & $(-/-/-)$ & $(4 / 0 / 0)$ & $(1 / 0 / 3)$ & $(2 / 0 / 2)$ \\
\hline & IKNNImpute & $(3 / 0 / 1)$ & $(1 / 0 / 3)$ & $(0 / 0 / 4)$ & $(0 / 0 / 4)$ & $(-/-/-)$ & $(0 / 0 / 4)$ & $(1 / 0 / 3)$ \\
\hline & KMI & $(4 / 0 / 0)$ & $(4 / 0 / 0)$ & $(1 / 0 / 3)$ & $(3 / 0 / 1)$ & $(4 / 0 / 0)$ & $(-/-/-)$ & $(2 / 0 / 2)$ \\
\hline & EACImpute & $(4 / 0 / 0)$ & $(4 / 0 / 0)$ & $(2 / 0 / 2)$ & $(2 / 0 / 2)$ & $(3 / 0 / 1)$ & $(2 / 0 / 2)$ & $(-/-/-)$ \\
\hline \multirow{7}{*}{$50 \%$} & Média Geral & $(-/-/-)$ & $(0 / 0 / 4)$ & $(0 / 0 / 4)$ & $(1 / 0 / 3)$ & $(2 / 0 / 2)$ & $(0 / 0 / 4)$ & $(0 / 0 / 4)$ \\
\hline & Média Majority & $(4 / 0 / 0)$ & $(-/-/-)$ & $(0 / 0 / 4)$ & $(2 / 0 / 2)$ & $(3 / 0 / 1)$ & $(1 / 0 / 3)$ & $(0 / 0 / 4)$ \\
\hline & KNNImpute & $(4 / 0 / 0)$ & $(4 / 0 / 0)$ & $(-/-/-)$ & $(4 / 0 / 0)$ & $(4 / 0 / 0)$ & $(4 / 0 / 0)$ & $(3 / 0 / 1)$ \\
\hline & SKNN & $(3 / 0 / 1)$ & $(2 / 0 / 2)$ & $(0 / 0 / 4)$ & $(-/-/-)$ & $(4 / 0 / 0)$ & $(2 / 1 / 1)$ & $(2 / 0 / 2)$ \\
\hline & IKNNImpute & $(2 / 0 / 2)$ & $(1 / 0 / 3)$ & $(0 / 0 / 4)$ & $(0 / 0 / 4)$ & $(-/-/-)$ & $(0 / 0 / 4)$ & $(0 / 0 / 4)$ \\
\hline & KMI & $(4 / 0 / 0)$ & $(3 / 0 / 1)$ & $(0 / 0 / 4)$ & $(1 / 1 / 2)$ & $(4 / 0 / 0)$ & $(-/-/-)$ & $(1 / 0 / 3)$ \\
\hline & EACImpute & $(4 / 0 / 0)$ & $(4 / 0 / 0)$ & $(1 / 0 / 3)$ & $(2 / 0 / 2)$ & $(4 / 0 / 0)$ & $(3 / 0 / 1)$ & $(-/-/-)$ \\
\hline \multirow{7}{*}{$70 \%$} & Média Geral & $(-/-/-)$ & $(0 / 0 / 4)$ & $(0 / 0 / 4)$ & $(2 / 0 / 2)$ & $(3 / 0 / 1)$ & $(0 / 0 / 4)$ & $(0 / 0 / 4)$ \\
\hline & Média Majority & $(4 / 0 / 0)$ & $(-/-/-)$ & $(1 / 0 / 3)$ & $(2 / 0 / 2)$ & $(4 / 0 / 0)$ & $(1 / 1 / 2)$ & $(0 / 0 / 4)$ \\
\hline & KNNImpute & $(4 / 0 / 0)$ & $(3 / 0 / 1)$ & $(-/-/-)$ & $(4 / 0 / 0)$ & $(4 / 0 / 0)$ & $(4 / 0 / 0)$ & $(3 / 0 / 1)$ \\
\hline & SKNN & $(2 / 0 / 2)$ & $(2 / 0 / 2)$ & $(0 / 0 / 4)$ & $(-/-/-)$ & $(4 / 0 / 0)$ & $(1 / 0 / 3)$ & $(2 / 0 / 2)$ \\
\hline & IKNNImpute & $(1 / 0 / 3)$ & $(0 / 0 / 4)$ & $(0 / 0 / 4)$ & $(0 / 0 / 4)$ & $(-/-/-)$ & $(0 / 0 / 4)$ & $(0 / 0 / 4)$ \\
\hline & KMI & $(4 / 0 / 0)$ & $(2 / 1 / 1)$ & $(0 / 0 / 4)$ & $(3 / 0 / 1)$ & $(4 / 0 / 0)$ & $(-/-/-)$ & $(2 / 0 / 2)$ \\
\hline & EACImpute & $(4 / 0 / 0)$ & $(4 / 0 / 0)$ & $(1 / 0 / 3)$ & $(2 / 0 / 2)$ & $(4 / 0 / 0)$ & $(2 / 0 / 2)$ & $(-/-/-)$ \\
\hline
\end{tabular}

Tabela A.11: Resultados da comparação pareada (Vitórias/Empates/derrotas), dos "ranks" médios em cada taxa de ausentes - viés na classificação para a base de dados Yeast.

\begin{tabular}{|c|c|c|c|c|}
\hline Pares de Algoritmos & $10 \%$ & $30 \%$ & $50 \%$ & $70 \%$ \\
\hline$<$ Média Geral,Média Majority $>$ & $(0 / 1 / 3)$ & $(0 / 2 / 2)$ & $(0 / 3 / 1)$ & $(0 / 3 / 1)$ \\
\hline$<$ Média Geral,KNNImpute $>$ & $(0 / 2 / 2)$ & $(0 / 1 / 3)$ & $(0 / 2 / 2)$ & $(0 / 1 / 3)$ \\
\hline$<$ Média Geral,SKNN $>$ & $(0 / 3 / 1)$ & $(0 / 2 / 2)$ & $(0 / 2 / 2)$ & $(0 / 3 / 1)$ \\
\hline$<$ Média Geral,IKNNImpute $>$ & $(0 / 4 / 0)$ & $(0 / 3 / 1)$ & $(0 / 3 / 1)$ & $(0 / 4 / 0)$ \\
\hline$<$ Média Geral,KMI $>$ & $(0 / 2 / 2)$ & $(0 / 1 / 3)$ & $(0 / 2 / 2)$ & $(0 / 3 / 1)$ \\
\hline$<$ Média Geral,EACImpute $>$ & $(0 / 2 / 2)$ & $(0 / 1 / 3)$ & $(0 / 2 / 2)$ & $(0 / 3 / 1)$ \\
\hline$<$ Média Majority,KNNImpute $>$ & $(0 / 4 / 0)$ & $(0 / 2 / 2)$ & $(0 / 2 / 2)$ & $(0 / 2 / 2)$ \\
\hline$<$ Média Majority,SKNN $>$ & $(0 / 4 / 0)$ & $(0 / 3 / 1)$ & $(0 / 4 / 0)$ & $(0 / 4 / 0)$ \\
\hline$<$ Média Majority,IKNNImpute $>$ & $(1 / 3 / 0)$ & $(0 / 4 / 0)$ & $(0 / 4 / 0)$ & $(0 / 4 / 0)$ \\
\hline$<$ Média Majority,KMI $>$ & $(0 / 4 / 0)$ & $(0 / 4 / 0)$ & $(0 / 4 / 0)$ & $(0 / 4 / 0)$ \\
\hline$<$ Média Majority,EACImpute $>$ & $(1 / 3 / 0)$ & $(0 / 4 / 0)$ & $(0 / 4 / 0)$ & $(0 / 4 / 0)$ \\
\hline$<$ KNNImpute,SKNN $>$ & $(0 / 4 / 0)$ & $(1 / 3 / 0)$ & $(1 / 3 / 0)$ & $(3 / 1 / 0)$ \\
\hline$<$ KNNImpute,IKNNImpute $>$ & $(1 / 3 / 0)$ & $(2 / 2 / 0)$ & $(3 / 1 / 0)$ & $(3 / 1 / 0)$ \\
\hline$<$ KNNImpute,KMI $>$ & $(0 / 4 / 0)$ & $(2 / 2 / 0)$ & $(2 / 2 / 0)$ & $(2 / 2 / 0)$ \\
\hline$<$ KNNImpute,EACImpute $>$ & $(1 / 3 / 0)$ & $(2 / 2 / 0)$ & $(2 / 2 / 0)$ & $(2 / 2 / 0)$ \\
\hline$<$ SKNN,IKNNImpute $>$ & $(0 / 4 / 0)$ & $(0 / 4 / 0)$ & $(0 / 4 / 0)$ & $(0 / 4 / 0)$ \\
\hline$<$ SKNN,KMI $>$ & $(0 / 3 / 1)$ & $(0 / 4 / 0)$ & $(0 / 4 / 0)$ & $(0 / 4 / 0)$ \\
\hline$<$ SKNN,EACImpute $>$ & $(0 / 4 / 0)$ & $(0 / 4 / 0)$ & $(0 / 4 / 0)$ & $(0 / 4 / 0)$ \\
\hline$<$ IKNNImpute,KMI $>$ & $(0 / 3 / 1)$ & $(0 / 4 / 0)$ & $(0 / 3 / 1)$ & $(0 / 4 / 0)$ \\
\hline$<$ IKNNImpute,EACImpute $>$ & $(0 / 4 / 0)$ & $(0 / 4 / 0)$ & $(0 / 2 / 2)$ & $(0 / 2 / 2)$ \\
\hline$<$ KMI,EACImpute $>$ & $(1 / 3 / 0)$ & $(0 / 4 / 0)$ & $(0 / 4 / 0)$ & $(0 / 4 / 0)$ \\
\hline
\end{tabular}

Tabela A.12: Resultados da comparação pareada (Vitórias/Empates/derrotas) em relação às diferenças estatísticas observadas em cada taxa de ausentes - viés na classificação para a base de dados Yeast. 
A Apêndice

\section{A.1.4 Base de Dados "Pen-Digts"}

\begin{tabular}{|c|c|c|c|c|c|c|c|c|}
\hline $\begin{array}{c}\text { Taxa de } \\
\text { Ausentes }\end{array}$ & Algoritmos & Média Geral & Média Majority & KNNImpute & SKNN & IKNNImpute & KMI & EACImpute \\
\hline \multirow{7}{*}{$10 \%$} & Média Geral & $(-/-/-)$ & $(0 / 0 / 8)$ & $(0 / 0 / 8)$ & $(0 / 0 / 8)$ & $(0 / 0 / 8)$ & $(0 / 0 / 8)$ & $(0 / 0 / 8)$ \\
\hline & Média Majority & $(8 / 0 / 0)$ & $(-/-/-)$ & $(0 / 0 / 8)$ & $(0 / 0 / 8)$ & $(0 / 0 / 8)$ & $(8 / 0 / 0)$ & $(0 / 0 / 8)$ \\
\hline & KNNImpute & $(8 / 0 / 0)$ & $(8 / 0 / 0)$ & $(-/-/-)$ & $(4 / 0 / 4)$ & $(4 / 0 / 4)$ & $(8 / 0 / 0)$ & $(8 / 0 / 0)$ \\
\hline & SKNN & $(8 / 0 / 0)$ & $(8 / 0 / 0)$ & $(4 / 0 / 4)$ & $(-/-/-)$ & $(4 / 0 / 4)$ & $(8 / 0 / 0)$ & $(8 / 0 / 0)$ \\
\hline & IKNNImpute & $(8 / 0 / 0)$ & $(8 / 0 / 0)$ & $(4 / 0 / 4)$ & $(4 / 0 / 4)$ & $(-/-/-)$ & $(8 / 0 / 0)$ & $(8 / 0 / 0)$ \\
\hline & KMI & $(8 / 0 / 0)$ & $(0 / 0 / 8)$ & $(0 / 0 / 8)$ & $(0 / 0 / 8)$ & $(0 / 0 / 8)$ & $(-/-/-)$ & $(0 / 0 / 8)$ \\
\hline & EACImpute & $(8 / 0 / 0)$ & $(8 / 0 / 0)$ & $(0 / 0 / 8)$ & $(0 / 0 / 8)$ & $(0 / 0 / 8)$ & $(8 / 0 / 0)$ & $(-/-/-)$ \\
\hline \multirow{7}{*}{$30 \%$} & Média Geral & $(-/-/-)$ & $(0 / 0 / 8)$ & $(0 / 0 / 8)$ & $(0 / 0 / 8)$ & $(0 / 0 / 8)$ & $(0 / 0 / 8)$ & $(0 / 0 / 8)$ \\
\hline & Média Majority & $(8 / 0 / 0)$ & $(-/-/-)$ & $(0 / 0 / 8)$ & $(0 / 0 / 8)$ & $(0 / 0 / 8)$ & $(8 / 0 / 0)$ & $(0 / 0 / 8)$ \\
\hline & KNNImpute & $(8 / 0 / 0)$ & $(8 / 0 / 0)$ & $(-/-/-)$ & $(4 / 0 / 4)$ & $(2 / 0 / 6)$ & $(8 / 0 / 0)$ & $(8 / 0 / 0)$ \\
\hline & SKNN & $(8 / 0 / 0)$ & $(8 / 0 / 0)$ & $(4 / 0 / 4)$ & $(-/-/-)$ & $(2 / 0 / 6)$ & $(8 / 0 / 0)$ & $(8 / 0 / 0)$ \\
\hline & IKNNImpute & $(8 / 0 / 0)$ & $(8 / 0 / 0)$ & $(6 / 0 / 2)$ & $(6 / 0 / 2)$ & $(-/-/-)$ & $(8 / 0 / 0)$ & $(8 / 0 / 0)$ \\
\hline & KMI & $(8 / 0 / 0)$ & $(0 / 0 / 8)$ & $(0 / 0 / 8)$ & $(0 / 0 / 8)$ & $(0 / 0 / 8)$ & $(-/-/-)$ & $(0 / 0 / 8)$ \\
\hline & EACImpute & $(8 / 0 / 0)$ & $(8 / 0 / 0)$ & $(0 / 0 / 8)$ & $(0 / 0 / 8)$ & $(0 / 0 / 8)$ & $(8 / 0 / 0)$ & $(-/-/-)$ \\
\hline \multirow{7}{*}{$50 \%$} & Média Geral & $(-/-/-)$ & $(0 / 0 / 8)$ & $(0 / 0 / 8)$ & $(0 / 0 / 8)$ & $(0 / 0 / 8)$ & $(0 / 0 / 8)$ & $(0 / 0 / 8)$ \\
\hline & Média Majority & $(8 / 0 / 0)$ & $(-/-/-)$ & $(0 / 0 / 8)$ & $(0 / 0 / 8)$ & $(0 / 0 / 8)$ & $(8 / 0 / 0)$ & $(0 / 0 / 8)$ \\
\hline & KNNImpute & $(8 / 0 / 0)$ & $(8 / 0 / 0)$ & $(-/-/-)$ & $(5 / 0 / 3)$ & $(2 / 0 / 6)$ & $(8 / 0 / 0)$ & $(8 / 0 / 0)$ \\
\hline & SKNN & $(8 / 0 / 0)$ & $(8 / 0 / 0)$ & $(3 / 0 / 5)$ & $(-/-/-)$ & $(0 / 0 / 8)$ & $(8 / 0 / 0)$ & $(8 / 0 / 0)$ \\
\hline & IKNNImpute & $(8 / 0 / 0)$ & $(8 / 0 / 0)$ & $(6 / 0 / 2)$ & $(8 / 0 / 0)$ & $(-/-/-)$ & $(8 / 0 / 0)$ & $(8 / 0 / 0)$ \\
\hline & KMI & $(8 / 0 / 0)$ & $(0 / 0 / 8)$ & $(0 / 0 / 8)$ & $(0 / 0 / 8)$ & $(0 / 0 / 8)$ & $(-/-/-)$ & $(0 / 0 / 8)$ \\
\hline & EACImpute & $(8 / 0 / 0)$ & $(8 / 0 / 0)$ & $(0 / 0 / 8)$ & $(0 / 0 / 8)$ & $(0 / 0 / 8)$ & $(8 / 0 / 0)$ & $(-/-/-)$ \\
\hline \multirow{7}{*}{$70 \%$} & Média Geral & $(-/-/-)$ & $(0 / 0 / 8)$ & $(0 / 0 / 8)$ & $(0 / 0 / 8)$ & $(0 / 0 / 8)$ & $(0 / 0 / 8)$ & $(0 / 0 / 8)$ \\
\hline & Média Majority & $(8 / 0 / 0)$ & $(-/-/-)$ & $(0 / 0 / 8)$ & $(0 / 0 / 8)$ & $(0 / 0 / 8)$ & $(8 / 0 / 0)$ & $(0 / 0 / 8)$ \\
\hline & KNNImpute & $(8 / 0 / 0)$ & $(8 / 0 / 0)$ & $(-/-/-)$ & $(5 / 0 / 3)$ & $(3 / 0 / 5)$ & $(8 / 0 / 0)$ & $(7 / 0 / 1)$ \\
\hline & SKNN & $(8 / 0 / 0)$ & $(8 / 0 / 0)$ & $(3 / 0 / 5)$ & $(-/-/-)$ & $(0 / 0 / 8)$ & $(8 / 0 / 0)$ & $(8 / 0 / 0)$ \\
\hline & IKNNImpute & $(8 / 0 / 0)$ & $(8 / 0 / 0)$ & $(5 / 0 / 3)$ & $(8 / 0 / 0)$ & $(-/-/-)$ & $(8 / 0 / 0)$ & $(8 / 0 / 0)$ \\
\hline & KMI & $(8 / 0 / 0)$ & $(0 / 0 / 8)$ & $(0 / 0 / 8)$ & $(0 / 0 / 8)$ & $(0 / 0 / 8)$ & $(-/-/-)$ & $(0 / 0 / 8)$ \\
\hline & EACImpute & $(8 / 0 / 0)$ & $(8 / 0 / 0)$ & $(1 / 0 / 7)$ & $(0 / 0 / 8)$ & $(0 / 0 / 8)$ & $(8 / 0 / 0)$ & $(-/-/-)$ \\
\hline
\end{tabular}

Tabela A.13: Resultados da comparação pareada (Vitórias/Empates/derrotas) em relação às diferenças estatísticas observadas em cada taxa de ausentes $-\overline{E Q_{N}}$ para a base de dados PenDigits.

\begin{tabular}{|c|c|c|c|c|}
\hline Pares de Algoritmos & $10 \%$ & $30 \%$ & $50 \%$ & $70 \%$ \\
\hline$<$ Média Geral, Média Majority $>$ & $(0 / 0 / 8)$ & $(0 / 0 / 8)$ & $(0 / 0 / 8)$ & $(0 / 0 / 8)$ \\
\hline$<$ Média Geral, KNNImpute $>$ & $(0 / 0 / 8)$ & $(0 / 0 / 8)$ & $(0 / 0 / 8)$ & $(0 / 0 / 8)$ \\
\hline$<$ Média Geral, SKNN $>$ & $(0 / 0 / 8)$ & $(0 / 0 / 8)$ & $(0 / 0 / 8)$ & $(0 / 0 / 8)$ \\
\hline$<$ Média Geral, IKNNImpute $>$ & $(0 / 0 / 8)$ & $(0 / 0 / 8)$ & $(0 / 0 / 8)$ & $(0 / 0 / 8)$ \\
\hline$<$ Média Geral, KMI $>$ & $(0 / 8 / 0)$ & $(0 / 8 / 0)$ & $(0 / 8 / 0)$ & $(0 / 8 / 0)$ \\
\hline$<$ Média Geral, EACImpute $>$ & $(0 / 0 / 8)$ & $(0 / 0 / 8)$ & $(0 / 0 / 8)$ & $(0 / 0 / 8)$ \\
\hline$<$ Média Majority, KNNImpute $>$ & $(0 / 0 / 8)$ & $(0 / 0 / 8)$ & $(0 / 0 / 8)$ & $(0 / 0 / 8)$ \\
\hline$<$ Média Majority, SKNN $>$ & $(0 / 0 / 8)$ & $(0 / 0 / 8)$ & $(0 / 0 / 8)$ & $(0 / 0 / 8)$ \\
\hline$<$ Média Majority, IKNNImpute $>$ & $(0 / 0 / 8)$ & $(0 / 0 / 8)$ & $(0 / 0 / 8)$ & $(0 / 0 / 8)$ \\
\hline$<$ Média Majority, KMI $>$ & $(0 / 8 / 0)$ & $(0 / 8 / 0)$ & $(0 / 8 / 0)$ & $(0 / 8 / 0)$ \\
\hline$<$ Média Majority, EACImpute $>$ & $(0 / 8 / 0)$ & $(0 / 8 / 0)$ & $(0 / 8 / 0)$ & $(0 / 7 / 1)$ \\
\hline$<$ KNNImpute, SKNN $>$ & $(0 / 5 / 3)$ & $(3 / 2 / 3)$ & $(2 / 5 / 1)$ & $(3 / 4 / 1)$ \\
\hline$<$ KNNImpute, IKNNImpute $>$ & $(0 / 5 / 3)$ & $(0 / 7 / 1)$ & $(0 / 5 / 3)$ & $(0 / 5 / 3)$ \\
\hline$<$ KNNImpute, KMI $>$ & $(8 / 0 / 0)$ & $(8 / 0 / 0)$ & $(8 / 0 / 0)$ & $(8 / 0 / 0)$ \\
\hline$<$ KNNImpute, EACImpute $>$ & $(5 / 3 / 0)$ & $(5 / 3 / 0)$ & $(5 / 3 / 0)$ & $(5 / 3 / 0)$ \\
\hline$<$ SKNN, IKNNImpute $>$ & $(0 / 8 / 0)$ & $(0 / 6 / 2)$ & $(0 / 6 / 2)$ & $(0 / 5 / 3)$ \\
\hline$<$ SKNN, KMI $>$ & $(8 / 0 / 0)$ & $(8 / 0 / 0)$ & $(8 / 0 / 0)$ & $(8 / 0 / 0)$ \\
\hline$<$ SKNN, EACImpute $>$ & $(8 / 0 / 0)$ & $(4 / 4 / 0)$ & $(3 / 5 / 0)$ & $(3 / 5 / 0)$ \\
\hline$<$ IKNNImpute, KMI $>$ & $(8 / 0 / 0)$ & $(8 / 0 / 0)$ & $(8 / 0 / 0)$ & $(8 / 0 / 0)$ \\
\hline$<$ IKNNImpute, EACImpute $>$ & $(8 / 0 / 0)$ & $(8 / 0 / 0)$ & $(8 / 0 / 0)$ & $(8 / 0 / 0)$ \\
\hline$<$ KMI, EACImpute $>$ & $(0 / 0 / 8)$ & $(0 / 0 / 8)$ & $(0 / 0 / 8)$ & $(0 / 0 / 8)$ \\
\hline & & & &
\end{tabular}

Tabela A.14: Resultados da comparação pareada (Vitórias/Empates/derrotas) em relação às diferenças estatísticas observadas em cada taxa de ausentes $-\overline{E Q_{N}}$ para a base de dados PenDigits. 


\begin{tabular}{|c|c|c|c|c|c|c|c|c|}
\hline $\begin{array}{c}\text { Taxa de } \\
\text { Ausentes }\end{array}$ & Algoritmos & Média Geral & Média Majority & KNNImpute & SKNN & IKNNImpute & KMI & EACImpute \\
\hline \multirow{7}{*}{$10 \%$} & Média Geral & $(-/-/-)$ & $(0 / 0 / 8)$ & $(0 / 0 / 8)$ & $(0 / 0 / 8)$ & $(0 / 0 / 8)$ & $(0 / 0 / 8)$ & $(0 / 0 / 8)$ \\
\hline & Média Majority & $(8 / 0 / 0)$ & $(-/-/-)$ & $(0 / 0 / 8)$ & $(0 / 0 / 8)$ & $(0 / 0 / 8)$ & $(0 / 0 / 8)$ & $(0 / 0 / 8)$ \\
\hline & KNNImpute & $(8 / 0 / 0)$ & $(8 / 0 / 0)$ & $(-/-/-)$ & $(3 / 1 / 4)$ & $(6 / 0 / 2)$ & $(8 / 0 / 0)$ & $(7 / 0 / 1)$ \\
\hline & SKNN & $(8 / 0 / 0)$ & $(8 / 0 / 0)$ & $(4 / 1 / 3)$ & $(-/-/-)$ & $(8 / 0 / 0)$ & $(7 / 0 / 1)$ & $(7 / 0 / 1)$ \\
\hline & IKNNImpute & $(8 / 0 / 0)$ & $(8 / 0 / 0)$ & $(2 / 0 / 6)$ & $(0 / 0 / 8)$ & $(-/-/-)$ & $(7 / 0 / 1)$ & $(7 / 0 / 1)$ \\
\hline & KMI & $(8 / 0 / 0)$ & $(8 / 0 / 0)$ & $(0 / 0 / 8)$ & $(1 / 0 / 7)$ & $(1 / 0 / 7)$ & $(-/-/-)$ & $(0 / 0 / 8)$ \\
\hline & EACImpute & $(8 / 0 / 0)$ & $(8 / 0 / 0)$ & $(1 / 0 / 7)$ & $(1 / 0 / 7)$ & $(1 / 0 / 7)$ & $(8 / 0 / 0)$ & $(-/-/-)$ \\
\hline \multirow{7}{*}{$30 \%$} & Média Geral & $(-/-/-)$ & $(0 / 0 / 8)$ & $(0 / 0 / 8)$ & $(0 / 0 / 8)$ & $(0 / 0 / 8)$ & $(0 / 0 / 8)$ & $(0 / 0 / 8)$ \\
\hline & Média Majority & $(8 / 0 / 0)$ & $(-/-/-)$ & $(0 / 0 / 8)$ & $(0 / 0 / 8)$ & $(0 / 0 / 8)$ & $(0 / 0 / 8)$ & $(0 / 0 / 8)$ \\
\hline & KNNImpute & $(8 / 0 / 0)$ & $(8 / 0 / 0)$ & $(-/-/-)$ & $(5 / 1 / 2)$ & $(8 / 0 / 0)$ & $(8 / 0 / 0)$ & $(8 / 0 / 0)$ \\
\hline & SKNN & $(8 / 0 / 0)$ & $(8 / 0 / 0)$ & $(2 / 1 / 5)$ & $(-/-/-)$ & $(8 / 0 / 0)$ & $(8 / 0 / 0)$ & $(7 / 0 / 1)$ \\
\hline & IKNNImpute & $(8 / 0 / 0)$ & $(8 / 0 / 0)$ & $(0 / 0 / 8)$ & $(0 / 0 / 8)$ & $(-/-/-)$ & $(7 / 0 / 1)$ & $(6 / 0 / 2)$ \\
\hline & KMI & $(8 / 0 / 0)$ & $(8 / 0 / 0)$ & $(0 / 0 / 8)$ & $(0 / 0 / 8)$ & $(1 / 0 / 7)$ & $(-/-/-)$ & $(0 / 0 / 8)$ \\
\hline & EACImpute & $(8 / 0 / 0)$ & $(8 / 0 / 0)$ & $(0 / 0 / 8)$ & $(1 / 0 / 7)$ & $(2 / 0 / 6)$ & $(8 / 0 / 0)$ & $(-/-/-)$ \\
\hline \multirow{7}{*}{$50 \%$} & Média Geral & $(-/-/-)$ & $(0 / 0 / 8)$ & $(0 / 0 / 8)$ & $(0 / 0 / 8)$ & $(0 / 0 / 8)$ & $(0 / 0 / 8)$ & $(0 / 0 / 8)$ \\
\hline & Média Majority & $(8 / 0 / 0)$ & $(-/-/-)$ & $(0 / 0 / 8)$ & $(0 / 0 / 8)$ & $(0 / 0 / 8)$ & $(0 / 0 / 8)$ & $(0 / 0 / 8)$ \\
\hline & KNNImpute & $(8 / 0 / 0)$ & $(8 / 0 / 0)$ & $(-/-/-)$ & $(7 / 0 / 1)$ & $(8 / 0 / 0)$ & $(8 / 0 / 0)$ & $(8 / 0 / 0)$ \\
\hline & SKNN & $(8 / 0 / 0)$ & $(8 / 0 / 0)$ & $(1 / 0 / 7)$ & $(-/-/-)$ & $(8 / 0 / 0)$ & $(8 / 0 / 0)$ & $(6 / 0 / 2)$ \\
\hline & IKNNImpute & $(8 / 0 / 0)$ & $(8 / 0 / 0)$ & $(0 / 0 / 8)$ & $(0 / 0 / 8)$ & $(-/-/-)$ & $(7 / 0 / 1)$ & $(3 / 0 / 5)$ \\
\hline & KMI & $(8 / 0 / 0)$ & $(8 / 0 / 0)$ & $(0 / 0 / 8)$ & $(0 / 0 / 8)$ & $(1 / 0 / 7)$ & $(-/-/-)$ & $(0 / 0 / 8)$ \\
\hline & EACImpute & $(8 / 0 / 0)$ & $(8 / 0 / 0)$ & $(0 / 0 / 8)$ & $(2 / 0 / 6)$ & $(5 / 0 / 3)$ & $(8 / 0 / 0)$ & $(-/-/-)$ \\
\hline \multirow{7}{*}{$70 \%$} & Média Geral & $(-/-/-)$ & $(0 / 0 / 8)$ & $(0 / 0 / 8)$ & $(0 / 0 / 8)$ & $(0 / 0 / 8)$ & $(0 / 0 / 8)$ & $(0 / 0 / 8)$ \\
\hline & Média Majority & $(8 / 0 / 0)$ & $(-/-/-)$ & $(0 / 0 / 8)$ & $(0 / 0 / 8)$ & $(1 / 0 / 7)$ & $(0 / 0 / 8)$ & $(0 / 0 / 8)$ \\
\hline & KNNImpute & $(8 / 0 / 0)$ & $(8 / 0 / 0)$ & $(-/-/-)$ & $(6 / 0 / 2)$ & $(8 / 0 / 0)$ & $(8 / 0 / 0)$ & $(4 / 2 / 2)$ \\
\hline & SKNN & $(8 / 0 / 0)$ & $(8 / 0 / 0)$ & $(2 / 0 / 6)$ & $(-/-/-)$ & $(8 / 0 / 0)$ & $(5 / 0 / 3)$ & $(4 / 0 / 4)$ \\
\hline & IKNNImpute & $(8 / 0 / 0)$ & $(7 / 0 / 1)$ & $(0 / 0 / 8)$ & $(0 / 0 / 8)$ & $(-/-/-)$ & $(4 / 0 / 4)$ & $(1 / 0 / 7)$ \\
\hline & KMI & $(8 / 0 / 0)$ & $(8 / 0 / 0)$ & $(0 / 0 / 8)$ & $(3 / 0 / 5)$ & $(4 / 0 / 4)$ & $(-/-/-)$ & $(0 / 0 / 8)$ \\
\hline & EACImpute & $(8 / 0 / 0)$ & $(8 / 0 / 0)$ & $(2 / 2 / 4)$ & $(4 / 0 / 4)$ & $(7 / 0 / 1)$ & $(8 / 0 / 0)$ & $(-/-/-)$ \\
\hline
\end{tabular}

Tabela A.15: Resultados da comparação pareada (Vitórias/Empates/derrotas) em relação aos valores médios de "ranks" em cada taxa de ausentes - viés na classificação para a base de dados Pen-Digits.

\begin{tabular}{|c|c|c|c|c|}
\hline Pares de Algoritmos & $10 \%$ & $30 \%$ & $50 \%$ & $70 \%$ \\
\hline$<$ Média Geral,Média Majority $>$ & $(0 / 7 / 1)$ & $(0 / 7 / 1)$ & $(0 / 7 / 1)$ & $(0 / 4 / 4)$ \\
\hline$<$ Média Geral,KNNImpute $>$ & $(0 / 0 / 8)$ & $(0 / 0 / 8)$ & $(0 / 0 / 8)$ & $(0 / 0 / 8)$ \\
\hline$<$ Média Geral,SKNN $>$ & $(0 / 0 / 8)$ & $(0 / 0 / 8)$ & $(0 / 0 / 8)$ & $(0 / 0 / 8)$ \\
\hline$<$ Média Geral,IKNNImpute $>$ & $(0 / 0 / 8)$ & $(0 / 0 / 8)$ & $(0 / 0 / 8)$ & $(0 / 0 / 8)$ \\
\hline$<$ Média Geral,KMI $>$ & $(0 / 0 / 8)$ & $(0 / 0 / 8)$ & $(0 / 0 / 8)$ & $(0 / 0 / 8)$ \\
\hline$<$ Média Geral,EACImpute $>$ & $(0 / 0 / 8)$ & $(0 / 0 / 8)$ & $(0 / 0 / 8)$ & $(0 / 0 / 8)$ \\
\hline$<$ Média Majority,KNNImpute $>$ & $(0 / 1 / 7)$ & $(0 / 1 / 7)$ & $(0 / 0 / 8)$ & $(0 / 1 / 7)$ \\
\hline$<$ Média Majority,SKNN $>$ & $(0 / 1 / 7)$ & $(0 / 1 / 7)$ & $(0 / 0 / 8)$ & $(0 / 1 / 7)$ \\
\hline$<$ Média Majority,IKNNImpute $>$ & $(0 / 1 / 7)$ & $(0 / 1 / 7)$ & $(0 / 1 / 7)$ & $(0 / 3 / 5)$ \\
\hline$<$ Média Majority,KMI $>$ & $(0 / 5 / 3)$ & $(0 / 1 / 1)$ & $(0 / 4 / 4)$ & $(0 / 2 / 6)$ \\
\hline$<$ Média Majority,EACImpute $>$ & $(0 / 1 / 7)$ & $(0 / 1 / 7)$ & $(0 / 0 / 8)$ & $(0 / 2 / 6)$ \\
\hline$<$ KNNImpute,SKNN $>$ & $(0 / 8 / 0)$ & $(0 / 8 / 0)$ & $(0 / 8 / 0)$ & $(0 / 8 / 0)$ \\
\hline$<$ KNNImpute,IKNNImpute $>$ & $(0 / 8 / 0)$ & $(0 / 8 / 0)$ & $(7 / 1 / 0)$ & $(2 / 6 / 0)$ \\
\hline$<$ KNNImpute,KMI $>$ & $(6 / 2 / 0)$ & $(7 / 1 / 0)$ & $(0 / 8 / 0)$ & $(0 / 8 / 0)$ \\
\hline$<$ KNNImpute,EACImpute $>$ & $(2 / 6 / 0)$ & $(1 / 7 / 0)$ & $(0 / 8 / 0)$ & $(0 / 8 / 0)$ \\
\hline$<$ SKNN,IKNNImpute $>$ & $(0 / 8 / 0)$ & $(0 / 8 / 0)$ & $(0 / 8 / 0)$ & $(0 / 8 / 0)$ \\
\hline$<$ SKNN,KMI $>$ & $(6 / 2 / 0)$ & $(7 / 1 / 0)$ & $(6 / 2 / 0)$ & $(0 / 8 / 0)$ \\
\hline$<$ SKNN,EACImpute $>$ & $(2 / 6 / 0)$ & $(1 / 7 / 0)$ & $(0 / 8 / 0)$ & $(0 / 8 / 0)$ \\
\hline$<$ IKNNImpute,KMI $>$ & $(5 / 3 / 0)$ & $(7 / 1 / 0)$ & $(2 / 6 / 0)$ & $(0 / 7 / 1)$ \\
\hline$<$ IKNNImpute,EACImpute $>$ & $(1 / 7 / 0)$ & $(0 / 8 / 0)$ & $(0 / 8 / 0)$ & $(0 / 6 / 2)$ \\
\hline$<$ KMI,EACImpute $>$ & $(0 / 7 / 1)$ & $(0 / 6 / 2)$ & $(0 / 5 / 3)$ & $(0 / 8 / 0)$ \\
\hline & & & &
\end{tabular}

Tabela A.16: Resultados da comparação pareada (Vitórias/Empates/derrotas) em relação às diferenças estatísticas observadas em cada taxa de ausentes - viés na classificação para a base de dados Pen-Digits. 
A Apêndice

\section{A.1.5 Base de Dados "Segmentation"}

\begin{tabular}{|c|c|c|c|c|c|c|c|c|}
\hline $\begin{array}{c}\text { Taxa de } \\
\text { Ausentes }\end{array}$ & Algoritmos & Média Geral & Média Majority & KNNImpute & SKNN & IKNNImpute & KMI & EACImpute \\
\hline \multirow{7}{*}{$10 \%$} & Média Geral & $(-/-/-)$ & $(0 / 0 / 9)$ & $(0 / 0 / 9)$ & $(0 / 0 / 9)$ & $(0 / 0 / 9)$ & $(0 / 0 / 9)$ & $(0 / 0 / 9)$ \\
\hline & Média Majority & $(9 / 0 / 0)$ & $(-/-/-)$ & $(0 / 0 / 9)$ & $(0 / 0 / 9)$ & $(0 / 0 / 9)$ & $(9 / 0 / 0)$ & $(0 / 0 / 9)$ \\
\hline & KNNImpute & $(9 / 0 / 0)$ & $(9 / 0 / 0)$ & $(-/-/-)$ & $(5 / 0 / 4)$ & $(4 / 1 / 4)$ & $(9 / 0 / 0)$ & $(9 / 0 / 0)$ \\
\hline & SKNN & $(9 / 0 / 0)$ & $(9 / 0 / 0)$ & $(4 / 0 / 5)$ & $(-/-/-)$ & $(3 / 1 / 5)$ & $(9 / 0 / 0)$ & $(9 / 0 / 0)$ \\
\hline & IKNNImpute & $(9 / 0 / 0)$ & $(9 / 0 / 0)$ & $(4 / 1 / 4)$ & $(5 / 1 / 3)$ & $(-/-/-)$ & $(9 / 0 / 0)$ & $(9 / 0 / 0)$ \\
\hline & KMI & $(9 / 0 / 0)$ & $(0 / 0 / 9)$ & $(0 / 0 / 9)$ & $(0 / 0 / 9)$ & $(0 / 0 / 9)$ & $(-/-/-)$ & $(0 / 0 / 9)$ \\
\hline & EACImpute & $(9 / 0 / 0)$ & $(9 / 0 / 0)$ & $(0 / 0 / 9)$ & $(0 / 0 / 9)$ & $(0 / 0 / 9)$ & $(9 / 0 / 0)$ & $(-/-/-)$ \\
\hline \multirow{7}{*}{$30 \%$} & Média Geral & $(-/-/-)$ & $(0 / 0 / 9)$ & $(0 / 0 / 9)$ & $(0 / 0 / 9)$ & $(0 / 0 / 9)$ & $(0 / 0 / 9)$ & $(0 / 0 / 9)$ \\
\hline & Média Majority & $(9 / 0 / 0)$ & $(-/-/-)$ & $(0 / 0 / 9)$ & $(0 / 0 / 9)$ & $(0 / 0 / 9)$ & $(9 / 0 / 0)$ & $(0 / 0 / 9)$ \\
\hline & KNNImpute & $(9 / 0 / 0)$ & $(9 / 0 / 0)$ & $(-/-/-)$ & $(8 / 0 / 1)$ & $(2 / 1 / 6)$ & $(9 / 0 / 0)$ & $(9 / 0 / 0)$ \\
\hline & SKNN & $(9 / 0 / 0)$ & $(9 / 0 / 0)$ & $(1 / 0 / 8)$ & $(-/-/-)$ & $(0 / 0 / 9)$ & $(9 / 0 / 0)$ & $(9 / 0 / 0)$ \\
\hline & IKNNImpute & $(9 / 0 / 0)$ & $(9 / 0 / 0)$ & $(6 / 1 / 2)$ & $(9 / 0 / 0)$ & $(-/-/-)$ & $(9 / 0 / 0)$ & $(9 / 0 / 0)$ \\
\hline & KMI & $(9 / 0 / 0)$ & $(0 / 0 / 9)$ & $(0 / 0 / 9)$ & $(0 / 0 / 9)$ & $(0 / 0 / 9)$ & $(-/-/-)$ & $(0 / 0 / 9)$ \\
\hline & EACImpute & $(9 / 0 / 0)$ & $(9 / 0 / 0)$ & $(0 / 0 / 9)$ & $(0 / 0 / 9)$ & $(0 / 0 / 9)$ & $(9 / 0 / 0)$ & $(-/-/-)$ \\
\hline \multirow{7}{*}{$50 \%$} & Média Geral & $(-/-/-)$ & $(0 / 0 / 9)$ & $(0 / 0 / 9)$ & $(0 / 0 / 9)$ & $(0 / 0 / 9)$ & $(0 / 0 / 9)$ & $(0 / 0 / 9)$ \\
\hline & Média Majority & $(9 / 0 / 0)$ & $(-/-/-)$ & $(0 / 0 / 9)$ & $(0 / 0 / 9)$ & $(0 / 0 / 9)$ & $(9 / 0 / 0)$ & $(0 / 0 / 9)$ \\
\hline & KNNImpute & $(9 / 0 / 0)$ & $(9 / 0 / 0)$ & $(-/-/-)$ & $(8 / 0 / 1)$ & $(3 / 1 / 5)$ & $(9 / 0 / 0)$ & $(9 / 0 / 0)$ \\
\hline & SKNN & $(9 / 0 / 0)$ & $(9 / 0 / 0)$ & $(1 / 0 / 8)$ & $(-/-1-)$ & $(0 / 0 / 9)$ & $(9 / 0 / 0)$ & $(9 / 0 / 0)$ \\
\hline & IKNNImpute & $(9 / 0 / 0)$ & $(9 / 0 / 0)$ & $(5 / 1 / 3)$ & $(9 / 0 / 0)$ & $(-/-/-)$ & $(9 / 0 / 0)$ & $(9 / 0 / 0)$ \\
\hline & KMI & $(9 / 0 / 0)$ & $(0 / 0 / 9)$ & $(0 / 0 / 9)$ & $(0 / 0 / 9)$ & $(0 / 0 / 9)$ & $(-/-/-)$ & $(0 / 0 / 9)$ \\
\hline & EACImpute & $(9 / 0 / 0)$ & $(9 / 0 / 0)$ & $(0 / 0 / 9)$ & $(0 / 0 / 9)$ & $(0 / 0 / 9)$ & $(9 / 0 / 0)$ & $(-/-/-)$ \\
\hline \multirow{7}{*}{$70 \%$} & Média Geral & $(-/-/-)$ & $(0 / 0 / 9)$ & $(0 / 0 / 9)$ & $(0 / 0 / 9)$ & $(0 / 0 / 9)$ & $(0 / 0 / 9)$ & $(0 / 0 / 9)$ \\
\hline & Média Majority & $(9 / 0 / 0)$ & $(-/-/-)$ & $(0 / 0 / 9)$ & $(0 / 0 / 9)$ & $(0 / 0 / 9)$ & $(9 / 0 / 0)$ & $(0 / 0 / 9)$ \\
\hline & KNNImpute & $(9 / 0 / 0)$ & $(9 / 0 / 0)$ & $(-/-/-)$ & $(8 / 0 / 1)$ & $(3 / 0 / 6)$ & $(9 / 0 / 0)$ & $(9 / 0 / 0)$ \\
\hline & SKNN & $(9 / 0 / 0)$ & $(9 / 0 / 0)$ & $(1 / 0 / 8)$ & $(-/-/-)$ & $(0 / 0 / 9)$ & $(9 / 0 / 0)$ & $(9 / 0 / 0)$ \\
\hline & IKNNImpute & $(9 / 0 / 0)$ & $(9 / 0 / 0)$ & $(6 / 0 / 3)$ & $(9 / 0 / 0)$ & $(-/-/-)$ & $(9 / 0 / 0)$ & $(9 / 0 / 0)$ \\
\hline & KMI & $(9 / 0 / 0)$ & $(0 / 0 / 9)$ & $(0 / 0 / 9)$ & $(0 / 0 / 9)$ & $(0 / 0 / 9)$ & $(-/-/-)$ & $(0 / 0 / 9)$ \\
\hline & EACImpute & $(9 / 0 / 0)$ & $(9 / 0 / 0)$ & $(0 / 0 / 9)$ & $(0 / 0 / 9)$ & $(0 / 0 / 9)$ & $(9 / 0 / 0)$ & $(-/-/-)$ \\
\hline
\end{tabular}

Tabela A.17: Resultados da comparação pareada (Vitórias/Empates/derrotas) dos "ranks" médios em cada taxa de ausentes $-\overline{E Q_{N}}$ para a base de dados Segmentation.

\begin{tabular}{|c|c|c|c|c|}
\hline Pares de Algoritmos & $10 \%$ & $30 \%$ & $50 \%$ & $70 \%$ \\
\hline$<$ Média Geral,Média Majority $>$ & $(0 / 0 / 9)$ & $(0 / 0 / 9)$ & $(0 / 0 / 9)$ & $(0 / 0 / 9)$ \\
\hline$<$ Média Geral,KNNImpute $>$ & $(0 / 0 / 9)$ & $(0 / 0 / 9)$ & $(0 / 0 / 9)$ & $(0 / 0 / 9)$ \\
\hline$<$ Média Geral,SKNN $>$ & $(0 / 0 / 9)$ & $(0 / 0 / 9)$ & $(0 / 0 / 9)$ & $(0 / 0 / 9)$ \\
\hline$<$ Média Geral,IKNNImpute $>$ & $(0 / 0 / 9)$ & $(0 / 0 / 9)$ & $(0 / 0 / 9)$ & $(0 / 0 / 9)$ \\
\hline$<$ Média Geral,KMI $>$ & $(0 / 9 / 0)$ & $(0 / 9 / 0)$ & $(0 / 9 / 0)$ & $(0 / 9 / 0)$ \\
\hline$<$ Média Geral,EACImpute $>$ & $(0 / 0 / 9)$ & $(0 / 0 / 9)$ & $(0 / 0 / 9)$ & $(0 / 0 / 9)$ \\
\hline$<$ Média Majority,KNNImpute $>$ & $(0 / 0 / 9)$ & $(0 / 0 / 9)$ & $(0 / 0 / 9)$ & $(0 / 0 / 9)$ \\
\hline$<$ Média Majority,SKNN $>$ & $(0 / 0 / 9)$ & $(0 / 0 / 9)$ & $(0 / 0 / 9)$ & $(0 / 0 / 9)$ \\
\hline$<$ Média Majority,IKNNImpute $>$ & $(0 / 0 / 9)$ & $(0 / 0 / 9)$ & $(0 / 0 / 9)$ & $(0 / 0 / 9)$ \\
\hline$<$ Média Majority,KMI $>$ & $(1 / 8 / 0)$ & $(0 / 9 / 0)$ & $(0 / 9 / 0)$ & $(0 / 9 / 0)$ \\
\hline$<$ Média Majority,EACImpute $>$ & $(0 / 9 / 0)$ & $(0 / 9 / 0)$ & $(0 / 9 / 0)$ & $(0 / 9 / 0)$ \\
\hline$<$ KNNImpute,SKNN $>$ & $(0 / 9 / 0)$ & $(0 / 9 / 0)$ & $(0 / 9 / 0)$ & $(4 / 5 / 0)$ \\
\hline$<$ KNNImpute,IKNNImpute $>$ & $(0 / 9 / 0)$ & $(0 / 9 / 0)$ & $(0 / 9 / 0)$ & $(0 / 9 / 0)$ \\
\hline$<$ KNNImpute,KMI $>$ & $(9 / 0 / 0)$ & $(9 / 0 / 0)$ & $(9 / 0 / 0)$ & $(9 / 0 / 0)$ \\
\hline$<$ KNNImpute,EACImpute $>$ & $(9 / 0 / 0)$ & $(9 / 0 / 0)$ & $(9 / 0 / 0)$ & $(9 / 0 / 0)$ \\
\hline$<$ SKNN,IKNNImpute $>$ & $(0 / 9 / 0)$ & $(0 / 9 / 0)$ & $(0 / 9 / 0)$ & $(0 / 6 / 3)$ \\
\hline$<$ SKNN,KMI $>$ & $(9 / 0 / 0)$ & $(9 / 0 / 0)$ & $(9 / 0 / 0)$ & $(9 / 0 / 0)$ \\
\hline$<$ SKNN,EACImpute $>$ & $(9 / 0 / 0)$ & $(9 / 0 / 0)$ & $(5 / 4 / 0)$ & $(2 / 7 / 0)$ \\
\hline$<$ IKNNImpute,KMI $>$ & $(9 / 0 / 0)$ & $(9 / 0 / 0)$ & $(9 / 0 / 0)$ & $(9 / 0 / 0)$ \\
\hline$<$ IKNNImpute,EACImpute $>$ & $(9 / 0 / 0)$ & $(9 / 0 / 0)$ & $(9 / 0 / 0)$ & $(9 / 0 / 0)$ \\
\hline$<$ KMI,EACImpute $>$ & $(0 / 0 / 9)$ & $(0 / 0 / 9)$ & $(0 / 0 / 9)$ & $(0 / 0 / 9)$ \\
\hline & & & &
\end{tabular}

Tabela A.18: Resultados da comparação pareada (Vitórias/Empates/derrotas) em relação às diferenças estatísticas observadas em cada taxa de ausentes $-\overline{E Q_{N}}$ para a base de dados Segmentation. 


\begin{tabular}{|c|c|c|c|c|c|c|c|c|}
\hline $\begin{array}{c}\text { Taxa de } \\
\text { Ausentes }\end{array}$ & Algoritmos & Média Geral & Média Majority & KNNImpute & SKNN & IKNNImpute & KMI & EACImpute \\
\hline \multirow{7}{*}{$10 \%$} & Média Geral & $(-/-/-)$ & $(0 / 0 / 9)$ & $(0 / 0 / 9)$ & $(0 / 0 / 9)$ & $(0 / 0 / 9)$ & $(0 / 0 / 9)$ & $(0 / 0 / 9)$ \\
\hline & Média Majority & $(9 / 0 / 0)$ & $(-/-/-)$ & $(0 / 0 / 9)$ & $(0 / 0 / 9)$ & $(0 / 0 / 9)$ & $(0 / 0 / 9)$ & $(0 / 0 / 9)$ \\
\hline & KNNImpute & $(9 / 0 / 0)$ & $(9 / 0 / 0)$ & $(-/-/-)$ & $(0 / 0 / 9)$ & $(0 / 0 / 9)$ & $(9 / 0 / 0)$ & $(9 / 0 / 0)$ \\
\hline & SKNN & $(9 / 0 / 0)$ & $(9 / 0 / 0)$ & $(9 / 0 / 0)$ & $(-/-/-)$ & $(0 / 0 / 9)$ & $(9 / 0 / 0)$ & $(9 / 0 / 0)$ \\
\hline & IKNNImpute & $(9 / 0 / 0)$ & $(9 / 0 / 0)$ & $(9 / 0 / 0)$ & $(9 / 0 / 0)$ & $(-/-/-)$ & $(9 / 0 / 0)$ & $(9 / 0 / 0)$ \\
\hline & KMI & $(9 / 0 / 0)$ & $(9 / 0 / 0)$ & $(0 / 0 / 9)$ & $(0 / 0 / 9)$ & $(0 / 0 / 9)$ & $(-/ /-)$ & $(0 / 0 / 9)$ \\
\hline & EACImpute & $(9 / 0 / 0)$ & $(9 / 0 / 0)$ & $(0 / 0 / 9)$ & $(0 / 0 / 9)$ & $(0 / 0 / 9)$ & $(9 / 0 / 0)$ & $(-/-/-)$ \\
\hline \multirow{7}{*}{$30 \%$} & Média Geral & $(-/-/-)$ & $(0 / 0 / 9)$ & $(0 / 0 / 9)$ & $(0 / 0 / 9)$ & $(0 / 0 / 9)$ & $(0 / 0 / 9)$ & $(0 / 0 / 9)$ \\
\hline & Média Majority & $(9 / 0 / 0)$ & $(-/-/-)$ & $(0 / 0 / 9)$ & $(0 / 0 / 9)$ & $(0 / 0 / 9)$ & $(0 / 0 / 9)$ & $(0 / 0 / 9)$ \\
\hline & KNNImpute & $(9 / 0 / 0)$ & $(9 / 0 / 0)$ & $(-/-/-)$ & $(0 / 0 / 9)$ & $(0 / 0 / 9)$ & $(9 / 0 / 0)$ & $(9 / 0 / 0)$ \\
\hline & SKNN & $(9 / 0 / 0)$ & $(9 / 0 / 0)$ & $(9 / 0 / 0)$ & $(-/-/-)$ & $(0 / 0 / 9)$ & $(9 / 0 / 0)$ & $(9 / 0 / 0)$ \\
\hline & IKNNImpute & $(9 / 0 / 0)$ & $(9 / 0 / 0)$ & $(9 / 0 / 0)$ & $(9 / 0 / 0)$ & $(-/-/-)$ & $(9 / 0 / 0)$ & $(9 / 0 / 0)$ \\
\hline & KMI & $(9 / 0 / 0)$ & $(9 / 0 / 0)$ & $(0 / 0 / 9)$ & $(0 / 0 / 9)$ & $(0 / 0 / 9)$ & $(-/-/-)$ & $(0 / 0 / 9)$ \\
\hline & EACImpute & $(9 / 0 / 0)$ & $(9 / 0 / 0)$ & $(0 / 0 / 9)$ & $(0 / 0 / 9)$ & $(0 / 0 / 9)$ & $(9 / 0 / 0)$ & $(-/-/-)$ \\
\hline \multirow{7}{*}{$50 \%$} & Média Geral & $(-/-/-)$ & $(0 / 0 / 9)$ & $(0 / 0 / 9)$ & $(0 / 0 / 9)$ & $(0 / 0 / 9)$ & $(0 / 0 / 9)$ & $(0 / 0 / 9)$ \\
\hline & Média Majority & $(9 / 0 / 0)$ & $(-/-/-)$ & $(0 / 0 / 9)$ & $(0 / 0 / 9)$ & $(0 / 0 / 9)$ & $(0 / 0 / 9)$ & $(0 / 0 / 9)$ \\
\hline & KNNImpute & $(9 / 0 / 0)$ & $(9 / 0 / 0)$ & $(-/-/-)$ & $(0 / 0 / 9)$ & $(0 / 0 / 9)$ & $(9 / 0 / 0)$ & $(8 / 0 / 1)$ \\
\hline & SKNN & $(9 / 0 / 0)$ & $(9 / 0 / 0)$ & $(9 / 0 / 0)$ & $(-/-/-)$ & $(0 / 0 / 9)$ & $(9 / 0 / 0)$ & $(9 / 0 / 0)$ \\
\hline & IKNNImpute & $(9 / 0 / 0)$ & $(9 / 0 / 0)$ & $(9 / 0 / 0)$ & $(9 / 0 / 0)$ & $(-/-/-)$ & $(9 / 0 / 0)$ & $(9 / 0 / 0)$ \\
\hline & KMI & $(9 / 0 / 0)$ & $(9 / 0 / 0)$ & $(0 / 0 / 9)$ & $(0 / 0 / 9)$ & $(0 / 0 / 9)$ & $(-/-/-)$ & $(0 / 0 / 9)$ \\
\hline & EACImpute & $(9 / 0 / 0)$ & $(9 / 0 / 0)$ & $(1 / 0 / 8)$ & $(0 / 0 / 9)$ & $(0 / 0 / 9)$ & $(9 / 0 / 0)$ & $(-/-/-)$ \\
\hline \multirow{7}{*}{$70 \%$} & Média Geral & $(-/-/-)$ & $(0 / 0 / 9)$ & $(0 / 0 / 9)$ & $(0 / 0 / 9)$ & $(0 / 0 / 9)$ & $(0 / 0 / 9)$ & $(0 / 0 / 9)$ \\
\hline & Média Majority & $(9 / 0 / 0)$ & $(-/-/-)$ & $(0 / 0 / 9)$ & $(0 / 0 / 9)$ & $(0 / 0 / 9)$ & $(0 / 0 / 9)$ & $(0 / 0 / 9)$ \\
\hline & KNNImpute & $(9 / 0 / 0)$ & $(9 / 0 / 0)$ & $(-/-/-)$ & $(0 / 0 / 9)$ & $(0 / 0 / 9)$ & $(9 / 0 / 0)$ & $(8 / 0 / 1)$ \\
\hline & SKNN & $(9 / 0 / 0)$ & $(9 / 0 / 0)$ & $(9 / 0 / 0)$ & $(-/-/-)$ & $(0 / 0 / 9)$ & $(9 / 0 / 0)$ & $(9 / 0 / 0)$ \\
\hline & IKNNImpute & $(9 / 0 / 0)$ & $(9 / 0 / 0)$ & $(9 / 0 / 0)$ & $(9 / 0 / 0)$ & $(-/-/-)$ & $(9 / 0 / 0)$ & $(9 / 0 / 0)$ \\
\hline & KMI & $(9 / 0 / 0)$ & $(9 / 0 / 0)$ & $(0 / 0 / 9)$ & $(0 / 0 / 9)$ & $(0 / 0 / 9)$ & $(-/-/-)$ & $(0 / 0 / 9)$ \\
\hline & EACImpute & $(9 / 0 / 0)$ & $(9 / 0 / 0)$ & $(1 / 0 / 8)$ & $(0 / 0 / 9)$ & $(0 / 0 / 9)$ & $(9 / 0 / 0)$ & $(-/-/-)$ \\
\hline
\end{tabular}

Tabela A.19: Resultados da comparação pareada (Vitórias/Empates/derrotas) dos "ranks" médios em cada taxa de ausentes - viés na classificação para a base de dados Segmentation.

\begin{tabular}{|c|c|c|c|c|}
\hline Pares de Algoritmos & $10 \%$ & $30 \%$ & $50 \%$ & $70 \%$ \\
\hline$<$ Média Geral,Média Majority $>$ & $(0 / 8 / 1)$ & $(0 / 9 / 0)$ & $(0 / 9 / 0)$ & $(0 / 9 / 0)$ \\
\hline$<$ Média Geral,KNNImpute $>$ & $(0 / 0 / 9)$ & $(0 / 0 / 9)$ & $(0 / 0 / 9)$ & $(0 / 0 / 9)$ \\
\hline$<$ Média Geral,SKNN $>$ & $(0 / 0 / 9)$ & $(0 / 0 / 9)$ & $(0 / 0 / 9)$ & $(0 / 0 / 9)$ \\
\hline$<$ Média Geral,IKNNImpute $>$ & $(0 / 0 / 9)$ & $(0 / 0 / 9)$ & $(0 / 0 / 9)$ & $(0 / 0 / 9)$ \\
\hline$<$ Média Geral,KMI $>$ & $(0 / 0 / 9)$ & $(0 / 0 / 9)$ & $(0 / 0 / 9)$ & $(0 / 0 / 9)$ \\
\hline$<$ Média Geral,EACImpute $>$ & $(0 / 0 / 9)$ & $(0 / 0 / 9)$ & $(0 / 0 / 9)$ & $(0 / 0 / 9)$ \\
\hline$<$ Média Majority,KNNImpute $>$ & $(0 / 0 / 9)$ & $(0 / 0 / 9)$ & $(0 / 0 / 9)$ & $(0 / 0 / 9)$ \\
\hline$<$ Média Majority,SKNN $>$ & $(0 / 0 / 9)$ & $(0 / 0 / 9)$ & $(0 / 0 / 9)$ & $(0 / 0 / 9)$ \\
\hline$<$ Média Majority,IKNNImpute $>$ & $(0 / 0 / 9)$ & $(0 / 0 / 9)$ & $(0 / 0 / 9)$ & $(0 / 0 / 9)$ \\
\hline$<$ Média Majority,KMI $>$ & $(0 / 6 / 3)$ & $(0 / 9 / 0)$ & $(0 / 9 / 0)$ & $(0 / 9 / 0)$ \\
\hline$<$ Média Majority,EACImpute $>$ & $(0 / 1 / 8)$ & $(0 / 0 / 9)$ & $(0 / 0 / 9)$ & $(0 / 0 / 9)$ \\
\hline$<$ KNNImpute,SKNN $>$ & $(0 / 9 / 0)$ & $(0 / 9 / 0)$ & $(0 / 9 / 0)$ & $(0 / 9 / 0)$ \\
\hline$<$ KNNImpute,IKNNImpute $>$ & $(0 / 3 / 6)$ & $(0 / 0 / 9)$ & $(0 / 1 / 8)$ & $(0 / 2 / 7)$ \\
\hline$<$ KNNImpute,KMI $>$ & $(8 / 1 / 0)$ & $(9 / 0 / 0)$ & $(9 / 0 / 0)$ & $(9 / 0 / 0)$ \\
\hline$<$ KNNImpute,EACImpute $>$ & $(4 / 5 / 0)$ & $(2 / 7 / 0)$ & $(1 / 8 / 0)$ & $(0 / 9 / 0)$ \\
\hline$<$ SKNN,IKNNImpute $>$ & $(0 / 9 / 0)$ & $(0 / 9 / 0)$ & $(0 / 9 / 0)$ & $(0 / 9 / 0)$ \\
\hline$<$ SKNN,KMI $>$ & $(9 / 0 / 0)$ & $(9 / 0 / 0)$ & $(9 / 0 / 0)$ & $(9 / 0 / 0)$ \\
\hline$<$ SKNN,EACImpute $>$ & $(9 / 0 / 0)$ & $(9 / 0 / 0)$ & $(8 / 1 / 0)$ & $(8 / 1 / 0)$ \\
\hline$<$ IKNNImpute,KMI $>$ & $(9 / 0 / 0)$ & $(9 / 0 / 0)$ & $(9 / 0 / 0)$ & $(9 / 0 / 0)$ \\
\hline$<$ IKNNImpute,EACImpute $>$ & $(9 / 0 / 0)$ & $(9 / 0 / 0)$ & $(9 / 0 / 0)$ & $(9 / 0 / 0)$ \\
\hline$<$ KMI,EACImpute $>$ & $(0 / 9 / 0)$ & $(0 / 9 / 0)$ & $(0 / 8 / 1)$ & $(0 / 5 / 4)$ \\
\hline
\end{tabular}

Tabela A.20: Resultados da comparação pareada (Vitórias/Empates/derrotas) em relação às diferenças estatísticas observadas em cada taxa de ausentes - viés na classificação para a base de dados Segmentation. 


\section{A.2 Aleatório - MAR}

\section{A.2.1 Base de Dados "Sintética"}

\begin{tabular}{|c|c|c|c|c|c|c|c|}
\hline $\begin{array}{c}\text { Taxa de } \\
\text { Ausentes }\end{array}$ & Algoritmos & Média Majority & KNNImpute & SKNN & IKNNImpute & KMI & EACImpute \\
\hline \multirow{6}{*}{$10 \%$} & Média Majority & $(-/-/-)$ & $(0 / 0 / 1)$ & $(0 / 0 / 1)$ & $(0 / 0 / 1)$ & $(0 / 0 / 1)$ & $(0 / 0 / 1)$ \\
\hline & KNNImpute & $(1 / 0 / 0)$ & $(-/-/-)$ & $(0 / 0 / 1)$ & $(1 / 0 / 0)$ & $(0 / 0 / 1)$ & $(0 / 0 / 1)$ \\
\hline & SKNN & $(1 / 0 / 0)$ & $(1 / 0 / 0)$ & $(-/-/-)$ & $(1 / 0 / 0)$ & $(0 / 0 / 1)$ & $(0 / 0 / 1)$ \\
\hline & IKNNImpute & $(1 / 0 / 0)$ & $(0 / 0 / 1)$ & $(0 / 0 / 1)$ & $(-/-/-)$ & $(0 / 0 / 1)$ & $(0 / 0 / 1)$ \\
\hline & KMI & $(1 / 0 / 0)$ & $(1 / 0 / 0)$ & $(1 / 0 / 0)$ & $(1 / 0 / 0)$ & $(-/-/-)$ & $(1 / 0 / 0)$ \\
\hline & EACImpute & $(1 / 0 / 0)$ & $(1 / 0 / 0)$ & $(1 / 0 / 0)$ & $(1 / 0 / 0)$ & $(0 / 0 / 1)$ & $(-/-/-)$ \\
\hline \multirow{6}{*}{$30 \%$} & Média Majority & $(-/-/-)$ & $(0 / 0 / 1)$ & $(0 / 0 / 1)$ & $(0 / 0 / 1)$ & $(0 / 0 / 1)$ & $(0 / 0 / 1)$ \\
\hline & KNNImpute & $(1 / 0 / 0)$ & $(-/-/-)$ & $(0 / 1 / 0)$ & $(1 / 0 / 0)$ & $(0 / 0 / 1)$ & $(0 / 0 / 1)$ \\
\hline & SKNN & $(1 / 0 / 0)$ & $(0 / 1 / 0)$ & $(-/-/-)$ & $(1 / 0 / 0)$ & $(0 / 0 / 1)$ & $(0 / 0 / 1)$ \\
\hline & IKNNImpute & $(1 / 0 / 0)$ & $(0 / 0 / 1)$ & $(0 / 0 / 1)$ & $(-/-/-)$ & $(0 / 0 / 1)$ & $(0 / 0 / 1)$ \\
\hline & KMI & $(1 / 0 / 0)$ & $(1 / 0 / 0)$ & $(1 / 0 / 0)$ & $(1 / 0 / 0)$ & $(-/-/-)$ & $(1 / 0 / 0)$ \\
\hline & EACImpute & $(1 / 0 / 0)$ & $(1 / 0 / 0)$ & $(1 / 0 / 0)$ & $(1 / 0 / 0)$ & $(0 / 0 / 1)$ & $(-/-/-)$ \\
\hline \multirow{6}{*}{$50 \%$} & Média Majority & $(-/-/-)$ & $(0 / 0 / 1)$ & $(0 / 0 / 1)$ & $(0 / 0 / 1)$ & $(0 / 0 / 1)$ & $(0 / 0 / 1)$ \\
\hline & KNNImpute & $(1 / 0 / 0)$ & $(-/-/-)$ & $(1 / 0 / 0)$ & $(1 / 0 / 0)$ & $(0 / 0 / 1)$ & $(0 / 0 / 1)$ \\
\hline & SKNN & $(1 / 0 / 0)$ & $(0 / 0 / 1)$ & $(-/-/-)$ & $(1 / 0 / 0)$ & $(0 / 0 / 1)$ & $(0 / 0 / 1)$ \\
\hline & IKNNImpute & $(1 / 0 / 0)$ & $(0 / 0 / 1)$ & $(0 / 0 / 1)$ & $(-/-/-)$ & $(0 / 0 / 1)$ & $(0 / 0 / 1)$ \\
\hline & KMI & $(1 / 0 / 0)$ & $(1 / 0 / 0)$ & $(1 / 0 / 0)$ & $(1 / 0 / 0)$ & $(-/-/-)$ & $(1 / 0 / 0)$ \\
\hline & EACImpute & $(1 / 0 / 0)$ & $(1 / 0 / 0)$ & $(1 / 0 / 0)$ & $(1 / 0 / 0)$ & $(0 / 0 / 1)$ & $(-/-/-)$ \\
\hline \multirow{6}{*}{$70 \%$} & Média Majority & $(-/-/-)$ & $(0 / 0 / 1)$ & $(0 / 0 / 1)$ & $(0 / 0 / 1)$ & $(0 / 0 / 1)$ & $(0 / 0 / 1)$ \\
\hline & KNNImpute & $(1 / 0 / 0)$ & $(-/-/-)$ & $(0 / 0 / 1)$ & $(1 / 0 / 0)$ & $(0 / 0 / 1)$ & $(0 / 0 / 1)$ \\
\hline & SKNN & $(1 / 0 / 0)$ & $(1 / 0 / 0)$ & $(-/-/-)$ & $(1 / 0 / 0)$ & $(0 / 0 / 1)$ & $(0 / 0 / 1)$ \\
\hline & IKNNImpute & $(1 / 0 / 0)$ & $(0 / 0 / 1)$ & $(0 / 0 / 1)$ & $(-/-/-)$ & $(0 / 0 / 1)$ & $(0 / 0 / 1)$ \\
\hline & KMI & $(1 / 0 / 0)$ & $(1 / 0 / 0)$ & $(1 / 0 / 0)$ & $(1 / 0 / 0)$ & $(-/-/-)$ & $(1 / 0 / 0)$ \\
\hline & EACImpute & $(1 / 0 / 0)$ & $(1 / 0 / 0)$ & $(1 / 0 / 0)$ & $(1 / 0 / 0)$ & $(0 / 0 / 1)$ & $(-/-/-)$ \\
\hline
\end{tabular}

Tabela A.21: Resultados da comparação pareada (Vitórias/Empates/derrotas) dos "ranks" médios em cada taxa de ausentes $-\overline{E Q_{N}}$ para a base de dados Sintética.

\begin{tabular}{|c|c|c|c|c|}
\hline Pares de Algoritmos & $10 \%$ & $30 \%$ & $50 \%$ & $70 \%$ \\
\hline$<$ Média Majority,KNNImpute $>$ & $(0 / 0 / 1)$ & $(0 / 0 / 1)$ & $(0 / 0 / 1)$ & $(0 / 0 / 1)$ \\
\hline$<$ Média Majority,SKNN $>$ & $(0 / 0 / 1)$ & $(0 / 0 / 1)$ & $(0 / 0 / 1)$ & $(0 / 0 / 1)$ \\
\hline$<$ Média Majority,IKNNImpute $>$ & $(0 / 0 / 1)$ & $(0 / 1 / 0)$ & $(0 / 1 / 0)$ & $(0 / 1 / 0)$ \\
\hline$<$ Média Majority,KMI $>$ & $(0 / 0 / 1)$ & $(0 / 0 / 1)$ & $(0 / 0 / 1)$ & $(0 / 0 / 1)$ \\
\hline$<$ Média Majority,EACImpute $>$ & $(0 / 0 / 1)$ & $(0 / 0 / 1)$ & $(0 / 0 / 1)$ & $(0 / 0 / 1)$ \\
\hline$<$ KNNImpute,SKNN $>$ & $(0 / 1 / 0)$ & $(0 / 1 / 0)$ & $(0 / 1 / 0)$ & $(0 / 1 / 0)$ \\
\hline$<$ KNNImpute,IKNNImpute $>$ & $(0 / 1 / 0)$ & $(0 / 1 / 0)$ & $(1 / 0 / 0)$ & $(1 / 0 / 0)$ \\
\hline$<$ KNNImpute,KMI $>$ & $(0 / 0 / 1)$ & $(0 / 0 / 1)$ & $(0 / 0 / 1)$ & $(0 / 0 / 1)$ \\
\hline$<$ KNNImpute,EACImpute $>$ & $(0 / 0 / 1)$ & $(0 / 0 / 1)$ & $(0 / 0 / 1)$ & $(0 / 0 / 1)$ \\
\hline$<$ SKNN,IKNNImpute $>$ & $(0 / 1 / 0)$ & $(0 / 1 / 0)$ & $(1 / 0 / 0)$ & $(1 / 0 / 0)$ \\
\hline$<$ SKNN,KMI $>$ & $(0 / 0 / 1)$ & $(0 / 0 / 1)$ & $(0 / 0 / 1)$ & $(0 / 0 / 1)$ \\
\hline$<$ SKNN,EACImpute $>$ & $(0 / 0 / 1)$ & $(0 / 0 / 1)$ & $(0 / 0 / 1)$ & $(0 / 0 / 1)$ \\
\hline$<$ IKNNImpute,KMI $>$ & $(0 / 0 / 1)$ & $(0 / 0 / 1)$ & $(0 / 0 / 1)$ & $(0 / 0 / 1)$ \\
\hline$<$ IKNNImpute,EACImpute $>$ & $(0 / 0 / 1)$ & $(0 / 0 / 1)$ & $(0 / 0 / 1)$ & $(0 / 0 / 1)$ \\
\hline$<$ KMI,EACImpute $>$ & $(0 / 1 / 0)$ & $(0 / 1 / 0)$ & $(0 / 1 / 0)$ & $(0 / 1 / 0)$ \\
\hline
\end{tabular}

Tabela A.22: Resultados da comparação pareada (Vitórias/Empates/derrotas) em relação às diferenças estatísticas observadas em cada taxa de ausentes $-\overline{E Q_{N}}$ para a base de dados Sintética. 


\begin{tabular}{|c|c|c|c|c|c|c|c|}
\hline $\begin{array}{l}\text { Taxa de } \\
\text { Ausentes }\end{array}$ & Algoritmos & Média Majority & KNNImpute & SKNN & IKNNImpute & KMI & EACImpute \\
\hline \multirow{6}{*}{$10 \%$} & Média Majority & $(-/-/-)$ & $(0 / 0 / 1)$ & $(0 / 0 / 1)$ & $(0 / 0 / 1)$ & $(0 / 0 / 1)$ & $(0 / 0 / 1)$ \\
\hline & KNNImpute & $(1 / 0 / 0)$ & $(-/-/-)$ & $(0 / 1 / 0)$ & $(0 / 1 / 0)$ & $(0 / 1 / 0)$ & $(0 / 1 / 0)$ \\
\hline & SKNN & $(1 / 0 / 0)$ & $(0 / 1 / 0)$ & $(-/-/-)$ & $(0 / 1 / 0)$ & $(0 / 1 / 0)$ & $(0 / 1 / 0)$ \\
\hline & IKNNImpute & $(1 / 0 / 0)$ & $(0 / 1 / 0)$ & $(0 / 1 / 0)$ & $(-/-/-)$ & $(0 / 1 / 0)$ & $(0 / 1 / 0)$ \\
\hline & KMI & $(1 / 0 / 0)$ & $(0 / 1 / 0)$ & $(0 / 1 / 0)$ & $(0 / 1 / 0)$ & $(-/-/-)$ & $(0 / 1 / 0)$ \\
\hline & EACImpute & $(1 / 0 / 0)$ & $(0 / 1 / 0)$ & $(0 / 1 / 0)$ & $(0 / 1 / 0)$ & $(0 / 1 / 0)$ & $(-/-/-)$ \\
\hline \multirow{6}{*}{$30 \%$} & Média Majority & $(-/-/-)$ & $(0 / 0 / 1)$ & $(0 / 0 / 1)$ & $(0 / 0 / 1)$ & $(0 / 0 / 1)$ & $(0 / 0 / 1)$ \\
\hline & KNNImpute & $(1 / 0 / 0)$ & $(-/-/-)$ & $(0 / 1 / 0)$ & $(0 / 1 / 0)$ & $(0 / 1 / 0)$ & $(0 / 1 / 0)$ \\
\hline & SKNN & $(1 / 0 / 0)$ & $(0 / 1 / 0)$ & $(-/-/-)$ & $(0 / 1 / 0)$ & $(0 / 1 / 0)$ & $(0 / 1 / 0)$ \\
\hline & IKNNImpute & $(1 / 0 / 0)$ & $(0 / 1 / 0)$ & $(0 / 1 / 0)$ & $(-/-/-)$ & $(0 / 1 / 0)$ & $(0 / 1 / 0)$ \\
\hline & KMI & $(1 / 0 / 0)$ & $(0 / 1 / 0)$ & $(0 / 1 / 0)$ & $(0 / 1 / 0)$ & $(-/-/-)$ & $(0 / 1 / 0)$ \\
\hline & EACImpute & $(1 / 0 / 0)$ & $(0 / 1 / 0)$ & $(0 / 1 / 0)$ & $(0 / 1 / 0)$ & $(0 / 1 / 0)$ & $(-/-/-)$ \\
\hline \multirow{6}{*}{$50 \%$} & Média Majority & $(-/-/-)$ & $(0 / 0 / 1)$ & $(0 / 0 / 1)$ & $(0 / 0 / 1)$ & $(0 / 0 / 1)$ & $(0 / 0 / 1)$ \\
\hline & KNNImpute & $(1 / 0 / 0)$ & $(-/-/-)$ & $(0 / 1 / 0)$ & $(0 / 1 / 0)$ & $(0 / 1 / 0)$ & $(0 / 1 / 0)$ \\
\hline & SKNN & $(1 / 0 / 0)$ & $(0 / 1 / 0)$ & $(-/-/-)$ & $(0 / 1 / 0)$ & $(0 / 1 / 0)$ & $(0 / 1 / 0)$ \\
\hline & IKNNImpute & $(1 / 0 / 0)$ & $(0 / 0 / 1)$ & $(0 / 0 / 1)$ & $(-/-/-)$ & $(0 / 0 / 1)$ & $(0 / 0 / 1)$ \\
\hline & KMI & $(1 / 0 / 0)$ & $(0 / 1 / 0)$ & $(0 / 1 / 0)$ & $(0 / 1 / 0)$ & $(-/-/-)$ & $(0 / 1 / 0)$ \\
\hline & EACImpute & $(1 / 0 / 0)$ & $(0 / 1 / 0)$ & $(0 / 1 / 0)$ & $(0 / 1 / 0)$ & $(0 / 1 / 0)$ & $(-/-/-)$ \\
\hline \multirow{6}{*}{$50 \%$} & Média Majority & $(-/-/-)$ & $(0 / 0 / 1)$ & $(0 / 0 / 1)$ & $(0 / 0 / 1)$ & $(0 / 0 / 1)$ & $(0 / 0 / 1)$ \\
\hline & KNNImpute & $(1 / 0 / 0)$ & $(-/-/-)$ & $(0 / 1 / 0)$ & $(0 / 1 / 0)$ & $(0 / 1 / 0)$ & $(0 / 1 / 0)$ \\
\hline & SKNN & $(1 / 0 / 0)$ & $(0 / 1 / 0)$ & $(-/-/-)$ & $(0 / 1 / 0)$ & $(0 / 1 / 0)$ & $(0 / 1 / 0)$ \\
\hline & IKNNImpute & $(1 / 0 / 0)$ & $(0 / 0 / 1)$ & $(0 / 0 / 1)$ & $(-/-/-)$ & $(0 / 0 / 1)$ & $(0 / 0 / 1)$ \\
\hline & KMI & $(1 / 0 / 0)$ & $(0 / 1 / 0)$ & $(0 / 1 / 0)$ & $(0 / 1 / 0)$ & $(-/-/-)$ & $(0 / 1 / 0)$ \\
\hline & EACImpute & $(1 / 0 / 0)$ & $(0 / 1 / 0)$ & $(0 / 1 / 0)$ & $(0 / 1 / 0)$ & $(0 / 1 / 0)$ & $(-/-/-)$ \\
\hline
\end{tabular}

Tabela A.23: Resultados da comparação pareada (Vitórias/Empates/derrotas) dos "ranks" médios em cada taxa de ausentes - viés na classificação para a base de dados Sintética.

\begin{tabular}{|c|c|c|c|c|}
\hline Pares de Algoritmos & $10 \%$ & $30 \%$ & $50 \%$ & $70 \%$ \\
\hline$<$ Média Majority,KNNImpute $>$ & $(0 / 0 / 1)$ & $(0 / 0 / 1)$ & $(0 / 0 / 1)$ & $(0 / 0 / 1)$ \\
\hline$<$ Média Majority,SKNN $>$ & $(0 / 0 / 1)$ & $(0 / 0 / 1)$ & $(0 / 0 / 1)$ & $(0 / 0 / 1)$ \\
\hline$<$ Média Majority,IKNNImpute $>$ & $(0 / 0 / 1)$ & $(0 / 0 / 1)$ & $(0 / 0 / 1)$ & $(0 / 1 / 0)$ \\
\hline$<$ Média Majority,KMI $>$ & $(0 / 0 / 1)$ & $(0 / 0 / 1)$ & $(0 / 0 / 1)$ & $(0 / 0 / 1)$ \\
\hline$<$ Média Majority,EACImpute $>$ & $(0 / 0 / 1)$ & $(0 / 0 / 1)$ & $(0 / 0 / 1)$ & $(0 / 0 / 1)$ \\
\hline$<$ KNNImpute,SKNN $>$ & $(0 / 1 / 0)$ & $(0 / 1 / 0)$ & $(0 / 1 / 0)$ & $(0 / 1 / 0)$ \\
\hline$<$ KNNImpute,IKNNImpute $>$ & $(0 / 1 / 0)$ & $(0 / 1 / 0)$ & $(0 / 1 / 0)$ & $(1 / 0 / 0)$ \\
\hline$<$ KNNImpute,KMI $>$ & $(0 / 1 / 0)$ & $(0 / 1 / 0)$ & $(0 / 1 / 0)$ & $(0 / 1 / 0)$ \\
\hline$<$ KNNImpute,EACImpute $>$ & $(0 / 1 / 0)$ & $(0 / 1 / 0)$ & $(0 / 1 / 0)$ & $(0 / 1 / 0)$ \\
\hline$<$ SKNN,IKNNImpute $>$ & $(0 / 1 / 0)$ & $(0 / 1 / 0)$ & $(0 / 1 / 0)$ & $(1 / 0 / 0)$ \\
\hline$<$ SKNN,KMI $>$ & $(0 / 1 / 0)$ & $(0 / 1 / 0)$ & $(0 / 1 / 0)$ & $(0 / 1 / 0)$ \\
\hline$<$ SKNN,EACImpute $>$ & $(0 / 1 / 0)$ & $(0 / 1 / 0)$ & $(0 / 1 / 0)$ & $(0 / 1 / 0)$ \\
\hline$<$ IKNNImpute,KMI $>$ & $(0 / 1 / 0)$ & $(0 / 1 / 0)$ & $(0 / 1 / 0)$ & $(0 / 0 / 1)$ \\
\hline$<$ IKNNImpute,EACImpute $>$ & $(0 / 1 / 0)$ & $(0 / 1 / 0)$ & $(0 / 1 / 0)$ & $(0 / 0 / 1)$ \\
\hline$<$ KMI,EACImpute $>$ & $(0 / 1 / 0)$ & $(0 / 1 / 0)$ & $(0 / 1 / 0)$ & $(0 / 1 / 0)$ \\
\hline
\end{tabular}

Tabela A.24: Resultados da comparação pareada (Vitórias/Empates/derrotas) em relação às diferenças estatísticas observadas em cada taxa de ausentes - viés na classificação para a base de dados Sintética. 


\section{A.2.2 Base de Dados "Íris"}

\begin{tabular}{|c|c|c|c|c|c|c|c|}
\hline $\begin{array}{c}\text { Taxa de } \\
\text { Ausentes }\end{array}$ & Algoritmos & Média Majority & KNNImpute & SKNN & IKNNImpute & KMI & EACImpute \\
\hline \multirow{6}{*}{$10 \%$} & Média Majority & $(-/-/-)$ & $(0 / 0 / 1)$ & $(0 / 0 / 1)$ & $(0 / 0 / 1)$ & $(0 / 0 / 1)$ & $(0 / 0 / 1)$ \\
\hline & KNNImpute & $(1 / 0 / 0)$ & $(-/-/-)$ & $(0 / 0 / 1)$ & $(1 / 0 / 0)$ & $(0 / 0 / 1)$ & $(0 / 0 / 1)$ \\
\hline & SKNN & $(1 / 0 / 0)$ & $(1 / 0 / 0)$ & $(-/-/-)$ & $(1 / 0 / 0)$ & $(0 / 0 / 1)$ & $(0 / 0 / 1)$ \\
\hline & IKNNImpute & $(1 / 0 / 0)$ & $(0 / 0 / 1)$ & $(0 / 0 / 1)$ & $(-/-/-)$ & $(0 / 0 / 1)$ & $(0 / 0 / 1)$ \\
\hline & KMI & $(1 / 0 / 0)$ & $(1 / 0 / 0)$ & $(1 / 0 / 0)$ & $(1 / 0 / 0)$ & $(-/-/-)$ & $(1 / 0 / 0)$ \\
\hline & EACImpute & $(1 / 0 / 0)$ & $(1 / 0 / 0)$ & $(1 / 0 / 0)$ & $(1 / 0 / 0)$ & $(0 / 0 / 1)$ & $(-/-/-)$ \\
\hline \multirow{6}{*}{$30 \%$} & Média Majority & $(-/-/-)$ & $(0 / 0 / 1)$ & $(0 / 0 / 1)$ & $(0 / 0 / 1)$ & $(0 / 0 / 1)$ & $(0 / 0 / 1)$ \\
\hline & KNNImpute & $(1 / 0 / 0)$ & $(-/-/-)$ & $(0 / 1 / 0)$ & $(1 / 0 / 0)$ & $(0 / 0 / 1)$ & $(0 / 0 / 1)$ \\
\hline & SKNN & $(1 / 0 / 0)$ & $(0 / 1 / 0)$ & $(-/-/-)$ & $(1 / 0 / 0)$ & $(0 / 0 / 1)$ & $(0 / 0 / 1)$ \\
\hline & IKNNImpute & $(1 / 0 / 0)$ & $(0 / 0 / 1)$ & $(0 / 0 / 1)$ & $(-/-/-)$ & $(0 / 0 / 1)$ & $(0 / 0 / 1)$ \\
\hline & KMI & $(1 / 0 / 0)$ & $(1 / 0 / 0)$ & $(1 / 0 / 0)$ & $(1 / 0 / 0)$ & $(-/-/-)$ & $(1 / 0 / 0)$ \\
\hline & EACImpute & $(1 / 0 / 0)$ & $(1 / 0 / 0)$ & $(1 / 0 / 0)$ & $(1 / 0 / 0)$ & $(0 / 0 / 1)$ & $(-/-/-)$ \\
\hline \multirow{6}{*}{$50 \%$} & Média Majority & $(-/-/-)$ & $(0 / 0 / 1)$ & $(0 / 0 / 1)$ & $(0 / 0 / 1)$ & $(0 / 0 / 1)$ & $(0 / 0 / 1)$ \\
\hline & KNNImpute & $(1 / 0 / 0)$ & $(-/-/-)$ & $(1 / 0 / 0)$ & $(1 / 0 / 0)$ & $(0 / 0 / 1)$ & $(0 / 0 / 1)$ \\
\hline & SKNN & $(1 / 0 / 0)$ & $(0 / 0 / 1)$ & $(-/-/-)$ & $(1 / 0 / 0)$ & $(0 / 0 / 1)$ & $(0 / 0 / 1)$ \\
\hline & IKNNImpute & $(1 / 0 / 0)$ & $(0 / 0 / 1)$ & $(0 / 0 / 1)$ & $(-/-/-)$ & $(0 / 0 / 1)$ & $(0 / 0 / 1)$ \\
\hline & KMI & $(1 / 0 / 0)$ & $(1 / 0 / 0)$ & $(1 / 0 / 0)$ & $(1 / 0 / 0)$ & $(-/-/-)$ & $(1 / 0 / 0)$ \\
\hline & EACImpute & $(1 / 0 / 0)$ & $(1 / 0 / 0)$ & $(1 / 0 / 0)$ & $(1 / 0 / 0)$ & $(0 / 0 / 1)$ & $(-/-/-)$ \\
\hline \multirow{6}{*}{$70 \%$} & Média Majority & $(-/-/-)$ & $(0 / 0 / 1)$ & $(0 / 0 / 1)$ & $(0 / 0 / 1)$ & $(0 / 0 / 1)$ & $(0 / 0 / 1)$ \\
\hline & KNNImpute & $(1 / 0 / 0)$ & $(-/-/-)$ & $(0 / 0 / 1)$ & $(1 / 0 / 0)$ & $(0 / 0 / 1)$ & $(0 / 0 / 1)$ \\
\hline & SKNN & $(1 / 0 / 0)$ & $(1 / 0 / 0)$ & $(-/-/-)$ & $(1 / 0 / 0)$ & $(0 / 0 / 1)$ & $(0 / 0 / 1)$ \\
\hline & IKNNImpute & $(1 / 0 / 0)$ & $(0 / 0 / 1)$ & $(0 / 0 / 1)$ & $(-/-/-)$ & $(0 / 0 / 1)$ & $(0 / 0 / 1)$ \\
\hline & KMI & $(1 / 0 / 0)$ & $(1 / 0 / 0)$ & $(1 / 0 / 0)$ & $(1 / 0 / 0)$ & $(-/-/-)$ & $(1 / 0 / 0)$ \\
\hline & EACImpute & $(1 / 0 / 0)$ & $(1 / 0 / 0)$ & $(1 / 0 / 0)$ & $(1 / 0 / 0)$ & $(0 / 0 / 1)$ & $(-/-/-)$ \\
\hline
\end{tabular}

Tabela A.25: Resultados da comparação pareada (Vitórias/Empates/derrotas) dos "ranks" médios em cada taxa de ausentes $-\overline{E Q_{N}}$ para a base de dados Íris-1.

\begin{tabular}{|c|c|c|c|c|}
\hline Pares de Algoritmos & $10 \%$ & $30 \%$ & $50 \%$ & $70 \%$ \\
\hline$<$ Média Majority,KNNImpute $>$ & $(0 / 0 / 1)$ & $(0 / 0 / 1)$ & $(0 / 0 / 1)$ & $(0 / 0 / 1)$ \\
\hline$<$ Média Majority,SKNN $>$ & $(0 / 0 / 1)$ & $(0 / 0 / 1)$ & $(0 / 0 / 1)$ & $(0 / 0 / 1)$ \\
\hline$<$ Média Majority,IKNNImpute $>$ & $(0 / 0 / 1)$ & $(0 / 0 / 1)$ & $(0 / 0 / 1)$ & $(0 / 0 / 1)$ \\
\hline$<$ Média Majority,KMI $>$ & $(0 / 1 / 0)$ & $(0 / 1 / 0)$ & $(0 / 1 / 0)$ & $(0 / 1 / 0)$ \\
\hline$<$ Média Majority,EACImpute $>$ & $(0 / 1 / 0)$ & $(0 / 1 / 0)$ & $(0 / 0 / 1)$ & $(0 / 0 / 1)$ \\
\hline$<$ KNNImpute,SKNN $>$ & $(0 / 1 / 0)$ & $(0 / 1 / 0)$ & $(0 / 1 / 0)$ & $(0 / 1 / 0)$ \\
\hline$<$ KNNImpute,IKNNImpute $>$ & $(0 / 1 / 0)$ & $(0 / 1 / 0)$ & $(0 / 1 / 0)$ & $(0 / 1 / 0)$ \\
\hline$<$ KNNImpute,KMI $>$ & $(1 / 0 / 0)$ & $(1 / 0 / 0)$ & $(1 / 0 / 0)$ & $(1 / 0 / 0)$ \\
\hline$<$ KNNImpute,EACImpute $>$ & $(0 / 1 / 0)$ & $(0 / 1 / 0)$ & $(0 / 1 / 0)$ & $(0 / 1 / 0)$ \\
\hline$<$ SKNN,IKNNImpute $>$ & $(0 / 1 / 0)$ & $(0 / 1 / 0)$ & $(0 / 1 / 0)$ & $(0 / 1 / 0)$ \\
\hline$<$ SKNN,KMI $>$ & $(1 / 0 / 0)$ & $(1 / 0 / 0)$ & $(1 / 0 / 0)$ & $(1 / 0 / 0)$ \\
\hline$<$ SKNN,EACImpute $>$ & $(0 / 1 / 0)$ & $(0 / 1 / 0)$ & $(1 / 0 / 0)$ & $(1 / 0 / 0)$ \\
\hline$<$ IKNNImpute,KMI $>$ & $(1 / 0 / 0)$ & $(1 / 0 / 0)$ & $(1 / 0 / 0)$ & $(1 / 0 / 0)$ \\
\hline$<$ IKNNImpute,EACImpute $>$ & $(0 / 1 / 0)$ & $(0 / 1 / 0)$ & $(0 / 1 / 0)$ & $(0 / 1 / 0)$ \\
\hline$<$ KMI,EACImpute $>$ & $(0 / 1 / 0)$ & $(0 / 1 / 0)$ & $(0 / 0 / 1)$ & $(0 / 0 / 1)$ \\
\hline
\end{tabular}

Tabela A.26: Resultados da comparação pareada (Vitórias/Empates/derrotas) em relação às diferenças estatísticas observadas em cada taxa de ausentes $-\overline{E Q_{N}}$ para a base de dados Íris1. 


\begin{tabular}{|c|c|c|c|c|c|c|c|}
\hline $\begin{array}{c}\text { Taxa de } \\
\text { Ausentes }\end{array}$ & Algoritmos & Média Majority & KNNImpute & SKNN & IKNNImpute & KMI & EACImpute \\
\hline \multirow{6}{*}{$10 \%$} & Média Majority & $(-/-/-)$ & $(0 / 0 / 1)$ & $(0 / 0 / 1)$ & $(0 / 0 / 1)$ & $(1 / 0 / 0)$ & $(0 / 0 / 1)$ \\
\hline & KNNImpute & $(1 / 0 / 0)$ & $(-/-/-)$ & $(1 / 0 / 0)$ & $(0 / 0 / 1)$ & $(1 / 0 / 0)$ & $(1 / 0 / 0)$ \\
\hline & SKNN & $(1 / 0 / 0)$ & $(0 / 0 / 1)$ & $(-/-/-)$ & $(0 / 0 / 1)$ & $(1 / 0 / 0)$ & $(1 / 0 / 0)$ \\
\hline & IKNNImpute & $(1 / 0 / 0)$ & $(1 / 0 / 0)$ & $(1 / 0 / 0)$ & $(-/-/-)$ & $(1 / 0 / 0)$ & $(1 / 0 / 0)$ \\
\hline & KMI & $(0 / 0 / 1)$ & $(0 / 0 / 1)$ & $(0 / 0 / 1)$ & $(0 / 0 / 1)$ & $(-/-/-)$ & $(0 / 0 / 1)$ \\
\hline & EACImpute & $(1 / 0 / 0)$ & $(0 / 0 / 1)$ & $(0 / 0 / 1)$ & $(0 / 0 / 1)$ & $(1 / 0 / 0)$ & $(-/-/-)$ \\
\hline \multirow{6}{*}{$30 \%$} & Média Majority & $(-/-/-)$ & $(0 / 0 / 1)$ & $(0 / 0 / 1)$ & $(0 / 0 / 1)$ & $(0 / 0 / 1)$ & $(0 / 0 / 1)$ \\
\hline & KNNImpute & $(1 / 0 / 0)$ & $(-/-/-)$ & $(0 / 0 / 1)$ & $(1 / 0 / 0)$ & $(1 / 0 / 0)$ & $(1 / 0 / 0)$ \\
\hline & SKNN & $(1 / 0 / 0)$ & $(1 / 0 / 0)$ & $(-/-/-)$ & $(1 / 0 / 0)$ & $(1 / 0 / 0)$ & $(1 / 0 / 0)$ \\
\hline & IKNNImpute & $(1 / 0 / 0)$ & $(0 / 0 / 1)$ & $(0 / 0 / 1)$ & $(-/-/-)$ & $(1 / 0 / 0)$ & $(1 / 0 / 0)$ \\
\hline & KMI & $(1 / 0 / 0)$ & $(0 / 0 / 1)$ & $(0 / 0 / 1)$ & $(0 / 0 / 1)$ & $(-/-/-)$ & $(0 / 0 / 1)$ \\
\hline & EACImpute & $(1 / 0 / 0)$ & $(0 / 0 / 1)$ & $(0 / 0 / 1)$ & $(0 / 0 / 1)$ & $(1 / 0 / 0)$ & $(-/-/-)$ \\
\hline \multirow{6}{*}{$50 \%$} & Média Majority & $(-/-/-)$ & $(0 / 0 / 1)$ & $(0 / 0 / 1)$ & $(0 / 0 / 1)$ & $(1 / 0 / 0)$ & $(0 / 0 / 1)$ \\
\hline & KNNImpute & $(1 / 0 / 0)$ & $(-/-/-)$ & $(0 / 0 / 1)$ & $(1 / 0 / 0)$ & $(1 / 0 / 0)$ & $(1 / 0 / 0)$ \\
\hline & SKNN & $(1 / 0 / 0)$ & $(1 / 0 / 0)$ & $(-/-/-)$ & $(1 / 0 / 0)$ & $(1 / 0 / 0)$ & $(1 / 0 / 0)$ \\
\hline & IKNNImpute & $(1 / 0 / 0)$ & $(0 / 0 / 1)$ & $(0 / 0 / 1)$ & $(-/-/-)$ & $(1 / 0 / 0)$ & $(1 / 0 / 0)$ \\
\hline & KMI & $(0 / 0 / 1)$ & $(0 / 0 / 1)$ & $(0 / 0 / 1)$ & $(0 / 0 / 1)$ & $(-/-/-)$ & $(0 / 0 / 1)$ \\
\hline & EACImpute & $(1 / 0 / 0)$ & $(0 / 0 / 1)$ & $(0 / 0 / 1)$ & $(0 / 0 / 1)$ & $(1 / 0 / 0)$ & $(-/-/-)$ \\
\hline \multirow{6}{*}{$70 \%$} & Média Majority & $(-/-/-)$ & $(0 / 0 / 1)$ & $(0 / 0 / 1)$ & $(0 / 0 / 1)$ & $(0 / 0 / 1)$ & $(0 / 0 / 1)$ \\
\hline & KNNImpute & $(1 / 0 / 0)$ & $(-/-/-)$ & $(0 / 0 / 1)$ & $(1 / 0 / 0)$ & $(1 / 0 / 0)$ & $(1 / 0 / 0)$ \\
\hline & SKNN & $(1 / 0 / 0)$ & $(1 / 0 / 0)$ & $(-/-/-)$ & $(1 / 0 / 0)$ & $(1 / 0 / 0)$ & $(1 / 0 / 0)$ \\
\hline & IKNNImpute & $(1 / 0 / 0)$ & $(0 / 0 / 1)$ & $(0 / 0 / 1)$ & $(-/-/-)$ & $(1 / 0 / 0)$ & $(0 / 0 / 1)$ \\
\hline & KMI & $(1 / 0 / 0)$ & $(0 / 0 / 1)$ & $(0 / 0 / 1)$ & $(0 / 0 / 1)$ & $(-/-/-)$ & $(0 / 0 / 1)$ \\
\hline & EACImpute & $(1 / 0 / 0)$ & $(0 / 0 / 1)$ & $(0 / 0 / 1)$ & $(1 / 0 / 0)$ & $(1 / 0 / 0)$ & $(-/-/-)$ \\
\hline
\end{tabular}

Tabela A.27: Resultados da comparação pareada (Vitórias/Empates/derrotas) dos "ranks" médios em cada taxa de ausentes $-\overline{E Q_{N}}$ para a base de dados Íris-2.

\begin{tabular}{|c|c|c|c|c|}
\hline Pares de Algoritmos & $10 \%$ & $30 \%$ & $50 \%$ & $70 \%$ \\
\hline$<$ Média Majority,KNNImpute $>$ & $(0 / 0 / 1)$ & $(0 / 0 / 1)$ & $(0 / 0 / 1)$ & $(0 / 0 / 1)$ \\
\hline$<$ Média Majority,SKNN $>$ & $(0 / 0 / 1)$ & $(0 / 0 / 1)$ & $(0 / 0 / 1)$ & $(0 / 0 / 1)$ \\
\hline$<$ Média Majority,IKNNImpute $>$ & $(0 / 0 / 1)$ & $(0 / 0 / 1)$ & $(0 / 0 / 1)$ & $(0 / 0 / 1)$ \\
\hline$<$ Média Majority,KMI $>$ & $(0 / 1 / 0)$ & $(0 / 0 / 1)$ & $(0 / 1 / 0)$ & $(0 / 0 / 1)$ \\
\hline$<$ Média Majority,EACImpute $>$ & $(0 / 0 / 1)$ & $(0 / 0 / 1)$ & $(0 / 0 / 1)$ & $(0 / 0 / 1)$ \\
\hline$<$ KNNImpute,SKNN $>$ & $(0 / 1 / 0)$ & $(0 / 1 / 0)$ & $(0 / 1 / 0)$ & $(0 / 1 / 0)$ \\
\hline$<$ KNNImpute,IKNNImpute $>$ & $(0 / 1 / 0)$ & $(0 / 1 / 0)$ & $(0 / 1 / 0)$ & $(0 / 1 / 0)$ \\
\hline$<$ KNNImpute,KMI $>$ & $(1 / 0 / 0)$ & $(0 / 1 / 0)$ & $(1 / 0 / 0)$ & $(0 / 1 / 0)$ \\
\hline$<$ KNNImpute,EACImpute $>$ & $(0 / 1 / 0)$ & $(0 / 1 / 0)$ & $(0 / 1 / 0)$ & $(0 / 1 / 0)$ \\
\hline$<$ SKNN,IKNNImpute $>$ & $(0 / 1 / 0)$ & $(0 / 1 / 0)$ & $(0 / 1 / 0)$ & $(0 / 1 / 0)$ \\
\hline$<$ SKNN,KMI $>$ & $(1 / 0 / 0)$ & $(1 / 0 / 0)$ & $(1 / 0 / 0)$ & $(0 / 1 / 0)$ \\
\hline$<$ SKNN,EACImpute $>$ & $(0 / 1 / 0)$ & $(0 / 1 / 0)$ & $(0 / 1 / 0)$ & $(0 / 1 / 0)$ \\
\hline$<$ IKNNImpute,KMI $>$ & $(1 / 0 / 0)$ & $(1 / 0 / 0)$ & $(1 / 0 / 0)$ & $(1 / 0 / 0)$ \\
\hline$<$ IKNNImpute,EACImpute $>$ & $(0 / 1 / 0)$ & $(1 / 0 / 0)$ & $(0 / 1 / 0)$ & $(0 / 1 / 0)$ \\
\hline$<$ KMI,EACImpute $>$ & $(0 / 1 / 0)$ & $(0 / 1 / 0)$ & $(0 / 0 / 1)$ & $(0 / 1 / 0)$ \\
\hline
\end{tabular}

Tabela A.28: Resultados da comparação pareada (Vitórias/Empates/derrotas) em relação às diferenças estatísticas observadas em cada taxa de ausentes $-\overline{E Q_{N}}$ para a base de dados Íris2. 


\begin{tabular}{|c|c|c|c|c|c|c|c|}
\hline $\begin{array}{c}\text { Taxa de } \\
\text { Ausentes }\end{array}$ & Algoritmos & Média Majority & KNNImpute & SKNN & IKNNImpute & KMI & EACImpute \\
\hline \multirow{6}{*}{$10 \%$} & Média Majority & $(-/-/-)$ & $(0 / 0 / 1)$ & $(0 / 0 / 1)$ & $(0 / 0 / 1)$ & $(1 / 0 / 0)$ & $(0 / 0 / 1)$ \\
\hline & KNNImpute & $(1 / 0 / 0)$ & $(-/-/-)$ & $(1 / 0 / 0)$ & $(0 / 0 / 1)$ & $(1 / 0 / 0)$ & $(1 / 0 / 0)$ \\
\hline & SKNN & $(1 / 0 / 0)$ & $(0 / 0 / 1)$ & $(-/-/-)$ & $(0 / 0 / 1)$ & $(1 / 0 / 0)$ & $(1 / 0 / 0)$ \\
\hline & IKNNImpute & $(1 / 0 / 0)$ & $(1 / 0 / 0)$ & $(1 / 0 / 0)$ & $(-/-/-)$ & $(1 / 0 / 0)$ & $(1 / 0 / 0)$ \\
\hline & KMI & $(0 / 0 / 1)$ & $(0 / 0 / 1)$ & $(0 / 0 / 1)$ & $(0 / 0 / 1)$ & $(-/-/-)$ & $(0 / 0 / 1)$ \\
\hline & EACImpute & $(1 / 0 / 0)$ & $(0 / 0 / 1)$ & $(0 / 0 / 1)$ & $(0 / 0 / 1)$ & $(1 / 0 / 0)$ & $(-/-/-)$ \\
\hline \multirow{6}{*}{$30 \%$} & Média Majority & $(-/-/-)$ & $(0 / 0 / 1)$ & $(0 / 0 / 1)$ & $(0 / 0 / 1)$ & $(0 / 0 / 1)$ & $(0 / 0 / 1)$ \\
\hline & KNNImpute & $(1 / 0 / 0)$ & $(-/-/-)$ & $(0 / 0 / 1)$ & $(1 / 0 / 0)$ & $(1 / 0 / 0)$ & $(1 / 0 / 0)$ \\
\hline & SKNN & $(1 / 0 / 0)$ & $(1 / 0 / 0)$ & $(-/-/-)$ & $(1 / 0 / 0)$ & $(1 / 0 / 0)$ & $(1 / 0 / 0)$ \\
\hline & IKNNImpute & $(1 / 0 / 0)$ & $(0 / 0 / 1)$ & $(0 / 0 / 1)$ & $(-/-/-)$ & $(1 / 0 / 0)$ & $(1 / 0 / 0)$ \\
\hline & KMI & $(1 / 0 / 0)$ & $(0 / 0 / 1)$ & $(0 / 0 / 1)$ & $(0 / 0 / 1)$ & $(-/-/-)$ & $(0 / 0 / 1)$ \\
\hline & EACImpute & $(1 / 0 / 0)$ & $(0 / 0 / 1)$ & $(0 / 0 / 1)$ & $(0 / 0 / 1)$ & $(1 / 0 / 0)$ & $(-/-/-)$ \\
\hline \multirow{6}{*}{$50 \%$} & Média Majority & $(-/-/-)$ & $(0 / 0 / 1)$ & $(0 / 0 / 1)$ & $(0 / 0 / 1)$ & $(1 / 0 / 0)$ & $(0 / 0 / 1)$ \\
\hline & KNNImpute & $(1 / 0 / 0)$ & $(-/-/-)$ & $(0 / 0 / 1)$ & $(1 / 0 / 0)$ & $(1 / 0 / 0)$ & $(1 / 0 / 0)$ \\
\hline & SKNN & $(1 / 0 / 0)$ & $(1 / 0 / 0)$ & $(-/-/-)$ & $(1 / 0 / 0)$ & $(1 / 0 / 0)$ & $(1 / 0 / 0)$ \\
\hline & IKNNImpute & $(1 / 0 / 0)$ & $(0 / 0 / 1)$ & $(0 / 0 / 1)$ & $(-/-/-)$ & $(1 / 0 / 0)$ & $(1 / 0 / 0)$ \\
\hline & KMI & $(0 / 0 / 1)$ & $(0 / 0 / 1)$ & $(0 / 0 / 1)$ & $(0 / 0 / 1)$ & $(-/-/-)$ & $(0 / 0 / 1)$ \\
\hline & EACImpute & $(1 / 0 / 0)$ & $(0 / 0 / 1)$ & $(0 / 0 / 1)$ & $(0 / 0 / 1)$ & $(1 / 0 / 0)$ & $(-/-/-)$ \\
\hline \multirow{6}{*}{$70 \%$} & Média Majority & $(-/-/-)$ & $(0 / 0 / 1)$ & $(0 / 0 / 1)$ & $(0 / 0 / 1)$ & $(0 / 0 / 1)$ & $(0 / 0 / 1)$ \\
\hline & KNNImpute & $(1 / 0 / 0)$ & $(-/-/-)$ & $(0 / 0 / 1)$ & $(1 / 0 / 0)$ & $(1 / 0 / 0)$ & $(1 / 0 / 0)$ \\
\hline & SKNN & $(1 / 0 / 0)$ & $(1 / 0 / 0)$ & $(-/-/-)$ & $(1 / 0 / 0)$ & $(1 / 0 / 0)$ & $(1 / 0 / 0)$ \\
\hline & IKNNImpute & $(1 / 0 / 0)$ & $(0 / 0 / 1)$ & $(0 / 0 / 1)$ & $(-/-/-)$ & $(1 / 0 / 0)$ & $(0 / 0 / 1)$ \\
\hline & KMI & $(1 / 0 / 0)$ & $(0 / 0 / 1)$ & $(0 / 0 / 1)$ & $(0 / 0 / 1)$ & $(-/-/-)$ & $(0 / 0 / 1)$ \\
\hline & EACImpute & $(1 / 0 / 0)$ & $(0 / 0 / 1)$ & $(0 / 0 / 1)$ & $(1 / 0 / 0)$ & $(1 / 0 / 0)$ & $(-/-/-)$ \\
\hline
\end{tabular}

Tabela A.29: Resultados da comparação pareada (Vitórias/Empates/derrotas) dos "ranks" médios em cada taxa de ausentes $-\overline{E Q_{N}}$ para a base de dados Íris-3.

\begin{tabular}{|c|c|c|c|c|}
\hline Pares de Algoritmos & $10 \%$ & $30 \%$ & $50 \%$ & $70 \%$ \\
\hline <Média Majority,KNNImpute $>$ & $(0 / 0 / 1)$ & $(0 / 0 / 1)$ & $(0 / 0 / 1)$ & $(0 / 0 / 1)$ \\
\hline$<$ Média Majority,SKNN $>$ & $(0 / 0 / 1)$ & $(0 / 0 / 1)$ & $(0 / 0 / 1)$ & $(0 / 0 / 1)$ \\
\hline <Média Majority,IKNNImpute $>$ & $(0 / 0 / 1)$ & $(0 / 0 / 1)$ & $(0 / 0 / 1)$ & $(0 / 0 / 1)$ \\
\hline$<$ Média Majority,KMI $>$ & $(0 / 1 / 0)$ & $(0 / 1 / 0)$ & $(0 / 1 / 0)$ & $(0 / 1 / 0)$ \\
\hline$<$ Média Majority,EACImpute $>$ & $(0 / 1 / 0)$ & $(0 / 0 / 1)$ & $(0 / 1 / 0)$ & $(0 / 0 / 1)$ \\
\hline$<$ KNNImpute,SKNN $>$ & $(0 / 1 / 0)$ & $(0 / 1 / 0)$ & $(0 / 1 / 0)$ & $(0 / 1 / 0)$ \\
\hline$<$ KNNImpute,IKNNImpute $>$ & $(0 / 1 / 0)$ & $(0 / 1 / 0)$ & $(0 / 1 / 0)$ & $(0 / 1 / 0)$ \\
\hline$<$ KNNImpute,KMI $>$ & $(1 / 0 / 0)$ & $(1 / 0 / 0)$ & $(1 / 0 / 0)$ & $(1 / 0 / 0)$ \\
\hline$<$ KNNImpute,EACImpute $>$ & $(0 / 1 / 0)$ & $(1 / 0 / 0)$ & $(1 / 0 / 0)$ & $(0 / 1 / 0)$ \\
\hline$<$ SKNN,IKNNImpute $>$ & $(0 / 1 / 0)$ & $(0 / 1 / 0)$ & $(0 / 1 / 0)$ & $(0 / 1 / 0)$ \\
\hline$<$ SKNN,KMI $>$ & $(1 / 0 / 0)$ & $(1 / 0 / 0)$ & $(1 / 0 / 0)$ & $(1 / 0 / 0)$ \\
\hline$<$ SKNN,EACImpute $>$ & $(0 / 1 / 0)$ & $(1 / 0 / 0)$ & $(1 / 0 / 0)$ & $(0 / 1 / 0)$ \\
\hline$<$ IKNNImpute,KMI $>$ & $(1 / 0 / 0)$ & $(1 / 0 / 0)$ & $(1 / 0 / 0)$ & $(1 / 0 / 0)$ \\
\hline$<$ IKNNImpute,EACImpute $>$ & $(1 / 0 / 0)$ & $(0 / 1 / 0)$ & $(1 / 0 / 0)$ & $(0 / 1 / 0)$ \\
\hline$<$ KMI,EACImpute $>$ & $(0 / 0 / 1)$ & $(0 / 0 / 1)$ & $(0 / 0 / 1)$ & $(0 / 0 / 1)$ \\
\hline
\end{tabular}

Tabela A.30: Resultados da comparação pareada (Vitórias/Empates/derrotas) em relação as diferenças estatísticas observadas em cada taxa de ausentes $-\overline{E Q_{N}}$ para a base de dados Íris3. 


\section{A.2.3 Base de Dados "Segmentation"}

\begin{tabular}{|c|c|c|c|c|c|c|c|}
\hline $\begin{array}{c}\text { Taxa de } \\
\text { Ausentes }\end{array}$ & Algoritmos & Média Majority & KNNImpute & SKNN & IKNNImpute & KMI & EACImpute \\
\hline \multirow{6}{*}{$10 \%$} & Média Majority & $(-/-/-)$ & $(0 / 0 / 1)$ & $(0 / 0 / 1)$ & $(0 / 0 / 1)$ & $(0 / 0 / 1)$ & $(0 / 0 / 1)$ \\
\hline & KNNImpute & $(1 / 0 / 0)$ & $(-/-/-)$ & $(0 / 0 / 1)$ & $(1 / 0 / 0)$ & $(1 / 0 / 0)$ & $(1 / 0 / 0)$ \\
\hline & SKNN & $(1 / 0 / 0)$ & $(1 / 0 / 0)$ & $(-/-/-)$ & $(1 / 0 / 0)$ & $(1 / 0 / 0)$ & $(1 / 0 / 0)$ \\
\hline & IKNNImpute & $(1 / 0 / 0)$ & $(0 / 0 / 1)$ & $(0 / 0 / 1)$ & $(-/-/-)$ & $(1 / 0 / 0)$ & $(1 / 0 / 0)$ \\
\hline & KMI & $(1 / 0 / 0)$ & $(0 / 0 / 1)$ & $(0 / 0 / 1)$ & $(0 / 0 / 1)$ & $(-/-/-)$ & $(0 / 0 / 1)$ \\
\hline & EACImpute & $(1 / 0 / 0)$ & $(0 / 0 / 1)$ & $(0 / 0 / 1)$ & $(0 / 0 / 1)$ & $(1 / 0 / 0)$ & $(-/-/-)$ \\
\hline \multirow{6}{*}{$30 \%$} & Média Majority & $(-/-/-)$ & $(0 / 0 / 1)$ & $(0 / 0 / 1)$ & $(0 / 0 / 1)$ & $(1 / 0 / 0)$ & $(0 / 0 / 1)$ \\
\hline & KNNImpute & $(1 / 0 / 0)$ & $(-/-/-)$ & $(0 / 0 / 1)$ & $(1 / 0 / 0)$ & $(1 / 0 / 0)$ & $(1 / 0 / 0)$ \\
\hline & SKNN & $(1 / 0 / 0)$ & $(1 / 0 / 0)$ & $(-/-/-)$ & $(1 / 0 / 0)$ & $(1 / 0 / 0)$ & $(1 / 0 / 0)$ \\
\hline & IKNNImpute & $(1 / 0 / 0)$ & $(0 / 0 / 1)$ & $(0 / 0 / 1)$ & $(-/-/-)$ & $(1 / 0 / 0)$ & $(0 / 0 / 1)$ \\
\hline & KMI & $(0 / 0 / 1)$ & $(0 / 0 / 1)$ & $(0 / 0 / 1)$ & $(0 / 0 / 1)$ & $(-/-/-)$ & $(0 / 0 / 1)$ \\
\hline & EACImpute & $(1 / 0 / 0)$ & $(0 / 0 / 1)$ & $(0 / 0 / 1)$ & $(1 / 0 / 0)$ & $(1 / 0 / 0)$ & $(-/-/-)$ \\
\hline \multirow{6}{*}{$50 \%$} & Média Majority & $(-/-/-)$ & $(0 / 0 / 1)$ & $(0 / 0 / 1)$ & $(0 / 0 / 1)$ & $(0 / 0 / 1)$ & $(0 / 0 / 1)$ \\
\hline & KNNImpute & $(1 / 0 / 0)$ & $(-/-/-)$ & $(0 / 0 / 1)$ & $(1 / 0 / 0)$ & $(1 / 0 / 0)$ & $(0 / 0 / 1)$ \\
\hline & SKNN & $(1 / 0 / 0)$ & $(1 / 0 / 0)$ & $(-/-/-)$ & $(1 / 0 / 0)$ & $(1 / 0 / 0)$ & $(1 / 0 / 0)$ \\
\hline & IKNNImpute & $(1 / 0 / 0)$ & $(0 / 0 / 1)$ & $(0 / 0 / 1)$ & $(-/-/-)$ & $(1 / 0 / 0)$ & $(0 / 0 / 1)$ \\
\hline & KMI & $(1 / 0 / 0)$ & $(0 / 0 / 1)$ & $(0 / 0 / 1)$ & $(0 / 0 / 1)$ & $(-/-/-)$ & $(0 / 0 / 1)$ \\
\hline & EACImpute & $(1 / 0 / 0)$ & $(1 / 0 / 0)$ & $(0 / 0 / 1)$ & $(1 / 0 / 0)$ & $(1 / 0 / 0)$ & $(-/-/-)$ \\
\hline \multirow{6}{*}{$70 \%$} & Média Majority & $(-/-/-)$ & $(0 / 0 / 1)$ & $(0 / 0 / 1)$ & $(0 / 0 / 1)$ & $(0 / 0 / 1)$ & $(0 / 0 / 1)$ \\
\hline & KNNImpute & $(1 / 0 / 0)$ & $(-/-/-)$ & $(0 / 0 / 1)$ & $(1 / 0 / 0)$ & $(1 / 0 / 0)$ & $(0 / 0 / 1)$ \\
\hline & SKNN & $(1 / 0 / 0)$ & $(1 / 0 / 0)$ & $(-/-/-)$ & $(1 / 0 / 0)$ & $(1 / 0 / 0)$ & $(0 / 0 / 1)$ \\
\hline & IKNNImpute & $(1 / 0 / 0)$ & $(0 / 0 / 1)$ & $(0 / 0 / 1)$ & $(-/-/-)$ & $(1 / 0 / 0)$ & $(0 / 0 / 1)$ \\
\hline & KMI & $(1 / 0 / 0)$ & $(0 / 0 / 1)$ & $(0 / 0 / 1)$ & $(0 / 0 / 1)$ & $(-/-/-)$ & $(0 / 0 / 1)$ \\
\hline & EACImpute & $(1 / 0 / 0)$ & $(1 / 0 / 0)$ & $(1 / 0 / 0)$ & $(1 / 0 / 0)$ & $(1 / 0 / 0)$ & $(-/-/-)$ \\
\hline
\end{tabular}

Tabela A.31: Resultados da comparação pareada (Vitórias/Empates/derrotas) dos "ranks" médios em cada taxa de ausentes $-\overline{E Q_{N}}$ para a base de dados Segmentation.

\begin{tabular}{|c|c|c|c|c|}
\hline Pares de Algoritmos & $10 \%$ & $30 \%$ & $50 \%$ & $70 \%$ \\
\hline$<$ Média Majority,KNNImpute $>$ & $(0 / 0 / 1)$ & $(0 / 0 / 1)$ & $(0 / 0 / 1)$ & $(0 / 0 / 1)$ \\
\hline$<$ Média Majority,SKNN $>$ & $(0 / 0 / 1)$ & $(0 / 0 / 1)$ & $(0 / 0 / 1)$ & $(0 / 0 / 1)$ \\
\hline$<$ Média Majority,IKNNImpute $>$ & $(0 / 0 / 1)$ & $(0 / 0 / 1)$ & $(0 / 0 / 1)$ & $(0 / 0 / 1)$ \\
\hline$<$ Média Majority,KMI $>$ & $(0 / 1 / 0)$ & $(0 / 1 / 0)$ & $(0 / 1 / 0)$ & $(0 / 1 / 0)$ \\
\hline$<$ Média Majority,EACImpute $>$ & $(0 / 0 / 1)$ & $(0 / 0 / 1)$ & $(0 / 0 / 1)$ & $(0 / 0 / 1)$ \\
\hline$<$ KNNImpute,SKNN $>$ & $(0 / 1 / 0)$ & $(0 / 1 / 0)$ & $(0 / 1 / 0)$ & $(0 / 1 / 0)$ \\
\hline$<$ KNNImpute,IKNNImpute $>$ & $(0 / 1 / 0)$ & $(0 / 1 / 0)$ & $(1 / 0 / 0)$ & $(0 / 1 / 0)$ \\
\hline$<$ KNNImpute,KMI $>$ & $(1 / 0 / 0)$ & $(1 / 0 / 0)$ & $(1 / 0 / 0)$ & $(1 / 0 / 0)$ \\
\hline$<$ KNNImpute,EACImpute $>$ & $(0 / 1 / 0)$ & $(0 / 1 / 0)$ & $(0 / 1 / 0)$ & $(0 / 1 / 0)$ \\
\hline$<$ SKNN,IKNNImpute $>$ & $(0 / 1 / 0)$ & $(1 / 0 / 0)$ & $(1 / 0 / 0)$ & $(1 / 0 / 0)$ \\
\hline$<$ SKNN,KMI $>$ & $(1 / 0 / 0)$ & $(1 / 0 / 0)$ & $(1 / 0 / 0)$ & $(1 / 0 / 0)$ \\
\hline$<$ SKNN,EACImpute $>$ & $(0 / 1 / 0)$ & $(0 / 1 / 0)$ & $(0 / 1 / 0)$ & $(0 / 1 / 0)$ \\
\hline$<$ IKNNImpute,KMI $>$ & $(1 / 0 / 0)$ & $(1 / 0 / 0)$ & $(0 / 1 / 0)$ & $(0 / 1 / 0)$ \\
\hline$<$ IKNNImpute,EACImpute $>$ & $(0 / 1 / 0)$ & $(0 / 1 / 0)$ & $(0 / 0 / 1)$ & $(0 / 0 / 1)$ \\
\hline$<$ KMI,EACImpute $>$ & $(0 / 0 / 1)$ & $(0 / 0 / 1)$ & $(0 / 0 / 1)$ & $(0 / 0 / 1)$ \\
\hline
\end{tabular}

Tabela A.32: Resultados da comparação pareada (Vitórias/Empates/derrotas) em relação às diferenças estatísticas em cada taxa de ausentes $-\overline{E Q_{N}}$ para a base de dados Segmentation. 


\begin{tabular}{|c|c|c|c|c|}
\hline Pares de Algoritmos & $10 \%$ & $30 \%$ & $50 \%$ & $70 \%$ \\
\hline$<$ Média Majority,KNNImpute $>$ & $(0 / 0 / 1)$ & $(0 / 0 / 1)$ & $(0 / 0 / 1)$ & $(0 / 0 / 1)$ \\
\hline$<$ Média Majority,SKNN $>$ & $(0 / 0 / 1)$ & $(0 / 0 / 1)$ & $(0 / 0 / 1)$ & $(0 / 0 / 1)$ \\
\hline$<$ Média Majority,IKNNImpute $>$ & $(0 / 0 / 1)$ & $(0 / 0 / 1)$ & $(0 / 0 / 1)$ & $(0 / 0 / 1)$ \\
\hline$<$ Média Majority,KMI $>$ & $(0 / 0 / 1)$ & $(0 / 1 / 0)$ & $(0 / 0 / 1)$ & $(0 / 0 / 1)$ \\
\hline$<$ Média Majority,EACImpute $>$ & $(0 / 0 / 1)$ & $(0 / 0 / 1)$ & $(0 / 0 / 1)$ & $(0 / 0 / 1)$ \\
\hline$<$ KNNImpute,SKNN $>$ & $(0 / 1 / 0)$ & $(0 / 1 / 0)$ & $(0 / 1 / 0)$ & $(0 / 1 / 0)$ \\
\hline$<$ KNNImpute,IKNNImpute $>$ & $(0 / 1 / 0)$ & $(0 / 1 / 0)$ & $(0 / 1 / 0)$ & $(1 / 0 / 0)$ \\
\hline$<$ KNNImpute,KMI $>$ & $(0 / 1 / 0)$ & $(1 / 0 / 0)$ & $(1 / 0 / 0)$ & $(0 / 1 / 0)$ \\
\hline$<$ KNNImpute,EACImpute $>$ & $(0 / 1 / 0)$ & $(0 / 1 / 0)$ & $(0 / 1 / 0)$ & $(0 / 1 / 0)$ \\
\hline$<$ SKNN,IKNNImpute $>$ & $(0 / 1 / 0)$ & $(0 / 1 / 0)$ & $(0 / 1 / 0)$ & $(1 / 0 / 0)$ \\
\hline$<$ SKNN,KMI $>$ & $(0 / 1 / 0)$ & $(1 / 0 / 0)$ & $(1 / 0 / 0)$ & $(0 / 1 / 0)$ \\
\hline$<$ SKNN,EACImpute $>$ & $(0 / 1 / 0)$ & $(0 / 1 / 0)$ & $(0 / 1 / 0)$ & $(0 / 1 / 0)$ \\
\hline$<$ IKNNImpute,KMI $>$ & $(0 / 1 / 0)$ & $(1 / 0 / 0)$ & $(0 / 1 / 0)$ & $(0 / 1 / 0)$ \\
\hline$<$ IKNNImpute,EACImpute $>$ & $(0 / 1 / 0)$ & $(0 / 1 / 0)$ & $(0 / 1 / 0)$ & $(0 / 0 / 1)$ \\
\hline$<$ KMI,EACImpute $>$ & $(0 / 1 / 0)$ & $(0 / 0 / 1)$ & $(0 / 0 / 1)$ & $(0 / 1 / 0)$ \\
\hline
\end{tabular}

Tabela A.33: Resultados da comparação pareada (Vitórias/Empates/derrotas) em relação às diferenças estatísticas observadas em cada taxa de ausentes - viés na classificação para a base de dados Segmentation. 